\title{
The development of molecularly imprinted polymers for sensor and colorimetric assay applications
}

Citation for published version (APA):

Lowdon, J. (2021). The development of molecularly imprinted polymers for sensor and colorimetric assay applications. [Doctoral Thesis, Maastricht University]. Maastricht University. https://doi.org/10.26481/dis.20211130jl

Document status and date:

Published: 01/01/2021

DOI:

10.26481/dis.20211130jl

Document Version:

Publisher's PDF, also known as Version of record

\section{Please check the document version of this publication:}

- A submitted manuscript is the version of the article upon submission and before peer-review. There can be important differences between the submitted version and the official published version of record.

People interested in the research are advised to contact the author for the final version of the publication, or visit the DOI to the publisher's website.

- The final author version and the galley proof are versions of the publication after peer review.

- The final published version features the final layout of the paper including the volume, issue and page numbers.

Link to publication

\footnotetext{
General rights rights.

- You may freely distribute the URL identifying the publication in the public portal. please follow below link for the End User Agreement:

www.umlib.nl/taverne-license

Take down policy

If you believe that this document breaches copyright please contact us at:

repository@maastrichtuniversity.nl

providing details and we will investigate your claim.
}

Copyright and moral rights for the publications made accessible in the public portal are retained by the authors and/or other copyright owners and it is a condition of accessing publications that users recognise and abide by the legal requirements associated with these

- Users may download and print one copy of any publication from the public portal for the purpose of private study or research.

- You may not further distribute the material or use it for any profit-making activity or commercial gain

If the publication is distributed under the terms of Article $25 \mathrm{fa}$ of the Dutch Copyright Act, indicated by the "Taverne" license above, 


\section{The Development of Molecularly Imprinted \\ Polymers for Sensor and Colorimetric Assay Applications}




\section{The Development of Molecularly Imprinted Polymers for Sensor and Colorimetric Assay Applications}

\section{THESIS}

Ter verkrijging van de graad van doctor aan de Universiteit Maastricht, op gezag van de Rector Magnificus, Prof. Dr. Rianne M. Letschert volgens het besluit van het College van Decanen, in het openbaar te verdedigen op

November $30^{\text {th }} 2021$

By

Joseph W. Lowdon

Born May 19th 1994, Crewe, United Kingdom 


\section{Promotor}

Prof. Dr. Thomas J. Cleij

Dr. Bart van Grinsven

\section{Copromotor}

Dr. Hanne Diliën

\section{Beoordelingscommissie}

Chair:

Prof. Dr. Maarten Honing

\section{Juryleden:}

Dr. Katrien Bernaerts

Prof. Dr. Sven Ingebrandt

Dr. Laura Kollau

Prof. Dr. Michael Schöning

Prof. Dr. Dirk Vanderzande 


\section{Contents}

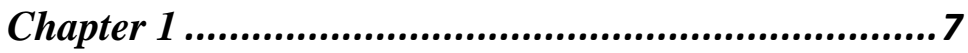

Sensing the issue (what's the problem?) ...................... 8

Introduction to chemical sensing ............................... 8

Biological receptors.............................................. 10

Molecularly Imprinted Polymers................................ 15

Readout platforms ................................................ 20

Quartz crystal microbalance (QCM) ............................ 20

Electrochemical impedance ............................................... 21

Thermal readout techniques ........................................... 21

Optical platforms ............................................................ 23

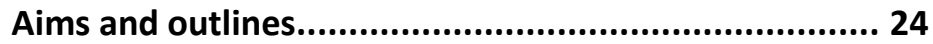

Chapter 2 ............................................................ 35

Review paper preface:......................................... 36

Review paper: MIPs for Commercial Application in Lowcost Sensors and Assays - an Overview of the Current Status Quo ............................................................. 37

Chapter 3 ......................................................8 87

Surface grafted polymers preface: .............................. 88

Research paper: Surface Grafted Molecularly Imprinted Polymeric receptor layers for Thermal Detection of the New Psychoactive Substance 2-methoxphenidine ........ 89

Chapter 4 .............................................. 117

Substrate displacement colorimetry (SDC) preface: .....118 
Research paper: Substrate Displacement Colorimetry for the Detection of Diarylethylamines.

Chapter 5

MIP based dye displacement assay for amphetamine preface:

Research paper: A Molecularly Imprinted Polymer Based Dye Displacement Assay for the Rapid Visual Detection of Amphetamine in Urine

Chapter 6

Creating a dye displacement assay for the detection of antibiotics preface:

Research paper: Colorimetric Sensing of Amoxicillin

Facilitated by Molecularly Imprinted Polymers.

Chapter 7

General Discussion 219

Appendices

Summary .230

Valorisation .232

Curriculum Vitae 236

Publications list 237

Acknowledgements 239 


\section{Chapter 1}

General introduction 


\section{Sensing the issue (what's the problem?)}

As a species we currently hold the capability to analyze any compound that we desire. Different forms of molecular analysis can be applied to yield specific information based upon the properties we wish to observe and report. This ability to harness technology in such a way has shaped the way we tackle disease, pollution, climate change, and even the human condition. Though an aspect that has been overlooked is the increasing complexity and subsequent cost that enables us to develop and utilize these methodologies. This intellectual and financial freshold is the limiting factor that prevents these analytical instruments reaching key groups that could benefit from them the most. The question that then remains is; How do we progress further and expand past the current state-of-the-art technology while tackling these issues?

The following thesis aims to provide a remedy to this question, highlighting the field of polymeric receptors known as molecularly imprinted polymers as a potential solution. To fully understand how this technology is currently placed, a brief history of chemical sensing is explored before specifically focusing upon molecularly imprinted polymers and their current applications.

\section{Introduction to chemical sensing}

A chemical sensor is defined as a self-contained device that is capable of reporting analytical data in real time regarding a specified chemical species present in its proximity [1]. A chemical sensor must therefore execute two simultaneous functions, the ability to recognize and to transduce. The recognition element of the sensor must therefore interact with a desired analyte in a unique manner, yielding selectivity [2]. The transducer thereafter has the task of converting the presence of an analyte interacting with the receptor as some form of tangible signal (Figure 1) [3].

The ability to selectively detect and quantify molecular species is an advancement that has propelled modern day medicine, forensics, analytical science and chemistry as a whole [4-7]. It is a field of scientific research that is wide reaching, affecting everyone in some capacity. Small developments in this area have the potential to cause long term and pronounced changes to our everyday lives. 


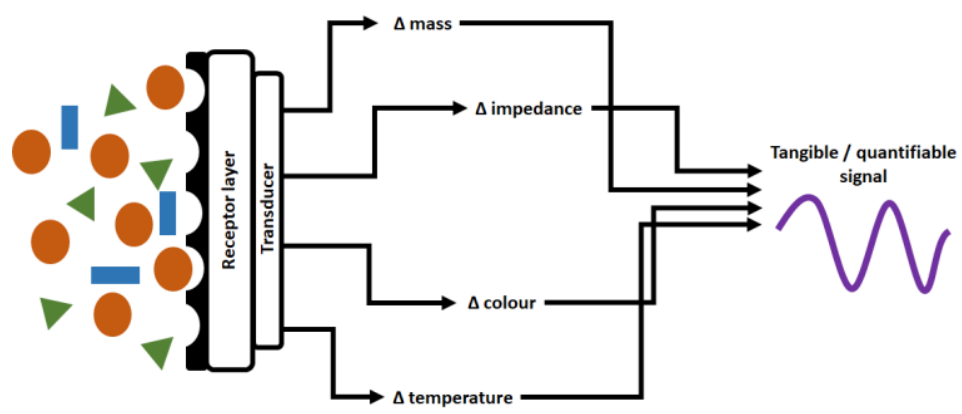

Figure 1. An artistic intereptation of the principles behind a simple chemical sensor, demonstrating how an event occurring at the receptor layer is then transuced into a tangible signal.

The field of chemical sensing has arguably always existed in some form, though the methods that we now utilize are more sophisticated and advanced than any that have come before. Haber and Klemensiewicz, in the early 1900's, are responsible for the inception of the first modern chemical sensing device as per definition [8]. They introduced the concept of detecting ions with electric charge, enabling ion recognition with numerous reagents and materials that have an opposing electrical charge to the analyte in question [9-13]. Refinement to the sensing material allowed the selectivity of the device to be tailored based on ionic receptor size and analyte peculiarity, thus the first commercially available form of the ion sensor was introduced in 1936 together with the Beckman pH meter [14]. This device exemplifies the use of a receptor in conjunction with a transducer extremely well, yet it is only the tip of the metaphorical iceberg of chemical sensing.

Electrostatic attraction is just one of the affinity interactions that can be exploited in the realms of chemical sensing. Other noncovalent binding interactions such as Van der Waals interactions and hydrogen bonding can be used to reversibly bind molecular species, forming complexes with a molecular species depending the chemical functionalities present [15-17]. Ultimately, the binding interaction is selected reflecting the chemical properties of the desired analyte, matching hydrogen bond donors with hydrogen bond acceptors, positive charge with negative charge, and maximizing van der Waals interactions [18]. Molecular recognition is not only dependent upon the pairing of affinity interactions, it is also derived from the orientation of these 
interactions relative to the molecular species in question [19]. Conformation therefore plays a vital role in the development and selection of a recognition element in chemical sensing devices [20-22].

\section{Biological receptors}

Synthesizing recognition elements that incorporate the aforementioned features is not an easy feat, though inspiration for such materials is easily drawn from nature [23-25]. Enzymes and antibodies have been using the aforementioned molecular recognition mechanism for centuries, catalyzing biological processes and protecting biological systems from foreign pathogenic invaders [26-28]. Millennia of evolution have therefore generated the ultimate molecular machines, evolved specifically for the purpose of binding a target substrate [29]. The direct implementation of antibodies and enzymes was therefore inevitable, with the glucose enzyme electrode (invented by Leland C Clark, Jr) being the first of its kind in the 1960's [30]. The technology coupled a thin layer of glucose oxidase (GOx) on an oxygen electrode (Clark electrode), thus enabling an indirect methodology of determining the amount of glucose present by monitoring the amount of oxygen consumed during the enzymatic reaction (Figure 2).

This breakthrough was further elaborated on throughout the coming decades, introducing a moving needle meter in the 1970's that could indicate blood glucose levels within a minute, before the eventually being marketed towards home use in the early 1980's [31].

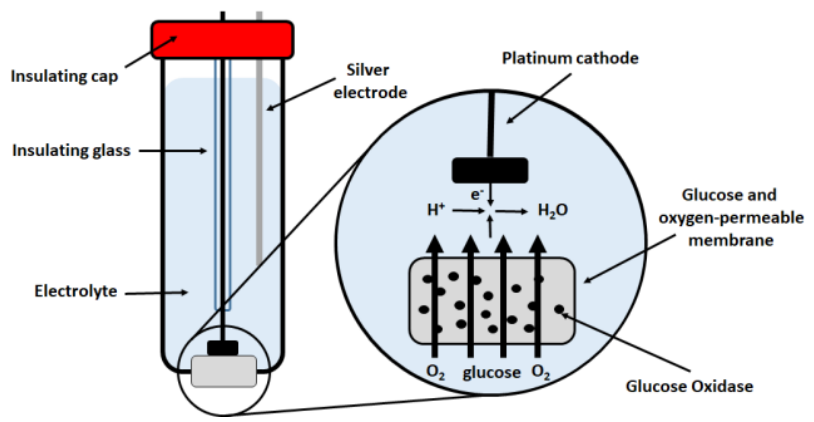

Figure 2. Schematic diagram of a modified Clark electrode, showing how glucose oxidase can be immobolised into the oxygen permeable membrane and therefore reducing the amount of oxygen reach the inner electrode and giving an indirect quantification of the amount of glucose present. 
In the same period, non-meter test strips were introduced to the market, offering the rapid sensing of the presence of glucose by means of a simple paper strip that could change colour. Similar to the glucose meter the paperbased test strips utilize GOx, though extra components such as horseradish peroxidase (HRP) and 2,2'-azino-bis(3-ethylbenzthiazoline-6-sulphonic acid) (ABTS) are required for the colorimetric reaction [32]. In essence, the GOx converts glucose into D-glucono-1,5-lactone and hydrogen peroxide, which then interacts with HRP and can be seen as a colorimetric change by the presence of the ABTS. Though extremely rapid and effective, it is argued that the methodology lacks precision as no readout is coupled with the technology. This said, the lightweight and portable nature of the format is of inherent value, meaning that the assay format can be utilized in many scenarios where other equipment would not be able to operate.

Lateral flow tests (LFT) extended the capacity in which enzymes and antibodies can be used to detect the presence of an analyte. Their initial inception was in the 1940's being derived from paper chromatography, though instead incorporated varying chemical and biological reagents across a horizontal substrate that a liquid sample could easily pass over and interact with (Figure 3) [33]. Their main appeal being complex reactions can be undertaken without the need for specialized or costly equipment, on small sample sizes. The home pregnancy test is a prime example of a LFT that has seen huge success, comprising of a simple plastic body that contains channels with both mobile and immobilized antibodies. 

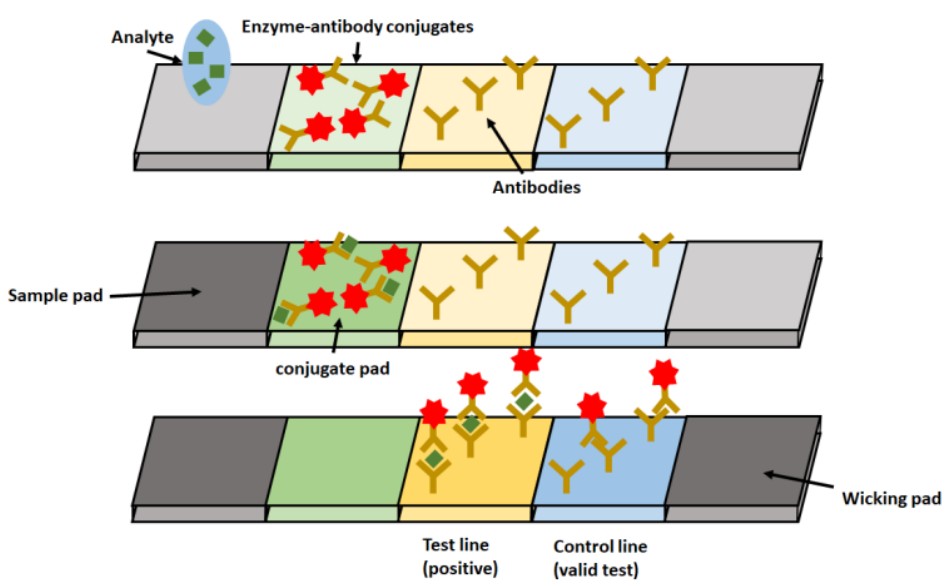

Figure 3. Generic scheme for a lateral flow test (LFT) demonstrating how the sample containing the analyte is introduced to the sample pad, how the analyte interacts with the enzyme-antibody conjugates, and how a positive test is confirmed by the binding of the analyte to the immobilized antibodies.

Upon providing a urine sample on the sample pad, the urine flows down a channel containing enzyme-modified antibodies specific for the hormone hCG. The modified antibodies bind to the hormone before moving further down the assay towards immobilized antibodies in the test zone. The immobilized antibodies in the test zone bind the hCG further, with the enzyme-linked antibodies already bound to the hCG produces a colorimetric response that can be observed on the test line of the device [34]. This is just one of many such devices designed towards the detection of hormones, small molecules, and other disease markers. The assay can be modified by using different enzymeantibody conjugates to introduce a host of quantifiable physical properties (e.g. fluorophores, chromophores, radioisotopes etc.) [35].

Enzyme-linked immunosorbent assays (ELISA) are another variation of biological component based sensors, and operate on a similar fundamental level to LFT's. The assay formats primarily focuses on the detection of proteins by use of solid-phase modified-antibodies than can ligate the target analyte. Enzymes are then incubated with the resulting bioconjugates to induce a quantifiable colour change (Figure 4) [36]. This format of assay is a prominent diagnostic tool in the fields of medicine, biotechnology, and industrial quality 
control due to its more complex nature [37]. Multiple variations of ELISA have been developed (direct, sandwich, competitive, reverse), providing different analytical approaches depending on the desired analyte and the analytical matrix in question [38-40].

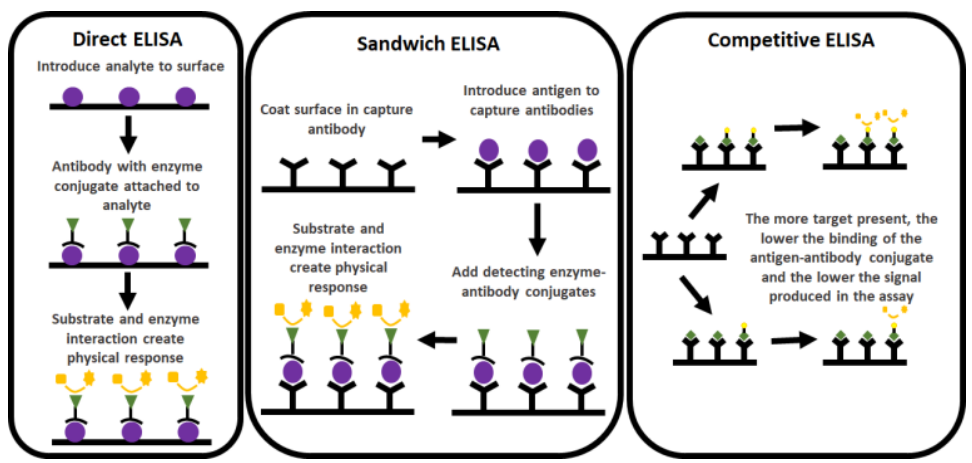

Figure 4. Variations of the ELISA (e.g. direct, sandwich and competitive) process for detecting antigens in aqueous medium.

Besides enzymes and antibodies, in recent years there has been an increase in the number of biosensors incorporating DNA as a recognition element [41]. This development has stemed directly from our greater understanding of genomes, and the corresponding tidalwave of raw genetic information there is to analyze. DNA based biosensors therefore consist of an immobilized probe DNA that is capable of detecting complimentary DNA sequences by DNADNA hybridization (figure 5) [42]. Classical versions of this methodology exist in the form of gel electrophoresis and southern blotting, though when compared with more modern sensing methods these prove time extensive. It is therefore common place to immobilize the probe DNA on a transducer that is capable of converting the binding event at the surface into a tangible signal [43]. One such example of this is Campbel et al. who bound DNA to a polymer coated piezoelectric crystal, enabling the direct detection of binding events between the DNA and target species by a Quartz Crystal Microbalance (QCM) [44]. As the probe DNA interacts with the target species the mass on the crystal increases, facilitating the change in mass to be registered as a change in the oscilating frequency of QCM. Other examples include the use of thermal readout techniques, chromophores, fluorophores, and nanoparticles bound to 
the probe DNA that can be utilized when trying to detect the binding of a target [45-47].

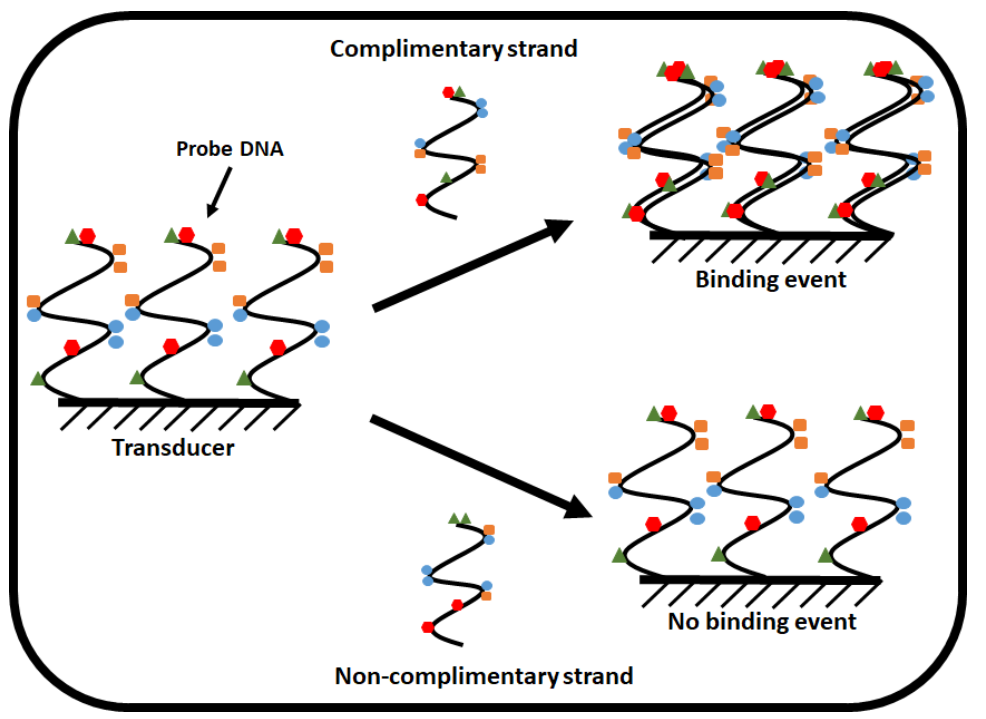

Figure 5. An artistic interpretation of the mechanism behind DNA based biosensors, demonstrating how the probe DNA would interact with complimentary and noncomplimentary DNA.

Unfortunately, the benefits of evolution only extend towards the biological systems and environments that facilitated the iteration of these biological receptors in the first place. The use of biological recognition elements is therefore preferable under physiological conditions (in general $37^{\circ} \mathrm{C}$ and mild $\mathrm{pH}$ conditions), with conditions outside these tolerances impeding the ability of the biological receptors to function due to denaturation [48, 49]. Positioning the current state of research firmly at the point of designing bio-inspired receptor materials that function on the same level as their biological counterparts, yet do not suffer the same pit falls due to harsh physical environments [50]. One promising bio-inspired technology is the field of molecularly imprinted polymers. 


\section{Molecularly Imprinted Polymers}

Molecularly Imprinted Polymers (MIPs) are highly crosslinked synthetic polymeric receptors containing specialised nanocavities complimentary to a given analyte [51]. These receptors are akin to their biological counterparts, though do not suffer the effects of denaturation in harsh physical environments [52]. The crosslinked nature of the polymeric matrix preserves the conformational structure of the generated receptor sites within the material, guaranteeing nanocavity integrity and performance [53]. This increased resilience allows MIPs to be applied to situations that would normally be impractical, opening a whole host of analytical scenarios where MIPs can be benefitted from [54-58].

The inception of MIPs occurred in the early $20^{\text {th }}$ century, with research being conducted on silica pore structures and how their "activation" (polymerization) with sulphuric acid yielded a positive correlation between the amount of porous material present and the loading capacities observed [59]. Further investigation demonstrated that a pattern molecule could be added to the synthetic process, inadvertently conducting the first molecular imprinting experiment for the extraction of dye molecules [60]. The components and terms used in the synthesis of MIPs over the course of the following decades matured with the technology, with a functional monomer, functional crosslinker, porogen, template molecule and initiator molecule being the new standard constituent building blocks of a MIP [61-63]. Each component plays a critical role, with each component bringing a key facet to the synthesis that is imperative for the success of the receptor.

The functional monomer provides affinity interactions with the template, offering ionic, hydrogen bonding, and/or Van der Waals interactions [64]. It is therefore logical that the monomer selected has complimentary functionalities to the target analyte, matching acidic with basic functionalities and visa-versa. Pi-stacking functionalities are also commonplace within the realms of monomer selection; though tend to be used in a complimentary manner alongside strong ionic/hydrogen-bonding interactions. Functional monomers tend to be small molecules that can easily orientate themselves around the template molecule, optimizing potential interactions and therefore generate nanocavities with higher levels of molecular interaction (Figure 6) [65, 66]. Another critical feature of the functional monomer is the presence of a single double bond, thus enabling the integration into a polymeric network under polymerization conditions by means of strong covalent bonding [67]. 


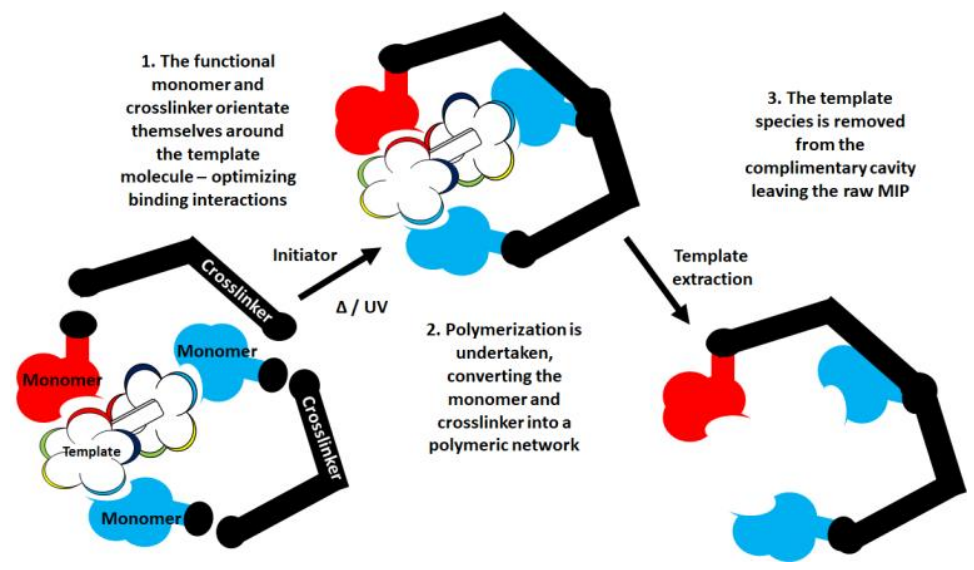

Figure 6. An artistic interpretation of the general self-assembly mechanism for the formation of the receptor site in the molecularly imprinted polymeric network, and the extraction of the template molecule leaving a vacant nanocavity.

The functional crosslinker on the other hand focuses less on the functionalities present, and is more reliant on numerous double bonds that facilitate the crosslinking (bridging) of the synthesized polymeric chains [68]. It is common place to select crosslinkers with 2 to 3 double bonds, with the greater number of doubles forming more rigid polymers [69]. The crosslinkers can also contain chemical functionalities (amides and esters) that are capable of interacting with the template molecule, though the interactions are limited due to the bulker/higher molecular weight nature of the crosslinking agents [70].

Orientation of the functional monomer/crosslinker around the template molecule can be achieved by simply combining these components in a pot, though issues arise when components are in different physical states at specific conditions. To aid this process a porogen is used. In essence, a porogen is a non-protic solvent that allows pore formation while dispersing all aforementioned components so they can interact optimally. The porogen selected should not interact in any meaningful way with the template molecule or other components, with its only tasking being to dissolve components and to help form pours within the formed polymeric network [71, 72].

To help facilitate the formation of the polymeric network by free radical polymerization the presence of an initiator molecule is often required. This 
component is responsible for the initiation of the polymerization process and is dependent on multiple factors [73]. The main consideration made when selecting the initiator molecule is the conditons that will be used for activation (thermochemical /photochemical). These conditions are dependent on the template molecule being used, with certain species degrading under thermal or photochemical conditions [74,75]. The most widely used initiators produce free radical species when exposed to these conditions, with examples being azo containing compounds and/or peroxides for the production of MIPs [76, 77].

The most common reagents for the aforementioned components include methacrylic acid/acrylamide/acrylic acid (monomer), ethylene glycol dimethacrylate (EGDMA)/trimethylolpropane trimethacrylate (TMPTM) /divinyl benzene (DVB) (crosslinker), DMSO (porogen) and Azobisisobutyronitrile (AIBN)/benzoyl peroxide (BPO) (initiator), with these components reoccurring continuously in literature (Figure 7) [78-81]. 
<smiles>C=C(C)C(=O)O</smiles><smiles>C=C(C)C(=O)OCCOC(=O)C(=C)CCCCC</smiles><smiles>C=CC(=O)O</smiles>

Acrylic acid<smiles>C=Cc1ccccc1C=C</smiles>

DVB<smiles>C=C(C)C(=O)OCC(CC)(CC)COC(=O)C(=C)C</smiles>

TMPTM<smiles>C=C(C)C(=O)OC</smiles><smiles>CC(C)(C#N)N=NC(C)(C)C#N</smiles>

AIBN<smiles>O=C(OOC(=O)c1ccccc1)c1ccccc1</smiles>

Figure 7. Chemical structures of the most common functional monomers, crosslinkers, and initiator molecules.

One differing consideration in the generation of MIPs is the polymerization approach selected to produce these synthetic receptors. The driving force behind this consideration is the particles size and homogeneity of the MIPs made [82, 83]. The most common approach is bulk polymerization, generating a monolithic polymer that can be mechanically ground and the template extracted by means of Soxhlet extraction with strong polar solvents [84]. Though this is the easiest method to synthesize MIPs it is also the most heterogeneous, yielding massively varying particle size, shapes and affinities 
to the template molecule $[85,86]$. Methodologies that facilitate the generation of smaller particles are therefore becoming more appealing, with the use of micro-emulsions and precipitation polymerization on the rise (figure 8) [87, 88]. These approaches can achieve particles size sub-micron, and yield more homogenous structure [89]. An increased mass transfer between MIP and target analyte makes these nano-sized MIPs worthwhile, even if their synthesis is more complex. This said, all these methods are currently considered limited, and lack scalability in an industrial setting.

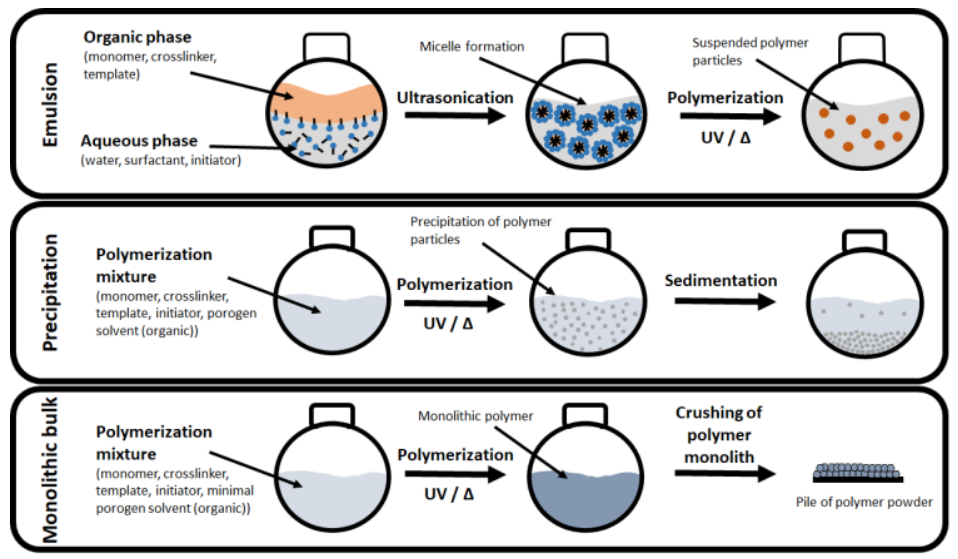

Figure 8. An artistic interpretation of the differing (emulsion, precipitation, and monolithic bulk) polymerization approaches.

One approach that has shown potential is the solid-phase synthesis of MIPs, producing water dispersible nanoMIPs that are highly homogenous and demonstrate great sensitivity and selectivity towards the template molecule [90]. The method used to synthesize these nanostructures is also potentially industrially scalable, fixing a template molecule to a functionalized support and introducing a polymerization mixture before activation with a UV source. Low affinity MIP and unreacted material is then washed off the solid support with cold water, whereas the high affinity MIPs are collected by subjecting the glass beads to hot water [91, 92]. Thus, highly reproducible water dispersed nanoMIPs are yielded. The scope of MIPs is vast, with any molecule of a molecular weight $<1000 \mathrm{D}$ being a potential target for traditionally synthesized MIPs and large peptides with the advancements of solid phase synthesis [93]. 


\section{Readout platforms}

Development of MIP based chemical sensors does not fall solely on the advancements of the polymeric receptors, but the adaptation and expansion of readout platforms that are capable of utilizing these receptors is another key factor [94]. MIPs have been integrated into many readout platforms but the most notable combinations are those with a QCM, electrochemical impedance, thermal techniques, and optical platforms [95-97]. Each sensory platform has its own benefits and drawbacks, with each performing optimal in a defined setting [98-102].

\section{Quartz crystal microbalance (QCM)}

As previously stated with the DNA based biosensors, a QCM consists of a thin piezoelectric crysal placed between electrodes. In brief, when a mass is applied across the piezoelectric crystal the resonance of the crystal is dampened (figure 9), and this effect can be mathematically equated using the Sauerbrey equation [103]. This platform facilitates the highly sensitive monitoring of mass across the crystal surface, opening the possibility of functionalising the crystal with MIPs and monitoring binding interactions as a change in mass. This approach has been successful for the detection of small molecules, nanoparticles, bacteria and proteins [104-107]. Issues arise from the deposition of the MIP layer, with methods currently lacking in reproducibility.

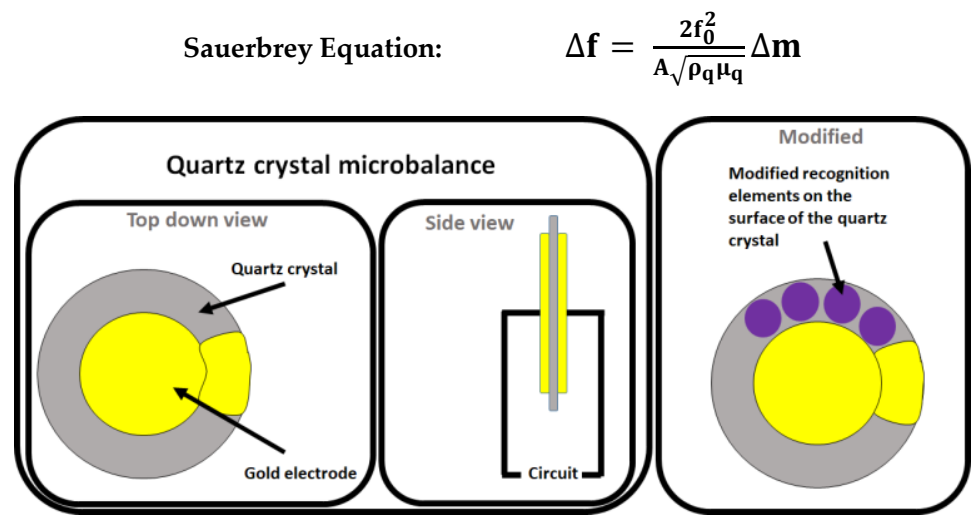

Figure 9. Quartz crystal microbalance (QCM) concept demonstrating an unmodified version and a modified version with a receptor layer on the quartz crystal. 


\section{Electrochemical impedance}

Electrochemical impedance is derived from the classical notion of ohm's law. Ohm's law states that the resitance (R) in an electrical system is a product of the voltage (v) devided by that of the current (I ) [108]. This relationship only proves to be true in ideal scenarios, requiring a more broad term known as impedance that factors in the more complex components that are present in the real world. It is common place to measure electrochemical impedance by applying an alternating current (AC) potential across an electrochemical cell and measuring the change in current (I) as a function of time. This concept has been extended towards the use in biosensors as receptor layers can be placed within these electrochemical cells, measuring the electrical impedance across the receptors [109]. Binding events at the receptor layers cause a shift in the electrochemical impedance verifying the presence of a target analyte (Figure 10) [110].

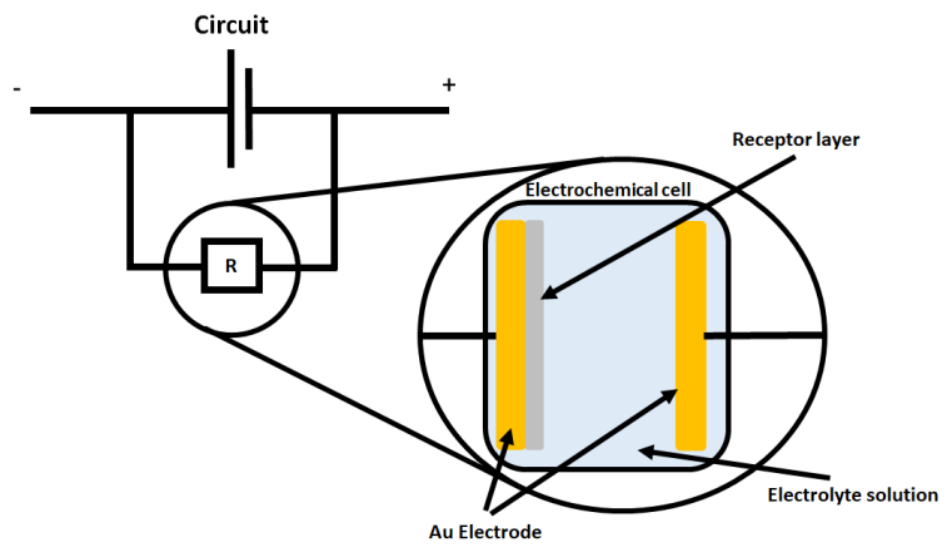

Figure 10. Principle behind an electrochemical impedance cell, where binding events at the receptor layer changes the electrical resistance of the cell and therefore the current that can pass through the circuit.

\section{Thermal readout techniques}

Unlike electrochemical or QCM readout techniques, thermal readout platforms quantify a binding event at the surface of a MIP as a change in temperature or thermal resistance across a phase interface [111]. The most promising example of this methodology is the platform reffered to as the "Heat Transfer Method" (HTM), where the MIP is deposited on a substrate between a heat source below 
and a liquid phase above. The heat source is set to a controlled temperature, where the flow of heat travels through the MIP coated substrate and into the liquid phase. A thermal couple is placed in both the heat source and the liquid phase enabling the temperatures to be monitored. As an analyte binds to the surface of the MIP, the thermal pathway between the heat source and the liquid phase is impeded. This increase in thermal resistance across the phase boundary can be seen as a change in observed temperature in the liquid phase $[112,113]$. This technique was originally developed for the detection of DNA hybridization as DNA strands have a larger molecular weight and therefore provide ample thermal resistance upon binding (Figure 11) [114]. The association of MIPs with this platform came later, with many examples of analytical targets being monitored with the readout platform. Most notably the platform has excelled at the detection of bacteria, small molecules (neurotransmitters), proteins, and other disease markers [115-120]. As with the other readout platforms discussed prior, the main drawback of this technique currently lies in the deposition of the MIP receptor layer and therefore lacks sensitivity and reproducibility in most cases.

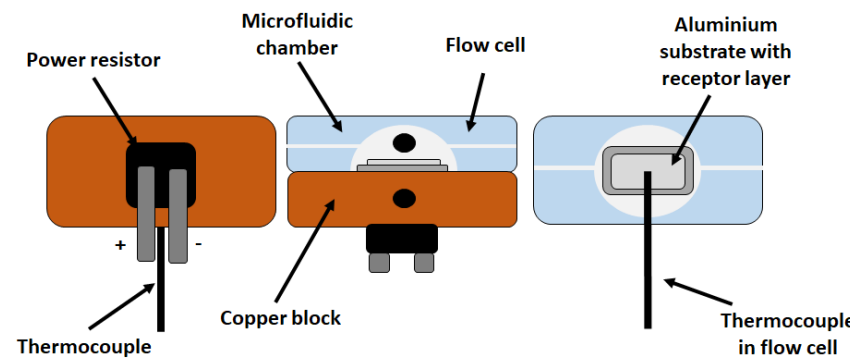

in copper

block

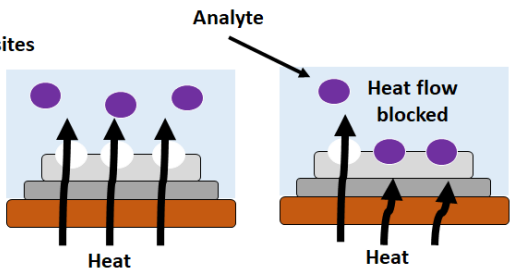

Figure 11. An illustration of the "Heat Transfer Method" analytical setup, demonstrating the flow cell used during the measurement process, and the thermal principle behind the technique. 


\section{Optical platforms}

Different optical properties can be intergreated into MIPs opening the possibility of using optical readout platforms to monitor the binding of a target analyte. The optical readout platform selected for analysis depends upon the modification that has been made to the MIP, which could include (but not limited to) the introduction of fluorophores, chromophores, or nanoparticles [121]. These alterations promote an optical response upon the binding of a target analyte, be it in the form of a quenching or enahcement type mechanism (Figure 12) [122-126]. Spectrophotometers are common place in the analysis of said binding events, though some modifications may allows for naked-eye detection of analytes (such as the introduction of chromophores) [127, 128].

\section{Quenching mechanism}

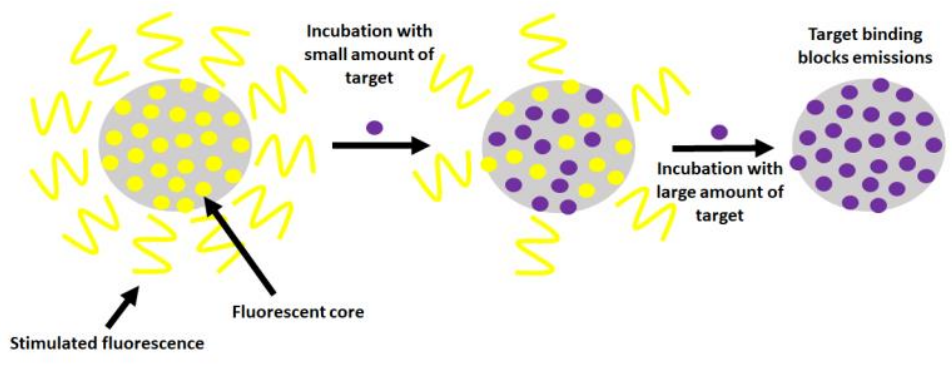

Figure 12. An example of a MIP backed fluorescence quenching mechanism, where the binding of a target analyte impedes the stimulated fluorescent emmisions.

Identifying areas of a methodology which can be improved upon is the key to merging MIPs into chemical sensors more harmoniously, expanding these platforms and making them more reliable [129]. Sensory and readout platforms utilizing MIPs therefore have room to develop further, integrating MIPs in new and unique ways as their application demands. Furthering this, there is the potential to use MIPs as both receptor and transducer, removing the need for electrical based chemical sensors all together. 


\section{Aims and outlines}

In this thesis, the synthesis and application of MIP-based sensory platforms will be investigated. Integrating MIPs as recognition elements in transducer dependent and independent strategies.

In Chapter 2 the current applications of MIPs in low-cost sensors and assay formats is reviewed. Analyzing the field for the latest innovations in terms of imprinting techniques and readout technologies that shows promise in a research setting and could be of potential value in a commercial setting. Aiming to identify areas of the current landscape that can be built upon, and has potential to areas that are not yet investigated. This chapter extended on the methodologies mentioned in the opening chapter, taking a deep dive into how these readout technologies play a part in the field of MIP-based sensing platforms.

Of the methods outlined in the chapter 2 the "heat transfer method" (HTM) was identified as a platform of interest, offering a simplistic method (thermal) of transducing a binding event at the MIPs surface into a tangible signal. The technology has shown promise in the sensing of bacterial species, alongside the detection of neurotransmitters in the past. Therefore, in Chapter 3 the use of the HTM in the sensing of New Psychoactive Substances (NPS) is proposed, offering a cheap and rapid way of detecting novel illicit substances. The work presented builds develops the implementation of MIPs as the polymeric receptor layer, taking a direct surface grafting approach opposed to the implementation of the MIPs via a polymeric adhesive. Thus, the sensitivity of the platform is assessed in comparison with the standard deposition protocol, optimizing layer thickness, and polymerization time for the best possible LoD.

The development of rapid sensing with MIPs does not have to require the coupling of an bulky readout methodology. Considering the use of traditional sensing platforms that utilize lateral flow assays, ELISA, testing strips and more for colorimetric verification, they do not rely on bulky readout technologies for in-the-field-testing. The approach was therefore investigated in chapter 4, designing a MIP-based dye displacement assay that would allow visual verification of a target's presence. The boon of this setup being the lack of electrical components, reducing the size and therefore weight of the MIPbased sensor. By producing a powder that can selectively bind a target and provide an instant colorful visual confirmation is a technology that can be understood and interpreted by anyone. This concept therefore has high value 
for in-the-field-testing as equipment is limited, making it ideal for the rapid screening of compounds in numerous situations.

Previously, the MIP-based dye displacement assay was designed for the rapid detection of NPS, but such an assay would be of benefit for the detection of traditional drug compounds. Chapter 5 extends the concept towards the detection of amphetamine, widening the proof-of-concept from a model NPS to a compound that is widely known and abused. The context in which the assay is used was further extended by using the platform for the rapid analysis of amphetamine spiked urine samples. Highlighting the colorimetric nature of the assay is persistent even in a "complex" liquid matrix.

Substances of abuse are not the only compounds that have social impact, environmental contaminents are a key area of research that affect us all. In particular, the presence of antibiotics in the environment is an increasing issue, with the increased concentrations of these compounds leading to resistant bacterial strains that threaten the very basis of health care. Developing lowcost rapid sensors that are capable of detecting antibiotics in waste, food products and environmental samples is therefore essential in regaining some control of the amount fo these substances unknowingly released into the wild. Chapter 6 attempts to provide a MIP based olution for this crisis, tailoring the aforementioned dye displacement assay towards the antibiotic amoxicillin. The research presented in this chapter offers a more elegant homogeneous method of MIP synthesis, adopting emulsion polymerization for the generating of microspherical MIP particles. The complex structural nature of amoxicillin in comparison to prior targets enables a comparison of potential complimentary functional monomers, incorporating intelligent compositional design to compliment the more sophisticated polymerization technique. Expansion of potential dye molecules are also discussed in this paper, highlighting the use of mordant orange as a reporting molecule.

Finally, chapter 7 summarizes the research outlined throughout the thesis, discussing and scrutinizing the results obtained. 


\section{Chapter 1 References}

[1] Stetter, J. R.; Penrose, W. R. Understanding chemical sensors and chemical sensor arrays (electronic noses): Past, present, and future. Sesnors Update. 2002, 10, 189-229.

[2] Xu, H.; Akbari, M. K.; Kumar, S.; Verpoort, F.; Zhuiykov, S. Atomic layer deposition state-of-the-art approach to nanoscale hetero-interfacial engineering of chemical sensors electrodes: A review. Sensors \& Actuators B: Chem. 2021, 331, 129403.

[3] Norizan, M, N.; Moklis, M. H.; Demon, S. Z. N.; Halim, N. A.; Samsuri, A.; Mohamad, I. S.; Knight, V. F.; Abdullah, N. Carbon nanotubes: functionalization and their application in chemical sensors. RSC Adv. 2020, 71, 43704-43732.

[4] Wu, L.; Wang, Y.; Xu, X.; Liu, Y.; Lin, B.; Zhang, M.; Zhang, J.; Wan, S.; Yang, C.; Tan, W. Ampamer-based detection of circulating targets for precision medicine. Chem Rev. 2021.

[5] Berneira, L. M.; Ritter, M.; da Silva, C. C.; Poletti, T.; Passos, L. F.; da Rosa, B. N. dos Santos, M. A. Z.; de Pereira, C. M. P. Extraction and identification of formulations of anabolic androgenic steroids: A forensic educational approach. Quim, Nova. 2020, 43(10), 1505-1509.

[6] Schram, J.; Parrilla, M.; Sleegers, N.; van Durme, F.; van den Berg, J.; van Nuijs, A. L. N.; de Wael, K. Electrochemical profiling and liquid chromatography-mass spectrometry characterization of synthetic cathinones: From methodology to detection in forensic samples. Drug Testing and Anal. 2021, 13(7), 1282-1294.

[7] Arnaud, C. H. Glucose meter-based device detects pathogens. Chem \& Eng News. 2021, 99(6), 5.

[8] Morf, W. E. Glass electrodes, Studi Anal Chem. 1981, 2, 337-374.

[9] Bakker, E.; Pretsch, E.; Buhlmann, P. Selectivity of potentiometric ion sensors. Anal. Chem. 2000, 72(6), 1127-1133.

[10] Hideyasu, N.; Satoko, T.; Youichi, S. A cobalt-nickel metal-alloy thin-film sensor for hydrogen-phosphate ion. Anal. Sci. 2021, 37(2), 337-340.

[11] Cheong, Y. H.; Ge, L.; Lisak, G. Highly reproducible solid contact ion selective electrodies: Emerging opportunities for potentiometry - A review. Anal. Chim. Acta. 2021, 1162, 338304

[12] Yag, L. S.; Bao, Y. J.; Hai, X. Z.; Kun, W.; Jing, L.; Jie, H.; Zhen-yue, Z.; Biao, J. Preparation and properties of a novel all-solid-state ammonium ion selective electrode. Xiandai Huagong. 2020, 40(12), 237-240.

[13] Yuan, X.; Zhang, X.; Huang, Y.; Jie, J.; Wei, Q.; Tan, M.; Yu, Y. Development of an electrochemical sensor for chloride ion detection using ion-sensitive field-effect transistor array. Int. J. Electrochem. Sci. 2021, 16, 150911.

[14] Myers, R. J. One-hundred years of pH. J. Chem. Educ. 2010, 87(1), 30-32.

[15] Shushan, A.; Kosloff, M. Structural design principles for specific ultra-high affinity interactions between collicins/pyocins and immunity proteins. Sci. Reports. 2021, 11(1), 3789.

[16] Lan, Y.; Hiebner, D. W.; Casey, E. Self-assembley and regeneration stratergy for mitigation of membrane biofouling by the exploitation of enzymatic nanoparticles. Chem. Eng. J. 2021, 412, 128666. 
[17] Dahlman, C. J.; Kennard, R. M.; Paluch, P.; Venkatesan, N. R.; Chabinyc, M. L.; Manjunatha Reddy, G. N. Dynamic motion of organic spacer cations in RuddlesdenPopper lead iodide perovskites probed by solid-state NMR spectroscopy. Chem Mater. 2021, 33(2), 642-656.

[18] Schou, C.; Heegaard, N. H. H. Recent applications of affinity interactions in capillary electrophoresis. Electrophoresis. 2006, 27(1), 44-59.

[19] Wang, Y.; Maspoch, D.; Zou, S.; Schatz, G. C.; Smalley, R. E.; Mirkin, C. A. Controlling the shape, orientation, and linkage of carbon nanotube features with nano affinity templates. PNAS. 2006, 103(7), 2026-2031.

[20] Rudkevich, D. M.; Zyryanov, G. V. Solid-state materials and molecular cavities and containers for the supramolecular recognition and storage of NOX-species: A review. Inorg Chem. 2015, 35(3), 128-178.

[21] Ahmad, H. M. N.; Dutta, G.; Csoros, J.; Si, B.; Yang, S.; Halpern, J. M.; Seitz, W. R.; Song, E. Stimuli-responsive template polymer as a target receptor for a conformationbased electrochemical sensing platform. ACS Appl. Polym. Mater. 2021, 3(1), 329-341.

[22] Weiki, T. R.; Paul, F. Conformational selection in protein binding and function. Protein Sci. 2014, 23(11), 1508-1518.

[23] Chen, X.; Li, J. Bioinspired by cell membranes: Functional polymeric materials for biomedical applications. Mater. Chem. Front. 2020, 4, 750-774.

[24] Messina, G. M. L.; De Zotti, M.; Lettieri, R.; Gatto, E.; Venanzi, M.; Formaggio, F.; Toniolo, C.; Marletta, G. Design of lipidic platforms anchored within nanometric cavities by peptide hooks. RSC Adv. 2016, 6, 46984-46993.

[25] Vegas, V. G.; Beobide, G.; Castillo, O.; Reyes, E.; Gomez-arcia, C. J.; Zamora, F.; AmoOchoa, P. A bioinspired metal-organic approach to corss-linked functional 3D nanofibers hydro- and aero-gels with effective mixture separation of nucleobases by molecular recognition. Nanoscale. 2020, 12, 14699-14707.

[26] Steiner, S.; Sotzny, F.; Bauer, S.; Na, I.; Schueck-Henneresse, M.; Corman, V. M.; Schwarz, T.; Drosten, C.; Wendering, D. J.; Behrends, U.; Volk, H.; Scheibenbogen, C.; Hanitsch, L. HCoV- and SARS-CoV-2 cross-reactive T cells in CVID patients. Front. Immun. 2020, 11, 607918.

[27] Ying, Q.; Yongpeng, X.; Yuanfng, S.; Na, Y.; Kexi, L. Recent progress in immune response and immunotherapy of acinetobacter beumannii infection. Yixue Zongshu. 2020, 26(20), 59-64.

[28] Lindashl, G. Subdominance in antibody responses: implications for vaccine development. Micro. Mol. Bio. Rev. 2021, 85(1), e00078.

[29] Tamaki, F. K. Directed evolution of enzymes. Emerg Top Life Sci. 2020, 4(2), 119-127.

[30] Newman, J. D.; Turner, A. P. F. Home blood glucose biosensors: A commercial perspective. Biosensors and Bioelectronics. 2005, 20(12), 2435-2453.

[31] Tonyushkina, K.; Nichols, J. H. Glucose meters: A review of technical challenges to obtaining accurate results. J Diabetes Sci Tech. 2009, 3(4), 971-980.

[32] Wilson, K. G.; Ovington, P.; Dean, D. A low-cost inkjet-printed glucose test strip system for resource-poor settings. J diabetes Sci Technol. 2015, 9(6), 1275-1281.

[33] Koczula, K. M.; Gallotta, A. Lateral flow assays. Essays Biochem. 2016, 60(1), 111-120. 
[34]Latman, N. S.; Bruot, B. C. Evaluation of home pregnancy test kits. Biomed Instr Tech. 1989, 23(2), 144-149.

[35] Parker, D. Tumour targeting with radiolabeled macrocycle-antibody conjugates. Chem. Soc. Rev. 1990, 19, 271-291.

[36] Aydin, S. A short history, principles, and types of ELISA, and our laboratory experience with peptide/protein analyses using ELISA. Peptides. 2016, 72, 4-15.

[37] Murdock, R. C.; Shen, L.; Griffin, D. K.; Kelley-Loughnane, N.; Papautfky, I.; Hagen, J. A. Optimization of a paper-based ELISA for a human performance biomarker. Anal. Chem. 2013, 85(23), 11634-11642.

[38] Xiong, Y.; Leng, Y.; Li, X.; Huang, X.; Xiong, Y. Emerging strategies to enhance the sensitivity of competitive ELISA for detection of chemical contaminants in food samples. TrAC trends in Anal Chem. 2020, 126, 115861.

[39] Zhou, F.; Wang, M.; Yuan, L.; Cheng, Z.; Wu, Z.; Chen, H. Sensitive sandiwch ELISA baed on a gold nanoparticle layer for cancer detection. Analyst. 2012, 137, 1779-1784.

[40] Folloni, S.; Bellocchi, G.; Kagkli, D.; Ptor-Benito, S.; Aguilera, M.; Mazzeo, A.; Querci, M.; Eede, G.; Ermolli, M. Development of an ELISA reverse-based assay to assess the presence of mycotoxins in cereal flour. Food Anal Methods. 2011, 4, 221-227.

[41] Mehrotra, P. Biosensors and their applications - A review. J oral Bio \& craniofacial res. 2016, 6, 153-159.

[42] Wang, J. DNA biosensors based on peptide nucleic acid (PNA) recognition layers. A review. Biosensors \& Bioelectronics. 1998, 13, 757-762.

[43] Kaewphinit, T.; Santiwatanakul, S.; Promptmas, C.; Chansiri, K. Detection of nonamplified mycobacterium tuberculosis genomic DNA using piezoelectric DNA-based biosensors. Sensors. 2010, 10(3), 1846-1856.

[44] Campbell, N. F.; Evans, J. A.; Fawcett, N. C. Detection of poly (U) hybridization using azido modified poly (A) coated piezoelectric crystals. BioChem Biophys Res Commun. 1993, 196(2), 858-863.

[45] Saidur, M. R.; Abdul Aziz, A. R.; Basirun, W. J. Recent advances in DNA-based electrochemical biosensors for heavy metal ion detection: A review. Biosensors $\mathcal{E}$ Bioelectronics. 2017, 90, 125-139.

[46] Xie, B.; Ramanathan, K.; Danielsson, B. Mini/micro thermal biosensors and other related devices for biochemical/clinical analysis and monitoring. TrAC trends in anal chem. 2000, 19(5), 340-349.

[47] Huang, Y.; Yang, H. Y.; Ai, Y. DNA single-base mismatch study using graphene oxide nanosheets-based fluorometric biosensors. Anal. Chem. 2015, 87(18), 9132-9136.

[48] Mozaev, V. V.; Khmelnitsky, Y. L.; Sergeeva, M. V.; Belova, A. B.; Klyachko, N. L.; Levashov, A. V.; Martinek, K. Catalytic activity and denaturation of enzymes in water/organic cosolvent mixtures. Euro J. BioChem. 1989, 184(3), 597-602.

[49] Bermudez, O.; Forciniti, D. Aggregation and denaturation of antibodies: A capillary electrophoresis, dynamic light scattering, and aqueous two-phase partitioning study. J. Chroma B. 2004, 807, 17-24.

[50] Fan, Y.; Zeng, G.; Ma, X. Effects of prepolymerization on surface molecularly imprinted polymer for rapid separation and analysis of sulfonamides in water. J. Colloid E Interface Sci. 2020, 571, 21-29. 
[51] Peeters, M.; Troost, F. J.; Mingels, R. H. G.; Grinsven, B.; Vranken, T.; Ingebrandt, S.; Thoelen, R.; Cleij, T. J.; Wagner, P. Impedimetric Detection of Histamine in Bowel Fluids Using Synthetic Receptors with pH-Optimized Binding Characteristics. Anal Chem. 2013, 85, 1475-83.

[52] Jupai, J.; Razali, M.; Buyuktiryaki, S.; Kecili, R.; Szekely, G. Long-term stability and reusability of molecularly imprinted polymers. Polym Chem. 2017, 8(4), 666-673.

[53] Wackers, G.; Putzeys, T.; Peeters, M.; Cauter, L.; Cornelis, P.; Wubbenhorst, M.; Tack, J.; Troost, F.; Verhaert, N.; Doll, T.; Wagner, P. Towards a catheter-based impredimetric sensor for the assessment of instestinal histamine levels in IBS patients. Biosensors $\mathcal{E}$ Bioelectronics. 2020, 158, 112152.

[54] Cheng, J.; Li, Y.; Zhong, J.; Lu, Z.; Wang, G.; Sun, M.; Jiang, Y.; Zou, P.; Wang, X.; Zhoa, Q.; Wang, Y.; Rao, H. Molecularly imprinted electrochemical sensor based on biomass carbon decorated with $\mathrm{MOF}$-derived $\mathrm{Cr}_{2} \mathrm{O}_{3}$ and silver nanoparticles for selective and sensitive detection of nitrofurazone. Chem. Eng. J. 2020, 398, 125664.

[55] Kajisa, T.; Sakata, T. Molecularly imprinted artificial biointerface for an enzyme-free glucose transistor. ACS Appl. Mater. Interfaces. 2018, 41, 34983-34990.

[56] Qian, K.; Deng, Q.; Fang, G.; Wang, J.; Pan, M.; Wang, S.; Pu, Y. Metal-organic frameworks supported surface-imprinted nanoparticles for the sensitive detection of metolcarb. Biosensors \& Bioelectronics. 2016, 79, 359-363.

[57] Jenik, M.; Seifner, A.; Krassnig, S.; Seidler, K.; Lieberzeit, P. A.; Dickert, F. L.; Jungbauer, C. Sensors for bioanalytes by imprinting-polymers mimicking both biological receptors and the corresponding bioparticles. Biosensors \& Bioelectronics. 2019, 25, 9-14.

[58] Battal, D.; Akgonullu, S.; Yalcin, M. S.; Yavuz, H.; Denizli, A. Molecularly imprinted polymer based quartz crystal microbalance sensor system for sensitive and label-free detection of synthetic cannabinoids in urine. Biosensors \& Bioelectronics. 2018, 111, 10-17.

[59] Mudd, S. A hypothetical mechanism of antibody formation. J of Immunology. 1932, 23, 423-427.

[60] Polyakov, M. V.; Stadnik, P.; Paryckij, M.; Malkin, I.; Duchina, F. On the structure of silica. Zhurnal Fizieskoj Kihimii. 1993, 4, 454-456.

[61] Tang, Y.; Lan, J.; Gao, X.; Liu, X.; Xhang, D.; Wei, L.; Gao, Z.; Li, J. Determination of clenbuterol in pork and potable water samples by molecularly imprinted polymer through the use of covalent imprinting method. Food Chem. 2016, 190, 952-959.

[62] Qi, P.; Wang, J.; Wang, L.; Li, y.; Jin, J.; Su, F.; Tian, Y.; Chen, J. Molecularly imprinted polymers synthesized via semi-covalent imprinting with sacrificial spacer for imprinting phenols. Polymer. 2010, 51, 5417-5423.

[63] Yusof, N. N. M.; Tanioka, E.; Kobayashi, T. Molecularly imprinted polymer particles having coordination hydrogen bonding in covalent-imprinting for efficient recognition towards vanillin. Separation \& Purification Tech. 2014, 122, 341-349.

[64] Kempre, M.; Mosbach, K. Binding studies on substrate- and enantio-selective molecularly imprinted polymers. Anal Biochem Clinical Anal. 1991, 24, 1137-1145.

[65] Karim, K.; Breton, F.; Rouillon, R.; Piletska, E. V.; Guerreiro, A.; Chianella, I.; Piletsky, S. S. How to find effective functional monomers for effective molecularly imprinted polymers? Adv Drug Delivery Rev. 2005, 57, 1795-1808. 
[66] Davies, M. P.; Biasi, V.; Perrett, D. Approaches to the rational design of molecularly imprinted polymers. Anal Chimi Acta. 2004, 504, 7-14.

[67] Cornel, E. J.; Jiang, J.; Chen, S.; Du, J. Principles and characteristics of polymerizationinduced self-assembly with various polymerization techniques. CCS Chem. 2021, 3(4), 2104-2125.

[68] Nezhad-Mokhtari, P.; Ghorbani, M.; Roshangar, L.; Rad, J. S. A review on the construction of hydrogel scaffolds by various chemically techniques for tissue engineering. European Poly J. 2019, 117, 64-76.

[69] Ansari, S.; Masoum, S. Molecularly imprinted polymers for capturing and sensing proteins: Current progress and future implications. TrAC Trends Anal Chem. 2019, 114, 2947.

[70] Ndunda, E. N. Molecularly imprinted polymers - A closer look at the control polymer used in determining the imprinting effect: A mini review. J Mol Recognit. 2020, 33(11), e2855.

[71] Hermawan, B. A.; Mutakin.; Hasanah, A. N. Role of porogenic solvent type on the performance of a monolithic imprinted column. Chemical Papers. 2021, 75, 1301-1311.

[72] Foroughirad, S.; Haddadi-Asl, V.; Khosravi, A.; Salami-Kalajahi, M. Effect of porogenic solvent in synthesis of mesoporous and microporous molecularly imprinted polymer based on magnetic halloysite nanotubes. Mater Сотти. 2021, 26, 101780.

[73] Fuchs, Y.; Soppera, O.; Haupt, K. Photopolymerization and photostructuring of molecularly imprinted polymers for sensor applications - a Review. Anal Chimi Acta. 2012, 717, 7-20.

[74] Yagci, Y.; Jockusch, S.; Turro, N. J. Photoinitiated polymerization: Advances, challenges, and opportunities. Macromolecules. 2010, 43(15), 6245-6260.

[75] Acquah, C.; Danquah, M. K.; Moy, C. K. S. ; Ongkudon, C. M. In-progress thermochemical analysis of in situ poly(ethylene glycol methacrylate-co-glycidyl methacrylate) monolithic absorbent synthesis. J Appl Polym Sci. 2016, 133(22), 43507.

[76] Panagiotopoulou. M.; Beyazit, S.; Nestora, S.; Haupt, K.; Bui, B. T. S. Initiator-free synthesis of molecularly imprinted polymers by polymerization of self-initiated monomers. Polymers. 2015, 66, 43-51.

[77] Li, Y.; he, W.; Peng, Q.; Hou, L.; He, J.; Li, K. Aggregation-induced emission luminogen based molecularly imprinted ratiometric fluorescence sensor for the detection of rhodamine 6G in food samples. Food Chem. 2019, 287, 55-60.

[78] Golker, K.; Olsson G. D.; Nicholls, I. A. The influence of a methyl substituent on molecularly imprinted polymer morphology and recognition - Acrylic acid versus methacrylic acid. European Polymer J. 2017, 92, 137-149.

[79] Prabakaren, K.; Jandad, P. J.; Luo, J.; Fu, C.; Wei, Q. Molecularly imprinted poly(methacrylic acid) based QCM biosensor for selective determination of L-tryptophan. Coll Surf A. 2021, 611, 125859.

[80] Wang, Z.; Yuan, D.; Fu, D. Preparation and property study of molecularly imprinted polymers for methyl orange. Fenxi Shiyanshi. 2017, 36(3), 306-311.

[81] Riskin, M.; Ben-Amram, Y.; Tel-Vered, R.; Chegel, V.; Almog, J; Willner, I. Molecularly imprinted $\mathrm{Au}$ nanoparticles composites on $\mathrm{Au}$ surfaces for the surface 
plasmon resonance detection of pentaerythritol tetranitrate, nitroglycerin, and ethylene glycol dinitrate. Anal. Chem. 2011, 83, 3082-3088.

[82] El-Schich, Z.; Zhang, Y.; Feith, M.; Beyer, S.; Sternbrek, L.; Ohisson, L.; Stollenwerk, M.; Wingren, A. G. Molecularly imprinted polymers in biological applications. BioTech. 2020, 69(6).

[83] Fresco-Cala, B.; Batista, A. D.; Cardenas, S. Molecularly imprinted polymer Microand Nano-particles: A review. Molecules. 2020, 25, 4740.

[84] Liang, J.; Xiang, B.; He, Q.; Chen, L.; Zhang, H. Preparation and properties of double template molecularly imprinted polymers with phthalate isomers. Harbin Gongye Daxue Хиевао. 2020, 52(11), 52-58.

[85] Wloch, M.; Datta, J. Synthesis and polymerisation techniques of molecularly imprinted polymers. Compr Anal Chem. 2019, 86, 17-40.

[86] Retnaningtyas, Y.; Supriyanto, G.; Nyoman Tri Puspaningsh, N.; Irawan, R.; Siswodihardjo, S. A novel molecular imprinted polymer for the selective adsorption of Darabinitol from spiked urine. Turk J. Chem. 2020, 44(5).

[87] Pardeshi, S.; Singh, S. K. Precipitation polymerization: A versatile tool for preparing molecularly imprinted polymer beads for chromatography applications. RSC Adv. 2016, 6, 23525-23536.

[88] Wei, S.; Molinelli, A.; Mizaikoff, B. Molecularly imrpinted micro and nanospheres for the selective recognition of 17ß-estradiol. Bioesnsors and Bioelectronics. 2006, 21, 1943-1951.

[89] Dai, C. M.; Zhou, X. F.; Zhang, Y. L.; Liu, S. G.; Zhang, J. Synthesis by precipitation polymerization of molecularly imprinted polymer for the selective extraction of diclofenac from water samples. J of Hazardous Materials. 2011, 198, 175-181.

[90] Poma, A.; Guerreiro, A.; Whitcombe, M. J.; Piletska, E. V.; Turner, A. P. F.; Piletsky, S. A. Solid-phase synthesis of molecularly imprinted polymer nanoparticles with a reusable template-“Plastic Antibodies". Adv. Funct. Mater. 2013, 23, 2821-2827.

[91] Canfarotta, F.; Poma, A.; Guerreiro, A.; Piletsky, S. Solid-phase synthesis of molecularly imprinted nanoparticles. Nat. Protoc. 2016, 11, 443-445.

[92] Ambrosini, S.; Beyazit, S.; Haupt, K.; Bui, B. T. S. Solid-phase synthesis of molecularly imprinted nanoparticles for protein recognition. Chem. Commun. 2013, 49, 6746-6748.

[93] Xu, J.; Medina-Rangel, P. X.; Haupt, K.; Bui, B. T. S. Chapter six - guide to the preparation of molecularly imprinted polymer nanoparticles for protein recognition by solid phase synthesis. Methods in Enzymology. 2017, 590, 115-141.

[94] Feng, F.; Zheng, J.; Qin, P.; Han, T.; Zhao, D. A novel quartz crystal microbalance sensor array based on molecularly imprinted polymers for simultaneous detection of clenbuterol and its metabolites. Talanta. 2017, 167, 94-102.

[95] Motla, S.; Bouchikhi, B.; Llobet, E.; Bari, E. Synthesis and characterization of a highly sensitive and selective electrochemical sensor based on molecularly imprinted polymer with gold nanoparticles modified screen-printed electrode for glycerol determination in wastewater. Talanta. 2020, 216, 120953.

[96] Eersels, K.; Grinsven, B.; Ethirajan, A.; Timmermans, S.; Jimenez-Monroy, K. L.; Bogie, J. F. J.; Punniyakoti, S.; Vandenryt, T.; Hendriks, J. J. A.; Cleij, T. J.; Daemen, M. J. A. P.; Somers, V.; Ceuninck, W.; Wagner, P. Selective identification of macrophages and cancer cells based on thermal transport through surface-imprinted polymer layers. ACS App Materials \& Interfaces. 2013, 5, 7258-7267. 
[97] Naklua, W.; Suedee, R.; Lieberzeit, P. A. Dopaminergic receptor-ligand binding assay based on molecularly imprinted polymers on quartz crystal microbalance sensors. Biosensors \& Bioelectronics. 2016, 81, 117-124.

[98] Ma, X.; He, X.; Li, W.; Zhang, Y. Epitope molecularly imprinted polymer coated quartz crystal microbalance sensor for the determination of human serum albumin. Sensors $\mathcal{E}$ Actuators B: Chem. 2017, 248, 879-886.

[99] Eersels, K.; Grinsven, B.; Peeters, M.; Cleij, T. J.; Wagner, P. Heat transfer as a new sensing technique for the label-free detection of biomolecules. Label-Free Biosensing. 2017, 383-407.

[100] Betlem, K.; Down, M. P.; Foster, C. W.; Akthar, S.; Eersels, K.; Grinsven, B.; Cleij, T. J.; Banks, C. E.; Peeters, M. Development of a flexible MIP-based biosensor application for the thermal detection of neurotransmitters. MRS Adv. 2018, 3, 1569-1574.

[101] Scheller, F. W.; Zhang, X.; Yarman, A.; Wollenberger, U.; Gyuresanyl, R. E. Molecularly imprinted polymer-based electrochemical sensors for biopolymers. Current Opinions in Electrochem. 2019, 14, 53-59.

[102] Wang, M.; Fu, Q.; Zhang, K.; Wan, Y.; Wang, L.; Gao, M.; Xia, Z.; Gao, D. A magnetic and carbon dot based molecularly imprinted composite for fluorometric detection of 2,4,6trinitrophenol. Microchim Acta. 2019, 186, 86.

[103] Diltemiz, S. E.; Kecili, R.; Ersoz, A.; Say, R. Molecular imprinting technology in quartz crystal microbalance sensors. Sensors. 2017, 17(3), 454.

[104] Yilmaz, E.; Majidi, D.; Ozgur, E.; Denizli, A. Whole cell imprinting based Escherichia coli sensors: A study for SPR and QCM. Sensors \& Actuators B: Chem. 2015, 209, 714-721.

[105] Gultekin, A.; Karanfil, G.; Kus, M.; Sonmezoglu, S.; Say, R. Preperation of MIP-based QCM nanosensor for detection of caffeic acid. Talanta. 2014, 119, 533-537.

[106] Cicek, C.; Yilmaz, F.; Ozgur, E.; Yavuz, H.; Denizli. Molecularly imprinted quartz crystal microbalance sensor (QCM) for Bilirubin detection. Chemosensors. 2016, 4(4), 21.

[107] Lieberzeit, P. A.; Jungmann, C.; Schranzhofer, L. Molecular imprinting on the nanoscale - rapid detection of Ag nanoparticles by QCM sensors. Procedia Eng. 2014, 87, 236-239.

[108] Chang, B.; Park, S. Electrochemical impedance spectroscopy. Annual Rev Anal Chem. 2010, 3, 207-229.

[109] Randviir, E.; Banks, C. E. Electrochemical impedance spectroscopy: an overview of bioanalytical applications. Anal methods. 2013, 5, 1098-1115.

[110] Hammond, J. L.; Formisano, N.; Estrela, P.; Carrara, S.; Tkac, J. Electrochemical biosensors and nanobiosensors. Essays Biochem. 2016, 60(1), 69-80.

[111] Grinsven, B.; Eersels, K.; Peeters, M.; Losada-perez, P.; Vandenryt, T.; Cleij, T. J.; Wagner, P. The heat-transfer method: A versatile low-cost, label-free, fast, and userfriendly readout platform for biosensor applications. ACS Appl. Mater. Interfaces. 2014, 6, 13309-13318.

[112] Eersels, K.; Grinsven, B.; Khorshid, M.; Somers, V.; Puttmann, C.; Stein, C.; Barth, S.; Dilien, H.; Bos, G. M. J.; Germeraad, W. T. V.; Cleij, T. J.; Thoelen, R.; Ceuninck, W.; Wagner, P. Heat-transfer-method-based cell culture quality assay through cell detection by surface imprinted polymers. Langmuir. 2015, 31, 2043-2050. 
[113] Eersels, K.; Grinsven, B.; Ethirajan, A.; Timmermans, S.; Jimenez-monroy, K. L.; Bogie, J. F. J.; Punniyakoti, S.; Vandenryt, T.; Hendriks, J. J. A.; Cleij, T. J.; Daemen, M. J. A. P.; Somers, V.; Ceuninck, W.; Wagner, P. Selective identification of macrophages and cancer cells based on thermal transport through surface-imprinted polymer layers. ACS Appl. Mater. Interfaces. 2013, 15, 7258-7267.

[114] Grinsven, B.; Bon, N. V.; Strauven, H.; Grieten, L.; Murib, M.; Jimenez Monroy, K. L.; Janssens, S. D.; Haenen, K.; Schoning, M. J.; Vermeeren, V.; Ameloot, M.; Michiels, L.; Theolen, R.; Ceuninck, W.; Wagner, P. Heat-transfer resistance at solid-liquid interfaces; A tool for the detection of single-nucleotide polymorphisms in DNA. ACS Nano. 2012, 6, 2712-2721.

[115] Redeker, E. S.; Eersels, K.; Akkermans, O.; Royakkers, J.; Dyson, S.; Nurekeyeva, K.; Ferrando, B.; Cornelis, P.; Peeters, M.; Wagner, P.; Dilien, H.; Grinsven, B.; Cleij, T. J. Biomimetic bacterial identification platform based on thermal wave transport analysis (TWTA) through surgace imprinted polymers. ACS infect Dis. 2017, 3(5), 388-397.

[116] Grinsven, B.; Eersels, K.; Akkermans, O.; Ellermann, S.; Kordek, A.; Peeters, M.; Deschaume, O.; Bartic, C.; Dilien, H.; Redeker, E. S.; Wagner, P.; Cleij, T. J. Label-free detection of Escherichia coli based on thermal transport through surface imprinted polymers. ACS Sens. 2016, 1(9), 1140-1147.

[117] Peeters, M.; Kobben, S.; Jimenez-monroy, K.; Modesto, L.; Kraus, M.; Vandenryt, T.; Gaulke, A.; Grinsven, B.; Ingebrandt, S.; Junkers, T.; Wagner, P. Thermal detection of histamine with a graphene oxide base molecularly imprinted polymer platform prepared by reversible addition-fragmentation chain transfer polymerization. Sensors \& Actuators $B$. 2014, 203, 527-535.

[118] Vandenryt, T.; Grinsven, B.; Eersels, K.; Cornelis, P.; Kholwadia, S.; Cleij, T. J.; Thoelen, R.; Ceuninck, W.; Peeters, M.; Wagner, P. Single-shot detection of neurotransmitters in whole-blood samples by means of the heat-transfer method in combination with synthetic receptors. Sensors. 2017, 17, 2701.

[119] Bers, K.; Eersels, K.; Grinsven, B.; Daemen, M.; Bogie, J. F. J.; Hendriks, J. J. A.; Bouwmans, E. E.; Puttmann, C.; Stein, C.; Barth, S.; Bos, G. M. J.; Germeraad, W. T. V.; Ceuninck, W.; Wagner, P. Heat-transfer resistance measurement method (HTM)-based cell detection at trace levels using a progressive enrichment approach with highly selective cell-binding surface imprints. Langmuir. 2014, 30, 3631-3639.

[120] Peeters, M.; Grinsven, B.; Cleij, T. J.; Jimenez-monroy, K. L.; Cornelis, P.; Perez-Ruiz, E.; Wackers, G.; Theolen, R.; Ceuninck, W.; Lammertyn, J.; Wagner, P. Label-free protein detection based on the heat-transfer method-A case study with the peanut allergen ara $\mathrm{h}$ 1 and amptamer-based synthetic receptors. ACS Appl. Mater. Interfaces. 2015, 19, 1031610323.

[121] Rico-Yuste, A.; Carrasco, S. Molecularly imprinted polymer-based hybrid materials for the development of optical sensors. Polymers. 2019, 11(7), 1173.

[123] Xiao, D.; Su, L.; Teng, Y.; Hao, J.; Bi, Y. Fluorescent nanomaterials combined iwth molecular imrpinting polymer: Synthesis, analytical applications, and challenges. Microchimica Acta. 2020, 187, 399. 
[124] Feng, S.; Hu, Y.; Ma, L.; Lu, X. Development of molecularly imprinted polymerssurface-enhanced raman spectroscopy/colorimetric dual sensor for determination of chlorpyrifos in apple juice. Sensors and Actuators B: Chem. 2017, 241, 750-757.

[125] Ye, T.; Yin, W.; Zhu, N.; Yuan, M.; Cao, H.; Yu, J.; Gou, Z.; Wang, X.; Zhu, H.; Reyihanguli, A.; $\mathrm{Xu}, \mathrm{F}$. Colorimetric detection of pyrethriod metabolite by using surface molecularly imprinted polymer. Sensors and actuators B: Chem. 2018, 254, 417-423.

[126] Zhao, B.; Feng, S.; Hu, Y.; Wang, S.; Lu, X. Rapid determination of atrazine in apple juice using molecularly imrpinted polymers coupled with gold nanoparticlescolorimetric/SERS dual chemosensor. Food Chem. 2019, 276, 366-375.

[127] Nicholls, C.; Karim, K.; Piletsky, S.; Saini, S.; Setford, S. Displacement imprinted polymer receptor analysis (DIPRA) for chlorophenolic contaminants in drinking water and packaging materials. Biosensors and Bioelectronics. 2006, 21, 1171-1177.

[128] Li, C.,; Ngai, M. H.; Reddy, K. K.; Leong, S. C. Y.; Tong, Y. W.; Chai, C. L. L. A fluorescence-displacement assay using molecularly imprinted polymers for the visual, rapid, and sensitive detection of the algal metabolites, geosim and 2-methylisoborneol. Analytica Chimica Acta. 2019, 1066, 121-130.

[129] Yarman, A.; Scheller, F. W. How reliable is the electrochemical readout of MIP sensors? Sensors. 2020, 20(9), 2677. 


\section{Chapter 2}




\section{Review paper preface:}

Over the decades since the first MIP was reported, the field has seen an ever increasing amount of new knowledge being brought forward and propossed. This knowledge has new and innovative methods of synthesizing and applying these polymeric receptors, leadings to a wealth of untapped information. As with all research areas, this information has to be reviewed and key areas identified that can be built upon and selected for further development. Therefore, the following chapter will endevour to search current litteriture for developments in the area of low-cost MIP-based sesnors and assay formats. These being of key interest as to understand the state of the field and to find a niche that can be developed on. Identifying sensory platforms that can be adapted and manipulated to new compounds of interest is only one aspect that has to be considered. As with all modern technologies there is the burning question of commercialization, and of recent there are very little commercial applications of MIPs. Some would argue the commercialization of technologies is what drives their development in the first place, but for the initial commercialization there has to have been a sufficient amount of research and development already conducted. The review therefore also highlights some of the key drawbacks that are preventing current MIP-based platforms from making that crucial jump from research to commercial application, alongside highlighting the new developments behind the discussed platforms. 


\section{Review paper: MIPs for Commercial Application in Low-cost Sensors and Assays - an Overview of the Current Status Quo}

Joseph W Lowdon ${ }^{1}$, Hanne Diliën ${ }^{1}$, Pankaj Singla ${ }^{2}$, Marloes Peeters

2, Thomas J. Cleij ${ }^{1}$, Bart van Grinsven ${ }^{1}$, Kasper Eersels ${ }^{1}$

${ }^{1}$ Sensor Engineering Department, Faculty of Science and Engineering, Maastricht University, P.O. Box 616, 6200 MD Maastricht, The Netherlands.

${ }^{2}$ Newcastle University, School of Engineering, Merz Court, Newcastle Upon Tyne NE1 7RU,

United Kingdom 


\begin{abstract}
:
In recent times, there has been increasing demand for point of care (PoC) analytical devices that offer users easy and rapid confirmation of the presence of an analyte of interest. The most famous and widely used examples for homebased PoC testing are the pregnancy test and the glucose meter, invented in the 1960s and commercialized in the 1970s. Since then, lateral flow assays and strip-based electrochemical devices have become the basis of handheld sensors and tests that entered the commercial market. Although antibodies and other bioreceptors offer the inherent benefit of highly specific interaction with their target, the increasing demand of cheaper, more robust receptors has led to the exploration of alternative molecular recognition mechanisms and technologies. Molecular imprinted polymers (MIPs) have emerged over the past few decades as interesting synthetic alternatives due to their long-term chemical and physical stability and low-cost synthesis procedure. They have been integrated into many sensing platforms and assay formats for the detection of various targets, ranging from small molecules to macromolecular entities such as pathogens and whole cells. In this review, we zoom in on the commercial potential of MIP technology and aim to summarize the latest developments in their commercialization and integration into sensors and assays with high commercial potential.
\end{abstract}

Keywords: Molecularly imprinted polymers, diagnostics, biosensing, lateral flow assays, commercialization 


\section{Introduction}

\section{Introduction to Molecularly Imprinted Polymers}

The field of point-of-care (PoC) diagnostics has been rapidly developed over the past few decades by the introduction of novel sensing technologies and arrays such as the home-pregnancy test or the portable glucose sensors for diabetes patients [1, 2]. The core component of these novel technologies consists of bioreceptors that drive the selective recognition of the target-ofinterest. Although bioreceptors are very powerful recognition elements due to the high affinity they inherently display for their target, their limited stability and relatively high-cost synthesis procedure have resulted in an increased research focus on the development of synthetic alternatives.

A kin to enzymes and antibodies, molecularly imprinted polymers (MIPs), might provide an answer. MIPs are artificial highly crosslinked polymeric receptors that are engineered towards the binding of specific analytes. This binding interaction is facilitated by nanocavities that are disturbed throughout the synthesized polymeric network, reflecting the conformation and chemical functionalities of the imprinted molecule or species [3-6]. The crosslinked nature of the polymer gives rise to distinct physical advantages over their biological counterparts. Enabling MIPs to resist harsh physical conditions (extreme $\mathrm{pH}$ and temperatures) that would render biological receptors useless [7-9]. This has led to MIPs being deployed into numerous fields, including chemical separation, chromatography, affinity materials for sensors, binding assays, catalysis, and sorbents for solid phase extraction, to name just a few [10-17].

\section{A brief history of MIPs}

The concept of molecularly imprinting can be dated back as far as the 1930's, with the basic principal involving the formation of a polymeric network around a small extractable template molecule to leave a complementary nanocavity was noted [18-20]. Most commonly, the imprinting process is conducted by introducing the template molecule to functional monomer and functional crosslinking agent, before initiating polymerization by the addition of a photo/thermal initiator and the requisite conditions [21-24]. The template can then be extracted by means of mechanical grinding and solid phase extraction by prolonged washing with protic solvents [25-27]. (Figure 1) 


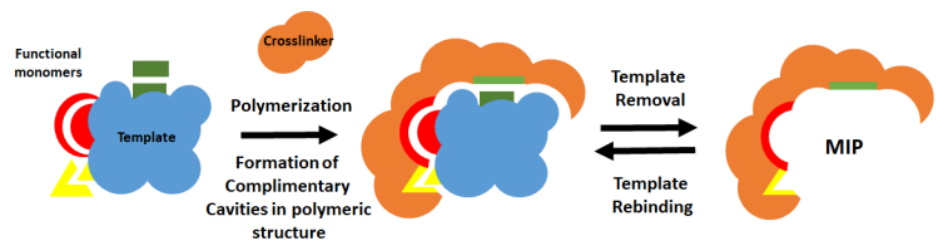

Figure 1: A generic schematic demonstrating the fundamental principles behind the synthesis and extraction of a MIP.

Altering reagent ratios, monomer and crosslinker chemical compositions, and method of polymerization enables MIPs to be tailored towards a desired application. Particle sizes tend to be the driving force behind the selection of these parameters, with smaller particle sizes offering enhanced surface area and potentially greater binding properties. Over the years, polymerization techniques have become more sophisticated with methods such as bulk and suspension polymerization offering particle sizes ranging mid to low microns [28-30], whereas micro emulsions and precipitation polymerization offer low micron to sub-micron particle sizes [31-33]. These methods were developed throughout the late $20^{\text {th }}$ century, with smaller particle sizes becoming more attractive towards the turn of the century. With smaller nanoparticle sizes offering a greater array of applications, advancing the synthesis of MIPs further. The development of solid phase synthesis ushered in the capability of reliably reproducing particle sizes in the nanometer range, offering the first reliable method of producing consistently performing MIPs [34-36].

Homogeneity in particle sizes are not the only benefits to gain from the advancements made in the field, with the imprinting process itself also being improved. Traditionally small molecules were the main target for MIPs, proving easy to imprint due to their relatively meager size. The imprinting of large molecule such as proteins, fatty residues and even cells was deemed less efficient and complex. Therefore leaving MIPs developed for the sensing of these molecules relatively unexplored. This however changed with the introduction of solid phase synthesis, as the target molecule was now immobilized on a substrate rather than dissolved or dispersed in solution [37, 38]. This immobilization process therefore opened the door for the imprinting of larger molecules, with key epitopes of the aforementioned targets now able to be imprinted [39]. This revelation allowed the field to develop further, adding the capabilities of MIPs to sense complex biomarkers (e.g. cancer cells) and offer the potential of sensing more diseases [40, 41]. 


\section{MIP technology for chemical sensing}

The idea of integrating MIPs as selective recognition layers in sensing devices and assays emerged in the 90s, coupling MIPs to traditional biosensor readout technologies that act as transducers translating molecular binding into a measurable signal $[42,43]$. Advancements in MIP synthesis procedures, microelectronics and computation have accelerated this evolution over the past twenty years, with many new sensor and assay platforms emerging [44-46]. A variety of approaches ensued, with readout methods including but not limited to quartz crystal microbalances (QCM), electrochemical sensors (e.g. capacitance, resistance, conductivity, potential difference, etc.), and thermal methods ( e.g. the so called "heat transfer method") [47-53]. The combination of MIPs and their associated readout platforms has facilitated the analysis of many different mediums (e.g. urine, blood, wastewater, soil samples, etc.) for a whole host of analytes [54-59]. Device sensitivity and limit of detection (LoD) is therefore situationally dependent on the technique and MIP employed. This deviation in sensing capabilities has meant not one MIP readout combination has been elected superior, with certain techniques favored in specific situations [60-63]. Despite the fact that all MIP-based platforms offer the advantage of low-cost, straightforward receptor synthesis and increased physical and chemical stability, commercialization seems to remain tricky.

Part of the complexity of commercializing MIP-based sensors might lie in the combination of mass producing reproducible polymer batches and integrating them into handheld readout devices. However, assays can offer an alternative to the latter. Rather than coupling a MIP receptor layer to a transducer, a MIP can be further modified to exhibit effects that can be observed by the naked eye. A diverse array of systems have achieved this ability using fluorophores, dye molecules and nanoparticles to create visually identifiable assays for a host of molecules [64-66]. This version of MIP based analysis is aimed towards semiquantitative / qualitative analysis, offering the capabilities of in the fieldtesting.

Although MIP-based assays have shown to be competitive in terms of specificity and sensitivity with state-of-the-art competitive assays such as ELISA and are compatible with e.g. lateral flow lab-on-a-chip technology, the commercial potential of these assays is yet to be fully exploited. In this review, we want to highlight the latest advances in MIP-based sensor and assay development and focus on commercially interesting platforms. We want to focus on the bottlenecks in translating commercially viable research outcomes to market-ready products and illustrate how the emergence of commercial enterprises that focus on mass-producing reproducible, highly selective MIP 
batches can accelerate the development of commercial MIP-based biosensors and assays in coming decades.

\section{MIPs are receptors in biosensors}

As previously mentioned, MIPs can be combined with a wide variety of labelfree readout techniques to create sensor devices for the detection of an essentially unlimited number of analytes-of-interest. This has led to nearly three decades of academic and applied research on incorporating MIPs into sensor prototypes. However, not all of these methods prove currently commercially viable due to several potential bottlenecks: the research is not advanced enough, the method lacks reproducibility, or the cost of commercialization is not feasible. In this article, we will summarize the most recent advances in MIP-based sensing and focus on technologies with a large potential commercial impact.

\section{Electrochemical readout}

The first fully integrated biosensor device was invented by Clark and Lyons in 1962 and has evolved into one of the most striking success stories in the history of biosensing. Glucose sensors have evolved from enzyme-modified Clark electrodes for amperometric glucose detection to wearable glucose meters combined with continuous insulin administration that have drastically changed the life of diabetes patients [67]. This evolution has led to a lot of research focus on integrating MIPs into electrochemical sensor platforms based on e.g. field-effect transistors, chemiresistors or amperometric, voltammetric, impedimetric, capacitive and conductometric readout principles [68]. In order to combine MIPs with electrochemical readouts the polymers need to have conductive capabilities themselves, or be attached to a conductive reporting layer. Carbon nano-tubes, conductive polymers (e.g. polypyrrole, polydopamine, polyaniline), and metallic nanoparticles are among the accepted methods of incorporating this trait [69]. Electrochemical MIP-sensors are extremely powerful and have shown to be capable of analyzing samples in all phases, though gaseous and liquid samples are preferable [70].

\section{Conductometric and impedimetric sensing}

Conductometric measurements rely on the time dependent monitoring of the conductivity of the MIP receptor layer, with the binding of a target analyte changing the concentration of ionic species at the solid liquid-interface [71]. Conductance measurements are relatively straightforward to perform and require little instrumentation, making the technology interesting from a 
commercial point-of-view. However, to date only a limit number have of MIPbased conductometric sensors have been developed [72-74]. Exploiting the change in ionic concentration of solid-liquid interfaces is usually done by electrochemical impedance spectroscopy (EIS). EIS analyzes the impedance of a system in dependence of an $\mathrm{AC}$, analyzing capacitive and inductive effects in addition to the purely resistive system, DC system used in conductometric sensing [75]. This makes EIS a powerful analysis tool, which has been extensively studied as a transducer mechanism in MIP-based sensing [76-78]. The compatibility with wire-based sensing and miniaturization using microelectronics makes EIS useful for PoC in vivo diagnostics, a characteristic that was exploited by Wackers et al. for the monitoring of histamine concentration in intestinal fluids. They coated titanium electrode wires in a catheter with an adhesive polystyrene layer onto which they immobilized both MIP and non-imprinted particles by dip coating in order to obtain a differential measurement setup (see Figure 2) [79]. The resulting sensor was able to detect histamine in intestinal fluid in physiologically relevant concentrations, illustrating the potential of MIP-based sensing for in vivo monitoring gastrointestinal disease markers.

MIPs have also been extensively studied as receptors for in vitro diagnostics with an interesting breakthrough being recently reported by Cai et al. They coated arrays of carbon-nanotube tips with MIP layers for the detection of papillomavirus-derived E7 protein [80]. Although the research is still somewhat on the fundamental side, the ultra-low detection limit in the subpicogram per liter range, makes the application commercially intersting in terms of diagnostics but also as a tool to monitor and study infectious diseases, a field that is highly relevant within a post-pandemic society. 


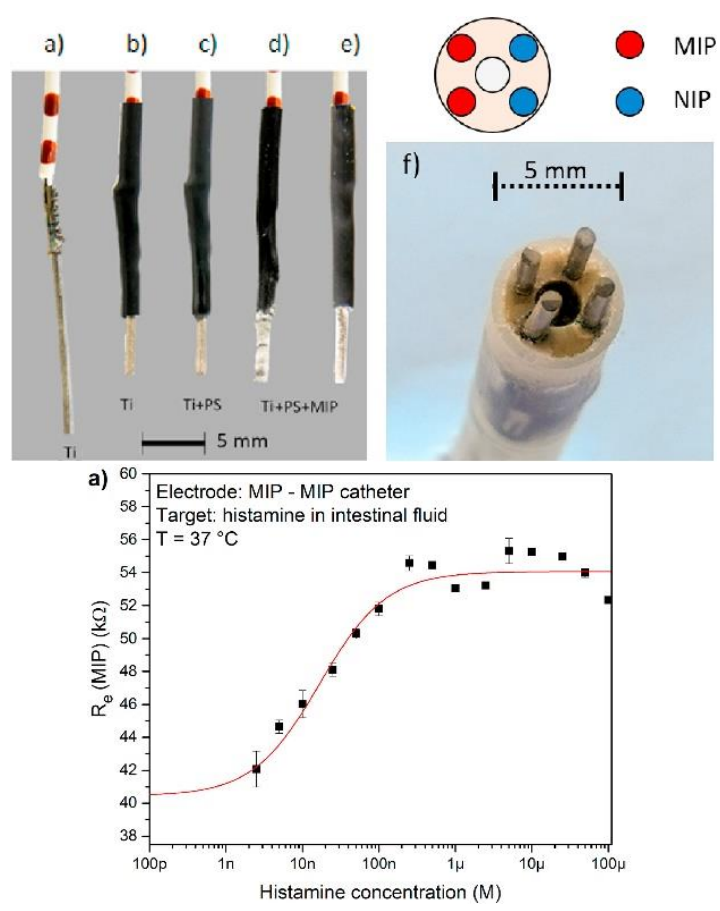

Figure 2: Thermocouples were coated with MIP particles by dip-coating a polystyrene layer onto a copper-welded titanium catheter wire (left). Two wires were coated with MIP particles, two with NIP particles and integrated into one catheter (middle), allowing for the impedimetric detection of histamine in intestinal fluid (right). Figure reproduced from [79] with permission. Copyright Elsevier 2020.

\section{Voltammetry and amperometry}

Voltammetric approaches are highly interesting for bio(mimetic) sensing due to their versatility and low noise, enabling sensor engineers to develop devices with high sensitivities [81]. These methods typically make use of three electrodes (working, counter and reference) with an aqueous electrolyte inside a defined electrochemical cell. The voltage is swept within a defined range and the resulting current is monitored in time in response to increasing concentrations of the analyte of interest. An interesting characteristic of the method is that it enables the simultaneous detection of multiple analytes (if they give rise to current peaks at different voltages). Other advantages of voltammetric sensing are the compatibility with aqueous biological samples 
and the fact that the electrodes do not have to be noble metals with many applications relying on e.g. carbon-based electrodes [82-84].

Electrochemical interferences can be suppressed by methods such as differential pulse voltammetry (DPV) and square wave voltammetry (SWV), while also increasing the sensitivity towards the desired analyte. Zhang et al. illustrated the potential of DPV for in vivo monitoring of lactate levels in sweat, an application that can be useful for e.g. monitoring athletes during training and studying their performance [85]. They managed to immobilize MIP recognition layers on a mesh of silver nanowires in a wearable patch by electropolymerization (see Figure 3). The patch, consisting of the electrodes and a miniature PDMS flow cell were connected to a DPV analyzer and allowed the researchers to analyze the lactate levels of a test person during exercise in clinically relevant concentrations (higher $\mathrm{nM}$ range). In this way, the sensor has demonstrated to be competitive with biosensor platforms and diagnostic techniques that require sampling and analysing lactate concentration in vitro. This work demonstrates that MIPs are compatible with the trend of wearable detectors for personalized medicine. Other voltammetric MIP sensors have been developed in recent years with high commercial potential for application in various fields including cancer diagnostics [86], soil contamination screening [87], water monitoring [88], and food safety [89].

Amperometric methods are a subset of voltammetric approaches that operate at a fixed, optimal potential. The analyte becomes trapped by a MIP and is reduced or oxidized at the operating potential, generating a current that is in proportion to the concentration of the analyte of interest [90]. Amperometric sensors have been the basis of most commercial glucose meters due to the combination of the advantages of voltammetry with the simplicity of working at a fixed potential, allowing miniaturization and straightforward signal processing. The incorporation of amperometric platforms have been studied almost immediately after the early start of the focus on MIP-based sensing in the mid-90s leading to many interesting detection platforms in various fields [91-93]. In a more recent study, Yang et al. imprinted poly(acrylonitrile-coacrylic acid) layers with bisphenol A. They demonstrated the detection of the endocrine disruptor in seawater and paper cups with sensitivities comparable to LC/MS/MS [94]. Other amperometric MIP sensors have demonstrated potential for the detection of prostate cancer markers [95], d-mannitol [96], and antibiotics [97]. 
1) $\mathrm{A}$
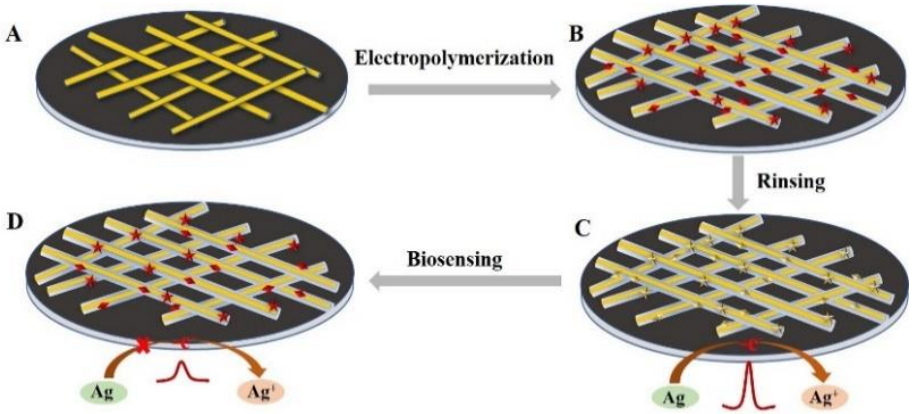

2)

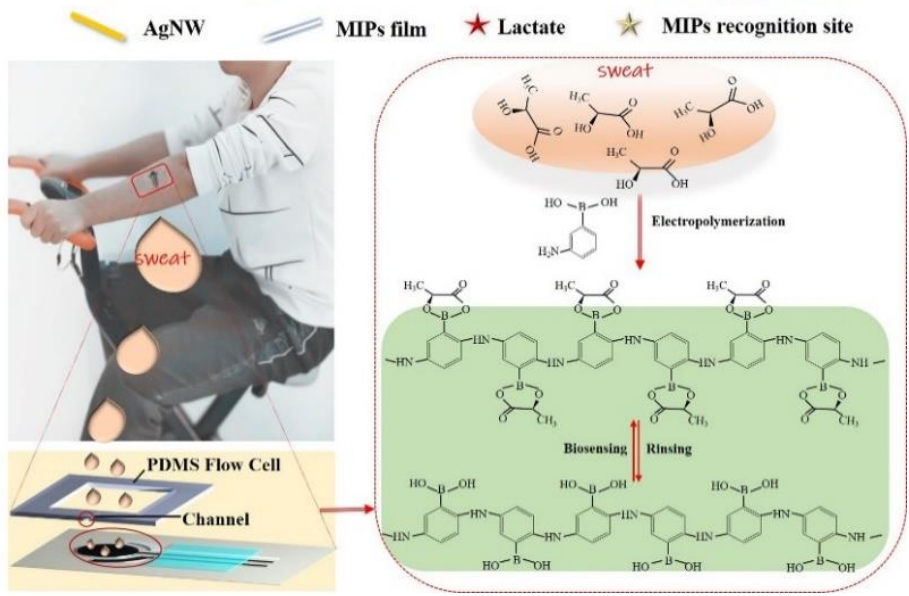

Figure 3: Lactate MIPs were made on top of a patch containing silver nanowires by electropolymerization (top). This patch, containing an integrated PDMS flow cell and a measuring chamber were attached to a test person's arm, allowing to monitor lactate levels in sweat. Reproduced with permission from [85]. Copyright Elsevier 2020.

\section{Field-effect transistors}

Field-effect biosensors typically consist of a semiconductor path separated by two conducting electrodes, the drain and the source. A third electrode, the gate, is used to apply a bios voltage over the sensor. The receptor layer is usually attached to an insulating layer (polymer, metal oxide...) covering the semiconductor path between the source and drain. Capture of the target by the recognition layer, will change the charge density on the solid-liquid interface, thereby directly influencing the conduction path between drain and source that can be electrically monitored [98]. The method offers a highly sensitive 
detection platform that was utilized by Dabrwoski et al. for the detection of human serum albumin (HSA), a marker for liver and kidney disease [99]. Bithiophene MIPs were created on $\mathrm{SiO}_{2}$ nanoparticles by electropolymerization. The FET-chemosensor was able to detect HSA in subclinical femtomolar levels, providing a non-invasive alternative for the current gold standard test that requires the drawing of blood from patients. An albumin test is rarely done in isolation and usually part of a larger investigation of a patient blood study, making a test for albumin not commercially interesting on its own. However, the study does provide an excellent proof-of-principle for the detection of proteins in blood and urine and multiplexing the platform might provide a commercially interesting alternative for traditional lab tests. The same group has demonstrated similar results, using the sensor for the detection of human chorionic gonadotropin hormone, illustrating its potential application in home-pregnancy testing, and d-arabitol for fungal infection diagnosis [100, 101]. Other groups have used FET-based MIP sensors for the detection of prostate specific antigen in human plasma [102], glucose in buffer [103], and gluten in food products [104].

\section{Acoustic wave devices}

Acoustic wave sensors utilize piezoelectric materials, typically quartz, to generate an acoustic wave over the surface of a functionalized surface. An acoustic wave vibrating at a resonance frequency is created by making use of an appropriate electrical field over the sensor substrate. Depending on the cut of the piezoelectric material, the wave can travel along the surface of the sensor (surface acoustic wave or SAW devices) or away from the surface to the bulk of the substrate (bulk acoustic wave or BAW devices) [105].

Sensors based on SAW devices typically analyze the propagation of a wave over the surface of a piezoelectric film between two interdigitated transducers. SAW resonators for MIP-based sensing have been reported on in a limited amount of publications throughout the years [106-109]. In 2017, Mazouz et al. combined imprinted polypyrrole layers with SAW technology for the ultrasensitive detection of glyphosate [110]. They managed to detect the herbicide in concentrations down to the picomolar concentration regime with limited cross-selectivity observed towards common competitors, illustrating the potential of the sensor to address a present-day hot topic in environmental monitoring. Earlier this year, Kidakova et al. reported on a similar device [111]. They immobilized protein markers for neurological diseases onto gold SAW sensor chips and electropolymerized a layer of $\mathrm{m}$-phenylenediamine to create a mold of the protein on top of the sensor substrate. Shear acoustic waves were generated by a radio-frequency signal and the observed phase shift was 
observed to analyze mass loading on the sensor surface. Although the study was conducted in buffer solution, the results are highly promising from a diagnostic point-of-view, illustrating that the sensor is capable of distinguishing between cerebral dopamine neurotrophic factor and its homologue at concentrations in the sub-pg/mL range. This indicates that the sensor is competitive with state-of-the-art ELISA platforms and is able to detect protein markers within or even below the clinically relevant concentration regime.

The amount of BAW devices for MIP-based sensing that have been developed heavily outweighs the number of MIP sensors based on SAW technology. This finding can mainly be attributed to the popularity of quartz crystal microbolances (QCM) that have formed the basis of hundreds of publications on chemical sensing in the past twenty years [112-114]. A QCM utilizes a slim piezoelectric quartz crystal that is sandwiched between two circular metal electrodes, which vibrates at a resonance frequency by applying an alternating current field. As the mass on the surface increases $(\Delta m)(\mathrm{ng})$, a proportional change in resonance frequency $(\Delta f)(\mathrm{Hz})$ can be observed, as described by the Sauerbrey equation (mass sensitivity constant (C) $\left.\left(\mathrm{ng} \mathrm{cm}^{-2} \mathrm{~Hz}^{-1}\right)\right)$ [115];

$\Delta m=-C \Delta f$

MIPs are coated onto the surface of the crystal offering the possibility of realtime measurements in both the gaseous and liquid phases [116-119]. When a binding event occurs between the MIP and the target molecule, the change in mass at the surface causes a shift in the resonance frequency. This makes QCMbased sensors particularly interesting for detecting macromolecular targets such as proteins [120-122], viruses [123-125], bacteria [126-128], or whole cells $[129,130]$.

In 2018 Battal et al. developed a sensor for the detection of synthetic cannabinoids [131]. The QCM-based sensor displayed excellent selectivity for several commonly encountered cannabinoids and was able to detect them in spiked synthetic urine samples in concentrations down to the sub-pg/mL concentration regime (Figure 4). The sensor is commercially very interesting as it can find applications in various fields including clinical toxicology, forensic research and law enforcement, as these very addictive, potentially lifethreatening designer drugs are flooding both the illicit and legal drug market [132]. Additionally, they are hard to detect using current drug tests, as drug producers tend to change their structure rapidly to avoid prosecution. 

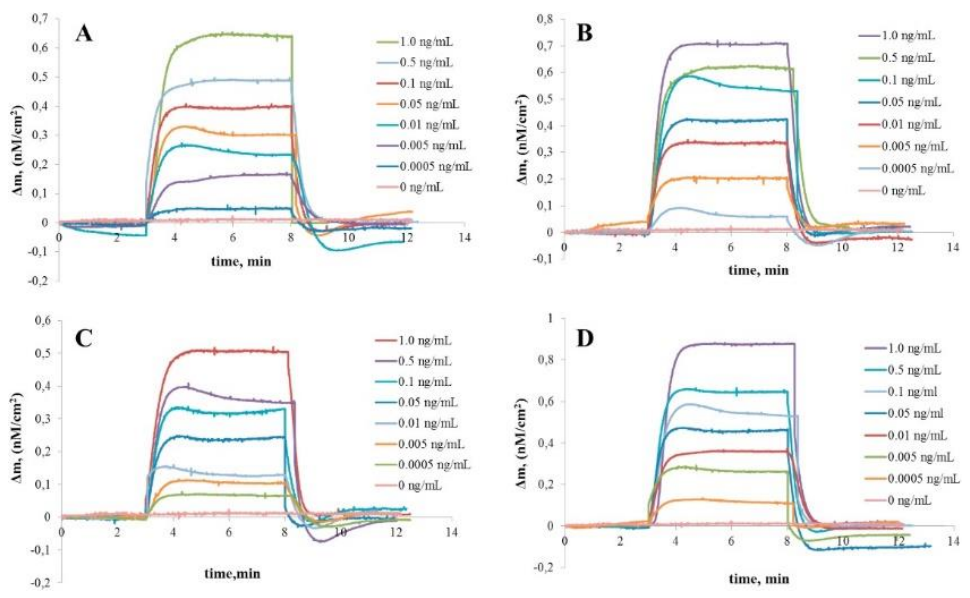

Figure 4 QCM-based detection of 4 different cannabinoids (JWH-018, JWH-073, JWH-018 pentanoic acid and JWH-073 butanoic acid respectively A-D). The data show that it is possible to quantify these compounds in synthetic urine down to concentrations in the pg/mL range. Re-used from [131] with permission. Copyright Elsevier 2018.

MIP sensors based on QCM technology also have potential in personalized medicine as evidenced by a publication of Chunta et al. in 2019 [133]. They constructed a MIP sensor capable of detecting high-density lipoprotein (HDL) cholesterol in the clinically relevant concentration regime. Their sensing platform showed little to no cross-selectivity towards other (lipo)proteins in blood and displayed and excellent correlation with the colorimetric enzymatic test that is considered to be the golden standard. In addition, the sensor does not require sample pre-treatment and is therefore a lot faster than the latter. The authors then integrated this sensor into an array along with their previously developed sensor for low-density lipoproteins (LDL) to deliver a proof-of-concept for a multiple analyte self-test for metabolic syndrome [133, 134]. Other interesting sensor platforms have recently been developed for application in food safety [135-138], environmental screening [139-141], and pathogen detection $[142,143]$.

\section{Thermal readout}

The development of thermal methods that incorporate MIPs is a relatively untapped resource, and only over the last decade, the prospect of a thermal read-out has begun to be explored [144]. Initial publications exploring MIPbased thermal methods are spars, with the first thermal MIP sensor with high 
application potential outlined by Lettau et al. [145]. This paper outlined how phenylacetate could be catalytically converted using a column packed with MIPs, leading to an increased temperature observed in the collected filtrate from a flow reactor. A follow up to this work introduced the first MIP-based thermistor in organic solvent, with binding events to the MIP observed as an exothermic peak signal that appears after the injection of an analyte. The paper then further elaborated on the differences that could be witnessed between the MIP and NIP, generating thermal readings in a similar manner seen to a traditional batch rebinding experiment. Similar platforms have been developed by the same group over the years [146-148].

A more direct thermal method emerged in 2012 when van Grinsven et al. reported on a novel thermal method for the identification of DNA point mutations using DNA melting curve analysis [149]. It was discovered as the temperature of DNA increases it undergoes a defined conformational change, increasing the surface area of itself by nearly $150 \%$. This observation was known to increase the electrical resistance in the system, but the increase in thermal resistance was unreported. The readout technology was soon combined with MIPs for the detection of low-molecular weight compounds [150-153] as well as macromolecular targets including proteins [154], mammalian cells [155-157], and microorganisms [158-160] in various matrices including wastewater, urine and blood.

The method controls the input temperature underneath the sample in a stringent manner, while passively monitoring the temperature in the liquid measuring chamber above the sample passively in time. Binding of the target to the solid-liquid interface leads to an increase of the thermal resistance, which can be measured as either a decrease of the temperature inside the flow cell (constant input temperature) or a phase shift on the transmitted thermal wave [161]. The method offers a very low-cost readout platform, which requires little instrumentation, but the sensitivity of the system is restricted. The main problem associated with this technology is producing the receptor layers, with imprinting tricky to standardize in terms of mass-production and receptor immobilization.

In 2017, the technology was taken one step further towards commercialization by coating thermocouples with bulk imprinted MIP particles imprinted with cortisol and serotonin (see Figure 5) [162]. An adhesive layer was immobilized on the thermocouple and MIP particles were attached to the layer using dipcoating, enabling to directly detect neurotransmitters in liquid samples without the need for planar functionalized substrates. These findings open up the possibility of integrating thermocouples into $e . g$. catheters for the direct in 
vivo detection of small molecules. More recent research, focused on improving the sensitivity of the technology by directly grafting MIP receptor layers on planar electrodes for the detection of designer drug molecules [163]. Last year, Cornelis et al. reported on a new update from the readout technology side; they improved the detection limit of the methodology by two orders of magnitude by using a printed meander-structure as a heater and sensing element directly underneath the receptor layer [164]. The temperature inside the flow cell was monitored by a thermopile, enabling them to detect $E$. coli in apple juice in concentrations down to $100 \mathrm{CFU} / \mathrm{mL}$.
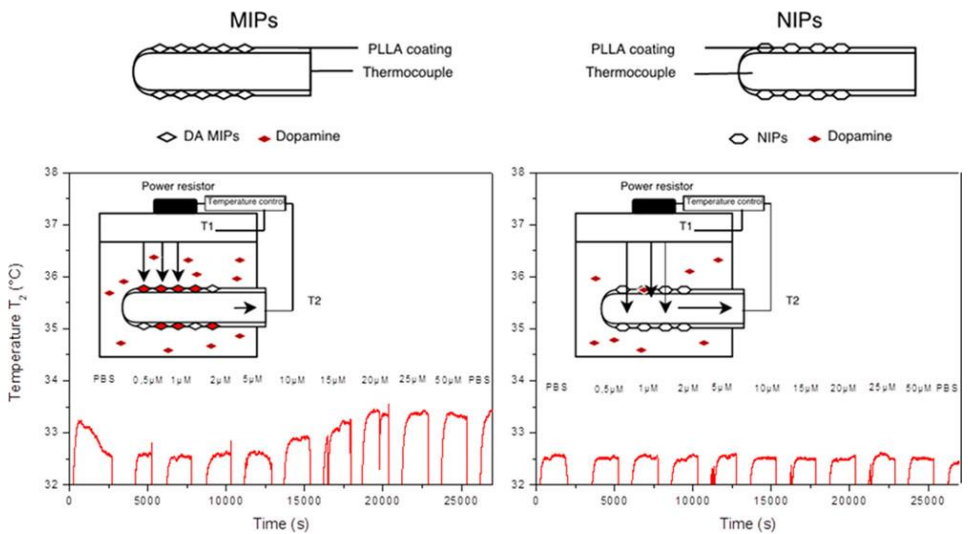

Figure 5 Thermocouples were dip-coated with a poly-lactic acid adhesive layer and rollcoated with MIP particles. These thermocouples were used to detect the presence of various neurotransmitter in buffer. Re-used from [162] with permission on an open-access license agreement, Copyright American Chemical Society 2017.

\section{Optical sensors}

Optical sensing principles have been extensively used as transducers in a wide variety of MIP-based sensing devices and assays [165-167]. This group of detection platforms is quite heterogeneous and it is often hard to mark the border between sensors and assays. An example operating at the border of both would be a lateral flow assay connected to a surface plasmon resonance (SPR) analyzer. For the sake of simplicity, we will only discuss the direct detection of analytes on a sensor surface in this chapter, while all platforms that are based on luminescence, fluorescence or fluorescence quenching, dye or label displacement, enzymatic, pseudo-enzymatic or non-enzymatic color reactions and similar principles will be discussed in the next chapter as part of MIP-based assays. 
SPR can be considered as one of the most powerful, and therefore popular, readout technologies and has been combined with MIPs for the detection of microorganisms [168, 169], amino acids, peptides and proteins [170-172], dangerous explosives [173-175], antibiotics [176-178] and other low-molecular weight compounds [179-183]. Detection upon rebinding of the target is based on changes in electron density at the surface of the sensor chip. Ertürk Bergdahl et al. reported on the development of a biosensor with high diagnostic potential last year [184]. They used microcontact printing to imprint tyramine layers on gold electrodes with secreted bacterial factor (RoxP). In this way, they managed to detect RoxP in sub-nanomolar concentrations with limited interference from other proteins. They used this sensor to detect RoxP in skin swabs (Figure 6) and validated the results using ELISA, thereby highlighting the potential applicability of the sensor in diagnostics. Other recently developed SPR-MIP sensors were used for the detection of anticancer drugs [185] and cardiac biomarkers [186].

Surface-enhanced Raman Spectroscopy (SERS) is another tool that has been used as an optical transducer for the direct detection of target-MIP rebinding at solid-liquid interfaces $[187,188]$. SERS is particularly interesting because of its ultra-sensitive detection mechanism that allows for the detection of analytes in the femtomolar range [189]. Interesting applications using SERS-based sensors have been developed in recent years mainly focusing on food safety and environmental monitoring [190-193]. 


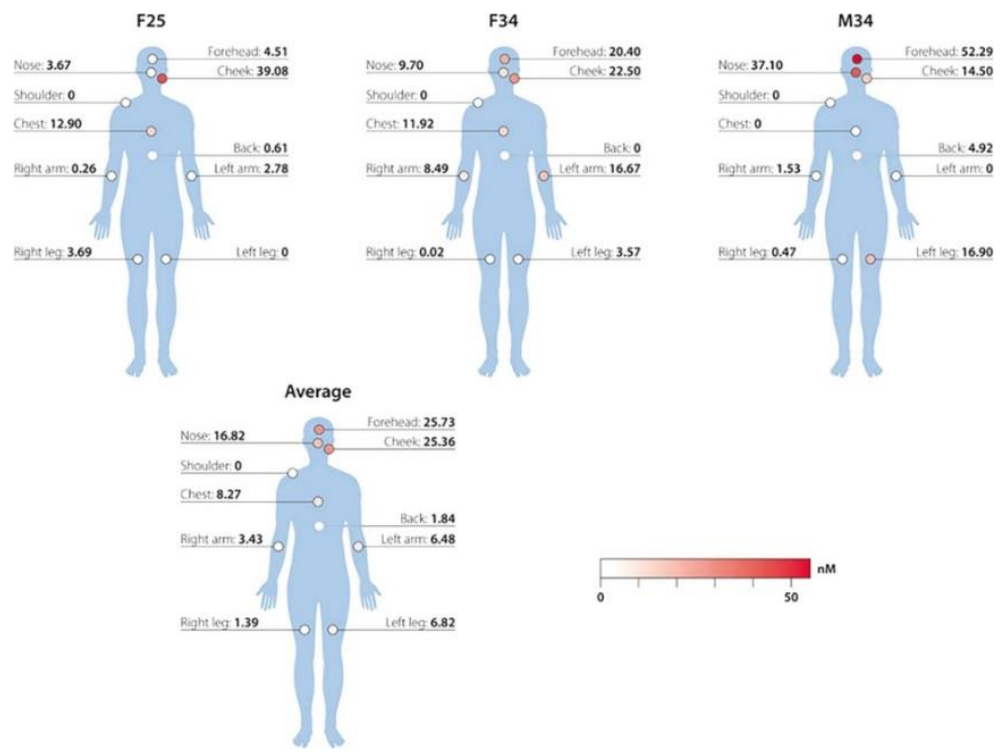

Figure 6: Gold SPR chips were coated with MIPs for the quantification of secreted bacterial factor (RoxP). Skin swabs were taken at various positions on the bodies of two female and one male volunteer and the MIP-based SPR sensor was able to detect RoxP on all positions with results being validated using a golden standard ELISA test. Adapted from [184] with permission. Copyright American Chemical Society 2019.

\section{Bottlenecks commercialization MIP sensors}

Despite tremendous advances in both imprinting technology and micro- and nano-electronics, commercially interesting MIP-based sensor platforms have only been reported on in a research setting. The valorization process, translating lab-based technology to the market is complex and in addition to commercial bottlenecks, which will not be studied in detail in this manuscript, many technical challenges need to be overcome to turn MIP sensors into true competitors for commercial biosensors, molecular diagnostics and traditional lab analysis.

From the device side, technical issues can lay either in creating handheld or user-friendly portable systems or in the creation of disposable, functionalized electrodes. The former is illustrated by the fact that handheld portable glucose meters are usually based on a straightforward conductiometric readout platform that allows for easy miniaturization and relatively simple calibration. 
However, physiological glucose levels are typically in the millimolar range and creating sensors for other compounds might require higher sensitivities that require other readout principles that operate in the (sub-)nanomolar regime. With the field of micro- and nano-electronics rapidly expanding, commercial enterprises are coming up with simplified handheld/portable solutions: Palmsens offers portable alternatives for the high-end impedance analyzers by $\mathrm{HP}$ or Zürich Instruments for lab analysis. Similar trends can be observed for e.g. QCMs and SPRs with companies like Aspectus and OWLS building portable low-cost alternatives for high-end lab equipment offered by respectively QSense and Biacore (GE). As these devices will improve, the chance of companies not only offering platform technology but also integrated devices for specific biosensing applications will increase.

The authors of this paper are also working on founding a company, SENSIP $\mathrm{BV}$, based on their thermal sensing technique. Both the possibility of creating a commercial readout platform and developing specific sensor applications are being explored. Therefore, apart from miniaturizing readout design and upscaling device production, a lot of R\&D budget will be devoted to creating reproducible functionalized electrodes. In terms of particle immobilization onto wire-based or planar electrodes, research focuses on various angles including dip-coating, roll-coating, electro-polymerization, surface grafting and roll-to-roll imprint lithography. An even more challenging third bottleneck that can be identified lies in the reproducible creating of large batches of MIP particles or layers. Achieving homogeneity in both morphological characteristics as binding affinity is a complex tasks and makes it hard to get rid of inter-batch variability, which is obviously detrimental in terms of creating commercial sensor applications. 


\section{Low-cost MIP assay formats}

A possible alternative to integrated sensor devices that can address some of the proposed bottlenecks lays in the use of MIPs as recognition elements in assays. Although both terms are often used interchangeably in literature and there is some overlap between the concepts, making the difference feel somewhat arbitrary, we define MIP-based assays as sensing applications that indirectly detect target-rebinding leading to an optically obtainable signal, potentially amplified along the way. This facilitates both readout, as the result can often be seen with the naked eye or requires e.g. a smartphone camera, and receptor immobilization, as lab-on-a-chip technology allows for the creation of lateral flow assays and dipstick devices. An example from the biosensing industry can be found in home-pregnancy testing. Introduced in the 1970's, the home pregnancy test is a sandwich immunoassay that grants the colorimetric determination of the presence human chorionic gonadotropin (hCG) [194]. Comprising of a porous pad with a series of reactive components, a sample is introduced onto the surface of the pad and is carried along the device by capillary action alone. While traversing the pad the liquid sample (e.g. urine, blood, saliva) interacts with the reagents imbedded in the microstructure of the pad, in turn selectively chemically labelling the target analyte. Engineered antibodies comprise the backbone of this chemical labelling process, expressing desired physical traits (radioisotope, fluorescence, and chromophore) [195].

As with antibodies, MIPs can be manipulated and modified to exhibit specific traits upon the binding of a target analyte. MIPs therefore pose as a genuine synthetic alternative to previously biological sensing elements, with the earliest report of a "molecularly imprinted assay" being reported in 1993 by Vlatakis et al. [196]. This assay was analogous to competitive radiolabeled immunoassays, employing an imprinted ethylene dimethacrylate-comethacrylic acid polymer to sense the presence of two unrelated compounds (theophylline and diazepam). The results of this demonstration were impressive, with a linear range of $14-224 \mu \mathrm{M}$ for theophylline and the results from 32 serum tests correlated with the same analysis from a corresponding enzyme-multiplied immunoassay technique. Cross-selectivity was proven to be on par with that of antibodies, though the major pitfall came at the cost of the developed MIP-based assay operating in organic solvent. Assays capable of operating in aqueous buffer solution and organic solvents were soon introduced, paving the way forwards for MIP-based assays to operate in biologically relevant environments [197-199]. 
Though the binding of target analytes and environments analysis could be conducted had drastically been improved, the use of radiolabeling was hindering the commercialization of the generated assays. The availability, handling, cost and coupling of isotopic labels was brought into question, deeming radiolabeled assays as unfeasible. Other methods were soon developed showing greater potential, with Piletsky et al. being one of the first to introduce fluorescently labelled analogues of triazine for the detection of unlabeled triazine [200]. Fluorescent characteristics would soon be incorporated into the physical properties of the imprinted polymers, utilizing fluorescent monomers, crosslinking agents and initiators [201]. This allowed fluorescent enhancement and quenching to be considered as potential mechanisms for the direct detection of analytes. Other methods were also considered, with MIPs being conjugated to enzymes offering enhanced sensitivities. Though with the integration of biological components the environments in which analysis can be carried out, once again becomes limited. However, Literature sources report the use on enzymes in conjugation with MIPs in colorimetric and chemiluminescence methodologies [202]. Direct coupling of modified analytes with powerful chromophores such as dye molecules has also been reported, enabling visual confirmation of binding events [203].

Since the inception of these concepts and methodologies, the field has however developed further; utilizing technologies not previously available to increase the commercial potential of these strategies. These advancements are guided by the needs of both commercial and industrial applications, increasing the ease and speed of analysis. The innovation of the last decade has therefore brought these concepts closer to commercial fruition, bringing the need to reexamine the current state-of-the-art (Quantum dots, nanoparticles, displacement assays) and determine the current obstacles that must be overcome before these technologies will be translated to commercially available devices.

\section{Quantum dot MIP assays}

The use of conventional organic fluorophores in combination with imprinted systems has seen a decline over the last decade, with the use of quantum-dots (QDs) becoming ever more popular. QDs offer greater chemical stability, photostability and tunable spectral properties than traditional fluorophores, gifting distinct advantages to systems that utilize these fluorescent nanoparticles [204]. Thus QDs pose as a more capable, controlled, and reliable method of installing fluorescent characteristics into molecular imprinted systems. The first reported affiliation of QDs with MIPs was reported by Lin $e t$ 
al. in 2004 [205]. MIP layers were anchored onto CdSe/ZnS core-shell QDs permitting the selective detection of caffeine, uric acid and estriol in several solvents with the biggest effect size encountered in water, which opened up the possibility of using the system for biological analysis. This article however, only provided a first proof-of-principle and over the years, other groups were able to come up with more in-depth studies, demonstrating actual application of MIP-QD assays for e.g. environmental monitoring with LoDs reported in the relevant $\mathrm{nM}$ concentration regime [206-207].

In recent years, the amount of assays based on the combination of QDs and MIPs has rapidly increased with several commercially interesting technologies reported on by a wide range of groups. Vaneckova et al. formed thin imprinted polydopamine films with modified QD-antibody-antigen conjugates as the imprinted species for protein detection [208]. Laser ablation inductively coupled plasma mass spectrometry (LA-ICP-MS) was then utilized in this proof of principle immunoassay, undertaking a dual approach that highlighted the sensitivity (from the MIPs) and selectivity (from the QDs-antibodies) of the method. The resulting LoD of the methodology was $4.2 \mu \mathrm{g}$ and $1.6 \mu \mathrm{g}$ respectively, with the analysis time of each scan only taking 23 seconds. The main drawback of this technology is still the application of complex readout devices that increases the size and cost of the technology.

As most fluorescent systems utilize an optical readout method for interoperating the fluorescence signal, either the readout device must become compact (handheld) or the necessity of readout methods for validation must be overcome. Wang et al. overcome this necessity by introducing a facile QDMIP based method for the direct detection of dopamine in tiny amounts of biofluid [209]. The system that is introduced is a paper-based assay that allows the detection of physiologically relevant amounts of dopamine in $10 \mu \mathrm{L}$ of serum. Dual-emission (carbon and CdTe) QDs act as a core that is encapsulated inside a MIP shell, enabling spectroscopic and visual verification of the presence of dopamine in the presence of UV light. To facilitate ease of use, the nanoparticles were loaded onto small strips of filter paper by adsorption of the particles directly into the crevices of the substrate. The strips were then exposed directly to serum samples, and analyzed with a spectrometer and UV lamp (Figure 7). The dose-response curve for this system displayed a wide linear range $\left(0-1.2 \times 10^{-6} \mathrm{M}\right)$ even in the presence of common ionic and molecular interfaces that can be found in biofluids, yielding an LoD of 100-150 $x 10^{-9} \mathrm{M}$. All results were validated with HPLC, which emphasizes that the assay is not only interesting because it is handheld and requires little instrumentation but also has enough diagnostic relevance. Over the past two years alone, many similar QD-based MIP assays have been developed for 
various purposes including medical diagnostics [210-212], food safety [213214] and environmental screening [215-216].
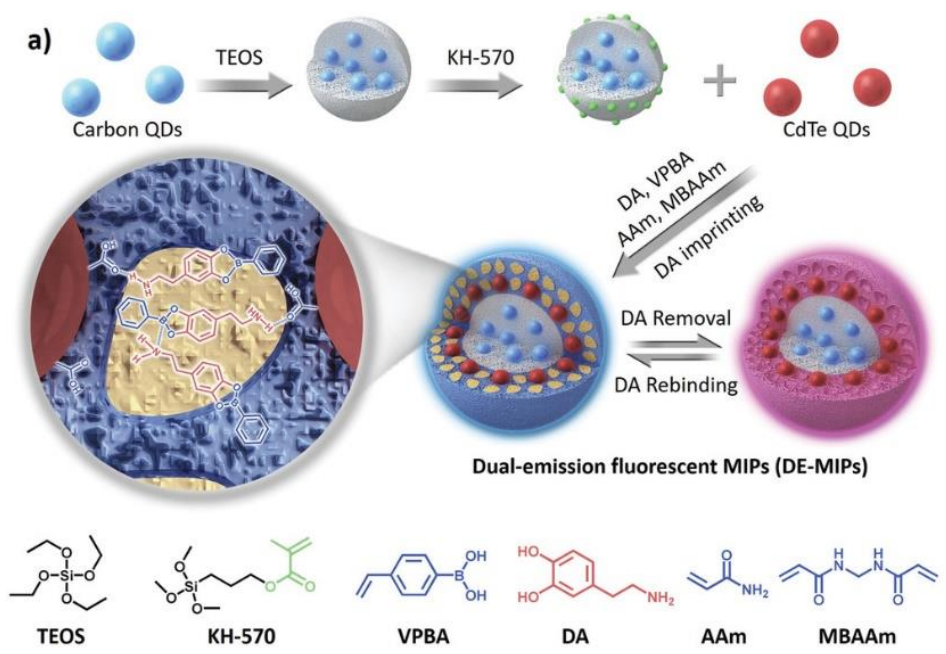

b)

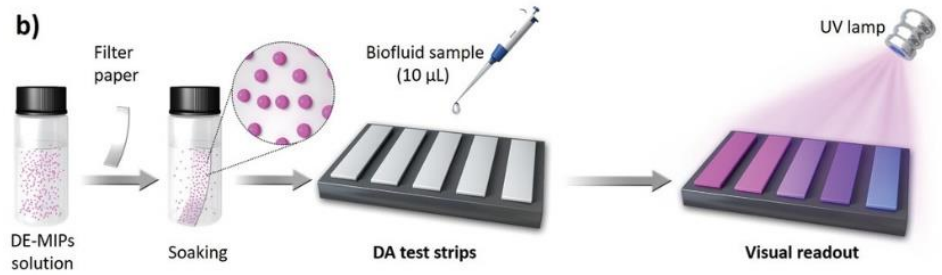

Figure 7: Fluorescent dual-emission MIP nanoparticles were made by integrating red and blue fluorescent quantum dots into the polymer shell (top). These particles were immobilized onto test strips by soaking them in MIP solution. The result is a $\mathrm{pH}$-indicatorlike test strip that allows the end-user to quantify the amount of dopamine in a biological sample. Adapted with permission from [209], Copyright John Wiley and Sons 2018.

\section{Nanoparticle MIP assays}

In addition to QDs, nanoparticles (NPs) have been used in conjunction with MIPs to create optical detection assays. Though some literature demonstrates that NPs can be incorporated into the bulk of MIPs [217], the majority of literature utilizes NPs as a separate sensing element. These assays are often combined with colorimetric detection, overcoming the need for an excitation source and fluorescent probes. 
Wu et al. reported on the use of NPs with MIPs for the colorimetric detection of residues of the insecticide Cartap in tea [218]. Cartap was extracted from spiked and non-spiked samples of tea by means of magnetic molecular imprinted microspheres ( $\left.\mathrm{Fe}_{3} \mathrm{O}_{4} @ \mathrm{mSiO}_{2} @ \mathrm{MIPs}\right)$ that utilized methacrylic acid as a functional monomer. Cartap was eluted from the MIPs and AgNPs were added to stimulate a color change turning the elution from yellow to grey dependent on the presence of the target. In this way, a semi-quantitative assay was developed allowing for the detection of Cartap in concentrations down to $\pm 5 \mathrm{mg} \mathrm{L} \mathrm{L}^{-1}$. The reaction can be further quantified using a UV-vis spectrophotometer, allowing for an LoD of $0.01 \mathrm{mg} \mathrm{L}^{-1}$. Although this method is very interesting due to its simplicity, the multistep procedure makes it timeconsuming and is not very sensitive. Zhao et al. introduced a method that can address the latter aspect, by using AuNPs for signal enhancement; they were able to construct a colorimetric sensor for the detection of atrazine with an LoD of $1.2 \mu \mathrm{g} \mathrm{L}^{-1}$ and an LoQ of $4.0 \mu \mathrm{g} \mathrm{L}^{-1}$ [219]. This makes the assay competitive with most sensor applications that are able to detect targets in the lower nanomolar concentration regime. However, sample pretreatment is still necessary for sample analysis, limiting the method's commercial potential. Other reports of nanoparticles being used in a similar fashion exist, though they all suffer the same drawbacks preventing commercial viability [220].

Kong et al., highlighting the potential of a system that does not rely on sample pretreatment, developed a novel microfluidic paper-based colorimetric sensor based on MIPs and AgNPs [221]. A system was constructed that consisted of a MIP membrane that was imprinted with bisphenol A(BPA) (Outer layer), $\mathrm{ZnFe}_{2} \mathrm{O}_{4} \mathrm{NPs}$ (second layer) and cellulose paper (Inner layer) (Figure 8). Upon the addition of 3,3',5,5'-tetramethylbenzidine (TMB) and peroxide to the structure, the NPs would oxidize the introduced peroxide generating $\mathrm{OH}$ species that would discolor the cellulose paper. The binding of BPA to the MIP layer would prevent this interaction, leaving the color of the cellulose paper unchanged.

This proved to be an extremely sensitive method with a LoD of $6.18 \mathrm{nM}$ and a linear range of $10-1000 \mathrm{nM}$. Photoshop was used to quantify the color change, relating the change in greyscale to the concentration of BPA introduced. These results prove that it is possible to develop a low-cost MIP-NPs assay that can be used for the detection of compounds in a relevant concentration regime using a simple camera and image processing software. The method does have some drawbacks such as $\mathrm{pH}$ deficiency and the need for environmentally unfriendly reagents. However, the combination of this type of assay in combination with a lateral flow device in the future could minimize the 
environmental impact of the method, as the required reagent volume would be drastically decreased.

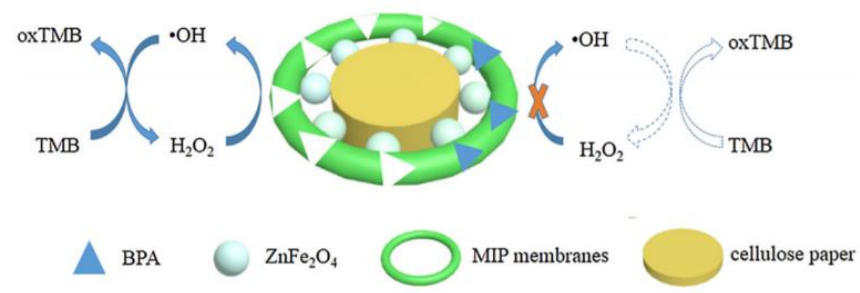

Figure 8: MIP membranes deposited round a cellulose paper, using nanoparticles as a spacer ring between the paper and the MIP layer. The MIP membranes selectively bind BPA, preventing the oxidation of peroxidation, leaving the color of the cellulose paper unchanged. In absence of BPA, the generation of $\mathrm{OH}$-species will discolor the paper. Reused with permission from [221], Copyright Elsevier 2017.

\section{Colorimetric and fluorometric MIP displacement assays}

Displacement assays act as competitive assays between a labelled version of a target analyte and a non-labelled version. The modified analogue of the target species has specific functionalities facilitating the molecule to exhibit colorimetric or fluorometric properties. The first proof-of-principle of the concept was conducted in 1998 by McNiven et al. using a dye-labeled conjugate to detect chloramphenicol [222]. The colorimetric sensor proved immune to interferences and had a linear response to the over a $3-1000 \mu \mathrm{g} \mathrm{mL}^{-1}$ range, being effective above, within and below the therapeutic range. Nicholls et al. detail a fluorescent variation of the approach in which they reported a displacement assay format for the detection of chlorophenolic contaminants in drinking water and packaging material [223]. Fluorescently labelled pentachlorophenol (PCP) was prepared as a "guest" molecule and used in a competitive binding assay format. Both labelled and non-labelled PCP was introduced in the presence of the MIP with less retention for the guest molecule observed. The guest molecule was also pre-bound to the MIP, with the reporter molecule being displaced in the presence of the higher affinity unmodified PCP analogues. The filtrates collected from the analysis were then tested with luminometers and compared to GC-ECD as a reference. A detection limit of 0.5 $\mu \mathrm{g} \mathrm{L} \mathrm{L}^{-1}$ was achieved, though in comparison to antibodies the method lacked sensitivity. These reports also focused on the spectral analysis of the method, whereas Li et al. highlighted in a more recent study how the method could be used for the visual verification of algal metabolites [224]. Though a greater sensitivity was not procured $\left(48 \mu \mathrm{g} \mathrm{L}^{-1}\right)$, visualization of the displacement of 
fluorescently labelled analogue was made possible with a hand-held UV lamp (Figure 9). Thus removing the need for large and expensive pieces of analytical equipment.

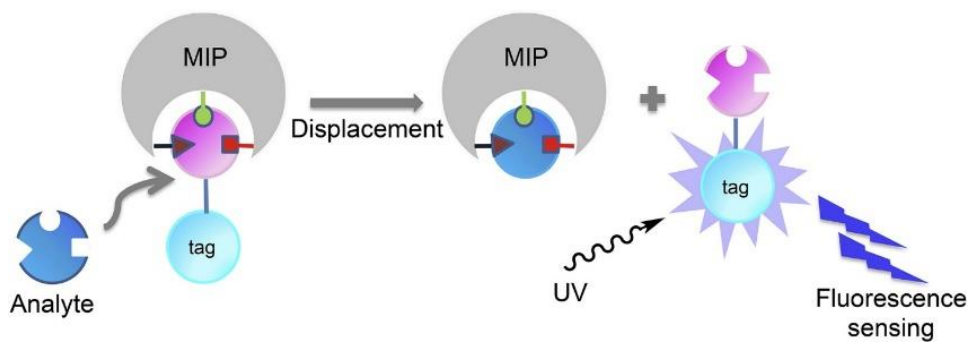

Figure 9: MIPs were imprinted with algal metabolites geosmin and 2-methylisoborneol, extracted and loaded with substrates coupled to fluorescent tag. In presence of the target, the fluorescently labeled substrate gets displaced due its lower affinity for the MIP. Illumination of the filtrate with a simple UV-lamp visually confirms the presence or absence of the target. Adapted with permission from [224]. Copyright Elsevier 2019.

One facet reducing commercial potential of MIP displacement assays is the need to include a chromophore/fluorophore. However, it has been shown that development of a reporting analogue is not required, and an unrelated (fluorescent) dye can be utilized instead. The first example of this was proposed in 1998 by Haupt et al. in which a fluorescent ligand displacement assay was outlined [225]. The assay uses a coumarin derivative as a nonrelated fluorescent probe for the detection of the herbicide 2,4-dichlorophenoxyacetic acid, producing a specific and selective probe that achieve a detection limit of $100 \mathrm{nM}$. Piletsky et al. demonstrated the same principle for the detection of Lphenylalaninamide by dye displacement [226]. Pre-loading the dye molecule onto the MIPs before loading the particles onto a column and eluting an aqueous solution containing the target molecule through. Drawing attention to the merits of high reproducibility yet maintaining a detection limit in the low $\mu \mathrm{M}$. As chromophores are easy to incorporate into the method, Greene et al. showed that visual verification of binding events was possible [227]. Benzofurazan dyes were used in a competitive binding assay in the presence of six imprinted amine compounds that could be easily identified by the naked eye without require spectroscopic method.

Several MIP-based displacement assays have been used in recent years for the detection of a wide variety of compounds including biogenic amines, 
pesticides and narcotics [228-230]. The most apparent obstacles that need to be overcome to allow for commercialization lie in improving the sensitivity of these methods and the creation of homogenous batches of MIPs with high affinity. The use of dyes with high extinction coefficients could improve the sensitivity in the future, while integrating the assays in lateral flow devices can create dipstick devices suitable for market introduction.

\section{MIPs as capture antibodies in immunoassays}

In addition to being used as antibody-analogues in competitive assays as described in the previous chapter, MIPs have also been used as capture probes in traditional ELISA(-like) pseudo-immunoassays [231-233]. These type of assays profit from the popularity and associated technological advances that have been associated with ELISA-like assays. The ubiquity of immuosorbent assays in present day diagnostics and analytical chemistry could overcome some of the thresholds associated with introducing new technologies into a well-established market. Furthermore, immuoassays are compatible with labon-a-chip technology, which opens up the possibility of integrating the assay into a lateral flow device. This characteristic was exploited by He et al. for the creation of a test strip for the pesticide triazophos (Figure 10) [234].
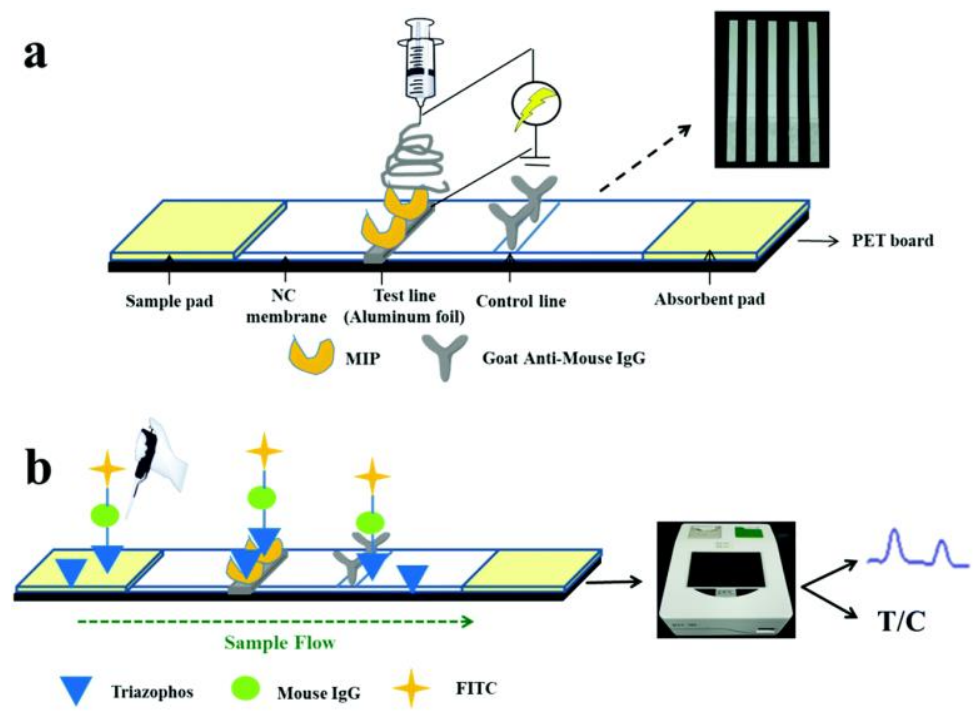

Figure 10: Triazophos MIPs can be used instead of antibodies in a lateral flow assay. MIPs were used as capture antibody in the sorbent test line path of the assay. Samples were 
mixed with FITC-labeled target molecules. Presence of the target prevents the labeled molecules to bind to the test line, leading to an inverse correlation between target concentration and fluorescence intensity on the test line. Adapted from [234] with permission. Copyright Centre National de la Recherche Scientifique (CNRS) and the Royal Society of Chemistry 2020.

They immobilized an imprinted cellulose acetate layer on the test line of a lateral flow strip. Samples travelled by capillary transport from the sample pad to the absorbent pad via the MIP layer. The sample containing an unknown concentration was mixed with a solution containing a conjugate of triazophos with an IgG and a FITC label. When the sample reaches the MIP line, the conjugate will compete with any target molecule present in the sample, resulting in an inversely proportional correlation between target concentration and the signal. The results were benchmarked with LC-MS/MS with an LoD of $20 \mu \mathrm{g} \mathrm{mL}^{-1}$ in tap water. A similar assay was developed by Akbulut and Zengin deposited imprinted poly (N-acryloyl-L-phenylalanine) films on whatman paper strips for the detection of propranolol in human plasma [235]. These portable tests themselves are suitable for on-site analysis but all results have been assembled using a lab UV-vis spectrophotometer. Additional tests in the future will have to prove that these tests can detect targets in relevant concentrations when combined with e.g. smartphone cameras, which have been used by other groups in the context of MIP-based sensing [236-237].

\section{Bottlenecks MIP-based assay platforms}

The overview presented in this chapter illustrates the commercial potential of MIP-based assays. In comparison to integrated sensor devices, receptor immobilization is often a lot easier with MIPs often simply being immersed in solution or deposited onto the bottom of a 96-well plate. In addition, assays profit from the popularity of immunoassays and can be easily integrated into lateral flow strips or dipstick, although they are also compatible with most forms of biosensors, especially those based on optical transducers. In addition, colorimetric assays can often be analyzed by the naked eye, as do some assays based on luminescence and fluorescence that do require some form of excitation source. Even the quantifiable assays that are now being analyzed using lab-based spectrophotometers could easily be transferred to portable systems that are compatible with smartphone cameras or handheld spectrophotometers.

Up until now, the major bottleneck in developing MIP-based assays for commercial use has been, again, the production of homogenous receptors with high affinity on a large scale. Enzyme-based assays and immunoassays profit from the knowledge and knowhow the industry has in mass-producing 
them, especially with reactor technology enabling upscaling the production of these bioreceptors. However, MIP synthesis is profiting from the rapidly advancing field of polymer technology and the number of groups reporting on novel ways to create MIP particles, that are more homogenous in morphology and affinity towards their target, are increasing. With the development of these new techniques, new companies focusing on large-scale MIP synthesis are entering the commercial market, as we will discuss in the next chapter.

\section{Commercial MIP synthesis}

As described in previous sections, despite the huge commercial potential of imprinting technology ( $>1000$ patents on Scifinder), until date, the amount of companies that direct their resources towards MIPs is limited and efforts in this area have mostly been confined to laboratory research. Early commercialization of MIPs was aimed at application in purification and separation, pioneered by companies such as MIP Technologies, POLYINTELL (AFFINIMIPSPE products), and Supelco (previously Sigma Aldrich, now Merck). Acros (SupelMIP) for instance, offers MIPs cartridges for purchase for the solid phase extraction (SPE) of a number of environmental contaminants [238]. Aspira Biosystems sells MIPs, made by epitope imprinting that are able to selectively capture microorganisms [239]. The advantages that MIP offers in this area include savings of time and costs, stability under extreme conditions and no influence of ions on extraction which can be an issue in other porous materials such as zeolites and clays [240], and superior selectivity over standard methods. The reason that early commercialization focused on purification and separation lies in the fact that polymer structure and homogenous binding affinity are not subjected to stringent requirements and bulk microparticles can be directly integrated into columns.

In terms of sensing applications, the main bottlenecks previously identified in previous chapters are the integration of MIPs into electrodes and assays and the large-scale production of homogenous, high affinity MIP particles. Especially the latter can be challenging with standard methods of producing nanoMIPs including electropolymerization or lithographic techniques. Therefore, a few companies have been founded in recent years that focus on synthesizing MIPs on a large-scale for sensing purposes. Semorex synthesizes MIPs for the detection of proteins, with the potential of extending to therapeutics for cancer and treatment of bowel diseases. However, protein imprinted polymers are a challenge in itself since mass-production, if 
conventional approaches are followed, requires a large amount of the template which brings inherent costs.

MIP diagnostics has introduced an alternative approach that might change the field entirely. The company, based in Sharnbrook (UK), was founded in 2015 and focuses on MIP development using the solid-phase imprinting approach to produce high affinity nanoparticles as pioneered by the Piletsky group at the University of Leicester [36]. The advantages of this approach are that the template can be recycled to reduce costs while allowing for (semi) automated production by elution of the polymers instead of the template [37]. Their focus has been contract development work for other companies that have struggled with traditional disadvantages of antibodies, such as batch-to-batch variation or operation under broad temperature and $\mathrm{pH}$ conditions. Their produced nanoMIPs have been applied to remove micropollutants and microorganisms from drinking water, to direct replacement of antibodies in assays, and novel sensor formats. However, recent investment will enable scale-up of operations for development of an in-house portfolio of products, starting with commonly used biomarkers. This can speed up the development of sensing platforms based on these receptors in the future.

Canfarotta et al., reported on the production of nanoMIPs for vancomycin, which were used as antibody-replacements in an ELISA-like format [241]. MIP Diagnostics' nanoMIPs were also integrated into gold screen-printed electrodes for the impedimetric detection of cocaine at trace levels [242]. A simpler and more universal approach to label-free detection of analytes, independent of their electrochemical properties, might be the addition of monomers modified with a redox probe, such as done by Mazzotta et al. who used vinyl ferrocene and ferrocenyl methacrylate to detect vancomycin [243]. The nano-sized homogenous MIPs also meant a huge advantage in thermal MIP-based sensing, allowing to directly couple the nanoparticles to thermocouples for the detection of a wide variety of biomedical targets, ranging from small organic molecules to large proteins at physiologically relevant concentrations [244]. Crapnell et al. further extended this approach by functionalizing multiple thermocouples and integrating them into a single sensor setup for the multiplexed detection of cardiac biomarkers (Figure 11) [59]. The sensor demonstrated to be capable of simultaneously detecting the biomarkers in buffer and serum solutions. Betlem et al. recently introduced an alternative readout approach, using thermistors rather than thermocouples, which simplified analysis by measuring electrical resistance. Furthermore, the use of thermistors offers several advantages such as their low-cost, robustness, and highly sensitive response to small changes in temperature around a fixed base point [245]. 
A

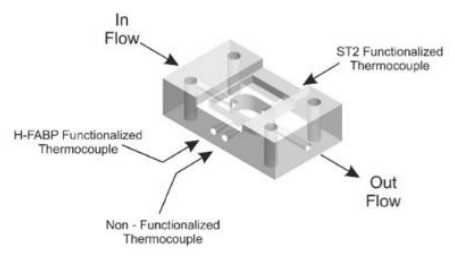

B

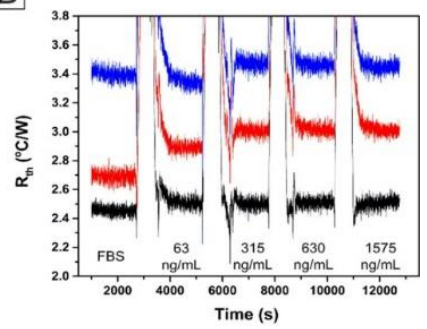

C

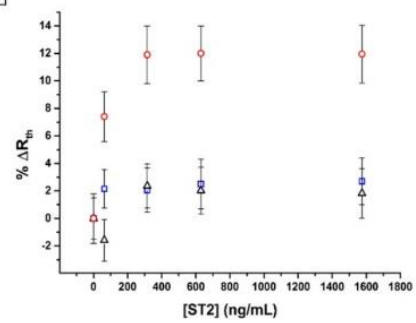

Figure 11. (A) Schematic of the triple thermocouple flow cell used. (B) Raw data HTM plot of the Rth over time for the addition of ST2 in FBS to a triple thermocouple set-up with one thermocouple functionalized with ST2 nanoMIPs (red), one functionalized with $\mathrm{H}$ FABP nanoMIPs (blue), and one unfunctionalized thermocouple (black). (C) Plot of the Rth \% change for the addition of ST2 in FBS to a triple thermocouple set-up with one thermocouple functionalized with ST2 nanoMIPs (red circles), one functionalized with $\mathrm{H}$ FABP nanoMIPs (blue squares), and one unfunctionalized thermocouple (green triangles). Reprinted with permission from [59]. Copyright American Chemical Society 2019.

In the future, companies like MIP Diagnostics hold the key for the commercialization of MIP-based sensing platforms as they focus on overcoming the main bottleneck standing in the way of commercialization. To do so, they will not only have to increase their focus on mass-producing their nanoMIPs but will also have to continue to work with research groups around the world to explore how these MIPs can be integrated into sensors. In doing so, they can profit from the knowhow of companies that are traditionally involved in the construction of biosensor and assay platforms. An exciting advancement recently announced by the company is the collaboration with Stream Bio, an enterprise that is specialized in bioimaging. The goal is to combine nanoMIPs and conjugated magnetic nanoparticles in a lateral flow assay for the development of a fast (10 minutes) diagnostic test for SARS-CoV2. The test could improve the diagnostic capacity of healthcare systems worldwide by offering PoC diagnostics but can also be used to screen sewage 
water for SARS-COV-2. The latter is seen as a potential path of spreading the disease [246]. In the future, one would expect more assay formats that incorporate MIPs and there is potential towards the area of oral therapeutics and drug delivery due to the nanoMIPs inherent biocompatibility [247].

\section{Outlook}

The literature analysis done in this review re-emphasizes the commercial potential of MIPs as alternatives for bioreceptors in sensor platforms and assay formats but also illustrates why commercialization of MIP-based sensors and assays lags somewhat behind in comparison to biosensors and assays based on natural receptors. This can be attributed in part to historical reasons. Biosensors emerged in an era when personalized medicine did not really exist yet while the need was there. Home-based self-testing for blood glucose levels was not possible using traditional, state-of-the-art detection platforms but it has changed the life of diabetics around the globe. Likewise, home pregnancy testing allowed women and couples in general, to assess their pregnancy status without needing to overcome the social and financial threshold of visiting a doctor every time. Biosensors filled a void that traditional laboratory analysis could not fill and acquired market relevance and momentum because of it. This momentum, and resulting financing, in combination with prior knowledge on coupling enzymes and antibodies to surfaces, has formed the basis of the success story of these platforms that have become ubiquitous in modern-day society.

Despite these success stories, traditional biosensors for personalized medicine have still not fulfilled their full potential, as lab-based analysis remains the golden standard due to the high sensitivity and high throughput associated with techniques such as PCR, LC- and GC-MS... However, gradually society is evolving and technological developments that are making their way into society such as smartphones and 3D printing have accelerated the development of e-health and biosensing platforms. MIP-based sensors and assays could also profit from this trend. Many of the examples highlighted in this review, illustrate how MIPs are compatible with smartphone readout, wearable sensors or lateral flow assays based on 3D-printed microfluidic platforms. This research has tackled one of the main bottlenecks traditionally hindering MIP-based sensing from being commercial: integration of MIP particles in electrodes and assay formats suitable for commercial production.

The main challenge remains to create MIPs in large batches that are homogenous in size and shape but also in their affinity towards their target. Recent advances in imprinting technology have led to MIP particles with 
affinities that are comparable to that of natural receptors, which is necessary to convince the market that MIPs have a benefit and create momentum in a manner similar to the first biosensors. Companies such as MIP Diagnostics that come up with ways to not only improve the affinity of the target but also enable the large-scale production of homogenous batches of these particles, hold the key to commercial success. These new methodologies could compete with new advances in reactor synthesis of antibodies and enzyme. In this way, MIPs will no longer have a technological disadvantage over their natural counterparts and their benefits including low-cost price and superior mechanical, chemical and thermal stability will become more important in commercial diagnostics. In addition to the generic nature of the technology, which, in contrast to their natural counterparts, allows for the detection of nearly any target of nearly any size, these characteristics of MIPs still makes molecular imprinting a very interesting, commercially viable technology that can disrupt the diagnostic market for years to come. 


\section{Chapter 2 References}

[1] Newman, J. D.; Turner, A. P. F. Home blood glucose biosensors: A commercial perspective. Biosensors and Bioelectronics. 2005, 20(12), 2435-2453.

[2] Gnoth. C.; Johnson, S. Strips of hope: Accuracy of home pregnancy tests and new developments. Obstetrics Frauenheilkd. 2014, 74(7), 661-669.

[3] Fan, Y.; Zeng, G.; Ma, X. Effects of prepolymerization on surface molecularly imprinted polymer for rapid separation and analysis of sulfonamides in water. J. Colloid \& Interface Sci. 2020, 571, 21-29.

[4] Lowdon, J. W.; Alkirkit, S. M. O.; Mewis, R. E.; Fulton, D.; Banks, C. E.; Sutcliffe, O. B.; Peeters, M. Engineering molecularly imprinted polymers (MIPs) for the selective extraction and quantification of the novel psychoactive substance (NPS) methoxphenidine and its regioisomers. Analyst. 2018, 143, 2081-2089.

[5] Eersels, K.; Grinsven, B.; Vandenryt, T.; Jimenez-Monroy, K. L.; Peeters, M.; Somers, V.; Putmann, C.; Stein, C.; Barth, S.; Bos, G.; Germeraad, G.; Cleij, T. J.; Theolan, R.; Ceuninck, W.; Wagner, P. Selective Identification of Macrophages and Cancer Cells Based on Thermal Transport through Surface-Imprinted Polymers Layers. Phys. Stat. Solidi A. 2015, 212, 1320-1326.

[6] Peeters, M.; Troost, F. J.; Mingels, R. H. G.; Grinsven, B.; Vranken, T.; Ingebrandt, S.; Thoelen, R.; Cleij, T. J.; Wagner, P. Impedimetric Detection of Histamine in Bowel Fluids Using Synthetic Receptors with pH-Optimized Binding Characteristics. Anal Chem. 2013, 85, 1475-83.

[7] Wackers, G.; Putzeyz, T.; Peeters, M.; Cauter, L.; Cornelis, P.; Wubbenhorst, M.; Tack, J.; Troost, F.; Verhaert, N.; Doll, T.; Wagner, P. Towards a catheter-based Impedimetric sensor for the assessment of intestinal histamine levels in IBS patients. Biosensors $\mathcal{E}$ Bioelectronics. 2020, 158, 112152.

[8] Daniels, M.; Dines, M.; Petach, H. H. The denaturation and degradation of stable enzymes at high temperatures. Biochem J. 1996, 317, 1-11.

[9] Abdel-Haleem, F. M.; Madbouly, A.; El Nashar, R. M.; Abdel-Ghani, N. T. Molecularly Imprinted Polymer-based bulk optode for the determination of itopride hydrochloride in physiological fluids. Biosensors \&Bioelectronics. 2016, 85, 740-742.

[10] Chen, S.; Fu, J.; Li, Z.; Zeng, Y.; Li, Y.; Su, X.; Jiang, X.; Yang, H.; Huang, L.; Zou, L.; $\mathrm{He}, \mathrm{L}$; Liu, S.; Ao, X.; Yang, Y. Preparation and application of magnetic molecular imprinted polymers for extraction of cephalexin from pork and milk samples. $J$ Chromatography A. 2019, 1602, 124-134.

[11] Bashir, K.; Guo, P.; Chen, G.; Li, Y.; Ge, Y.; Shu, H.; Fu, Q. Synthesis, characterization, and application of grisepfulvin surface molecularly imprinted polymers as the selective solid phase extraction sorbent in rat sample samples. Arabian J Chem. 2020, 13, 4082-4091. [12] Aghoutane, Y.; Diouf, A.; Osterlund, L.; Bouchikhi, B.; El Bari, N. Development of a molecularly imprinted polymer electrochemical sensor and its application for sensitive detection and determination of malathion in olive fruits and oils. Bioelectrochemisrty. 2020, $132,107404$.

[13] Arak, H.; Torshizi, M. A. K.; Hedayati, M.; Rahimi, S. Comparative evaluation of aflatoxin and mineral binding activity of molecular imprinted polymer designed for dummy template using in vitro and in vivo models. Toxicon. 2019, 166. 66-75.

[14] Cantarella, M.; Carroccio, S. C.; Dattilo, S.; Avolio, R., Castaldo, R.; Puglisi, C.; Privitera, V. Molecularly imprinted polymer for the selective absorption of diclofenac from contaminated water. Chem Eng J. 2019, 367, 180-188. 
[15] Hammam, M. A.; Wagby, H. A.; El Nashar, R. M. Moxifloxacin hydrochloride electrochemical detection base don newly designed molecularly imprinted polymer. Sensors \& Actuators B: Chem. 2018, 275, 127-136.

[16] Wang, D.; Gao, D.; Huang, Y.; Xu, W.; Xia, Z. Preparation of restricted access molecularly imprinted polymers based fiber for selective solid-phase microextraction of hesperetin and its metabolites in vivo. Talanta. 2019, 202, 392-401.

[17] Kumar, N.; Narayanan, N.; Gupta, S. Ultrasonication addicted extraction of chlorpyrifos from honey and brinjal using magnetic molecularly imprinted polymers followed by GLC-ECD analysis. Reactive \& Functional Polymers. 2019, 135, 103-112.

[18] Mudd, S. A hypothetical mechanism of antibody formation. J of Immunology. 1932, 23, 423-427.

[19] Polyakov, M. V.; Stadnik, P.; Paryckij, M.; Malkin, I.; Duchina, F. On the structure of silica. Zhurnal Fizieskoj Kihimii. 1933, 4, 454-456.

[20] Polyakov, M.; Kuleshina, L.; Neimark, I. On the dependence of silica gel absorption properties on the character of its porosity. Zhurnal Fizieskoj Kihimii. 1937, 10, 100-112.

[21] Tang, Y.; Lan, J.; Gao, X.; Liu, X.; Xhang, D.; Wei, L.; Gao, Z.; Li, J. Determination of clenbuterol in pork and potable water samples by molecularly imprinted polymer through the use of covalent imprinting method. Food Chem. 2016, 190, 952-959.

[22] Qi, P.; Wang, J.; Wang, L.; Li, y.; Jin, J.; Su, F.; Tian, Y.; Chen, J. Molecularly imprinted polymers synthesized via semi-covalent imprinting with sacrificial spacer for imprinting phenols. Polymer. 2010, 51, 5417-5423.

[23] Yusof, N. N. M.; Tanioka, E.; Kobayashi, T. Molecularly imprinted polymer particles having coordination hydrogen bonding in covalent-imprinting for efficient recognition towards vanillin. Separation \& Purification Tech. 2014, 122, 341-349.

[24] Golker, K.; Olsson G. D.; Nicholls, I. A. The influence of a methyl substituent on molecularly imprinted polymer morphology and recognition - Acrylic acid versus methacrylic acid. European Polymer J. 2017, 92, 137-149.

[25] Siripairoj, W.; Kaewchada, A.; Jaree, A. Synthesis of molecularly imprinted polymers for the separation of gamma-oryzanol by using methacrylic acid as functional monomer. J of the Taiwan Institute of Chem Eng. 2014, 45, 338-346.

[26] Sobiech, M.; Maciejewska, D.; Lulinski, P. Synthesis and characterization of poly(methacrylic acid-co-trimethylolpropane trimethacrylate) imprinted sorbent for analysis of biogenic amines. Materials Today Comm. 2020, 22, 100739.

[27] Ji, K.; Luo, X.; He, L.; Liao, S.; Hu, L.; Han, J.; Chen, C.; Liu, Y.; Tan, N. Preparation of hollow magnetic molecularly imprinted polymer and its application in silybin recognition and controlled release. J Pharmaceutical \& Biomedical Anal. 2020, 180, 113036.

[28] Dai, C. M.; Zhou, X. F.; Zhang, Y. L.; Liu, S. G.; Zhang, J. Synthesis by precipitation polymerization of molecularly imprinted polymer for the selective extraction of diclofenac from water samples. J of Hazardous Materials. 2011, 198, 175-181.

[29] Shen, X.; Huang, C.; Shinde, S.; Switnickaplak, M.; Cormack, P. A. G.; Sellergren, B. Reflux precipitation polymerization: a new synthetic insight in molecular imprinting at high temperature. RSC Advances. 2016, 6, 81491-81499.

[30] Pardeshi, S.; Singh, S. K. Precipitation polymerization: A versatile tool for preparing molecularly imprinted polymer beads for chromatography applications. RSC Adv. 2016, 6, 23525-23536. 
[31] Sun, Y.; Ren, T.; Deng, Z.; Yang, Y.; Zhong, S. Molecularly imprinted polymers fabricated using Janus particl-stabilized Pickering emulsions and charged monomer polymerization. New J. Chem. 2018, 42, 7355-7363.

[32] Wei, S.; Molinelli, A.; Mizaikoff, B. Molecularly imrpinted micro and nanospheres for the selective recognition of 17 $\beta$-estradiol. Bioesnsors and Bioelectronics. 2006, 21, 1943-1951. [33] Zhoa, G.; Liu, J.; Liu, M.; Han, X.; Peng, Y.; Tian, X.; Liu, J.; Zhang, S. Synthesis of molecularly imprinted polymer via emulsion polymerization for application in solanesol separation. Appl. Sci. 2020, 10(8), 2868.

[34] Panagiotopoulou, M.; Salinas, Y.; Beyazit, S.; Kunath, S.; Duma, L.; Prost, E.; Mayes, A. G.; Resmini, M.; Bui, B. T. S.; Haupt, K. Molecularly imprinted polymer coated quantum dots for multiplexed cell targeting and imaging. Chem. Int. Ed. 2016, 55, 8244-8248.

[35] Wang, S.; Yin, D.; Wang, W.; Shen, X.; Zhu, J.; Chen, H. Y.; Li, Z. Targeting and imaging of cancer cells via monosaccharide-imprinted fluorescent nanoparticles. Sci. Rep. 2016, 6, 22757.

[36] Poma, A.; Guerreiro, A.; Whitcombe, M. J.; Piletska, E. V.; Turner, A. P. F.; Piletsky, S. A. Solid-phase synthesis of molecularly imprinted polymer nanoparticles with a reusable template-"Plastic Antibodies". Adv. Funct. Mater. 2013, 23, 2821-2827.

[37] Canfarotta, F.; Poma, A.; Guerreiro, A.; Piletsky, S. Solid-phase synthesis of molecularly imprinted nanoparticles. Nat. Protoc. 2016, 11, 443-445.

[38] Ambrosini, S.; Beyazit, S.; Haupt, K.; Bui, B. T. S. Solid-phase synthesis of molecularly imprinted nanoparticles for protein recognition. Chem. Commun. 2013, 49, 6746-6748.

[39] Xu, J.; Medina-Rangel, P. X.; Haupt, K.; Bui, B. T. S. Chapter six - guide to the preparation of molecularly imprinted polymer nanoparticles for protein recognition by solid phase synthesis. Methods in Enzymology. 2017, 590, 115-141.

[40] Xu, J.; Ambrosii, S.; Tarnahkar, E.; Rossi, C.; Haupt, K.; Bui. B. T. S. Towards a universal method for preparing molecularly imprinted polymer nanoparticles with antibody-like affinity for proteins. Biomacromolecules. 2016, 17, 345-353.

[41] Xu, J.; Haupt, K.; Bui. B. T. S. Core-shell molecularly imprinted polymer nanoparticles as synthetic antibodies in a sandwich fluoroimmunoassay for trypsin determination in human serum. Appl. Mater. Interfaces. 2017, 9, 24476-24483.

[42] Mosbach, K. Molecular Imprinting. Trends in Biochem Sci. 1994, 19, 9-14.

[43] Piletsky, S. A.; Parhometz, Y. P.; Lavryk, N. V.; Panasyuk, T. L.; El'skaya, A. V. Sensors for low-weight organic molecules based on molecular imprinting technique. Sesnors $\mathcal{E}$ Actuators B; Chem. 1994, 19, 629-631.

[44] Zhao, X.; He, Y.; Wang, Y.; Wang, S.; Wang, J. Hollow molecularly imprinted polymer based quartz crystal microbalance sensor for rapid detection of methimazole in food samples. Food Chem. 2020, 309, 125787.

[45] Dayal, H.; Ng, W, Y.; Lin, X, H.; Li, S. F. Y. Development of a hydrophilic molecularly imprinted polymer for the detection of hydrophilic targets using quartz crystal microbalance. Sensors \& Actuators B: Chem. 2019, 300, 127044.

[46] Ma, X.; He, X.; Li, W.; Zhang, Y. Epitope molecularly imprinted polymer coated quartz crystal microbalance sensor for the determination of human serum albumin. Sensors $\mathcal{E}$ Actuators B: Chem. 2017, 248, 879-886.

[47] Naklua, W.; Suedee, R.; Lieberzeit, P. A. Dopaminergic receptor-ligand binding assay based on molecularly imprinted polymers on quartz crystal microbalance sensors. Biosensors \& Bioelectronics. 2016, 81, 117-124. 
[48] Fang, G., Liu, G., Yang, Y., \& Wang, S. Quartz crystal microbalance sensor based on molecularly imprinted polymer membrane and three-dimensional $\mathrm{Au}$ nanoparticles@mesoporous carbon CMK-3 functional composite for ultrasensitive and specific determination of citrinin. Sensors E Actuators B: Chem. 2016, 230, 272-280.

[49] Feng, F.; Zheng, J.; Qin, P.; Han, T.; Zhao, D. A novel quartz crystal microbalance sensor array based on molecularly imprinted polymers for simultaneous detection of clenbuterol and its metabolites. Talanta. 2017, 167, 94-102.

[50] Eersels, K.; Grinsven, B.; Ethirajan, A.; Timmermans, S.; Jimenez-Monroy, K. L.; Bogie, J. F. J.; Punniyakoti, S.; Vandenryt, T.; Hendriks, J. J. A.; Cleij, T. J.; Daemen, M. J. A. P.; Somers, V.; Ceuninck, W.; Wagner, P. Selective identification of macrophages and cancer cells based on thermal transport through surface-imprinted polymer layers. ACS App Materials \& Interfaces. 2013, 5, 7258-7267.

[51] Peeters, M.; Csipai, P.; Geerets, B.; Weustenraed, A.; Grinsven, B.; Theolen, R.; Gruber, J.; Ceuninck, W.; Cleij, T. J.; Troost, F. J.; Wagner, P. Heat-transfer-based detection of Lnicotine, histamine, and serotonin using molecularly imprinted polymers as biomimetic receptors. Anal \& Bioanal Chem. 2013, 405, 6453-6460.

[52] Zhang, K.; Kwabena, A. S.; Wang, N.; Lu. Y.; Cao, Y.; Luan, Y.; Liu, T.; Peng, H.; Gu, $\mathrm{X}$.; $\mathrm{Xu}, \mathrm{W}$. Electrochemical assays for the detection of TBBPA in plastic products based on rGO/AgNDs nanocomposites and molecularly imprinted polymers. J Electroanalytical Chem. 2020, 862, 114022.

[53] Udornsap, D.; Brisset, H.; Culioli, G.; Dollet, P.; Laatikainen, K.; Siren, H.; Branger, C. Electrochemical molecularly imprinted polymers as material for pollutant detection. Materials Today Communications. 2018, 17, 458-465.

[54] Motla, S.; Bouchikhi, B.; Llobet, E.; Bari, E. Synthesis and characterization of a highly sensitive and selective electrochemical sensor based on molecularly imprinted polymer with gold nanoparticles modified screen-printed electrode for glycerol determination in wastewater. Talanta. 2020, 216, 120953.

[55] Sadriu, I.; Bouden, S.; Nicolle, J.; Podvorica, F.; Bertagna, V.; Berho, C.; Amalric, L.; Vautrin-Ul, C. Molecularly imprinted polymer modified glassy carbon electrodes for the electrochemical analysis of isproturon in water. Talanta. 2020, 207, 120222.

[56] Eersels, K.; Grinsven, B.; Peeters, M.; Cleij, T. J.; Wagner, P. Heat transfer as a new sensing technique for the label-free detection of biomolecules. Label-Free Biosensing. 2017, 19, 383-407.

[57] Eersels, Grinsven, B.; Vandenryt, T.; Jimenez-Monroy, K. L.; Peeters, M.; Somers, V.; Puttmann, C.; Stein, D.; Barth, S.; Bos, G. M. J.; Germeraad, W. T. V.; Dilien, H.; Cleij, T. J.; Theolen, R.; Ceuninck, W.; Wagner, P. Improving the sensitivity of the heat-transfer method (HTM) for cancer cell detection with optimized sensor chips. Physica Status Solidi (a). 2015, 212, 1320-1326.

[58] Eersels, K.; Dilien, H.; Lowdon, J. W.; Redeker, E. S.; Rogosic, R.; Heidt, B.; Peeters, M.; Cornelis, P.; Lux, P.; Reutelingsperger, C. P.; Schurgers, L. J.; Cleij, T. J.; Grinsven, B. A novel biomimetic tool for assessing vitamin $\mathrm{K}$ status based on molecularly imprinted polymers. Nutrients. 2018, 10, 751.

[59] Crapnell, R.; Canfarotta, F.; Czulak, J.; Johnson, R.; Betlem, K.; Mecozzi, F.; Down, M. P.; Eersels, K.; Grinsven, B.; Cleij, T. J.; Law, R.; Banks, C. E.; Peeters, M. Thermal detection of cardiac biomarkers h-fabp and st2 using molecularly imprinted nanoparticle-based multiplex sensor platform. ACS Sensors. 2019, 4, 2838-2845. 
[60] Betlem, K.; Down, M. P.; Foster, C. W.; Akthar, S.; Eersels, K.; Grinsven, B.; Cleij, T. J.; Banks, C. E.; Peeters, M. Development of a flexible MIP-based biosensor application for the thermal detection of neurotransmitters. MRS Adv. 2018, 3, 1569-1574.

[61] Liu, Y.; Liang, Y.; Yang, R.; Li, J.; Qu, L. A highly sensitive and selective electrochemical sensor based on polydopamine functionalized graphene and molecularly imprinted polymer for the 2,4-dichlorophenol recognition and detection. Talanta. 2019, 195, 691-698.

[62] Scheller, F. W.; Zhang, X.; Yarman, A.; Wollenberger, U.; Gyuresanyl, R. E. Molecularly imprinted polymer-based electrochemical sensors for biopolymers. Current Opinions in Electrochem. 2019, 14, 53-59.

[63] Panahi, Y.; Motaharian, A.; Hosseini, M. R. M.; Mehrpour, O. High sensitive and selective nano-molecularly imprinted polymer based electrochemical sensor for midazolam drug detection in pharmaceutical formulation and human urine samples. Sensors \& Actuators B: Chem. 2018, 273, 1579-1586.

[64] Lowdon, J. W.; Eersels, K.; Rogosic, R.; Heidt, B.; Dilien, H.; Redeker, E. S.; Peeters, M.; van Grinsven, B.; Cleij, T. J. Substrate displacement colorimetry for the detection of diarylethylamines. Sensors and Actuators B: Chem. 2019, 282, 137-144.

[65] Ye, T.; Yin, W.; Zhu, N.; Yuun, M.; Cao, H.; Yu, J.; Gou, Z.; Wang, X.; Zhu, H.; Reyihanguli, A.; $\mathrm{Xu}, \mathrm{F}$. Colorimetric detection of pyrethroid metabolite by using surface molecularly imprinted polymer. Sensors \& Actuators B; Chem. 2018, 254, 417-423.

[66] Wang, M.; Fu, Q.; Zhang, K.; Wan, Y.; Wang, L.; Gao, M.; Xia, Z.; Gao, D. A magnetic and carbon dot based molecularly imprinted composite for fluorometric detection of 2,4,6trinitrophenol. Microchim Acta. 2019, 186, 86.

[67] Olczuk, D.; Priefer, R. A history of continuous glucose monitors (CGMs) in selfmonitoring diabetes mellitus. Diabetes \& metabolic synd; Clinical research \& reviews. 2018, 12, 181-187.

[68] Ahmad, O. S.; Badwell, T. S.; Esen, C.; Garcia-Cruz, A.; Piletsky, S. A. Molecularly imprinted polymers in electrochemical and optical sensors. Trends in Biotech. 2019, 37, 294309.

[69] Li, R.; Feng, Y.; Pan, G.; Liu, L. Advances in molecularly imprinting technology for bioanalytical applications. Sesnors. 2019, 19, 177.

[70] Mattiasson, B.; Truta, L. A. A. N. A.; Sales, M. G. F.; Moreira, F. T. C. Imprinting technology in electrochemical biomimetic sensors. Sensors. 2017, 17, 523.

[71] Sergeyyeva, T. A.; Piletsky, S. A.; Brovko, A. A.; Slinchenko, E. A.; Sergeeva, L. M.; El'skaya, A. V. Selective recognition of atrazine by molecularly imprinted polymer membranes. Development of conductometric sensor for herbicides detection. Analytica Chimica Acta. 1999, 392, 105-111.

[72] Suedee, R.; Intakong, W.; Dickert, F. L. Molecularly imprinted polymer-modified electrode for on-line conductometric monitoring of haloacetic acids in chlorinated water. Analytica Chimica Acta. 2006, 569, 66-75.

[73] Yaroshenko, I.; Kirsanov, D.; Marjanovic, M.; Lieberzeit, P. A.; Korostynska, O.; Mason, A.; Frau, I.; Legin, A. Real-time wáter quality monitoring with chemical sensors. Sesnors. 2020, 20(12), 3432.

[74] Warwick, C.; Guerreiro, A.; Gomez-Caballero, A.; Wood, E.; Kitson, J.; Robinson, J.; Soares, A. Conductance based sensing and analysis of soluble phosphates in wastewater. Biosensors \& Bioelectronics. 2014, 52, 173-179. 
[75] Grieshaber, D.; MacKenzie, R.; Voros, J.; Reimhult, R. Electrochemical biosensors Sensor principles and architectures. Sensors. 2008, 8, 1400-1458.

[76] Gui, R.; Jin, H.; Guo, H.; Wang, Z. Recent advances and future prospects in molecularly imprinted polymers-based electrochemical biosensors. Biosensors $\mathcal{E}$ Bioelectronics. 2018, 100, 56-70.

[77] Chen, L.; Wang, X.; Lu, W.; Wu, X.; Li, J. Molecular imprinting; prespective and applications. Chem. Soc. Rev. 2016, 45, 2137-2211.

[78] Piletsky, S. A.; Turner, A. P. F. Electrochemical sensors based on molecularly imprinted polymers. Electroanalysis. 2001, 14, 317-323.

[79] Wackers, G.; Putzeys, T.; Peeters, M.; Cauter, L.; Cornelis, P.; Wubbenhorst, M.; Tack, J.; Troost, F.; Verhaert, N.; Doll, T.; Wagner, P. Towards a catheter-based impredimetric sensor for the assessment of instestinal histamine levels in IBS patients. Biosensors $\mathcal{E}$ Bioelectronics. 2020, 158, 112152.

[80] Cai, D.; Ren, L.; Zhao, H.; Xu, C.; Zhang, L.; Yu, Y.; Wang, H.; Lan, Y.; Roberts, M. F.; Chuang, F. H.; Naughton, M. F.; Ren, Z.; Chiles, T. C. A molwcular-imprint nanosensor for ultrasensitive detection of proteins. Nature Nanotech. 2010, 5, 597-601.

[81] Su, L.; Jia, W.; Hou, C.; Lei, Y. Microbial biosensors: A review. Biosensors $\mathcal{E}$ Bioelectronics. 2011, 26, 1788-1799.

[82] Cheng, J.; Li, Y.; Zhong, J.; Lu, Z.; Wang, G.; Sun, M.; Jiang, Y.; Zou, P.; Wang, X.; Zhoa, Q.; Wang, Y.; Rao, H. Molecularly imprinted electrochemical sensor based on biomass carbon decorated with $\mathrm{MOF}$-derived $\mathrm{Cr}_{2} \mathrm{O}_{3}$ and silver nanoparticles for selective and sensitive detection of nitrofurazone. Chem. Eng. J. 2020, 398, 125664.

[83] Rezaei, B.; Boroujeni, M.; Ensafi, A. A. Development of Suden II sensor based on modified treated pencil graphite electrode with DNA, o-phenylenediamine, and gold nanoparticle bioimprinted polymer. Sensors \& Actuators B; Chem. 2016, 222, 849-856.

[84] Ghanbari, K.; Roushani, M. A nanohybrid probe based on double recognition of an aptamer MIP grafted onto a MWCNTs-Chit nanocomposite for sensing hepatitis $\mathrm{C}$ virus core antigen. Sensors \& Actuators B; Chem. 2018, 258, 1066-1071.

[85] Zhang, Q.; Jiang, D.; Xu, C.; Ge, Y.; Liu, X.; Wie, Q.; Huang, L.; Ren, X.; Wang, C.; Wang, Y. Wearable electrochemical biosensor based on molecularly imprinted Ag nanowires for noninvasive monitoring lactate in human sweat. Sensors \& Actuators B: Chem. 2020, 320, 128325.

[86] Pacheco, J. G.; Silva, M. S. V.; Freitas, M.; Nouws, H. P. A.; Delerue-Matos, C. Molecularly imprinted electrochemical sensor for the point-of-care detection of breast cáncer biomarker (CA 15-3). Sesnors \& Actuators B:Chem. 2018, 256, 905-912.

[87] Karimian, N.; Stortini, A. M.; Moretto, L. M.; Costantino, C.; Bogialli, S.; Ugo, P. Electrochemosensor for trace analysis of perfluorooctancesulfonate in water based on a molecularly imprinted poly(o-phenylenediamine) polymer. ACS Sensors. 2018, 3(7), 12911298.

[88] Lahcen, A. A.; Baleg, A. A.; Baker, P.; Iwuoha, E.; Amine, A. Synthesis and electrochemical characterization of nanostructured magnetic molecularly imprinted polymers for 17- $\beta$-estradiol determination. Sensors \& Actuators B: Chem. 2017, 241, 698-705. [89] Khadem, M.; Faribod, F.; Norouzi, P.; Foroushani, A. R.; Ganjali, M. R.; Shahtaheri, S. J.; Yarahmadi, R. Modification of carbon paste electrode based on molecularly imprint polymer for electrochemical determination of diazinon in biological and environmental samples. Electroanalysis. 2017, 29, 708-715. 
[90] Apetrei, C.; Ghasemi-Varnamkhasti, M. Chapter 11 - Biosensors in food PDO authentication. Comprehensive analytical Chem. 2013, 60, 279-297.

[91] Kriz, D.; Mosbach, K. Competitive amperometric morphine sensor based on an agarose immobilised molecularly imprinted polymer. Analaytica Chimica Acta. 1995, 300, 71-75.

[92] Ramanaviciene, A.; Ramanavicius A. Molecularly imprinted polypyrrole-based syntehtic receptor for direct detection of bovine leukemia virus glycoproteins. Biosensors \& Bioelectronics. 2004, 20, 1076-1082.

[93] Xue, C.; Han, Q.; Wang, Y.; Wu, J.; Wen, T.; Wang, R.; Hong, J.; Zhou, X.; Jiang, H. Amperometric detection of dopamine in human serum by electrochemical sensor based on gold nanoparticles doped molecularly imprinted polymers. Biosensors \& Bioelectronics. 2013, 49, 199-203.

[94] Yang, Q.; Wu, X.; Peng, H.; Fu, L.; Song, X.; Li, J.; Xiong, H.; Chen, L. Simultaneous phase-inversion and imprinting based sensor for highly sensitive and selective detection of bisphenol A. Talanta. 2018, 176, 595-603.

[95] Yang, Q.; Li, N.; Chen, S.; Wang, H.; Yang, H. Amperometric sarcosine biosensor based on hollow magnetic Pt-Fe3 $\mathrm{O}_{4} @ \mathrm{C}$ nanospheres. Analytic Chimica Acta. 2019, 1078, 161167.

[96] Beluomini, M. A.; Silva, J. L.; Sedenho, G. C.; Stradiotto, N. R. D-mannitol sensor base don molecularly imprinted polymer on electrode modified with reduced Graphene oxide decorated with gold nanoparticles. Talanta. 2017, 165, 231-239.

[97] Almeida, J. M. S.; Toloza, C. A. T.; Machado, B. S.; Silva, A. R.; Aucelio, R. Q. Determination of gentamicin sulfate by batch-injection amperometry after solid-phase extraction using kanamycin-template imprinted polymer. Microchem J. 2019, 145, 187-195. [98] Fogel, R., Limson, J., \& Seshia, A. A. Acoustic biosensors. Essays Biochem. 2016, 60, 101110 .

[99] Dabrowski, M.; Cieplak, M.; Sharma, P. S.; Borowicz, P.; Noworyta, K.; Lisowski, W.; D'Souza, F.; Kuhn, A.; Kunter, W. Hierarchiral templating in deposition of semicovalently imprinted inverse opal polythiophene film for femtomolar determination of human serum albumin. Biosensors \& Bioelectronics. 2017, 94, 155-161.

[100] Dabrowski, M.; Ziminska, A.; Kalecki, J.; Cieplak, M.; Lisowski, W.; Maksym, R.; Shao, S.; D'Souza, F.; Kuhn, A.; Sharma, P. S. Facile fabrication of surface-imprinted macroporous films for chemosensing of human chorionic gonadotropin hormone. ACS appl. Mater. Interfaces. 2019, 11, 9265-9276.

[101] Dabrowski, M.; Sharma, P. S.; Iskierko, Z.; Noworyta, K.; Cieplak, M.; Lisowski, W.; Oborska, S.; Kuhn, A.; Kunther, W. Early diagnosis of fungal infections using piezomicrogravimetric and electric chemosensors based on polymers molecularly imprinted with d-arabitol. Biosensors \& Bioselectronics. 2016, 79, 627-635.

[102] Tamboli, V. K.; Bhalla, N.; Jolly, P.; Bowen, C. R.; Taylor, J. T.; Bowen, J. L.; Allender, C. J.; Estrela, P. Hybrid synthetic receptors on MOSFET devices for detection of prostate specific antigen in human plasma. Anal. Chem. 2016, 88, 11486-11490.

[103] Kajisa, T.; Sakata, T. Molecularly imprinted artificial biointerface for an enzyme-free glucose transistor. ACS Appl. Mater. Interfaces. 2018, 41, 34983-34990.

[104] Iskierko, Z.; Sharma, P. S.; Noworyta, K. R.; Borowicz, P.; Cieplak, M.; Kunter, W.; Bossi, A. M. Selective PQQPFPQQ gluten epitope chemical sensor with a molecularly imprinted polymer recognition unit and an extended-gate field-effect transistor transduction unit. Anal. Chem. 2019, 91, 4537-4543. 
[105] Ballantine D.S.; White R.M.; Martin S.J.; Ricco A.J.; Zellers E.T.; Frye G.C.; et al. Acoustic Wave Sensors: Theory, Design and Physico-Chemical Applications. San Diego: Academic Press; 1997.

[106] Ayankojo, A. G.; Tretjakov, A.; Reut, J.; Boroznjak, R.; Opik, A.; Rappich, J.; Furchner, A.; Hinrichs, K.; Syritski, V. Molecularly imprinted polymer integrated with a surface acoustic wave technique for detection of sulfamethizole. Anal. Chem. 2016, 88, 1476-1484. [107] Maouche, N.; Ktari, N.; Bakas, I.; Fourati, N.; Zerrouki, C.; Seydou, M.; Maurel, F.; Chehimi, M. A surface acoustic wave sensor functionalized with a polypyrrole molecularly imprinted polymer for selective dopamine detection. J. Mol. Recognit. 2015, 28, 667-678.

[108] Wen, W.; Shitang, H.; Shunzhou, L.; Minghua, L.; Yong, P. Enhanced sensitivity of SAW gas sensor coated molecularly imprinted polymer incorporating high frequency stability oscillator. Sensors \& Actuators B; Chem. 2007, 125, 422-427.

[109] Tretjakov, A.; Syritski, V.; Reut, J.; Boroznjak, R.; Opik, A. Molecularly imprinted polymer film interfaced with surface acoustic wave technology as a sensing platform for label-free protein detection. Analytica Chimica Acta. 2016, 902, 182-188.

[110] Mazouz, Z.; Rahali, S.; Fourati, N.; Zerrouki, C.; Aloui, N.; Seydou, M.; Yaakoubi, N.; Chehimi, M. M.; Othmane, A.; Kalfat, R. Highly selective polypyrrole MIP-based gravimetric and electrochemical sensors for picomolar detection of glyphosate. Sensors. 2017, 17, 2586.

[111] Kidakova, A.; Boroznjak, R.; Reut, J.; Opik, A.; Saarma, M.; Syritski, V. Molecularly imprinted polymer-based SAW sensor for label-free detection of cerebral dopamine neurotrophic factor protein. Sesnors \& Actuators b. 2020, 308, 127708.

[112] Matsuguchi, M.; Uno, T. Molecular imprinting strategy for solvent molecules and its applications for QCM-based VOC sensing. Sesnors \& Actuators B. 2006, 113, 94-99.

[113] Lu, C., Zhang, Y.; Tang, S.; Fang, Z.; Yang, H.; Chen, X.; Chen, G. Sensing HIV related protein using epitope imprinted hydrophilic polymer coated quartz crystal microbalance. Biosensors \& Bioelectronics. 2012, 31, 439-444.

[114] Sener, G.; Ozgur, E.; Yilmaz, E.; Uzun, L.; Say, R.; Denizli, A. Quartz crystal microbalance based nanosensor for lysozyme detection with lysozyme imprinted nanoparticles. Biosensors \& Bioelectronics. 2010, 26, 815-821.

[115] Sauerbrey, G. Use of quartz crystals for weighing then layers and for micro weighing. Z. Physik. 1959, 155, 206-222.

[116] Ersoz, A.; Diltemiz, S. E.; Ozcan, A. A.; Denizli, A.; Say, R. 8-OHdG sensing with MIP based solid phase extraction and QCM technique. Sensors \& Actuators B. 2009, 137, 7-11.

[117] Qian, K.; Deng, Q.; Fang, G.; Wang, J.; Pan, M.; Wang, S.; Pu, Y. Metal-organic frameworks supported surface-imprinted nanoparticles for the sensitive detection of metolcarb. Biosensors \& Bioelectronics. 2016, 79, 359-363.

[118] Jha, S. K., Liu, C.; Hayashi, K. Molecular imprinted polyacrylic acids based QCM sensor array for recognition of organic acids in body odor. Sensors \& Actuators B. 2014, 204, 74-87.

[119] Lieberzeit, P. A.; Rehman, A.; Najafi, B.; Dickert, F. L. Real-life application of a QCMbased e-nose: quantitative characterization of different plant-degradation processes. Bioanal Chem. 2008, 291, 2897-2903.

[120] Tai, D.; Lin, C.; Wu, T.; Chen, L. Recognition of dengue virus protein using epitopemediated molecularly imprinted film. Anal. Chem. 2005, 77, 5140-5143. 
[121] Lin, T.; Hu, C.; Chou, T. Determination of albumin concentration by MIP-QCM sensor. Biosensors \& Bioelectronics. 2004, 20, 75-81.

[122] Tai, D.; Jhang, M.; Chen, G.; Wang, S.; Lu, K.; Lee, Y.; Liu, H. Epitope-cavities generated by molecularly imprinted films measure the coincident response to anthrax protective antigen and its segments. Anal. Chem. 2010, 82, 2290-2293.

[123] Dickert, F. L.; Hayden, O.; Bindeus, R.; Mann, K.; Blaas, D.; Waigmann, E. Bioimprinted QCM sensors for virus detection-screening of plant sap. Anal Bioanal Chem. 2004, 378, 1929-1934.

[124] Lieberzeit, P. A.; Chunta, S.; Navakul, K.; Sangma, C.; Jungmann, C. Molecularly imprinted polymers for diagnostics; Sensing high density lipoprotein and dengue virus. Procedia Eng. 2016, 168, 101-104.

[125] Tai, D.; Lin, C.; Wu, T.; Huang, J.; Shu, P. Artificial receptors in serologic tests for the early diganosis of dengue virus infection. Clinical Chemistry. 2006, 52, 1486-1491.

[126] Pollar, A.; Spieker, E.; Lieberzeit, P. A.; Preininger, C. Surface imprints; Advantageous application of ready2use materials for bacterial quartz-crystal microbalance sensors. ACS. Appl. Mater. Interfaces. 2017, 9, 1129-1135.

[127] Latif, U.; Qian, J.; Can, S.; Dickert, F. L. Biomimetic receptors for bioanalyte detection by quartz crystal microbalances - from mlecules to cells. Sensors. 2014, 14, 23419-23438.

[128] Ladenhauf, E. M.; Pum, D.; Wastl, D. S.; Toca-Herrera, J. L.; Phan, N. V. H.; Lieberzeit, P. A.; Sleytr, U. B. S-layer based biomolecular imprinting. RSC. Adv. 2015, 5, 83558-83564.

[129] Jenik, M.; Seifner, A.; Krassnig, S.; Seidler, K.; Lieberzeit, P. A.; Dickert, F. L.; Jungbauer, C. Sensors for bioanalytes by imprinting-polymers mimicking both biological receptors and the corresponding bioparticles. Biosensors \& Bioelectronics. 2009, 25, 9-14.

[130] Hayden, O.; Mann, K.; Mag, S.; Dickert, F. L. Biomimetic ABO blood-group typing. Angew. Chem. Int. Ed. 2006, 45, 2626-2629.

[131] Battal, D.; Akgonullu, S.; Yalcin, M. S.; Yavuz, H.; Denizli, A. Molecularly imprinted polymer based quartz crystal microbalance sensor system for sensitive and label-free detection of synthetic cannabinoids in urine. Biosensors \& Bioelectronics. 2018, 111, 10-17.

[132] Tai S.; Fantegrossi W.E. Pharmacological and Toxicological Effects of Synthetic Cannabinoids and Their Metabolites. 2016 In: Baumann M., Glennon R., Wiley J. (eds) Neuropharmacology of New Psychoactive Substances (NPS). Current Topics in Behavioral Neurosciences, vol 32. Springer, Cham.

[133] Chunta, S.; Suedee, R.; Lieberzeit, P. A. High-density lipoprotein sensor based on molecularly imprinted polymer. Anal Bioanal Chem. 2018, 410, 875-883.

[134] Chunta, S.; Suedee, R.; Lieberzeit, P. A. High-density lipoprotein sensor based on molecularly imprinted polymer. Anal Chem. 2016, 88, 1419-1425.

[135] Cheubong, C.; Yoshida, A.; Mizukawa, Y.; Hayakawa, N.; Takai, M.; Morishita, T.; Kitayama, Y.; Sunayama, H.; Takeuchi, T. Molecularly imrpinted nanogels capable of porcine serum albumin detection in raw meat extract for halal food control. Anal. Chem. 2020, 92, 6401-6407.

[136] Zhao, X.; He, Y.; Wang, Y.; Wang, S.; Wang, J. Hollow molecularly imprinted polymer based quartz crystal microbalance sensor for rapid detection of methimazole in food samples. Food Chem. 2020, 309, 125787.

[137] Chen, W.; Wang, Z.; Gu, S.; Wang, J. Detection of hexanal in humid circumstances using hydrophobic molecularly imprinted polymers composite. Sensors \& Actuators B. 2019, 291, 141-147. 
[138] Yun, Y.; Pan, M.; Fang, G.; Gu, Y.; Wen, W.; Xue, R.; Wang, S. An electrodeposited molecularly imprinted quartz crystal microbalance sensor sensitized with AuNPs and rGO material for ighly selective and sensitive detection of amantadine. RSC. Adv. 2018, 8, 6600-6607.

[139] Ayankojo, A. G.; Reut, J.; Boroznjak, R.; Opik, A.; Syritski, V. Molecularly imprinted poly(meta-phenylenediamine) based QCM sensor for detecting amoxicillin. Sensors \& Actuators B. 2018, 258, 766-774.

[140] Wang, Z.; Chen, W.; Gu, S.; Wang, J.; Wang, Y. Discrimination of wood borers infested platycladus orientalis trunks using quartz crystal microbalance gas sensor array. Sensors \& Actuators B. 2020, 309, 127767.

[141] Yang, J. C.; Lee, J.; Hong, S. W.; Park, J. Molecularly imprinted quartz crystal microbalance sensors with lithographically patterned frisbee-like pillar arrays for sensitive and selective detection of iprodione. Sensors \& Actuators B. 2020, 320, 128366.

[142] Guha, A.; Ahmad, O. S.; Guerreiro, A.; Karim, K.; Sandstrom, N.; Ostanin, V. P.; Wijngaart, W.; Piletsky, S. A.; Ghosh, S. K. Direct detection of small molecules using a nano-molecular imprinted polymer receptor and a quartz crystal resonator driven at a fixed frequency and amplitude. Biosensors \& Bioelectronics. 2020, 158, 112176.

[143] Klangprapan, S.; Choke-arpornchai, B.; Lieberzeit, P. A.; Choowongkomon, K. Sensing the classical swine fever virus with molecularly imprinted polymer on quartz crystal microbalance. Heliyon. 2020, 6, e04137.

[144] Bhand, S. G.; Yilmaz, E.; Danielsson, B. Coupled biosensor, biomimetic and chemometrics strategies for analysis of the metals in complex environmental matrices. J. Phys. IV France. 2003, 107, 169-172.

[145] Lettau, K.; Warsinke, A.; Katterie, M.; Danielsson, B.; Scheller, F. W. A bifunctional molecularly imprinted polymer (MIP); Analysis of binding and catalysis by a thermistor. Angew Chem Int Ed. 2006, 45.

[146] Athikomrattanakul, U.; Gajovic-Eichelmann, N.; Scheller, F. Thermometric sensing of nitrofurantoin by noncovalently imprinted polymers containing two complementary functional monomers. Anal. Chem. 2011, 83, 7704-7711.

[147] Rajkumar, R.; Katterle, M.; Warsinke, A.; Mohwald, H.; Scheller, F. W. Thermometric MIP sensor for fructosyl valine. Biosensors \& Bioelectronics. 2008, 23, 1195-1199.

[148] Lattau, K.; Katterle, M.; Warsinke, A.; Scheller, F. W. Sequential conversion by catalytically active MIP and immobilized tyrosinase in a thermistor. Biosensors $\mathcal{E}$ Bioelectronics. 2008, 23, 1216-1219.

[149] Grinsven, B.; Bon, N. V.; Strauven, H.; Grieten, L.; Murib, M.; Jimenez Monroy, K. L.; Janssens, S. D.; Haenen, K.; Schoning, M. J.; Vermeeren, V.; Ameloot, M.; Michiels, L.; Theolen, R.; Ceuninck, W.; Wagner, P. Heat-transfer resistance at solid-liquid interfaces; A tool for the detection of single-nucleotide polymorphisms in DNA. ACS Nano. 2012, 6, 2712-2721.

[150] Grinsven, B.; Eersels, K.; Peeters, M.; Losada-perez, P.; Vandenryt, T.; Cleij, T. J.; Wagner, P. The heat-transfer method; A versatile low-cost label-free, fast, and userfriendly readout platform for biosensor applications. ACS Appl. Mater. Interfaces. 2014, 16, 13309-13318.

[151] Peeters, M.; Kobben, S.; Jimenez-monroy, K.; Modesto, L.; Kraus, M.; Vandenryt, T.; Gaulke, A.; Grinsven, B.; Ingebrandt, S.; Junkers, T.; Wagner, P. Thermal detection of 
histamine with a graphene oxide base molecularly imprinted polymer platform prepared by reversible addition-fragmentation chain transfer polymerization. Sensors \& Actuators B. 2014, 203, 527-535.

[152] Vandenryt, T.; Grinsven, B.; Eersels, K.; Cornelis, P.; Kholwadia, S.; Cleij, T. J.; Thoelen, R.; Ceuninck, W.; Peeters, M.; Wagner, P. Single-shot detection of neurotransmitters in whole-blood samples by means of the heat-transfer method in combination with synthetic receptors. Sensors. 2017, 17, 2701.

[153] Eersels, K.; Dilien, H.; Lowdon, J. W.; Redeker, E. S.; Rogosic, R.; Heidt, B.; Peeters, M.; Cornelis, P.; Lux, P.; Reutelingsperger, C. P.; Schurgers, L. J.; Cleij, T. J.; Grinsven, B. A novel biomimetic tool for assessing vitamin $\mathrm{K}$ status based on molecularly imprinted polymers. 2018, 10(6), 751.

[154] Peeters, M.; Grinsven, B.; Cleij, T. J.; Jimenez-monroy, K. L.; Cornelis, P.; Perez-Ruiz, E.; Wackers, G.; Theolen, R.; Ceuninck, W.; Lammertyn, J.; Wagner, P. Lbel-free protein detection based on the heat-transfer method-A case study with the peanut allergen ara $\mathrm{h}$ 1 and amptamer-based synthetic receptors. ACS Appl. Mater. Interfaces. 2015, 19, 1031610323.

[155] Eersels, K.; Grinsven, B.; Ethirajan, A.; Timmermans, S.; Jimenez-monroy, K. L.; Bogie, J. F. J.; Punniyakoti, S.; Vandenryt, T.; Hendriks, J. J. A.; Cleij, T. J.; Daemen, M. J. A. P.; Somers, V.; Ceuninck, W.; Wagner, P. Selective identification of macrophages and cancer cells based on thermal transport through surface-imprinted polymer layers. ACS Appl. Mater. Interfaces. 2013, 15, 7258-7267.

[156] Eersels, K.; Grinsven, B.; Khorshid, M.; Somers, V.; Puttmann, C.; Stein, C.; Barth, S.; Dilien, H.; Bos, G. M. J.; Germeraad, W. T. V.; Cleij, T. J.; Thoelen, R.; Ceuninck, W.; Wagner, P. Heat-transfer-method-based cell culture quality assay through cell detection by surface imprinted polymers. Langmuir. 2015, 31, 2043-2050.

[157] Bers, K.; Eersels, K.; Grinsven, B.; Daemen, M.; Bogie, J. F. J.; Hendriks, J. J. A.; Bouwmans, E. E.; Puttmann, C.; Stein, C.; Barth, S.; Bos, G. M. J.; Germeraad, W. T. V.; Ceuninck, W.; Wagner, P. Heat-transfer resistance measurement method (HTM)-based cell detection at trace levels using a progressive enrichment approach with highly selective cell-binding surface imprints. Langmuir. 2014, 30, 3631-3639.

[158] Grinsven, B.; Eersels, K.; Akkermans, O.; Ellermann, S.; Kordek, A.; Peeters, M.; Deschaume, O.; Bartic, C.; Dilien, H.; Redeker, E. S.; Wagner, P.; Cleij, T. J. Label-free detection of Escherichia coli based on thermal transport through surface imprinted polymers. ACS Sens. 2016, 1(9), 1140-1147.

[159] Redeker, E. S.; Eersels, K.; Akkermans, O.; Royakkers, J.; Dyson, S.; Nurekeyeva, K.; Ferrando, B.; Cornelis, P.; Peeters, M.; Wagner, P.; Dilien, H.; Grinsven, B.; Cleij, T. J. Biomimetic bacterial identification platform based on thermal wave transport analysis (TWTA) through surgace imprinted polymers. ACS infect Dis. 2017, 3(5), 388-397.

[160] Yongabi, D.; Khorshid, M.; Losada-perez, P.; Eersels, K.; Deschaume, O.; D’Haen, J.; Bartic, C.; Hooyberghs, J.; Thoelen, R.; Wubbenhorst, M.; Wagner, P. Cell detection by surface imprinted polymers SIPs: A study to unravel the recognition mechanism. Sensors E Actuators B. 2018, 255, 907-917.

[161] Redeker, R. S.; Eersels, K.; Akkermans, O.; Royakkers, J.; Dyson, S.; Nurekeyeva, K.; Ferrando, B.; Cornelis, P.; Peeters, M.; Wagner, P.; Dilien, H.; Grinsven, B.; Cleij, T. J. Biomimetic bacterial identification platform based on thermal wave transport analysis (TWTA) through surface-imprinted polymers. ACS Infect Dis. 2017, 3(5), 388-397. 
[162] Dilien, H.; Peeters, M.; Royakkers, J.; Harings, J.; Cornelis, P.; Wagner, P.; Redeker, E. S.; Banks, C. E.; Eersels, K.; Grinsven, B.; Cleij, T. J. Label-free detection of small organic molecules by molecularly imprinted polymer functionalized thermocouples; towards in vivo applications. ACS Sens. 2017, 2(4), 583-589.

[163] Lowdon, J. W.; Eersels, K.; Rogosic, R.; Boonen, T.; Heidt, B.; Dilien, H.; Grinsven, B.; Cleij, T. J. Surface grafted molecularly imprinted polymeric receptor layers for thermal detection of the new psychoactive substance 2-methoxphenidine. Sensors \& Actuators A. 2019, 295, 586-595.

[164] Cornelis, P.; Givanoudi, S.; Yongabi, D.; Iken, H.; Duwe, S.; Deschaume, O.; Robbens, J.; Dedecker, P.; Bartic, C.; Wubbenhorst, M.; Schoning, M. J.; Heyndrickx, M.; Wagner, P. Sensitive and specific detection of E Coli using biomimetic receptors in combination with a modified heat-transfer method. Biosensors \& Bioelectronics. 2019, 136, 97-105.

[165] Henry, O. Y. F.; Cullen, D. C.; Piletsky, S. A. Optical interrogation of molecularly imprinted polymers and development of MIP sensors; a review. Anal Bioanal Chem. 2005, 382, 947-956.

[166] Wackerlig, J.; Lieberzeit, P. A. Molecularly imprinted polymer nanoparticles in chemical sensing - synthesis, characterization and application. Sensors \& Actuators B. 2015, 207, 144-157.

[167] Ahmad, O. S.; Bedwell, T. S.; Esen, C.; Garcia-Cruz, A.; Piletsky, S. A. Molecularly imprinted polymers in electrochemical and optical sensors. Trends in Biotech. 2019, 37, 294309.

[168] Altintas, Z.; Gittens, M.; Guerreiro, A.; Thompson, K.; Walker, J.; Piletsky, S.; Tothill, I. E. Detection of waterborne viruses using high affinity molecularly imprinted polymers. Anal. Chem. 2015, 87, 6801-6807.

[169] Yilmaz, E.; Majidi, D.; Ozgur, E.; Denizli, A. Whole cell imprinting based Escherichia coli sensors; A study for SPR and QCM. Sensors \& Actuators B. 2015, 209, 714-721.

[170] Lautner, G.; Kaev, J.; Reut, J.; Opik, A.; Rappich, J.; Syritski, V.; Gyurcsanyi, R. E. Selective artificial receptors based on micropatterned surface-imprinted polymers for label-free detection of proteins by SPR imaging. Adv. Funct. Mater. 2011, 21, 591-597.

[171] Li, X.; Husson, S. M. Absorption of dansylated amino acids on molecularly imprinted surfaces: A surface plasmon resonance study. Biosensors \& Bioelectronics. 2006, 22, 336-348. [172] Matsunaga, T.; Hishiya, T.; Takeuchi, T. Surface plasmon resonance sensor for lysozyme based on molecularly imprinted then films. Analytica Chimica Acta. 2007, 591, 6367.

[173] Riskin, M.; Ben-Amram, Y.; Tel-Vered, R.; Chegel, V.; Almog, J.; Willner, I. Molecularly imprinted $\mathrm{Au}$ nanoparticles composites on $\mathrm{Au}$ surfaces for the surface plasmon resonance detection of pentaerythritol tetranitrate, nitroglycerin, and ethylene glycol dinitrate. Anal. Chem. 2011, 83, 3082-3088.

[174] Riskin, M.; Tel-Vered, R.; Willner, I. Imprinted Au-nanoparticle composites for the ultrasensitive surface plasmon resonance detection of hexahydro-1,3,5-trinitro-1,3,5triazine (RDX). Adv. Mater. 2010, 22, 1387-1391.

[175] Cennamo, N.; D' Agostino, G.; Galatus, R.; Bibbo, L.; Pesavento, M.; Zeni, L. Sensors based on Surface plasmon resonance in a plastic optical fiber for the detection of trinitrotoluene. Sensors \& Actuators B. 2013, 188, 221-226.

[176] Kara, M.; Uzun, L.; Kolayli, S.; Denizli, A. Combining molecular imprinted nanoparticles with surface plasmon resonance nanosensor for chloramphenicol detection in honey. Appl Poly Sci. 2013, 129(4), 2273-2279. 
[177] Altintas, Z. Surface plasmon resonance based sensor for the detection of glycopeptide antibiotics in milk using rationally designed nanoMIPs. Scientific reports. 2018, 8, 11222 .

[178] Luo, Q.; Yu, N.; Shi, C.; Wang, X.; Wu, J. Surface plasmon resonance sensor for antibiotics detection based on photo-initiated polymerization molecularly imprinted array. Talanta. 2016, 161, 797-803.

[179] Shrivastav, A. M.; Mishra, S. K.; Gupta, B. D. Fiber optic SPR sensor for the detection of melamine using molcular imprinting. Sensors \& Actuators B. 2015, 212, 406-410.

[180] Taguchi, Y.; Takano, E.; Takeuchi, T. SPR sensing of bisphenol A using molecularly imprinted nanoparticles immobilized on slab optical waveguide with consecutive parallel $\mathrm{Au}$ and Ag deposition bands coexistent with bisphenol A-immobilized Au nanoparticles. Langmuir. 2012, 28, 7083-7088.

[181] Pernites, R. B.; Ponnapati, R. R.; Advincula, R. C. Surface plasmon resonance (SPR) detection of theophylline via electropolymerized molecularly imprinted polythiophenes. Macromolecules. 2010, 43(23), 9724-9735.

[182] Shrivastav, A. M.; Usha, S. P.; Gupta, B. D. Fiber optic profenofos sensor based on Surface plasmon resonance technique and molecular imprinting. Biosensors $\mathcal{E}$ Bioelectronics. 2016, 79, 150-157.

[183] Lotierzo, M.; Henry, O. Y. F.; Piletsky, S.; Tothill, I.; Cullen, D.; Kania, M.; Hock, B.; Turner, A. P. F. Surface plasmon resonance sensor for domoic acid based on grafted imprinted polymer. Biosensors \& Bioelectronics. 2004, 20, 145-152.

[184] Bergdahl, G. E.; Andersson, T.; Allhorn, M.; Yngman, S.; Timm, R.; Lood, R. In vivo detection and absolute quantification of a secreted bacterial factor from skin using molecularly imprinted polymers in a surface plasmon resonance biosensor for improved diagnostic abilities. ACS Sens. 2019, 4(3), 717-725.

[185] Ozkan, A.; Atar, N.; Yola, M. L. Enhanced Surface plasmon resonance (SPR) signls base don immobilization of core-shell nanoparticles incorporated boron nitride nanosheets; Development of molecularly imprinted SPR nanosensor for anticancer drug, etoposide. Biosensor \& Bioelectronics. 2019, 130, 293-298.

[186] Baldoneschi, V.; Palladino, P.; Banchini, M.; Minunni, M.; Scarano, S. Norepinephrine as new functional monomer for molecular imprinting: An applicative study for the optical sensing of cardiac biomarkers. Biosensors \& Bioelectronics. 2020, 157, 112161.

[187] Xue, J.; Li, D.; Qu, L.; Long, Y. Surface-imprinted core-shell Au nanoparticles for selective detection of bisphenol A based on surface-enhanced raman scattering. Analytica Chimica Acta. 2013, 777, 57-62.

[188] Chang, L.; Ding, Y.; Li, X. Surface molecular imprinting onto silver microspheres for surface enhanced raman scattering. Biosensors \& Bioelectronics. 2013, 50, 106-110.

[189] Fang, G.; Fan, C.; Liu, H.; Pan, M.; Zhu, H.; Wang, S. A novel molecularly imprinted polymer CdSe/ZnS quantum dots for highly selective optosensing of mycotoxin zearalenone in cereal samples. RSC Adv. 2014, 4, 2764-2771.

[190] Feng, S.; Hu, Y.; Ma, L.; Lu, X. Development of molecularly imprinted polymerssurface-enhanced raman spectroscopy/colorimetric dual sensor for determination of chlorpyrifos in apple juice. Sensors \& Actuators B. 2017, 241, 750-757.

[191] Feng, J.; Hu, Y.; Grant, E.; Lu, X. Determination of thiabendazole in orange juice using MISPE-SERS chemosensor. Food Chem. 2018, 239, 816-822. 
[192] Li, H.; Wang, X.; Wang, Z.; Jiang, J.; Qiao, Y.; Wie, M.; Yan, Y.; Li, C. A highperformance SERS-imprinted sensor doped with silver particles of different surface morphologies for selective detection of pyrethriods in rivers. New. J. Chem. 2017, 41, 1434214350.

[193] Castro-Grijalba, A.; Montes-Garcia, V.; Cordero-Ferradas, M. J.; Coronado, E.; PerezJuste, J.; Pastoriza-Santos, I. SERS-based molecularly imprinted plasmonic sensor for highly sensitive PAH detection. ACS Sens. 2020, 5(3), 693-702.

[194] Gnoth C, Johnson S. Strips of Hope: Accuracy of Home Pregnancy Tests and New Developments. Geburtshilfe Frauenheilkd. 2014, 74(7), 661-669.

[195] Yetisen, A. K.; Akram, M. S.; Lowe, C. R. Paper-based microfluidic point-of-care diagnostic devices. Lab Chip. 2013, 13, 2210.

[196] Vlatakis, G.; Andersson, L. I.; Muller, R.; Mosbach, K. Drug assay using antibody mimics made by molecular imprinting. Nature. 1993, 361, 645-647.

[197] Andersson, L. I.; Muller, R.; Vlatakis, G.; Mosbach, K. Mimics oft he binding sites of opioid receptors obtaining by molecular imprinting of enkephalin and morphine. PNAS. 1995, 92(11), 4788-4792.

[198] Andersson, L. I. Application of Molecular Imprinting to the Development of Aqueous Buffer and Organic Solvent based Radioligand Binding Assays for (S)Propranolol. Anal. Chem. 1996, 68, 111-117.

[199] Haupt, K.; Dzgoev, A.; Mosbach, K. Assay system for the herbicide 2,4dichlorophenoxyacetic acid using a molecularly imprinted polymer as an artificial recognition element. Anal. Chem. 1998, 70(3), 628-631.

[200] Piletsky, S. A.; Piletskaya, E. V.; El'skaya, A. V.; Levi, R.; Yano, K.; Karube, I. Optical detection system for triazine based on molecular-imrpinted polymers. Anal. Chem. 1997, 30, 445-455.

[201] Turkewitsch, P.; Wandelt, B.; Darling, G.; Powell, W. S. Fluorescent functional recognition sites through molecular imprinting: A polymer-based fluorescent chemosensor for aqueous cAMP. Anal. Chem. 1998, 70, 2025-2030.

[202] Surugiu, I.; Ye, L.; Yilmax, E.; Dzgoev, A.; Danielsson, B.; Mosbach, K.; Haupt, K. An ezyme-linked molecularly imprinted sorbent assay. Analyst. 2000, 125, 13-16.

[203] Stephenson, C. J.; Shimizu, K. D. Colorimetric and Fluorometric molecularly imprinted polymer sensors and binding assays. Polym Int. 2007, 56, 482-488.

[204] Zhao, Y.; Ma, Y.; Li, H.; Wang, L. Composite QDs@MIP nanospheres for specific recognition and direct fluorescent quantification of pesticides in aqueous medium. Anal. Chem. 2012, 84, 386-395.

[205] Lin, C.I.; Joseph, A.K.; Chang, C.K.; Lee, Y.D. Molecularly imprinted polymeric film on semiconductor nanoparticles: Analyte detection by quantum dot photoluminescence. J. Chromatogr. A. 2004, 1027(1-2), 259-262.

[206] Wang, H.; He, Y.; Ji, T.; Yan, .X. Surface molecular imprinting on Mn-doped ZnS quantum dots for room-temperature phosphorescence optosensing of pentachlorophenol in water. Anal. Chem. 2008, 81, 1615-1621.

[207] Ren, X.; Liu, H.; Chen, L. Fluorescent detection of chlorpyrifos using Mn(II)-doped $\mathrm{ZnS}$ quantum dots coated with a molecularly imprinted polymer. Microchim Acta. 2015, 182, 193-200.

[208] Veneckoba, T.; Bezdekova, J.; Tvrdonova, M.; Vlcnovska, M.; Novotna, V.; Neuman, J.; Stossova, A.; Kanicky, V.; Adam, V.; Vaculovicova, M.; Vaculovic, T. CdS quantum 
dots-based immunoassay combined with particle imprinted polymer technology and laser ablation ICP-MS as a versatile tool for protein detection. Scientific Reports. 2019, 9, 11840. [209] Wang, J.; Dai, J.; Xu, Y.; Dai, X.; Zhang, Y.; Shi, W.; Sellergren, B.; Pan, C. Molecularly imprinted fluorescent test strip for direct, rapid, and visual dopamine detection in tiny amount of biofluid. Small. 2019, 15, 1803912.

[210] Shariati, R.; Rezaei, B.; Jamei, H.R.; Ensafi, A.A. Application of coated green source carbon dots with silica molecularly imprinted polymers as a fluorescence probe for selective and sensitive determination of phenobarbital. Talanta. 2019, 194, 143-149.

[211] Qin, Y.T.; Feng, Y.S.; Ma, Y.J.; He, X.W.; Li, W.Y.; Zhang, Y.K. Tumor-Sensitive Biodegradable Nanoparticles of Molecularly Imprinted Polymer-Stabilized Fluorescent Zeolitic Imidazolate Framework-8 for Targeted Imaging and Drug Delivery. ACS Appl. Mater. Interfaces. 2020, 12(22), 24585-24598.

[212] Mo, G.; He, X.; Zhou, C.; Ya, D.; Feng, J.; Yu, C.; Deng, B. A novel ECL sensor based on a boronate affinity molecular imprinting technique and functionalized SiO2@CQDs/AuNPs/MPBA nanocomposites for sensitive determination of alphafetoprotein. Biosens. Bioelectron. 2019, 126, 558-564.

[213] Chmangui, A.; Driss, M.R.; Touil, S.; Bermejo-Barrera, P.; Bouabdallah, S.; MoredaPiñeiro, A. Aflatoxins screening in non-dairy beverages by Mn-doped ZnS quantum dots - Molecularly imprinted polymer fluorescent probe. Talanta. 2019, 199, 65-71.

[214] Hatamluyi, B.; Rezayi, M.; Beheshti, H.R.; Boroushaki, M..T. Ultra-sensitive molecularly imprinted electrochemical sensor for patulin detection based on a novel assembling strategy using Au@Cu-MOF/N-GQDs. Sens. Actuat. B-Chem. 2020, 318, 128219. [215] Feng, J.; Tao, Y.; Shen, X.; Jin, H.; Zhou, T.; Zhou, Y.; Hu, L.; Luo, D.; Mei, S.; Lee, Y.I. Highly sensitive and selective fluorescent sensor for tetrabromobisphenol-A in electronic waste samples using molecularly imprinted polymer coated quantum dots. Microchem. J. 2019, 144, 93-101.

[216] Yao, J.; Chen, M.; Li,N.; Liu, C.; Yang, M. Experimental and theoretical studies of a novel electrochemical sensor based on molecularly imprinted polymer and B, N, FCQDs/AgNPs for enhanced specific identification and dual signal amplification in highly selective and ultra-trace bisphenol $\mathrm{S}$ determination in plastic products. Anal. Chim. Acta. 2019, 1066, 36-48.

[217] Matsui, J.; Akamatsu, K.; Nishiguchi, S.; Miyoshi, D.; Nawafune, H.; Tamaki, K.; Sugimoto, N. Composite of Au nanoparticles and molecularly imprinted polymer as a sensing material. Anal. Chem. 2004, 76, 1310-1315.

[218] Wu, M.; Deng, H.; Fan, Y.; Hu, Y.; Guo, Y.; Xie, L. Rapid colorimetric detection of cartap residues by AgNP sensor with magnetic molecularly imprinted microspheres as recognition elements. 2018, Molecules 23, 1442-1443.

[219] Zhao, B.; Feng, S.; Hu, Y.; Wang, S.; Lu, X. Rapid determination of atrazine in apple juice using molecularly imprinted polymers coupled with gold nanoparticlescolorimetric/SERS dual chemosensor. Food Chem. 2019, 276, 366-375.

[220] Feng, S.; Hu, Y.; Ma, L.; Lu, X. Development of molecularly imprinted polymerssurface-enhanced raman spectroscopy/colorimetric dual sensor for determination of chlorpyrifos in apple juice. Sensors \& Actuators B. 2017, 241, 750-757.

[221] Kong, Q.; Wang, Y.; Zhang, L.; Ge, S.; Yu, J. A novel microfluidic paper-based colorimetric sensor based on molecularly imprinted polymer membranes for highly selective and sensitive detection of bisphenol A. Sensors \& Actuators B. 2017, 243, 130-136. 
[222] McNiven, S.; Kato, M.; Levi, R.; Yano, K.; Karube, I. Chloramphenicol sensor based on an in situ imprinted polymer. Analytica Chimica Acta. 1998, 365, 69-74.

[223] Nicholls, C.; Karim, K.; Piletsky, S.; Saini, S.; Setford, S. Displacement imprinted polymer receptor analysis (DIPRA) for chlorophenolic contaminants in drinking water and packaging materials. Biosensors and Bioelectronics. 2006, 21, 1171-1177.

[224] Li, C.; Ngai, M. H.; Reddy, K. K.; Leong, S. C. Y.; Tong, Y. W.; Chai, C. L. L. A fluorescence-displacement assay using molecularly imprinted polymers for the visual, rapid, and sensitive detection of the algal metabolites, geosim and 2-methylisoborneol. Analytica Chimica Acta. 2019, 1066, 121-130.

[225] Haupt, K.; Mayes, A. G.; Mosbach, K. Herbicide assay using an imprinted polymerbased system analogous to competitive fluoroimmunoassays. Anal. Chem. 1998, 70, 39363939.

[226] Piletsky, S. A.; Terpetschnig, E.; Andersson, H. S.; Nicholls, I. A.; Wolfbeis, O. S. Application of non-specific fluorescent dyes for monitoring enatio-selective ligand binding to molecularly imprinted polymers. J Anal Chem. 1999, 364, 512-516.

[227] Greene, N. T.; Shimizu, K. D. Colorimetric molecularly imprinted polymer sensor array using dye displacement. J. Am. Chem. Soc. 2005, 127, 5695-5700.

[228] Silverio, O. V.; So, R. C.; Elnar, K. J. S.; Malapit, C. A.; Nepomuceno, M. C. M. Development of dieldrin, endosulfan, and hexachlorobenzene-imprinted polymers for dye-displacement array sensing. J. Appl. Polym. Sci. 2017, 134(2), 44401.

[229] Mattsson, L.; Xu, J.; Preininger, C.; Tse Sum Bui, B.; Haupt, K. Competitive fluorescent pseudo-immunoassay exploiting molecularly imprinted polymers for the detection of biogenic amines in fish matrix. Talanta. 2018, 181, 190-196.

[230] Lowdon, J.W.; Eersels, K.; Rogosic, R.; Heidt, B.; Diliën, H.; Steen Redeker, E.; Peeters, M.; van Grinsven, B.; Cleij, T.J. Substrate displacement colorimetry for the detection of diarylethylamines. Sens. Actuat. B-Chem. 2019, 282, 137-144.

[231] Piletsky, S.A.; Piletska, E.V.; Chen, B.; Karim, K.; Weston, D.; Barrett, G.; Lowe, P.; Turner, A.P.F. Chemical Grafting of Molecularly Imprinted Homopolymers to the Surface of Microplates. Application of Artificial Adrenergic Receptor in Enzyme-Linked Assay for $\beta$-Agonists Determination. Anal. Chem. 2000, 72(18), 4381-4385.

[232] Surugiu, I.; Danielsson, B.; Ye, L.; Mosbach, K.; Haupt, K. Chemiluminescence Imaging ELISA Using an Imprinted Polymer as the Recognition Element Instead of an Antibody. Anal. Chem. 2001, 73(3), 487-491.

[233] Piletsky, S.A.; Piletska, E.V.; Bossi, A.; Karim, K.; Lowe P.; Turner, A.P.F. Substitution of antibodies and receptors with molecularly imprinted polymers in enzyme-linked and fluorescent assays. Biosens. Bioelectron. 2001, 16(9-12), 701-707.

[234] He, Y.; Hong, S.; Wang, M.; Abd El-Aty, A.M.; Wang, J.; Hacimuftuoglu, A.; Khan, M.; She, Y. Development of fluorescent lateral flow test strips based on an electrospun molecularly imprinted membrane for detection of triazophos residues in tap water. New J. Chem. 2020, 44, 6026-6036.

[235] Akbulut, Y.; Zengin, A. A molecularly imprinted whatman paper for clinical detection of propranolol. Sens. Actuat. B-Chem. 2020, 304, 127276.

[236] Capoferi, D.; Alvarez-Diduk, R.; Del Carlo, M.; Compagnone, D.; Merkoçi, A. Electrochromic Molecular Imprinting Sensor for Visual and Smartphone-Based Detections. Anal. Chem. 2018, 90(9), 5850-5856.

[237] Sergeyeva, T.; Yarynka, D.; Piletska, E.; Linnik, R.; Zaporozhets, O.; Brovko, O.; Piletsky, S.; El'skaya, A. Development of a smartphone-based biomimetic sensor for 
aflatoxin B1 detection using molecularly imprinted polymer membranes. Talanta. 2019, 201, 204-210.

[238] Savoy, M. C.; Woo, P.M.; Ulrich, P.; Tarres, A.; Mottier, P.; Desmarchelier, A. Determination of 14 aminoglycosides by LC-MS/MS using molecularly imprinted polymer solid phase extraction for clean-up. Food Addit. Contam. A. 2018, 35(4), 675-686.

[239] Turner, N. W.; Jeans, C. W.; Brain, K. R.; Allender, C. J.; Hlady, V.; Britt, D. W. From 3D to 2D: a review of the molecular imprinting of proteins. Biotechnol. Prog. 2006, 22(6), 1474-1489.

[240] Yang, X.; Yang, S.; Yang, S.; Hu, J.; Tan, X.; Wang, X. Effect of pH, ionic strength and temperature on sorption of $\mathrm{Pb}$ (II) on NKF-6 zeolite studied by batch technique. Chem. Eng. J. 2011, 168(1), 86-93.

[241] Canfarotta, F.; Smolinska Kempisty, K.; Piletsky, S. Replacement of antibodies in pseudo-ELISAs: molecularly imprinted nanoparticles for vancomycin detection. Methods Mol. Biol. 2017, 1575, 389-398.

[242] D'Aurelio, R.; Chianella, I.; Goode, J. A.; Tothill, I.E. Molecularly imprinted nanoparticles based sensor for cocaine detection. Biosensors. 2020, 10(3), 22.

[243] Mazzotta, E.; Turco, A.; Chianella, I.; Guerreiro, A.; Piletsky, S.A.; Malitesta, C. Solidphase synthesis of electroactive nanoparticles of molecularly imprinted polymers. A novel platform for indirect electrochemical sensing applications. Sens. Actuators B Chem. 2016, 229, 174-180.

[244] Canfarotta, F.; Czulak, J.; Betlem, K.; Sachdeva, A.; Eersels, K.; Van Grinsven, B.; Cleij, T. J.; Peeters, M. A novel thermal detection method based on molecularly imprinted nanoparticles as recognition elements. Nanoscale, 2018, 10(4), 2081-2089.

[245] Betlem, K.; Canfarotta, F.; Raumbault, R.; Banks, C.E.; Eersels, K.; van Grinsven, B.; Cleij, T.J.; Crapnell, R.; Hudson, A.; Peeters, M.; Thermistors coated with molecularly imprinted nanoparticles for the electrical detection of peptides and proteins. Analyst 2020. [246] Yeo, C.; Kaushal, S.; Yeo, D.; Enteric involvement of coronaviruses: is faecal-oral transmission of SARS-CoV-2 possible? The lancet Gastroenterology \& hepatology 2020, 5(4), 335-337.

[247] Cecchini, A.; Raffa, V.; Canfarotta, F.; Signore, G.; Piletsky, S.; MacDonald, M. P.; Cuschieri, A. In vivo recognition of human vascular endothelial growth factor by molecularly imprinted polymers. Nano letters. 2017, 17(4), 2307-2312. 


\section{Chapter 3}




\section{Surface grafted polymers preface:}

The literature review conducted in the previous chapter, highlighted the use of multiple platforms for the detection of small molecules. Of these, one of the promising technologies was identified to be the so-called "Heat Transfer Method" (HTM), offering a thermal detection method that translate a binding event at the surface of an imprinted polymer into a tangible change in thermal resistance. References were found for this readout platform being used for the rapid detection of small molecules (e.g. neurotransmitters), bacteria (e.g. E Coli) and mutations in DNA. Therefore, the decision was made to adept the readout platform towards the detection of a different class of compound, pushing the potential usage of the readout strategy further.

The template molecule for the imprinted layers was selected based on previous research, targeting New Psychoactive Substances (NPS). 2-Methoxphendine was identified as the target of choice, being an emerging NPS at the time and being commonly found mixed with ketamine and PCP samples. These kind of compounds have proven to be a plague in their own right, spanning multiple compound classes and proving tricky to identify. It therefore made sense to develop a platform capable of potentially identifying an NPS and showcase how MIPs could be used as a tool in the battle against these illicit substances.

Unlike previous research conducted with the sensory platform, a different approach was selected for the deposition of the polymeric receptor layers. In prior literature MIP particles were prepared by bulk polymerization; yielding an imprinted polymeric powder that could be adhered to an aluminium substrate and placed inside a flow cell for the analysis of binding capabilities. Though this has been proven an efficient way of MIP deposition, the method is relatively heterogeneous across the deposited layer and from sample to sample. In an attempt to remedy this, a surface grafting approach was identified, functionalizing an aluminum substrate by hydroxylation, attaching a silylated linker molecule with allyl functionalities, and directly building an imprinted polymeric receptor layer upon it. 


\section{Research paper: Surface Grafted Molecularly Imprinted Polymeric receptor layers for Thermal Detection of the New Psychoactive Substance 2- methoxphenidine}

Joseph W. Lowdon ${ }^{1}$, Kasper Eersels ${ }^{1}$, Renato Rogosic ${ }^{1}$, Tine Boonen ${ }^{1}$, Benjamin Heidt ${ }^{1}$, Hanne Diliën ${ }^{1}$, Bart van Grinsven ${ }^{1}$, Thomas J. Cleij ${ }^{1}$

${ }^{1}$ Faculty of Science and Engineering, Maastricht University, PO Box 616, 6200 MD, Maastricht, the Netherlands 


\begin{abstract}
The use of Molecularly Imprinted Polymers (MIPs) as recognition elements in biosensing devices has increased over the past decade, with MIP-based receptor layers being combined with impedance, quartz crystal microbalance (QCM) and thermal readout methods such as the so-called "Heat-Transfer Method" (HTM). One of the main challenges in MIP-based biosensing is the production of uniformly covered, reproducible sensing layers. The potential of direct surface grafting of molecularly imprinted polymeric receptor layers (MIP-RL) to aluminum substrates was investigated in this work, offering an alternative more elegant methodology of MIP deposition opposed to previous methods introduced. Polished aluminum plates were hydroxylated and further silylated, thereby introducing functionality to the plates allowing for direct polymer grafting to the substrate surface. Various polymer compositions were studied, identifying the optimum composition for direct surface grafting and the detection of the New Psychoactive Substance (NPS) known as 2methoxphenidine (2-MXP). Evaluation using HTM for each grafted MIP-RL determined the imprinting effect, selectivity and sensitivity of the polymeric layers, with a direct comparison being drawn to previously optimized MIP particles that have been deposited by means of a polydimethylsiloxane (PDMS) stamp being pressed into an adhesive polyvinyl chloride (PVC) layer. The results summarized in this study highlight the reproducibility, improved limit of detection (LoD), and feasibility of directly grafting polymeric layers to substrates, offering a more reliable tool in the routine analysis of samples.
\end{abstract}

Keywords: Molecularly Imprinted Polymer, Surface grafting, Grafted receptor layers, Heat-transfer method, New Psychoactive Substance, 2methoxphenidine 


\section{Introduction}

New Psychoactive Substances (NPS), formally known as "designer drugs" have been described as a plague of the modern age [1]. NPS filled the void created by legislation that prohibited classical drug distribution and use, as they share key specific psychoactive effects with classical illicit compounds without the actual prohibited chemical structure [2]. Although legislation more recently halted distribution and use of NPS within the European Union [3], identification, detection and analysis of NPS remain an issue. Hundreds of NPS compounds already exist with chemical architectures that are currently unfamiliar and indistinguishable from the more classical compounds, with more being synthesized every year [4].

Prevalent since 2013, 2-methoxphendine (2-MXP) is an NPS that interacts with NMDA receptors within the brain giving a dissociative 'high' that is comparable to diphenidine, ketamine and Phencyclidine (PCP). 2-MXP is commonly found 'cut' with these compounds (Figure 1), adding complexity to drug sample analysis that commonly involves highly specialized, expensive techniques such as HPLC, LC-MS, and GC-MS as the gold standard for sample analysis [5, 6]. The primary source of information on the prevalence of 2-MXP comes from the analysis of cadavers, with little information being available on 2-MXP in seized drug samples. To this end, cases where 2-MXP has been linked to fatalities is increasing, with reports from multiple countries confirming the presence of 2-MXP in autopsies [7]. These cases also indicate that other substances of abuse are also present, making the need for a selective fast detection method even more necessary [8]. Molecularly imprinted polymers (MIPs) may offer a way of selectively targeting NPS, in this case 2-MXP, allowing for greater ease of sample analysis.

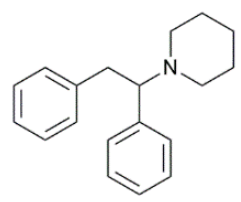

(a)

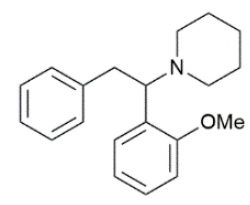

(b)<smiles>CNC1(c2ccccc2Cl)CCCCC1=O</smiles>

(c)<smiles>c1ccc(C2(N3CCCCC3)CCCCC2)cc1</smiles>

(d)

Figure 1. Chemical structures of (a) Diphenidine, (b) 2-MXP, (c) ketamine and (d) PCP 
The field of chemically engineering polymers to contain nanocavities that offer uniquely specific binding towards a target analyte has seen great developments over the last decade [9]. MIPs offer recognition of a target substrate on a similar level to that of enzymes while demonstrating a higher robustness towards harsh physical conditions such as high temperatures or aggressive changes in $\mathrm{pH}$ [10]. Highly specific receptor pockets are generated during the creation of a MIP. Generally, polymerization is conducted in the presence of a target template molecule, crosslinking the polymeric network around the morphology and functionalities of the template [11]. MIP characteristics can be tailored by making alterations in monomer, functional crosslinker and porogen ratios, enhancing the imprinting effect and selectivity of the receptor [12-15]. The ways in which MIPs are synthesized has become more refined over the years, with the more traditional free radical bulk polymerization being usurped by the more elegant synthesis of nanoparticles [16]. This more sophisticated approach yields particles with greater homogeneity in particle size, shape and binding affinity towards a chosen target [17]. These key features, alongside ease of synthesis and being inexpensive, make MIPs ideal synthetic receptors for incorporation into biomimetic sensing platforms for an array of molecular and biological targets [18].

Although the synthesis of these synthetic receptors has developed tremendously, studies aimed at optimizing the methods of integrating them as receptors in biosensing platforms remain relatively unexplored. Readout methods that utilized MIPs as a recognition element include Quartz Crystal Microbalance (QCM), impedance, and more recently the "Heat Transfer Method (HTM)" [19-25]. Measuring the thermal resistance across the receptor layer at the phase boundary between solid and liquid, the HTM allows the binding properties of a receptor layer to be expressed as a change in thermal transfer resistance $[26,27]$. This technique has shown promise in both the detection of peptides and small molecules alike, with MIP particle deposited onto aluminum substrates forming receptor layers.

Two main particle deposition approaches have been extensively used up until now, one of which concerns pressing MIP particles onto a substrate that has previously been coated with a polyvinyl chloride (PVC) adhesive layer. The layer is heated to a temperature above its glass transition temperature to let the particles sink into the layer when force is applied [28-32]. The other method consists of mixing fine MIP particles with e.g. graphene ink, allowing for the direct printing of receptor layers onto surfaces, creating screen-printed electrodes (SPE) [33]. Both methods have their merits and disadvantages, with stamping MIPs being fast and yielding a receptor layer that mimics the 
sensitivity of the original 'free' particles. However, the approach is highly irreproducible, as particles are deposited heterogeneously across the surface of both the stamp and the resulting MIP-coated substrate. Screen-printing allows for greater control over the distribution of particles in a more defined manner, yet sensitivity of the receptor layer suffers due to the encapsulation of the receptor particles in ink. Growing polymeric receptor layers directly on a functionalized substrate could potentially overcome such drawbacks.

Surface functionalization is a powerful tool that allows the coupling of molecules directly to a substrate, providing a robust chemical linkage [34]. This process has been exploited in both the fields of biology and chemistry, offering a multitude of applications from the binding of DNA to substrates, to the modification of a substrate to become more hydrophobic [35]. Many of these approaches have been applied to functionalizing surfaces with MIPs, with atom transfer radical polymerization, and controlled radical polymerization (CRP) being just a couple of techniques applied in the field [36-38]. As of yet, this process has not been used for the direct growth of molecularly imprinted polymeric receptor layers (MIP-RL), offering an alternative to current receptor layer deposition procedures (Figure 2). In this study, a method inspired from previous work in the field of surface functionalization is proposed, functionalizing aluminum substrates by means of hydroxylation and silylation in order for the direct growth of MIP-RL for direct implementation into a HTM setup.

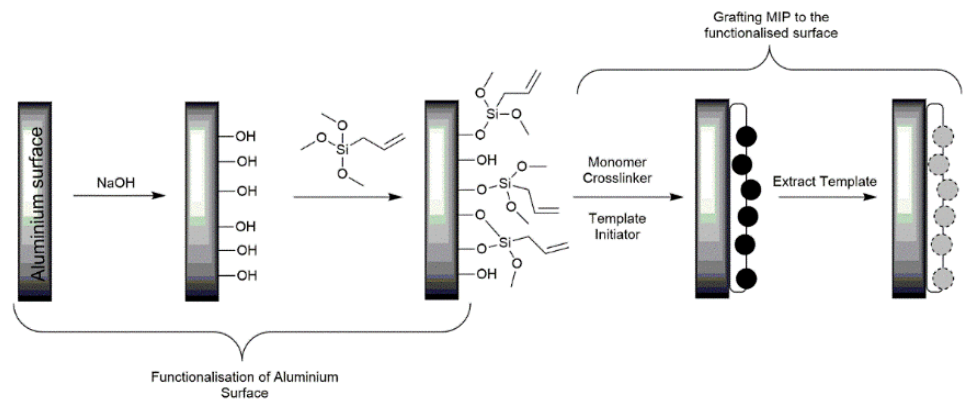

Figure 2. Schematic for the functionalization of aluminum plates, followed by the grafting and extraction of molecularly imprinted polymers directly on the functional substrate.

HTM analysis was used to demonstrate the reproducibility of the direct grafting method of MIP-RL, analyzing the homogeneity of the samples and optimizing receptor layers towards the chosen 2-MXP target. Selectivity and sensitivity of the resulting sensor was assessed, with a direct comparison 
drawn against previous deposition methods, demonstrating the merits of the proposed approach. MIP-RL sensitivity and MIP-RL thickness have demonstrated to be correlated, building a relationship between the thickness of the receptor layer and the sensitivity achieved, with the selectivity of the MIP-RLs also reported. The resulting LoD was compared with biologically relevant concentrations, evaluating the applicability of the sensing platform with the MIP-RL. Ultimately, the combination of MIP-RL with HTM illustrates the potential for reproducibly creating a new method of selective drug detection and analysis. Therefore an early warning system has the potential of being developed, by offering a new rapid means of analyzing seized drug samples for harmful NPS such as 2-MXP. Thus, the prevalence of this NPS can truly be grasped with more than the analysis of cadavers as reference, but instead this approach offers an applicable method of analyzing real world samples in a bid to reduce harm inflicted by 2-MXP. 


\section{Materials and methods}

\section{Chemicals and reagents}

Prior to polymerization, stabilizers were removed from the functional and crosslinking monomers by passing the solutions over a column packed with alumina. All chemicals and solvents were obtained from Sigma Aldrich (Zwijndrecht, the Netherlands). All solutions were prepared with deionized water of resistivity of $18.2 \mathrm{M} \Omega \mathrm{cm}^{-1}$ or with phosphate buffered saline (PBS) solutions. Polydimethylsiloxane (PDMS) stamps were made with the Sylgard 184 elastomer kit from Mavom NV (Kontich, Belgium). Aluminum chips were purchased at Brico NV (Korbeek-Lo, Belgium) and cut to the desired dimensions.

\section{Synthesis of bulk molecularly imprinted polymers}

Bulk MIPs were synthesised according to the procedure previously described..$^{33}$ In short, a mixture of the functional monomer methacrylic acid (MAA $1.02 \mathrm{mmol}$ ), crosslinker molecule ethylene glycol dimethacrylate (EGDMA $1.7 \mathrm{mmol}$ ), and initiator azobisisobutyronitril (AIBN $0.30 \mathrm{mmol}$ ) in dimethyl sulfoxide (DMSO) together with the 2-MXP $(0.17 \mathrm{mmol})$ was prepared. The mixture was purged with $\mathrm{N}_{2}$ before the initiation of the polymerization. Polymerization was performed by heating the mixture up to $65^{\circ} \mathrm{C}$ for $12 \mathrm{~h}$, allowing for full completion of the reaction. The MIP was milled seven times using a Fritsch Planetary Micro Mill Pulverisette 7 premium line (700 rpm, 5 minutes, $10 \mathrm{~mm}$ balls). After milling, the particles were sieved at $1.0 \mathrm{~mm}$ amplitude using a Fritsch Analysette 3 for 4 hours or until sufficient amount of polymer was on the collection plate to achieve microparticles with sizes smaller than $100 \mu \mathrm{m}$. Finally, the template molecule was removed from the MIP powders by continuous Soxhlet extraction with a 1:10 mixture of acetic acid and methanol for $12 \mathrm{~h}$, followed by further extraction with pure methanol for a further $12 \mathrm{~h}$ and drying of the particles at $65^{\circ} \mathrm{C}$ overnight. A reference non-imprinted polymer (NIP) was also prepared in parallel

\section{Deposition of bulk MIP particles}

Polished aluminum plates were cut to obtain chips with the desired dimensions $\left(10 \times 10 \times 5 \mathrm{~mm}^{2}\right)$. To immobilize MIP particles, a $100 \mathrm{~nm}$ polyvinyl chloride (PVC) adhesive layer ( $0.35 \mathrm{wt} \%$ PVC dissolved in tetrahydrofuran) was deposited on the chip by spin coating (3000 rpm for 60 seconds with an acceleration of $1100 \mathrm{rpm} / \mathrm{s}$ ). MIP and NIP particles were stamped into this layer using a PDMS substrate that was covered with a monolayer of polymer 
particles. The PVC layer was heated for 2 hours at a temperature above its glass transition temperature $\left(100{ }^{\circ} \mathrm{C}\right)$ allowing the beads to sink into the polymer layer. The samples were allowed to cool prior to thermal measurements and any unbound particles were washed off with distilled water. Particle distribution was determined using a Keyence VHX-5000 Digital microscope, with further surface analysis being conducted with a Scanning Electron Microscope (SEM).

\section{Hydroxylation and silylation of aluminum plates}

Polished aluminum plates $\left(10 \times 10 \times 5 \mathrm{~mm}^{2}\right)$ were immersed in sodium hydroxide $(20 \% \mathrm{w} / \mathrm{v})$ and ultrasonicated for 10 minutes, producing highly tarnished aluminum surfaces. The plates proceeded to be washed with DI water, before being dried by gentle dabbing with a paper towel and being left to air dry for 3 hours. The hydroxylated aluminum was further functionalized by means of silylation. Where the hydroxylated plates were placed into a beaker containing allyltrimethoxysilane in toluene $(10 \% \mathrm{v} / \mathrm{v})$, and were heated at $65{ }^{\circ} \mathrm{C}$ under continuous stirring for 2 hours. Following this, the plates were removed from the mixture, washed with methanol and left to air dry overnight. Both hydroxylation and siloxane coupling was confirmed by FTIR of the tarnished surface (Supplementary Information S1).

\section{Surface grafting of molecularly imprinted polymeric receptor layers}

The protocol of surface grafting MIP-RL was optimized by altering the ratios of monomer, crosslinker and porogen. To this extent, the optimized synthesis is described for the MIP-RL with the highest affinity towards the target, 2-MXP (see Supplementary Information Table S1 for all ratios tested). A mixture consisting of MAA (11.90 mmol, $1 \mathrm{~mL}$ ), EGDMA (20.18 mmol, $4 \mathrm{~mL}$ ), 2-MXP ( $0.67 \mathrm{mmol}, 200 \mathrm{mg}$ ) in $20 \mathrm{~mL}$ toluene was prepared, and degassed using $\mathrm{N}_{2}$ for 5 minutes. Once degassed the solution was poured into a $250 \mathrm{~mL}$ beaker (diameter $80 \mathrm{~mm}$ ), where a stirrer bar (length $30 \mathrm{~mm}$ ) and 5 silylated aluminum plates were placed. Once added, the solution was stirred at $250 \mathrm{rpm}$, before adding AIBN (200 mg) and heating the stirred solution to $65^{\circ} \mathrm{C}$ to initiate polymerization. The mixture was left to stir for 90 minutes, before removing the now polymer-coated aluminum plates and washing with methanol. To extract the 2-MXP from the polymer coated plates, Soxhlet extraction was undertaken for 6 hours using methanol and acetic acid $(10: 1 \mathrm{v} / \mathrm{v})$, and for a further 6 hours using pure methanol. To monitor the extraction of the 2-MXP from the polymer, UV-spectrometer readings of the filtrate were taken until an absorbance value at $\lambda=278 \mathrm{~nm}$ was no longer observed. Thus, a molecularly imprinted polymeric layer was deposited upon the functionalized aluminum 
plate. A surface grafted non-imprinted polymeric receptor layer (NIP-RL) was prepared in parallel that was synthesized in the absence of 2-MXP. Film thickness and sample homogeneity was determined using a Keyence VHX5000 Digital microscope, with further surface analysis being conducted with a Scanning Electron Microscope (SEM). For each parameter, at least three samples at three different positions were analyzed and a mean film thickness was reported.

\section{Sensing setup}

The thermal detection platform is described thoroughly in previous work. ${ }^{21}$ Functionalized chips were pressed mechanically with their backside onto a copper block serving as a heat provider. The temperature of the copper underneath the sample, $\mathrm{T}_{1}$, was monitored by a K-type thermocouple (TC Direct). This information was fed into a temperature control unit that stringently controlled $\mathrm{T} 1$ by modifying the voltage over the power resistor (Farnell, Utrecht, The Netherlands) that heats the copper, using a softwarebased (Labview, National Instruments, Austin, TX, United States) Proportional-Integral-Derivative (PID) controller $(P=1, I=8, D=0)$. The functionalized side of the chip faced a PolyEther Ether Ketone (PEEK) flow cell which was sealed with an O-ring to avoid leakage, defining a contact area of $28 \mathrm{~mm} 2$ and an inner volume of $110 \mu \mathrm{L}$. The flow cell is connected to a tubing system, allowing to exchange liquids in a controlled and automated fashion by means of a syringe pump. The temperature of the liquid inside the flow cell, $\mathrm{T}_{2}$, was measured by a second thermocouple placed $1 \mathrm{~mm}$ above the chip. For each rebinding measurement, the signal was stabilized in PBS that was used as a background solvent for the measurements. The concentration of the target or analogue inside the flow was gradually increased $(50 \mathrm{nM}-2.25 \mu \mathrm{M})$. The signal was allowed to stabilize for $20 \mathrm{~min}$ between subsequent additions. Data was analyzed by monitoring the decrease in $\mathrm{T}_{2}$ after each addition (heat transfer method or $\mathrm{HTM}$ ) while maintaining $\mathrm{T}_{1}$ at a constant $37.00^{\circ} \mathrm{C}$. 


\section{Results and Discussion}

\section{Binding characteristics of MIPs immobilized by stamping}
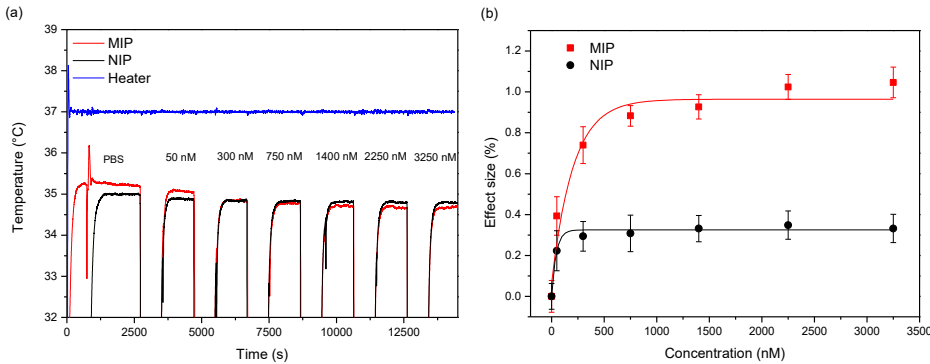

Figure 3. HTM analysis of PDMS stamped MIPs, (a) Temperature profile of the flow cells for both the MIP and NIP after infusions of varying concentrations of aqueous 2-MXP, (b) Dose response calculated from mean values at each stabilization point after each infusion. (Error bars calculated using the internal error / noise of the signal)

To analyze the capability of the MIP to bind 2-MXP, aluminum chips were coated with both MIP and NIP particles immobilized by stamping into an adhesive PVC layer and analyzed using the HTM (Figure 3a). MIP and NIP channels were ran simultaneously in parallel flow cells. The observed differences in the baseline temperature of both flow cells can be attributed to the specific characteristics of each flow cell. The temperature inside the flow cell for the MIP can be seen to decrease at a constant input temperature as an increased concentration of 2-MXP is introduced, indicating that the thermal resistance of the MIP changes in response to the presence of the target in the flow cell. A similar effect can be observed for the NIP at lower concentrations, with the effect diminishing as higher concentrations are reached. These findings are in line with previous work analyzing both neurotransmitters and vitamin $\mathrm{K}$ with the thermal readout setup [32]. The data of this experiment are summarized in a corresponding dose-response curve that allows for an accurate comparison of the data sets for both MIP and NIP respectively (Figure $3 b)$. The effect size was normalized by dividing the observed temperature decrease for each concentration by the average baseline temperature (Equation $1)$.

Equation 1: $\quad$ Effect size $(\%)=\frac{\Delta T_{t}(\mathrm{t}=\mathrm{c})}{T_{t}(t=0)} \times 100$ 
The data was fit using OriginPro8 (OriginLabs Corporation, Northampton, MA, United States) using an allometric $\left(y=a^{x}\right)$ dose-response fit for both the MIP (red curve, $\mathrm{R}^{2}=0.9667$ ) and NIP (black curve, $\mathrm{R}^{2}=0.8805$ ). The limit-ofdetection $(\mathrm{LoD})$ was calculated as the value that corresponds to three times the maximum amount of noise on the signal throughout the measurement and its intercept with the linear part of the red curve which predicts an LoD of $100 \mathrm{nM}$ ( $3 \sigma$ method). Mean values and error bars were calculated over a range of 700 seconds after each concentration infusion. The calculated LoD lies below the point of differentiation between the MIP and NIP signals, rendering sample analysis in this concentration range unreliable. At higher concentrations (above $300 \mathrm{nM}$ ), there is a defined difference between the two signals allowing for more confidence within the readings taken. In a bid to create a MIP with a more reliable $\mathrm{LoD}$ at lower concentrations (sharper differentiation between MIP and NIP and a broader dynamic range) a more sophisticated method of MIP deposition is required.

\section{Analysis of thickness and homogeneity of MIP-RL deposition}

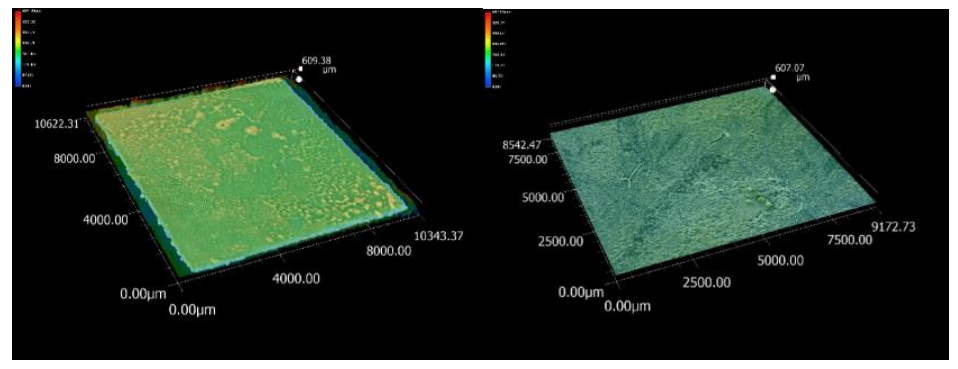

Figure 4. Analysis of the overall surface thickness of the surface grafted polymer, (a) a sample from the initial batch synthesised and (b) a sample from the second batch synthesised

The surface of the polymeric grafted aluminum plates was analyzed using a 3D microscope to determine the overall thickness of the layer across the substrate, showing homogeneity of the polymer coverage. This analysis was conducted for two separate batches to analyze inter-batch surface thickness variance, demonstrating that the method of surface grafting gave an overall consistent grafted polymer thickness (Figure 4). On average, the overall thickness of the coated aluminum substrates was $108.17 \mu \mathrm{m}(608.23 \mu \mathrm{m}$ including the plate height), showing minimal variance between each sample. This demonstrates the reproducibility of the method, compared to that of the stamping method where particle distribution across the surface is harder to 
control (Supplementary Information Figure S2). Intra-sample polymer coverage was more heterogeneous, with greater variances across the surface of the MIP layer.

\section{Effectiveness of MIP-RLs}

Table 1. A brief summary of results collected for each composition of polymer in regards to HTM analysis

\begin{tabular}{c|cc} 
MIP & $\begin{array}{c}\text { Compositional } \\
\text { reasoning }\end{array}$ & $\begin{array}{c}\text { Brief analysis of results } \\
\text { obtained }\end{array}$ \\
\hline $\mathbf{0 1}$ & $\begin{array}{c}\text { Ratios obtained from } \\
\text { previous work }\end{array}$ & NIP signal unstable \\
\hline $\mathbf{0 2}$ & $\begin{array}{c}\text { More monomer to } \\
\text { reduce non-specific } \\
\text { binding }\end{array}$ & $\begin{array}{c}\text { Sharp differentiation MIP } \\
\text { and NIP, optimal } \\
\text { dynamic range }\end{array}$ \\
\hline $\mathbf{0 3}$ & $\begin{array}{c}\text { More porogen to } \\
\text { stimulate nanocavity } \\
\text { formation }\end{array}$ & $\begin{array}{c}\text { MIP layer unstable in } \\
\text { water, signal fluctuating }\end{array}$ \\
\hline \multirow{204}{*}{0} & $\begin{array}{c}\text { More porogen to } \\
\text { stimulate nanocavity } \\
\text { formation }\end{array}$ & $\begin{array}{c}\text { Layer more stable than } \\
\text { MIP3 but no MIP/NIP }\end{array}$ \\
& differentiation
\end{tabular}

Functional and crosslinking monomers were selected for the synthesis of the grafted polymers based on previous work [5]. Different MIP batches with different monomer:crosslinker ratios were evaluated using HTM, with MIPRL and NIP-RL readings occurring simultaneously in parallel running flow cells. The data of these measurements were summarized in Table 1 (raw data in Supplementary S3). The imprinting effect (IE) of the MIP-RL's was also determined with MIP-RL02 demonstrating the highest IE $=3.49(\mathrm{C}=25 \mathrm{nM})$, indicating binding of 2-MXP towards the MIP-RL was 3.5 times more effective than that towards the NIP (see Supplementary Table S1). MIP-RL02 provided the most significant results of the compositions tested, not only in terms of analyte binding to the MIP-RL/NIP-RL but in terms of the amount of noise within the signals analyzed. As 2-MXP is introduced into the system, the MIP$\mathrm{RL}$ begins to bind the target analyte, leading to an increase in thermal resistance that can be observed as a decrease of the transmitted temperature 
(Figure 5a). The effect size was calculated for both MIP-RL and NIP-RL (Figure $5 b)$ and used to construct a dose-response curve that demonstrates a clear difference over the entire concentration range. The curves were fit using a dose-response fit in OriginPro8 (OriginLabs Corporation, Northampton, MA, United States) the MIP (red curve, $\mathrm{R}^{2}=0.96404$ ) and NIP (black curve, $\mathrm{R}^{2}$ $=0.74904$ ). The LoD was calculated to be $13 \mathrm{nM}$ using the previously described $3 \sigma$ method. Although the dynamic range only stretches from $10-120 \mathrm{nM}$, the sharp differentiation between MIP-RL and NIP-RL and the improvement of the LoD by nearly an order of magnitude are highly beneficial in terms of analytical application of the sensor. The improved LoD can be attributed to a more stable thermal signal and an improved effect size. The stability of the measurement signal can be understood in terms of homogeneity of the solidliquid interface. The grafting approach yields one homogenous phase boundary for the thermal signal to permeate, whereas the stamping of MIP particle introduces two heterogeneous boundaries. Therefore, the signal from the grafted receptor layers contains less noise as the thermal signal does not have to compensate for multiple changes in heat capacity across multiple materials. The grafted polymeric layers offer greater surface area compared to the previous deposition of polymeric particles, with an increased area of the receptor layer that can potentially bind analyte. These considerations offer validity to the increased effect size demonstrated by the MIP-RL and the ability of the receptor layer to bind 2-MXP.
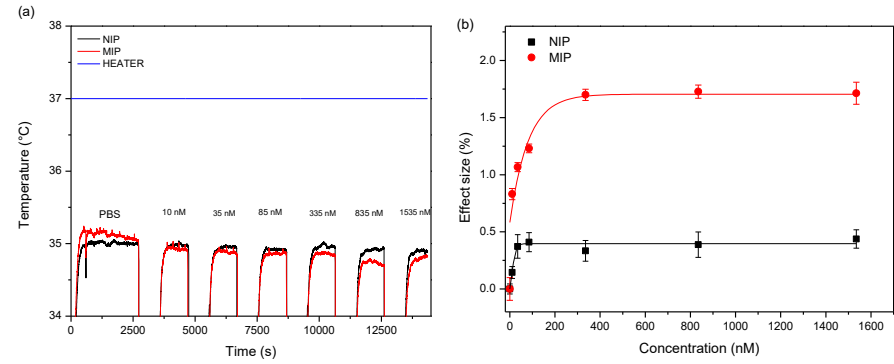

Figure 5. HTM analysis of MIP02, (a) Temperature profile for both the MIP and NIP through the experiment for a series of 7 infusions, (b) the dose response calculated from mean values at each stabilisation point after each infusion. (Error bars calculated using the internal error / noise of the signal)

\section{Reproducibility of MIP-RL}

To demonstrate the reproducibility of surface grafting of MIP-RL for 2-MXP detection, multiple batches of receptor layers were grafted and performance 
comparisons drawn. Rebinding was analyzed using the HTM and the data processed as described in the previous chapters. The results, summarized in Table 2, indicate that the inter-batch variance is minimal with an average LoD of $12.3 \pm 3 \mathrm{nM}$ and average binding capacity of $112 \pm 10 \mathrm{nM}$ being observed. These results confirm and highlight the reproducibility of the polymeric grafting method. Therefore, the noise on the signal can be regarded as the limiting factor in terms of sensitivity. The standard error shown in all HTM measurements is therefore the maximum error on the measurement signal observed in the part of the graph under analysis.

Table 2. Limit of detection and binding capacities for different batches of MIP02 synthesised

\begin{tabular}{c|c|c} 
MIP Batch & LoD (nM) & Binding Capacity (nM) \\
\hline 1 & 13 & 120 \\
2 & 9 & 100 \\
3 & 15 & 115
\end{tabular}

\section{Optimization of receptor layer thickness}

Surface grafting of the polymeric receptor layer was optimized further, controlling layer thickness by varying the amount of time the functionalized aluminum was subjected to the polymerization mixture. Generating a correlation between exposure time to the polymerization mixture, and the thickness of the receptor layer generated (Figure 6a). The culminating graph displayed a linear relationship between the time and layer thickness for MIP $\left(\mathrm{R}^{2}=0.97545\right)$ and NIP $\left(\mathrm{R}^{2}=0.96882\right)$, as the exposure time increased as did the receptor layer thickness until 180 minutes. At this point, the polymerization mixture reached a critical point causing rapid spontaneous polymerization throughout the solution. Formation of a rigid polymer sheet therefore ensued, covering the entirety of the base of the beaker. Loss of porogen throughout the experiment due to evaporation may have been the causality, resulting from constant heating of the porogen at $65{ }^{\circ} \mathrm{C}$ or the heat generated from the polymerization itself. Observed polymer formation occurred more rapidly for the NIP, stemming from the absence of template molecule that can limit the interactions between the reacting radical initiator, crosslinker, and monomer. Therefore polymer formation occurs more steadily for the MIP that is inhibited 
by the presence of 2-MXP, making the overall thickness of the polymeric layer less at each time point.

An optimum receptor layer thickness was found, with each of the layers tested showing a variety of sensitivities towards the exposed concentrations (Figure $6 \mathrm{~b})$. Initially the sensitivity of the receptor layer increases with the thickness of the layer, indicating the recognition of the polymer towards the target analyte is high. The amount of potential binding sites increases with increasing layer thickness, leading to an improved sensitivity of the methodology. However, when the receptor layer thickness reaches $110 \mu \mathrm{m}$, the sensitivity of the layers decrease. The mass and bulk of the polymer is much greater than the target analyte, therefore it takes a greater concentration of analyte to produce a significant response. Therefore, an optimum layer thickness is established between mass transfer of polymeric receptor layer and thermal resistance induced by occupying binding sites at the solid-liquid interface. To confirm this relationship, the corresponding dose response curves were fitted $(\mathrm{y}=$ $\left.A e^{\frac{-x}{t}}+y^{0}\right)$ using OriginPro8. Values of the constant (A) were taken as a representative of each of the curves fitted and plotted against the respective layer thicknesses generating a graphical representation of the relationship between sensitivity and receptor layer thickness (Figure 6c). A polynomial fit was found to represent the data set best $\left(R^{2}=0.96138\right)$, confirming the optimum receptor layer thickness of $110 \mu \mathrm{m}$, and the relationship between sensitivity and receptor layer thickness that was previously theorized. 

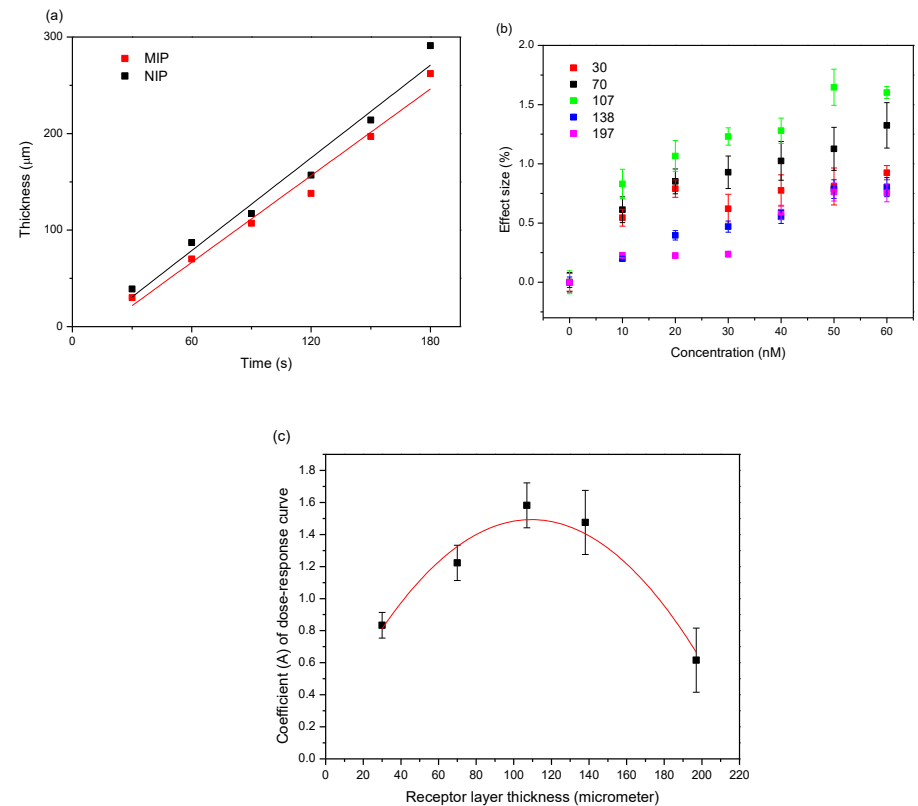

Figure 6. (a) Thickness of MIP/NIP-RL with an increasing exposure time to the polymerization mixture, (b) Dose response curve of each receptor layer thickness when exposed to varying concentrations of 2-MXP during HTM analysis, (c) relationship between receptor layer thickness and the coefficient (A) of the corresponding dose response curve fit $\left(y=A e^{\frac{-x}{t}}+y^{0}\right)$. Error bars were generate from the error calculated in each of the curves exponential fitting.

\section{Selectivity of MIP-RLs}

Selectivity of the MIP-RL was demonstrated by conducting further HTM analysis of the grafted receptor layer samples. Experimental parameters and the procedure remained as per pervious, while MIP-RLs were exposed to analogue molecules and 2-MXP. Competitive analytes were selected based on chemical architecture, similarities in psychoactive effect and if the compound is a known common adulterant that is found "cut" with 2-MXP. To this end, diphenidine (structural analogue of 2-MXP), paracetamol, caffeine, and sucrose (both common adulterants found in drug samples) were selected for analysis (chemical structure in Supplementary Information Figure S4). All competitive analogues were solvated in PBS beforehand, being prepared in the 
same concentration range previously tested with 2-MXP $(10-60 \mathrm{nM})$, therefore allowing a direct comparison to be made between the compounds. As previously stated the temperature response observed for each compound at all concentrations under study was converted into an effect size and plotted against the concentration of analyte, allowing for the construction of a comparative dose-response curve for the MIPs (Figure 7a) and NIPs (Figure $7 \mathrm{~b}$ ). The generated dose response curves correspond to how the stated molecule interacted with the MIP-RL imprinted for 2-MXP, with a dose response for 2-MXP being plotted for a direct comparison.
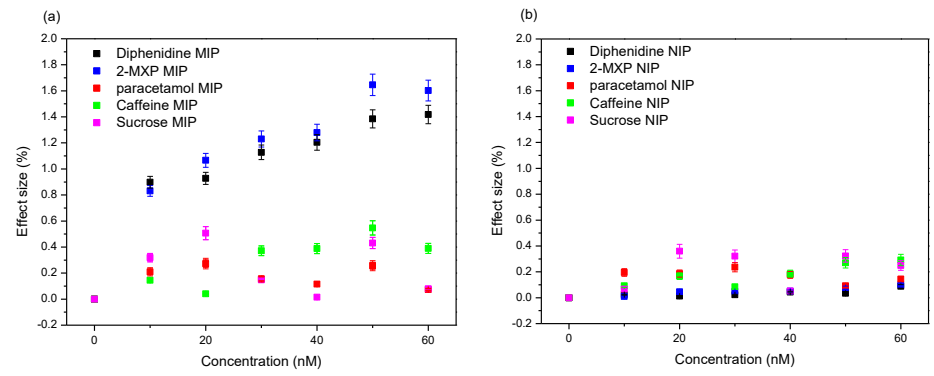

Figure 7. HTM analysis of compounds (caffeine, 2-MXP, diphenidine, sucrose and paracetamol) introduced to the receptor layer inside the flow cell at varying concentrations $(10-60 \mathrm{nM})$ and their corresponding effect size for (a) MIP-RL and (b) NIPRL. (Error bars calculated using the internal error / noise of the signal, and were the average of multiple sample analyzed)

From figure 7a, 2-MXP presents the highest level of binding to the MIP-RL, with diphenidine also demonstrating a high affinity towards the receptor layer. The binding towards the receptor layer of the two compounds is marginally different, with standard deviations from both demonstrating an overlap with each other. This close proximity in binding suggests no significant difference in terms of binding between the two structures. This can be attributed to the diphenidine only differing in structure from 2-MXP due to the presence of a methoxy group on the aryl system. As the methacrylic acid present in the receptor layer primarily interacts with the amine present in both molecules, similar binding behaviour was to be expected. The marginally higher affinity for 2-MXP can therefore be credited to the unique spatial arrangement of 2-MXP, complementing the nanocavities present in the receptor layer in a more defined way.

Differentiation between 2-MXP and the other compounds tested proved easier, with caffeine, paracetamol and sucrose having a diminished effect size in 
comparison to the diarylethylamines. These molecules contain varying functionalities including ones present in 2-MXP, however both the spatial distribution of these analogues as their chemical structures is significantly different from that of the target, resulting in a reduced interaction with the binding pockets in the MIPs. This further illustrates that the imprinting effect is a combination of multiple interactions.

Table 3. Imprinting Effect (IE) for the MIP-RL compared to that of the NIP-RL at C $=0.25$ $\mathrm{nM}$ for the comparison of how the IE differs for each of the compounds tested.

\begin{tabular}{c|c} 
Compound & IE \\
\hline 2-MXP & 27.51 \\
Diphenidine & 23.85 \\
Caffeine & 0.93 \\
Sucrose & 3.07 \\
Paracetamol & 0.15
\end{tabular}

When comparing MIP-RL (Figure 7a) and NIP-RL (Figure 7b) a direct comparison between non-imprinted receptor layer and imprinted receptor layer can be drawn. Table 3 indicating the IE observed by the MIP-RL with respect to NIP-RL for each of the analytes tested. The IE was calculated in much of a similar way to the more classical imprinting factor by taking the effect size of both MIP-RL and NIP-RL at a given concentration and dividing one by the other. Thus, the IE provides information how the MIP-RL functions compared to that of the reference NIP-RL layer. The IE for both 2-MXP and diphenidine are higher than that of the other analytes tested, showing that the MIP-RL has a higher affinity towards these targets. This is a strong indicator of the imprinting effect accomplished in the preparation of the receptor layers, with high selectivity being shown towards the target molecule and a structural analogue. The other analyte structures tested showed low IE values, with some values being indicative of the NIP-RL displaying a higher affinity towards the analyte than the MIP-R. 


\section{Conclusions}

The data presented in this article clearly illustrate the benefits that surface grafting polymeric receptor layers has in the field of sample analysis and elucidation. Straightforward synthetic modification of aluminum plates allowed for the direct grafting of finely tuneable recognition layers, avoiding the need for further deposition methods. This resulted in more homogenously covered substrates and an improved sensitivity in comparison to more traditional approaches of immobilizing MIP particles onto a sensing surface.

This study further illustrates that receptor layer composition is critical in determining the binding characteristics of a synthetic receptor layers. Optimized MIP particles and MIP-RL both contained MAA and EGDMA as constituent elements of the polymeric structure, demonstrating the versatility and applicability of the monomer and functional crosslinker in both situations. Imprinted receptor layers showed higher molecular recognition capabilities compared to their non-imprinted references, indicating the synthetic approach is a viable method of generating imprinted polymeric networks. An observed reduction in LoD was achieved with the grafted MIP-RL, leading to a 10-fold increase in sensitivity compared to that of the stamped MIP particles. A new lower LoD of $13 \mathrm{nM}$ opens up the possibility of trace analysis compared to the previously established LoD range of the MIP particles that sat at $100 \mathrm{nM}$.

The sensitivity of the receptor layers was investigated, building a relationship between layer thickness and sensitivity. The study established the optimum layer thickness, correlating layer thickness with thermal sensitivity. A receptor layer thickness of $110 \mu \mathrm{m}$ proved to be the optimum, with values lower or greater than this having an impeded reduced sensitivity towards detection of the 2-MXP target. The selectivity of the receptor layer towards 2-MXP was also demonstrated with compounds with an array of functionalities and chemical architectures being introduced to the receptor layer. 2-MXP was proven to have the highest binding affinity at lower concentrations, generating the highest effect size out of the compounds tested. This concept was further investigated by comparing the MIP-RL directly with the NIP-RL, generating IE values that allows a numerical value to be related to the higher binding observed by 2-MXP. Thus, demonstrating the imprinting effect relies upon interactions between functionalities and the spatial arrangement of molecules to ensure a dependable level of molecular recognition.

In short, each of the experiments presented demonstrates the versatile nature of polymeric receptors and how they can be exploited in sensing devices. Grafting polymeric layers directly to substrates is shown to yield a polymeric 
layer that has sensitive recognition capabilities and offers an alternative to other deposition methods. Highlighting the benefits of growing a polymeric structure directly on a surface, offering higher reproducibility and greater sensitivity than previously seen when coupled with the HTM.

\section{Acknowledgements}

The authors are grateful for funding through the "Limburg Meet" project funded by the province of Limburg, the Netherlands. Technical support by numerous Maastricht Science Programme undergrad students is greatly appreciated. 


\section{Chapter 3 References}

[1] Castaneto, M. S.; Gorelick, D. A.; Desrosiers, N. A.; Hartman, R. L.; Pirard, S.; Huestis, M. A. Synthetic Cannabinoids: Epidemiology, Pharmacodynamics, and Clinical Implications. Drug Alcohol Depend. 2014, 1, 12-41.

[2] Beharry, S.; Gibbons, S. An Overview of Emerging and New Psychoactive Substances in the United Kingdom. Forensic Sci. Int. 2016, 267, 25.

[3] Geyer, P. M.; Hulme, M. C.; Irving, J. P.; Thompson, P. D.; Ashton, R. N.; Lee, R. J.; Johnson, L.; Marron, J.; Banks, C.E.; Sutcliffe, O. B. Chemical Synthesis, Characterisation and in Vitro and in Vivo Metabolism of the Synthetic Opioid MT-45 and its Newly Identified Fluorinated Analogue 2F-MT-45 with Metabolite Confirmation in Urine Samples From Known Drug Users. Anal. Bioanal. Chem. 2016, 408, 8467.

[4] Helander, A.; Beck, O.; Baeckberg, M. Intoxications by the Dissociative New Psychoactive Substances Diphenidine and Methoxphenidine. Clin. Toxicol. 2015, 53, 446.

[5] Lowdon, J. W.; Alkirkit, M. O.; Mewis, R. E.; Fulton, D.; Banks, C. E.; Sutcliffe, O. B.; Peeters, M. Engineering Molecularly Imprinted Polymers (MIPs) for the Selective Extraction and Quantification of the Novel Pscychoactive Substance (NPS) Methoxphenidine and its Regioisomers. Analyst. 2018, 143, 2002-2007.

[6] Davies, S.; Lee, T.; Ramsey, J.; Dargan, P. I.; Wood, D. M. Risk of Caffeine Toxicity Associated with the Use of 'Legal Highs' (novel psychoactive substances). Eur. J. Clin. Pharmacol. 2012, 68, 435.

[7] Elliott, S.; Sedefov, R.; Evans-Brown, M. Assessing the toxicological significance of new psychoactive substances in fatalities, Drug Test Anal. 2018, 53, 446-453.

[8] Hasegawa, K.; Wurita, A.; Minakata, K.; Gonmori, K.; Nozawa, H.; Yamagishi, I.; Watanabe, K.; Suzuki, O. Postmortem distribution of AB-CHMINACA, 5-fluoro-AMB, and diphenidine in body fluids and solid tissues in a fatal poisoning case: usefulness of adipose tissue for detection of drugs in unchanged forms. Forensic Toxicology. 2015, 33(1), 45-53.

[9] Tamayo, F. G.; Turiel, E.; Martín- Esteban, A. Molecularly Imprinted Polymers for Solid-Phase Extraction and Solid-Phase Microextraction: Recent Developments and Future Trends. J. Chromatogr. A. 2017, 32, 1152.

[10] Whitcombe, M. J.; Kirsch, N.; Nicholls, I. A. Molecular Imprinting Science and Technology: a Survey of the Literature for the Years 2004-2011. J. Mol. Recogn. 2014, 27, 297-401.

[11] Sellergen, B.; Allender, C. J. Molecularly Imprinted Polymers: a Bridge to Advanced Drug Delivery. Adv. Drug Del. Rev. 2005, 57, 1733-1741.

[12] Wulff, G. Enzyme-like Catalysis by Molecularly Imprinted Polymers. Chem. Rev. 2002, 102, 1-28.

[13] Chianella, I.; Guerreiro, A.; Moczko, E.; Caygill, J. S.; Piletska, E. V.; De Vargas Sansalvador, I. M.; Whitcombe, M. J.; Piletsky, S. A. Direct Replacement of Antibodies with Molecularly Imprinted Polymer Nanoparticles in ELISA - Development of a Novel Assay for Vancomycin. Anal. Chem. 2013, 85, 8462-846.

[14] Yano, K.; Karube, I. Molecularly Imprinted Polymers for Biosensor Applications. Trends Anal. Chem. 1999, 18, 199-204.

[15] Tom, L. A.; Schneck, N. A.; Walter, C. Improving the Imprinting Effect by Optimizing Template:Monomer:Cross-linker Ratios in Molecularly Imprinted Polymer for Sulfasimethoxine, J. Chromatogr, B. 2012, 209. 61-64. 
[16] Haupt, K.; Mosbach, K. Molecularly Imprinted Polymers and Their Use in Biomimetic Sensors. Chem. Rev. 2000, 100, 2495-2504.

[17] Ye, L.; Haupt, K. Molecularly Imprinted Polymers as Antibody and Receptor Mimics for Assays, Sensors and Drug Discovery. Anal. Bioanal. Chem. 2004, 378, 1887-1897.

[18] Sarma, D.; Gawlitza, K.; Rurack, K. Polystyrene Core-Silica Shell Particles with Defined Nanoarchitectures as a Versatile Platform for Suspension Array Technology. Langmuir. 2016, 32, 3717-3727.

[19] King, H. A.; El-Sharif, H. F.; Matia-Gonzalez, A. M.; Iadevaia, V.; Fowotade, A.; Reddy, S. M.; Geber, A. P. Generation of Ribosome Imprinted Polymers for Sensitive Detection of Translational Responses. Sci. Rep. 2017, 7, 6542.

[20] Wackerlig, J.; Schirhagl, R. Applications of MIP nanoparticles and their advances towards industrial use - A Review. Anal Chem. 2016, 88, 250-261.

[21] Selvoni, G.; Marrazza, G. MIP-Based Sensors: Promising New Tools for Cancer Biomarker Determination. Sensors. 2017, 17, 718.

[22] Vandenryt, T.; van Grinsven, B.; Eersels, K.; Cornelis, p.; Kholwadia, S.; Cleij, T. J.; Thoelen, R.; De Ceuninck, W.; Peeters, M.; Wagner, P. Single-Shot Detection of Neurotransmitters in Whole-Blood Samples by Means of the Heat-Transfer Method in Combination with Synthetic Receptors. Sensors. 2017, 17, 2701.

[23] Zhao, W.; Zhang, R.; Sheng, X.; Cai, J.; Zhu, X.; Zhu, Y.; Wei, W.; Liu, X.; Luo, J. Molecularly Imprinted Polymeric Nanoparticles Decorated With Au NPs for Highly Sensitive and Selective Glucose Detection. Biosens. Bioelectron. 2018, 100, 497-503.

[24] Canfarotta, F.; Czulak, J.; Betlem, K.; Sachdeva, A.; Eersels, K.; van Grinsven, B.; Cleij, T. J.; Peeters, M. A Novel Thermal Detection Method Based on Molecularly Imprinted Nanoparticles as Recognition Elements. Nanoscale. 2018, 10, 2081-2089.

[25] Peeters, M. ; Troost , F. J.; Mingels, R. H. G.; Welsch, T.; van Grinsven, B.; Vranken, T.; Ingebrandt, S.; Thoelen, R.; Cleij, T. J.; Wagner, P. Impedimetric Detection of Histamine in Bowel Fluids Using Synthetic Receptors with pH-Optimized Binding Characteristics. Anal. Chem. 2013, 85, 1475-1483.

[26] Steen Redeker, E.; Eersels, K.; Akkermans, O.; Royakkers, J.; Dyson, D.; Nurekeyeva, K.; Ferrando, B.; Cornelis, P.; Peeters, M.; Wagner, P.; et al. Biomimetic Bacterial Identification Platform Based on Thermal Wave Transport Analysis (TWTA) through Surface-Imprinted Polymers. ACS Inf. Dis. 2017, 3, 388-397.

[27] Diliën, H.; Peeters, M.; Royakkers, J.; Harings, J.; Cornelis, P.; Wagner, P.; Steen Redeker, E.; Banks, C. E.; Eersels, K.; van Grinsven, B.; Cleij, T. J. Label-Free Detection of Small Organic Molecules by Molecularly Imprinted Polymer Functionalized Thermocouples: Toward In Vivo Applications. ACS Sens. 2017, 2, 583-589.

[28] Wackers, G.; Vandenryt, T.; Cornelis, P.; Kellens, E.; Thoelen, R.; De Ceuninck, W.; Losada Pérez, P.; van Grinsven, B.; Peeters, M.; Wagner, P. Array Formatting of the HeatTransfer Method (HTM) for the Detection of Small Organic Molecules by Molecularly Imprinted Polymers. Sensors. 2014, 14, 11016-11030.

[29] Ting, F.; Yang, W.; Wang, N.; Ni, X.; Wen, J.; Xu, W. Molecularly Imprinted Polymer Microspheres Derived from Pickering Emulsions Polymeriszation in Determination of di(2-ethylhexyl)phthalate in Bottled Water Samples. J. Appl. Poly. 2016, 133, 43484-43496.

[30] Fan, H.; Wang, J.; Meng, Q.; Tian, Y.; Xu, X.; Jin, Z. Photoirradiation Surface Molecularly Imprinted Polymers for the Separation of 6-o- $\alpha$-D-maltosyl- $\beta$-cyclodextrin, $J$ Sep Sci. 2017, 40, 4653-4660. 
[31] Haupt, K.; Dzgoev, A.; Mosbach, K. Assay System for the Herbicide 2,4Dichlorophenoxyacetic Acid Using a Molecularly Imprinted Polymer as an Artificial Recognition Element. Anal Chem. 1998, 70, 628-631.

[32] Eersels, K.; Diliën, H.; Lowdon, J. W.; Steen Redeker, E.; Rogosic, R.; Heidt, B.; Peeters, M.; Cornelis, P.; Lux, P.; Reutelingsperger, C. P.; Schurgers, L. J.; Cleij, T. J.; van Grinsven, B. A Novel Biomimetic Tool for Assessing Vitamin K Status Based on Molecularly Imprinted Polymers. Nutrients. 2018, 10, 751.

[33] Casadio, S.; Lowdon, J. W.; Betlem, K.; Tadashi, J.; Foster, C. W.; Cleij, T. J.; van Grinsven, B.; Sutcliffe, O.B.; Banks, C. E.; Peeters, M. Development of a Novel Flexible Polymer-Based Biosensor Platform for the Thermal Detection of Noradrenaline in Aqueous Solutions. Chem. Eng. J. 2017, 315, 459-468.

[34] Sulitzky, C.; Rückert, B.; Hall, A. J.; Lanza, F.; Unger, K.K.; Sellergen, B. Grafting of Molecularly Imprinted Polymer Films on Silica Supports Containing Surface-Bound Free Radical Initiators. Macromolecules. 2002, 35, 79-91.

[35] Anene, A.; Kalfat, R.; Chevalier, Y.; Hbaieb, S. Molecularly Imprinted Polymer-Based Materials as Thin Films on Silica Supports for Efficient Adsorption of Patulin. Colloids and surfaces A: physicochem. Eng. Aspects. 2016, 497, 293-303.

[36] Wei, X.; Li, X.; Husson, S. M. Surface Molecular Imprinting by Atom Transfer Radical Polymerization. Biomacromolecules. 2005, 6, 1113-1121.

[37] Zhang, H.; Jiang, J.; Zhang, H.; Zhang, Y.; Sun, P. Efficient Synthesis of Molecularly Imprinted Polymers with Enzyme Inhibition Potency by the Controlled Surface Imprinting Approach. ACS Macro Lett. 2013, 2, 566-570.

[38] Zhao, J.; Yu, H.; Chen, W.; Yang, R.; Zhu, J.; Liao, M.; Shi, D.; Zhang, G. Patterned Peeling 2D MoS off the Substrate. ACS Appl. Mater. Interfaces. 2016, 8, 15741-15749. 


\section{Supplementary Information}

\section{Supplementary Figure S1}

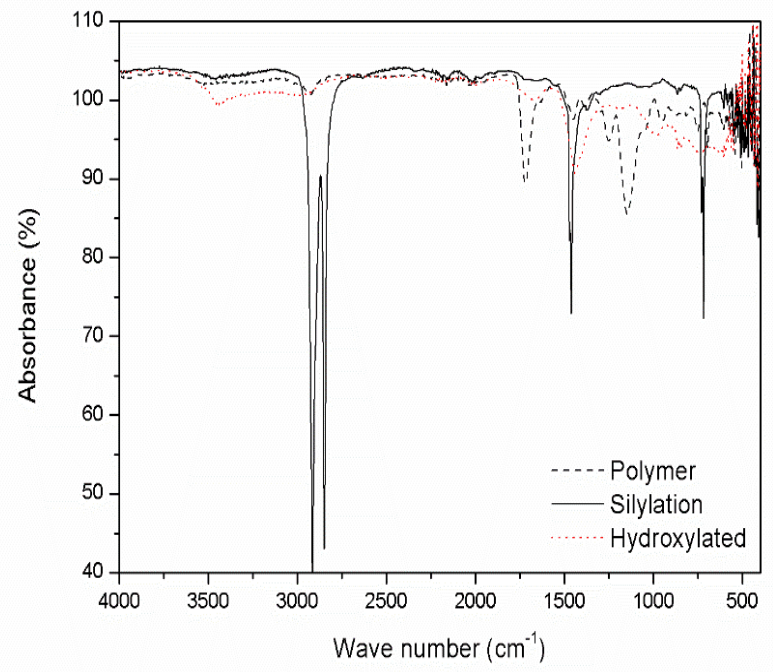

Figure. S1. FTIR spectrum of the different stages of modified aluminium, including hydroxylation, silylation and finally the grafting of the polymer

Table S1. Pre-polymerization mixture compositions tested for the grafting of imprinted polymers directly to aluminium surfaces.

\begin{tabular}{c|cccccc} 
MIP & $\begin{array}{c}\text { MAA } \\
(\mathbf{m L})\end{array}$ & $\begin{array}{c}\text { EGDMA } \\
(\mathbf{m L})\end{array}$ & $\begin{array}{c}\text { Toluene } \\
(\mathbf{m L})\end{array}$ & $\begin{array}{c}\text { Initiator } \\
(\mathbf{m g})\end{array}$ & $\begin{array}{c}\text { 2-MXP } \\
(\mathbf{m g})\end{array}$ & $\begin{array}{c}\text { Imprint } \\
\text { Effect }\end{array}$ \\
\hline 01 & 1 & 3 & 20.0 & 200 & 200 & 0.91 \\
02 & 1 & 4 & 20.0 & 200 & 200 & 3.49 \\
03 & 1 & 3 & 22.5 & 200 & 200 & 0.82 \\
04 & 1 & 3 & 25.0 & 200 & 200 & 1.03
\end{tabular}



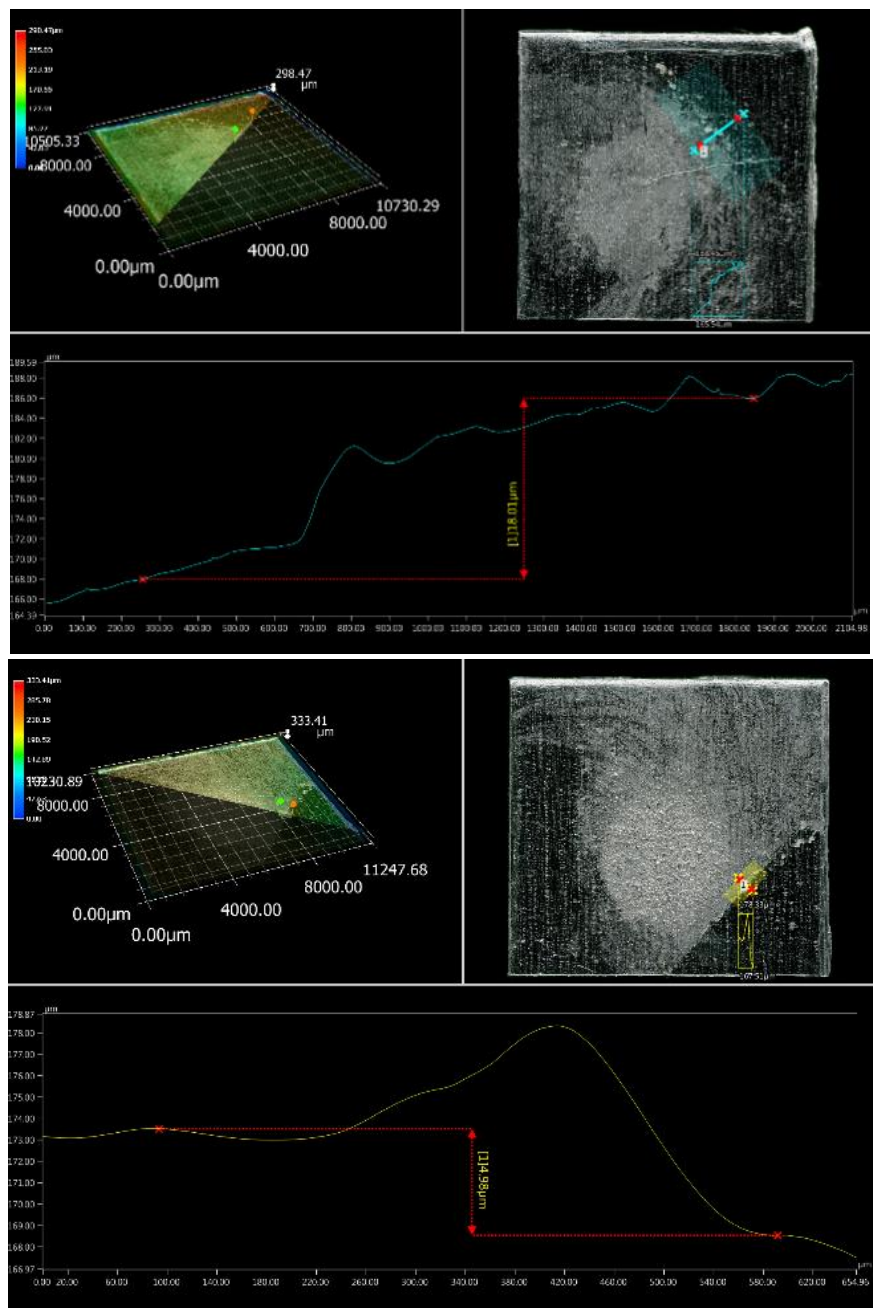

Figure S2. Profilometer measurements of MIP particles deposited into a spin coated adhesive PVC layer, with cross sectional analysis of layer thickness. (a) First sample analyzed using the deposition method and (b) another second sample prepared using the same deposition method for comparison. 

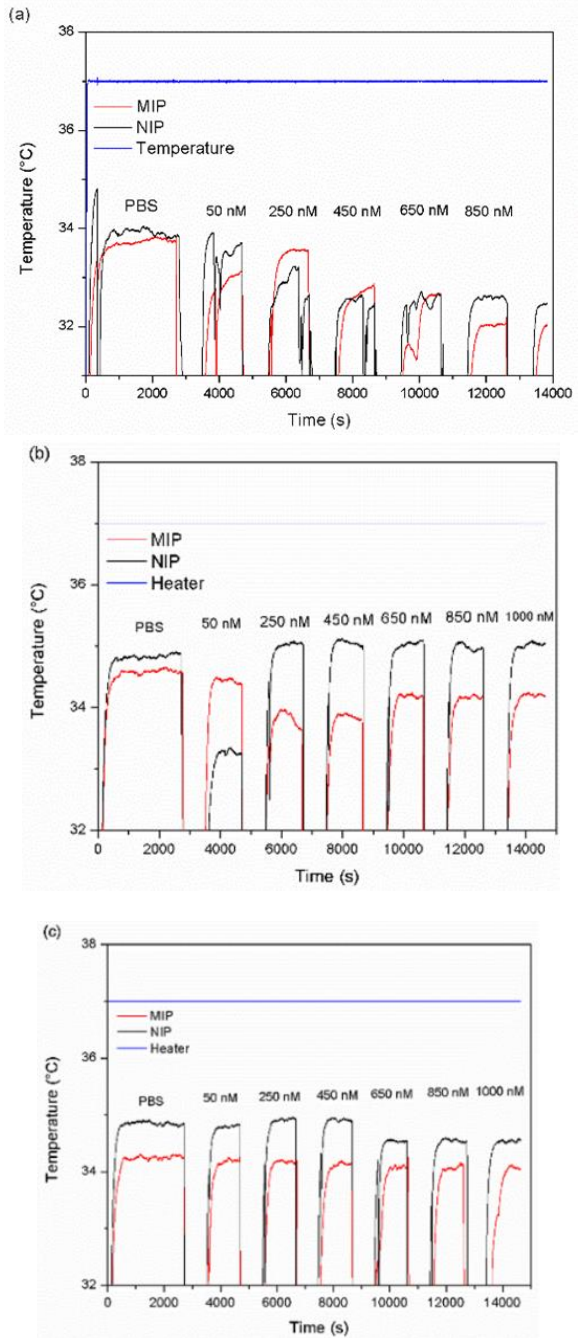

Figure S3. Temperature profiles determined using the HTM analysis of the surface grafted (a) MIP-01, (b) MIP-03, and (c) MIP-4 and the corresponding NIPs using varying concentrations $(50-1000 \mathrm{nM})$ of 2-MXP. 
<smiles>COc1ccccc1C(Cc1ccccc1)N1CCCCC1</smiles>

(a)<smiles>CC(=O)Nc1ccc(O)cc1</smiles>

(b)<smiles>Cn1c(=O)c2c(ncn2C)n(C)c1=O</smiles>

(c)

(d)<smiles>c1ccc(CC(c2ccccc2)N2CCCCC2)cc1</smiles>

(e)

Figure S4. Structures of competitive analytes (a) 2-MXP, (b) Paracetamol, (c) Caffeine, (d) Sucrose, and (e) Diphenidine 


\section{Chapter 4}




\section{Substrate displacement colorimetry (SDC) preface:}

Based on the previous publication/chapter a new approach was taken when attempting to design a mip-based sensory platform. Previously, a change in thermal resistance was used as an indicator for the presence of a target substrate, and though effective, the methodology still requires a readout platform to successfully translate a binding event into a tangible signal. An approach based on a transducing principal that omits the need for any instrumentation entirely was therefore investigated, transforming a binding interaction at the surface of the MIP into an event that can be perceived with the naked eye.

The developed method is based on a dye displacement assay, where a MIP is produced for a desired target compound and preloaded with a dye. Incubation with the target compound leads to the release of said dye into the surrounding medium, and therefore the presence of the target molecule is visually confirmed. Variations of this technology have been demonstrated before, but no variation investigated the preloading of the dye molecule before reintroduction of the template.

This approach enables a MIP to be utilized in a manner where little modification to the base MIP composition is required, and removes the need for a transducer. This therefore makes the technology particularly valuable for in-the-field-testing, where equipment is limited. It can be imagined that this technology could have a large impact in the field of rapid drug sensing, being able to confirm the presence of a compound without expensive or time consuming analysis. 


\section{Research paper: Substrate Displacement Colorimetry for the Detection of Diarylethylamines}

Joseph W. Lowdon ${ }^{1}$, Kasper Eersels ${ }^{1}$, Renato Rogosic ${ }^{1}$, Benjamin Heidt $^{1}$, Hanne Diliën ${ }^{1}$, Erik Steen Redeker ${ }^{1}$, Marloes Peeters ${ }^{2}$, Bart van Grinsven ${ }^{1}$, Thomas J. Cleij ${ }^{1}$

${ }^{1}$ Maastricht Science Programme, Maastricht University, PO Box 616, 6200 MD, Maastricht, the Netherlands

${ }^{2}$ Manchester Metropolitan University, Faculty of Science and Engineering, School of Science and the Environment, Division of Chemistry and Environmental Science, Chester Street, Manchester M1 5GD, United Kingdom. 


\begin{abstract}
In this work, a novel detection assay for the new psychoactive substance (NPS) 2-methoxiphenidine (2-MXP) and other diarylethylamines is introduced. The assay is based on the competitive displacement of dye molecules from molecularly imprinted polymers (MIPs) by the target molecule. The assay was fully characterized by studying the affinity of the MIP for six common dyes, expressed as the binding factor (BF). The results of this study indicate that the mathematical relationship between the $\mathrm{BF}$ of a dye and the imprinting factor (IF) for the target could be used for the prediction of the efficacy of the displacement assay. Dye-loaded MIP particles where incubated with the target, two adulterants and two legal pharmacological compounds. The target has a higher affinity for the MIP than the dye and displaces it out of the nanocavities of the receptor leading to a colour change in the filtrate that can be observed with the naked eye. Incubation of the MIP particles with the adulterants and legal medicines did not result in any observable change in absorbance. The robust, fast and low-cost nature of the assay, combined with its tailorable selectivity and generic nature, illustrate its potential as a prescreening tool for the identification of narcotic substances in unidentified powders.
\end{abstract}

Keywords: Molecularly imprinted polymer, displacement assay, colorimetry, new psychoactive substance identification 


\section{Introduction}

Molecularly imprinted polymers (MIPs) have emerged as synthetic receptors in a wide range of applications over the past few decades [1,2]. Drawing parallels with enzymes, these MIPs contain nanocavities that serve as selective binding pockets for a specific substrate [3]. The origin of MIP selectivity lies in the synthesis process, where a polymer is crosslinked in the presence of a template molecule [4]. The affinity of the MIP towards the target can be tailored by fine-tuning the constituent components and ratios. In this way, MIPs can display binding affinities for their target that are similar to biological receptors but are able to withstand harsh physical and chemical conditions in contrast to their natural counterparts [5-7]. Along with their generic, low-cost synthesis process, this makes MIPs interesting for incorporation into biomimetic sensing devices for the detection of a wide variety of compounds in biological samples [8-12].

MIPs have advanced much over years, evolving from bulk polymerized monoliths to more elegant nanoparticles that form the basis of many biosensing devices [13-16]. The replacement of biological receptors by MIPs in alternatives to classical competitive assays in biomedicine was introduced by Vlatakis et al. with the advent of so-called molecular imprinting sorbent assays (MIAs) [17]. Traditionally, these assays combined bulk polymerized MIPs and radio-labelled analytes in a competitive binding assay, studied by spectroscopic methods [18]. Other assays were soon introduced, based on the competitive binding of the target and fluorescent-labelled [19] and enzymelinked substrates [20], as radio-labelling is expensive and poses a health risk. Alternatively, displacement assays were developed that made use of MIP particles pre-loaded with fluorescently labelled target molecules [21, 22]. Incubation of these pre-loaded particles with a solution containing the target resulted in displacement of the labelled-substrates which could be assessed by fluorescent microscopy. The high selectivity and sensitivity of these assays makes them extremely suitable for the detection of trace amounts of analyte but many applications do not require these extreme degrees of sensitivity and selectivity. In this light, several displacement assays were developed that were based on the displacement of a dye that could be observed by absorption spectroscopy, omitting the need for an expensive and labour-intensive procedure such as fluorescent labelling [23-25].

In this study, a novel drug detection assay, coined substrate displacement colorimetry (SDC), is introduced. The assay is able to detect several narcotic compounds based on the colorimetric detection of displaced dye molecules. MIPs were synthesized for the new psychoactive substance (NPS), 
methoxphenidine (2-MXP). The classical bulk imprinting approach was chosen due to its easy and scalable synthesis procedure. The synthesis protocol was optimized and binding of six commonly used dyes to the MIP was analyzed to determine the binding factor $(\mathrm{BF})$ for each dye. This BF was compared to the MIP imprinting factor (IF) to establish a straightforward prediction model for assessing dye-target combinations. Next, MIPs were loaded with malachite green and washed thoroughly to ensure complete removal of any unbound or weakly bound dye molecules. When exposing these dye-loaded MIPs to a sample containing their target, the dye will be displaced due to the higher affinity the MIP has towards the target analyte, releasing the dye into the surrounding medium (Figure. 1).

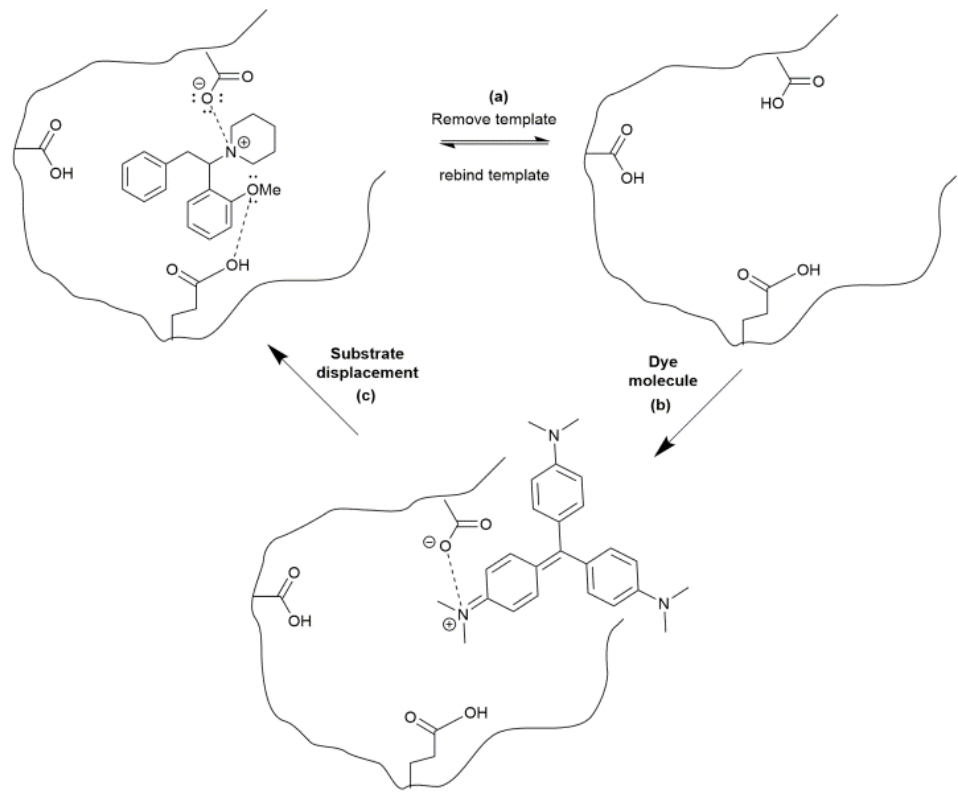

Figure. 1. Substrate displacement colorimetry principle. Extracted MIPs (2-MXP) are loaded with a structural similar dye (crystal violet). When the MIP is incubated with its original template, the dye is displaced from the binding pockets and released into the surrounding medium.

Most low-cost field colour tests suffer from false positives when analysing samples containing adulterants or legal pharmacological compounds and the use of MIPs as synthetic receptors in this assay could overcome this problem to a large extent [26]. The current assay does not display any cross-selectivity 
between 2-MXP, two common adulterants and two legal pharmacological compounds. At the same time, the assay is able to detect other diarylethylamines in addition to 2-MXP. In this way, the experiments summarized in this paper illustrate a proof-of-concept for a low cost drug test that allows the end-user to visually determine the presence of narcotics in an unidentified powder and selectively identify e.g. an NPS in a complex mixture, a task that proves hard for routinely used, low-cost drug tests. In addition, a simple absorbance measurement on a spectrophotometer suffices to quantify even tiny amounts of 2-MXP that do not yield a colour reaction that is visible with the bare eye.

The use of SDC for the rapid and low-cost identification of NPS in unknown powders can be considered highly relevant, as the production and distribution of these compounds has expanded tremendously in recent years. NPS are typically based on legitimate pharmaceutical compounds and illegal psychoactive pharmacophores and their popularity and presence rises as it allows both users and narcotic producers to avoid legal restrictions [27]. Although these compounds are sometimes referred to as "legal highs" they are every inch as dangerous and addictive as their natural counterparts, leading to over-dose related deaths, physical and mental addiction and long-term damage to the central nervous system leading to a wide variety of conditions including psychosis and suicidal ideation [28, 29]. In response to authorities banning defined substances, the specific compounds on the market change rapidly. This makes it hard to study their effects, reinforce proper legislation and inform potential users of the dangerous effect of abusing these substances. In addition, rapid and specific detection of these compounds is vital in terms of adequate treatment and timely intervention. Therefore, a low-cost drug test that allows for selective detection of these compounds and their derivatives would be useful as most current drug test tend to yield false negatives when analysing NPS [26].

The results described in this paper show the potential use of this novel, lowcost assay in selectively detecting narcotic compounds in unidentified powders. The selectivity of the MIP makes the test superior over other commercial routine screening tools while the ability to still recognize similar compounds of the same drug class, will avoid drug producers avoiding legislation by continuously changing the synthesis protocol. The possibility of quantifying the response using simple spectrophotometry, illustrates its potential for more in-depth analysis of complex samples containing trace amounts of illicit substances as an alternative to expensive state-of-the-art techniques based on mass spectrometry that are usually confined to a laboratory setting. Although the concept has only been demonstrated for one 
class of NPS, it can be expected that the technology can be extended to other targets in a fairly fast manner given the constant scientific progress within the field of molecular imprinting. Additionally, the MIP-based displacement approach could be extended towards other diagnostic applications in the future increasing its potential impact on the field of biomimetic sensing dramatically. 


\section{Materials and methods}

\section{Chemicals and reagents}

Prior to polymerization, stabilizers were removed from the functional and crosslinking monomers by passing the solutions over a column packed with alumina. All chemicals and solvents were obtained from Sigma Aldrich. All solutions were prepared with deionized water of resistivity of $18.2 \mathrm{M} \Omega \mathrm{cm}$ or with phosphate buffered saline (PBS) solutions.

\section{Synthesis of molecularly imprinted polymers}

The synthesis protocol was optimized by varying the ratios of monomers, target and crosslinker (Supplementary Information Table S1). The best results were obtained by dissolving a mixture of the functional monomer methacrylic acid (MAA $1.02 \mathrm{mmol}$ ), crosslinker molecule ethylene glycol dimethacrylate (EGDMA $1.7 \mathrm{mmol}$ ), and initiator azobisisobutyronitril (AIBN $0.30 \mathrm{mmol}$ ) in dimethyl sulfoxide (DMSO) together with the template molecule $(0.17 \mathrm{mmol})$. The mixture was purged with $\mathrm{N}_{2}$ before the initiation of the polymerization. Polymerization was performed by heating the mixture up to $65^{\circ} \mathrm{C}$ for $12 \mathrm{~h}$, allowing for full completion of the reaction. MIPs and NIPs were milled seven times using a Fritsch Planetary Micro Mill Pulverisette 7 premium line (700 $\mathrm{rpm}, 5$ minutes, $10 \mathrm{~mm}$ balls). After milling, the particles were sieved at $1.0 \mathrm{~mm}$ amplitude using a Fritsch Analysette 3 for 4 hours or until sufficient amount of polymer was on the collection plate to achieve microparticles with sizes smaller than $100 \mu \mathrm{m}$. Finally, the template molecule was removed from the MIP powders by continuous Soxhlet extraction with a 1:10 mixture of acetic acid and methanol for $12 \mathrm{~h}$, followed by further extraction with pure methanol for a further $12 \mathrm{~h}$. Extraction was verified using FTIR (see Supplementary Information Fig. S1). The MIP powder was then dried at $60^{\circ} \mathrm{C}$ overnight, yielding the extracted dried MIP powder. The NIP was prepared in the same manner, without the presence of the template molecule. MIP morphology was studied using scanning electron microscopy (see Supplementary Information Fig. S2).

\section{Batch rebinding experiments}

Optical batch rebinding experiments were evaluated with a Shimadzu UV3600 spectrophotometer. A $1 \mathrm{mM}$ stock solution consisting of PBS and target substrate was used to prepare an array of solutions varying between $0-0.7$ $\mathrm{mM}$. To $5 \mathrm{ml}$ of each solution $20 \mathrm{mg}$ of MIP/NIP powder was added, and the resulting suspensions placed on an orbital shaker $(125 \mathrm{rpm})$ for $1 \mathrm{~h}$ at room 
temperature. After filtration, the free concentration of substrate in the filtrate was determined by UV-vis spectroscopy and HPLC analysis, allowing binding isotherms to be constructed. As a measure of specificity and in order to compare the different MIP compositions, the imprint factor (IF) was determined at $C_{\mathrm{f}}=0.05 \mathrm{mM}$. To determine whether the MIP could selectively extract the target molecule from mixtures, selectivity tests were performed with other substrates. This was done for all MIPs under study.

\section{Preparation of dye-loaded MIP}

To $20 \mathrm{ml}$ of aqueous dye molecule $(1 \mathrm{mM}), 500 \mathrm{mg}$ of previously prepared MIP powder was added, and proceeded to be left on an orbital shaker $(250 \mathrm{rpm})$ for $2 \mathrm{~h}$. The resulting suspension was then filtered and the remaining filtered solid washed with distilled water until the filtrate ran colourless. The coloured MIP powder was then placed in an oven overnight at $90^{\circ} \mathrm{C}$ to yield a dry powder. 2-MXP MIPs were loaded with crystal violet, malachite green, methyl orange, basic blue, phenol red, or pararosailine respectively to study the SDC principle and to examine the effect of combining different dyes with different MIPs.

\section{Kinetic analysis of Substrate Displacement Colorimetry (SDC)}

The SDC assay was initiated by adding $20 \mathrm{mg}$ of dye-loaded MIP powder to 5 $\mathrm{mL}$ of aqueous 2-MXP (1 mM). After varying fixed time intervals $(1,2,3,4,5$, 10, 15 and 20 minutes) of incubation on an orbital shaker, the solutions were filtered and the colour of the filtrate observed. If there was a significant amount of binding between the 2-MXP and the dye-loaded MIP, the dye would be displaced and released into solution, resulting in a colour change after filtration. Filtrates were examined both visually and optically using a Shimadzu UV-3600 spectrophotometer, to build a relationship between the dye displacement and the incubation time.

\section{Substrate displacement Colorimetry (SDC) selectivity analysis}

The SDC assay was initiated by adding $20 \mathrm{mg}$ of dye-loaded MIP powder to 5 $\mathrm{mL}$ of aqueous analyte $(1 \mathrm{mM})$. After a one minute of incubation on a shaker, the solutions were filtered and the colour of the filtrate observed. This was repeated for all MIPs under study and used to analyze samples containing caffeine, paracetamol, aspirin, and sucrose. If there was a significant amount of binding between the substrate and the dye-loaded MIP, the dye would be displaced and released into solution, resulting in a colour change after filtration. Filtrates were examined both visually and optically using a Shimadzu UV-3600 spectrophotometer. As NPS have ever evolving minor 
structural changes, the dye-loaded MIP was also exposed to other diarylethylamines, assessing if the assay would be able to cope with continuously changing compositions of NPS.

\section{SDC Quantitative analysis of loaded 2-MXP MIP}

To examine the potential for quantifying drugs aqueous solutions containing varying concentrations $(0.01-1 \mathrm{mM})$ of $2-\mathrm{MXP}$ and caffeine were incubated with $20 \mathrm{mg}$ of malachite green-loaded 2-MXP MIPs and shook on an orbital shaker (125 rpm) for 5 minutes. The solutions were then filtered and the absorbance of the malachite green displaced into solution was determined by UV-vis spectroscopy. All measurements were performed in triplet and a mean absorbance and standard error was calculated for each concentration. The data were fit using Origin Pro 8. 


\section{Results and discussion}

\section{Batch rebinding analysis}

Table 1. Imprint factors and binding capacities of the MIPs synthesized templated with 2MXP

\begin{tabular}{c|cc} 
MIP & IF & $\begin{array}{c}\text { Binding capacity } \\
\left(\mu \mathbf{m o l ~ g} \mathbf{~ g}^{-1}\right)\end{array}$ \\
MIP-21 & 1.02 & 141 \\
MIP-22 & 1.32 & 146 \\
MIP-23 & 1.01 & 156 \\
MIP-24 & 1.83 & 159 \\
MIP-25 & 1.07 & 149
\end{tabular}

The specificity and binding capacity of MIPs optimized for the detection of 2MXP were analyzed by means of UV-vis spectroscopy. The results obtained in batch rebinding experiments with both functionalized MIPs and their nonimprinted reference (non-imprinted polymers or NIPs) were used to construct binding isotherms by plotting the amount of target bound per gram of polymer $(\mathrm{Sb})$ in function of the free concentration $\left(\mathrm{C}_{\mathrm{f}}\right)$ of target remaining in solution. In order to determine the imprinting factor (IF), the data, shown in Figure. 2, were fit using an allometric (2-parameter) fit that reflects the heterogeneous nature of the binding sites within the MIP particles. The IF was calculated as the relative amount of binding by the MIP in comparison to the NIP at $0.05 \mathrm{mM}$ using the allometric fit, demonstrating various IF values and binding capacities (Table 1). Of the MIP/NIPs tested, MIP-24 demonstrated the highest IF of 1.83 and a binding capacity of $159 \mu \mathrm{mol} \mathrm{g}{ }^{-1}$. 


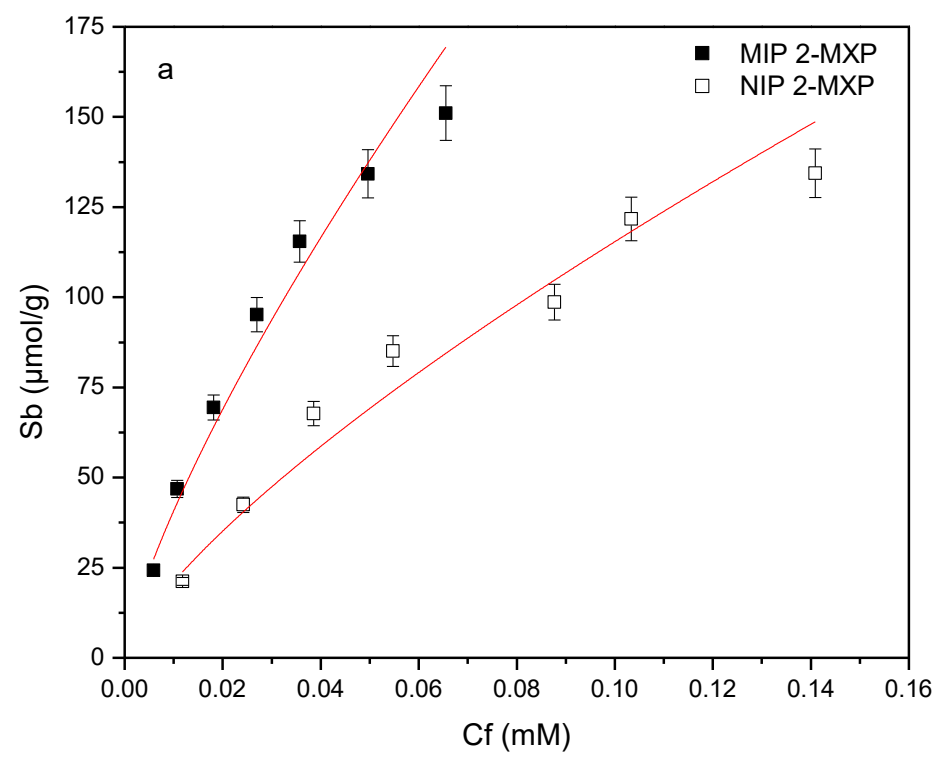

Fig. 2. Batch rebinding analysis on a UV-vis spectrometer: both a MIP imprinted for 2MXP and a NIP reference were exposed to increasing concentrations of 2-MXP in water. Error bars are representations of the standard error over three measurements. The binding isotherms show that the MIP is able to bind the target in a specific manner and an imprinting factor of 1.83 can be observed at $0.05 \mathrm{mM}$.

\section{Batch rebinding analysis: optimizing dye-MIP coupling}

Rational design of the SDC assay was simulated by a similar batch rebinding experiment as the one described in the previous chapter. 2-MXP MIPs were incubated with six common, readily available dyes with structures that favour the formation of hydrogen bonds (see Supplementary Information Fig. S3). The resulting binding isotherms show that the MIP has the highest affinity for malachite green. The highest degree of specific binding, which was expressed as the binding factor $(\mathrm{BF})$, was also observed for malachite green. BF was calculated in an analogous way to the imprinting factor (IF) for the target but distinguishes from this classical term as the dyes were not imprinted. The resulting BFs and the extinction coefficients for each of the dyes were summarized in Table 2. Malachite green was chosen as a dye due to its superior binding factor and resulting positive effect on the selectivity of the assay. In addition, the use of malachite green as a loading dye for 2-MXP MIPs was 
evaluated due to its high extinction coefficient, which should increase the assay's sensitivity.

Table 2. Binding factors and extinction coefficients for methyl orange, crystal violet, phenol red, basic blue, pararosaniline and malachite green upon incubation with MIP-24 particles.

\begin{tabular}{|c|c|c|}
\hline Dye & Binding Factor (BF) & $\begin{array}{l}\text { Extinction } \\
\text { coefficient }\end{array}$ \\
\hline $\begin{array}{c}\text { Malachite green }(\lambda=616 \\
\mathrm{nm})\end{array}$ & 1.19 & 148,000 \\
\hline $\begin{array}{c}\text { Crystal Violet }(\lambda=590 \\
\mathrm{nm})\end{array}$ & 1.05 & 87,000 \\
\hline $\begin{array}{l}\text { Methyl Orange }(\lambda=464 \\
\mathrm{nm})\end{array}$ & 0.39 & 20,500 \\
\hline Basic Blue $(\lambda=616 \mathrm{~nm})$ & 0.81 & 62,000 \\
\hline Pararosaline $(\lambda=616 \mathrm{~nm})$ & 0.99 & 80,000 \\
\hline Phenol Red $(\lambda=432 \mathrm{~nm})$ & 0.98 & 55,000 \\
\hline
\end{tabular}


Table 3. Absorbance of the filtrates collected when MIP-24 loaded with either malachite green, phenol red, crystal violet, methyl orange, pararosaniline, or basic blue, was exposed to 2-MXP (1 mM) for 1 minute.

\begin{tabular}{c|cc} 
Pre-loaded Dye & Absorbance & Colour of filtrate \\
\hline $\begin{array}{c}\text { Malachite green }(\lambda=616 \\
\text { nm })\end{array}$ & 0.0750 & Blue \\
$\begin{array}{c}\text { Crystal Violet }(\lambda=590 \\
\mathrm{nm})\end{array}$ & 0.0182 & Colourless \\
$\begin{array}{c}\text { Methyl Orange }(\lambda=464 \\
\text { nm })\end{array}$ & 0.0000 & Colourless \\
Basic Blue $(\lambda=616 \mathrm{~nm})$ & 0.0000 & Colourless \\
Pararosaline $(\lambda=616 \mathrm{~nm})$ & 0.0000 & Colourless \\
Phenol Red $(\lambda=432 \mathrm{~nm})$ & 0.0000 & Colourless
\end{tabular}

To demonstrate that malachite green was indeed the best available dye to be used in this assay, MIP particles were loaded with crystal violet, methyl orange, basic blue, phenol red, pararosaniline and malachite green and exposed to 2-MXP. The colours of the resulting filtrates were observed with the naked eye and the absorption was measured using the spectrophotometer (Table 3). Malachite green has the highest degree of displacement, having a visually observable coloured filtrate (Supplementary Information Fig. S4). The other dyes did not produce a noticeable difference in colour of filtrate, although a small amount of crystal violet could be detected with the spectrophotometer. These findings are in line with their low BFs, indicating that they will mostly occupy non-specific binding areas of the polymer and are easily removed during the washing step. The displacement is presumed to occur at the nanocavities where the 2-MXP has a higher affinity than the dye, displacing it out of the cavities into the surrounding solution. As the coloured MIP particles will be removed by means of a syringe filter the filtrate will only be coloured when the dye was displaced. 


\section{Batch rebinding analysis: optimizing dye displacement}

Table 4. Imprint factors and binding factors when MIPs have been exposed to the target 2-MXP, and the dye malachite green, $\Delta \boldsymbol{B} \boldsymbol{F}$ indicates the difference in binding between the target molecule and the dye. Absorbance readings displayed relate to the absorbance of the malachite green after the dye loaded MIP has been incubated with 2-MXP $(1 \mathrm{mM})$, and the colour of the filtrate observed.

\begin{tabular}{c|ccccc} 
MIP & $\begin{array}{c}\text { IF (2- } \\
\text { MXP) }\end{array}$ & $\begin{array}{c}\text { BF } \\
\text { (Malachite } \\
\text { green) }\end{array}$ & $\Delta \boldsymbol{B F}$ & $\begin{array}{c}\text { Absorbance } \\
(\boldsymbol{\lambda}=\mathbf{6 1 6} \mathbf{n m})\end{array}$ & $\begin{array}{c}\text { Colour of } \\
\text { filtrate }\end{array}$ \\
\hline MIP-21 & 1.02 & 1.42 & -0.40 & 0.0000 & Colourless \\
MIP-22 & 1.32 & 2.33 & -1.01 & 0.0000 & Colourless \\
MIP-23 & 1.01 & 1.22 & -0.21 & 0.0000 & Colourless \\
MIP-24 & 1.83 & 1.19 & 0.64 & 0.0750 & Blue \\
MIP-25 & 1.07 & 1.04 & 0.03 & 0.0121 & colourless
\end{tabular}

The observed binding factor of the dye is only one parameter that must be considered when performing substrate displacement. If the MIP has a greater affinity for the dye than for the target, no displacement will occur, and therefore IF also plays a role in the efficacy of the displacement process. To demonstrate this concept, previously synthesized MIPs for 2-MXP were incubated with malachite green in a similar manner to the previous rebinding experiments. The binding isotherms generated from this allowed for the calculation of the binding factors of the malachite green towards each MIP (Table 4). The difference between the IF and BF is designated $\triangle \mathrm{BF}$ and gives an indication to the differences in specific binding of the MIP towards malachite green and 2-MXP. As $\triangle \mathrm{BF}$ becomes more positive, the likelihood of dye displacement increases. This is due to the target molecule having a higher affinity towards the MIP than the currently bound dye molecule, as the imprinting effect favours the target molecule. From the experimental data a curve was constructed, depicting this mathematical relationship between $\mathrm{BF}$ and IF (Fig. 3). The dashed line in the graph represents the point where BF and IF are equal. Any MIPs that have data points below this line will not exhibit displacement properties as the binding of the dye is greater than that of 2-MXP. Confirmation of this theory was conducted by preloading each of the MIPs 
with malachite green, and then incubating the loaded MIPs with 2-MXP (1 $\mathrm{mM}$ ). MIP-24, the MIP with the highest $\triangle \mathrm{BF}$, shows clear displacement of the dye that can be visually observed (Supplementary Information Fig. S5) while MIP 25 displaces less dye making visual observation more difficult. These results were confirmed with UV-vis spectroscopy (Table 4).

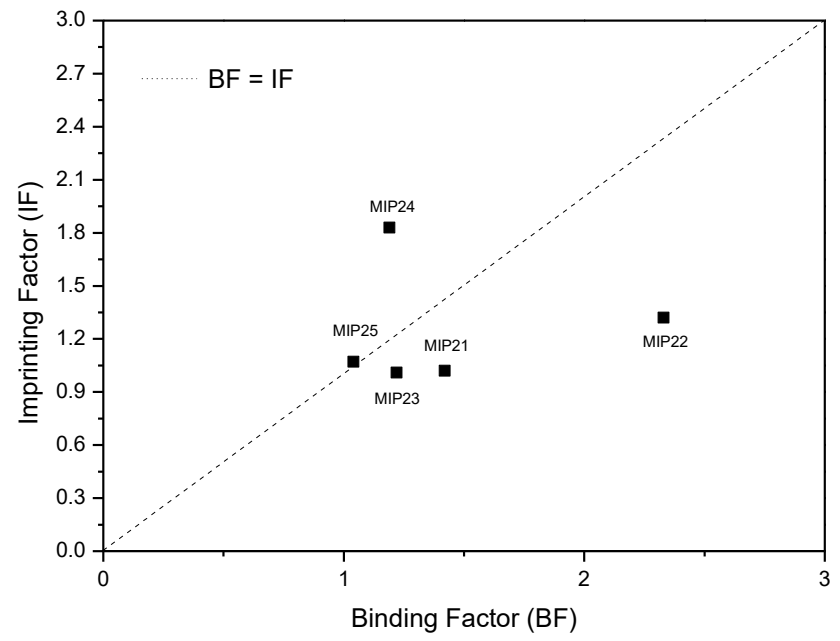

Fig 3. Imprint factor - Binding factor graph for varying compositions of MIPs that are imprinted for 2-MXP. Imprint factor is in relation to 2-MXP, and binding factor is in relation to malachite green.

\section{SDC: Incubation time dependency}

To get an insight into the kinetic behaviour of the dye displacement process, the effect of varying the incubation time on dye displacement was released. The malachite green-loaded MIPs were incubated with 2-MXP $(1 \mathrm{mM})$ for different amounts of time, varying from 1 minute to 20 minutes. The UV absorbance of the dye displaced was measured for each time period, to determine the optimal operating time (Fig 4). A clear coloured filtrate is already observed within a minute after incubation and a time-dependent, linear increase in colour intensity could be observed during the first 10 minutes. The UV-vis data indicate that the curve levels after 10 minutes eventually plateauing at 20 minutes, indicating that no additional dye is being released from the MIP. These results indicate that detecting trace amounts of narcotics in unknown powders in a quantitative manner will probably require 
longer time intervals but, depending on the concentration of drugs in the sample, a fast test can also be performed.

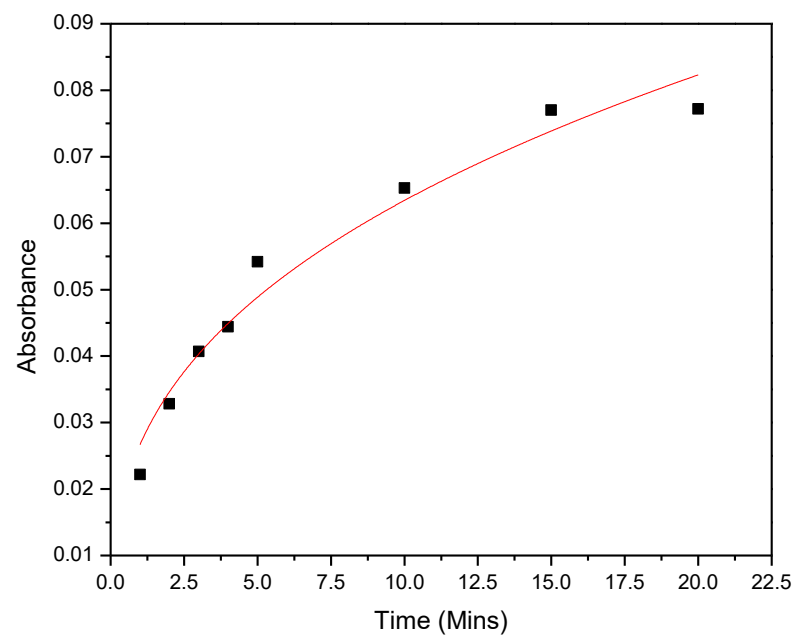

Fig 4. Time dependent elution isotherm for when malachite green loaded MIP-24 is incubated with 2-MXP $(1 \mathrm{mM})$ for varying time intervals. The UV absorbance of the malachite green was measured using a UV-spectrometer, to determine the amount of dye displaced.

\section{Selectivity of the SDC}

The selectivity of the MIP towards other pharmaceutical compounds (paracetamol and aspirin) and common adulterants (caffeine and sucrose) was assessed to come to a primary evaluation of the MIP's potential to discriminate an NPS from other morphologically similar white powders. The batch rebinding analysis, (shown in Supplementary Information Fig. S6) indicates that the MIP is very selective towards its target. The binding capacity of the MIP for all these compounds (structures summarized in Supplementary Information Fig. S7) is lower in comparison to the template and IFs do not significantly deviate from 1 .The assay shows a similar trend; incubating MIPs, pre-loaded with malachite green, with any of the analogue powders, did not result in any visible colour change in the filtrate (See Supplementary Fig. S8). Further analysis of the filtrates by means of UV-absorbance readings $(\lambda=616$ $\mathrm{nm}$, Malachite green's most intense absorbance peak, Table 5) confirmed that the dye was not present in the filtrate. The presence of $2-\mathrm{MXP}$, is the only 
compound tested that elicited a response from the dye loaded MIP, showing the selectivity of the MIP against other possible chemical architectures.

Table 5. Compounds of different chemical classes (a) and compounds of the same class as 2-MXP (b) were incubated with the malachite green loaded MIP. The filtrates were then collected UV-absorbance measured $(\lambda=616 \mathrm{~nm})$ and colour of filtrate observed

\begin{tabular}{c|cc} 
(a) Different & Absorbance & Colour \\
substrate class & 0 & Colourless \\
\hline Blank & 0 & Colourless \\
Caffeine & 0.0750 & Blue \\
2-MXP & 0 & Colourless \\
Paracetamol & 0 & Colourless \\
Sucrose & 0 & Colourless \\
Aspirin & & \\
(b) Same substrate & & Blue \\
class & 0.0750 & Blue \\
2-MXP & 0.0217 & Blue \\
3-MXP & 0.0261 & Blue \\
4-MXP & 0.0193 & Blue \\
Diphenidine & 0.0131 & Blue \\
2-MEPE & 0.0103 & Blue \\
3-MEPE & 0.0163 & Blue \\
4-MEPE & 0.0998 &
\end{tabular}

However, 2-MXP falls in the class of compounds known as diarylethylamines that are known to induce psychoactive effects when consumed. Drug producers tend to slightly modify the structure of these compounds to avoid legislation. Therefore, known psychoactive diarylethylamines are shown to be structural analogues or region-isomers of 2-MXP (structures summarized in Supplementary Information Fig. S9), giving them a high affinity towards the MIP. This apparent limited selectivity of the MIP system could be of great analytical use because it allows for the detection of other diarylethylamines without the need for synthesizing a MIP for each new NPS of this class that reaches the market. MIP particles, pre-loaded with malachite green, were incubated with other aqueous solutions of diarylethylamines, leading to displacement of the dye and visibly coloured filtrates (Supplementary 
Information Fig. S10), UV-absorbance readings were taken to confirm displacement (Table 5). This illustrates that the assay can be tailored towards a broader array of analytes sharing a similar structure.

\section{SDC: quantitative analysis}

To verify whether it is possible to quantify the colorimetric response, malachite green-loaded and crystal violet-loaded MIP powders were incubated with increasing amounts of 2-MXP and caffeine (0.01 - $1 \mathrm{mM}$ for both compounds) and analyzed using a UV-vis spectrometer to determine the amount of target present in the analyte. The data shown in Figure 5a shows that the MIP releases dye in a concentration-dependent manner when exposed to increasing amounts of 2-MXP. Exposing the dye-loaded MIP to caffeine does not result in any measurable displacement of the dye over the entire concentration range. In order to determine the limit-of-detection $(\mathrm{LoD})$ the lower concentration range was plotted and analyzed separately in Figure $5 \mathrm{~b}$. The data were fit using a linear regression line $\left(R^{2}=0.95653\right)$ and the $3 \sigma$ method was used to establish a detection limit of $50 \mu \mathrm{M}$. This indicates that the technology can be used to detect micrograms of unknown powders dissolved in milliliter amounts of water.
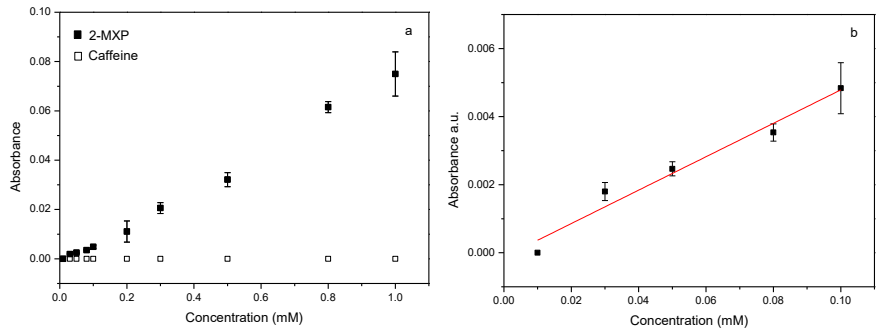

Fig. 5. Quantitative 2-MXP assay: to determine the limit-of-detection (LoD) for the SDC assay using 2-MXP MIPs loaded with malachite green. The plot over a wide concentration range indicates that the response for 2-MXP can be quantified while exposing the MIP to an increasing concentration of caffeine does not result in any measurable displacement of the dye (a). Zooming in on the lower concentration range shows a linear increase (R2 = 0.95653) and an LoD of $50 \mu \mathrm{M}$ was calculated using the $3 \sigma$ method (b). 


\section{Conclusion}

The results in this paper nicely illustrate the underlying principles of a substrate displacement strategy that relies upon MIPs for their selectivity. Structural similarities and similar spatial orientation of functionalities between the dye and target molecule (2-MXP) were exploited to create a selective detection assay. Out of the dyes tested, malachite green was found to show the strongest specific affinity towards the MIP and the highest binding capacity when bound. The intuitive relationship between the binding factor of a dye and the imprinting factor of the target can be mathematically assessed to predict correct functioning of the assay. The high extinction coefficient of malachite green also enabled for the detection low amounts of drugs. The kinetic study on the MIP-based assay revealed that application in drug detection assays depends on the specific application at hand and a trade-off between fast, reaction times and sensitivity needs to be taken into account. Although the assay has demonstrated to be able to selectively discriminate between NPS and compounds that can yield false negatives, it is not too selective to enable drug producers and users to evade the test by slightly modifying their synthesis process as the test is responsive to other diarylethylamines too.

The quantitative nature of the assay has the additional benefit that although trace amounts of drug molecules might not result in a color reaction that can be seen with the bare eye, the spectrophotometer is able to pick up amounts as low as $0.015 \mathrm{mg}$ in $1 \mathrm{~mL}$ of aqueous solution which already suffices when aiming at detecting drugs in street samples. However, the detection of drugs or metabolites in biological samples would require lower detection ranges [3031]. However, giving recent advances in imprinting technology, it is possible to dramatically improve the binding capacity and selectivity of the MIPs in the future. By combining these improved MIPs with high extinction coefficient dyes that are engineered to have very similar structures to the target, it should be possible to bring the sensitivity of the assay into the physiologically relevant concentration regime.

To our knowledge, it is the first time that a MIP sorbent platform has been combined with low-cost dye molecules for the selective detection of illicit drug compounds. The results obtained in this study illustrate that SDC is a highly promising assay for low-cost and fast screening of unidentified powders for the presence of narcotic substances. Due to the generic nature of the synthetic receptors, the range of drug molecules that can be detected by the platform is technically unlimited although the assay has to be optimized depending on the desired target. This universal applicability, in addition to its tuneable 
selectivity and sensitivity, makes the assay advantageous over current low-cost drug test kits. The low cost price and fast reaction time of the assay however, are comparable to current low-cost tests, rendering SDC advantageous in comparison to classical laboratory techniques that are superior in selectivity and sensitivity.

\section{Acknowledgements}

The authors are grateful for funding through the "Limburg Meet" project funded by the province of Limburg, the Netherlands and the Royal Society of Chemistry (Research Fund RF18-2881 and RSC mobility grant). Technical support by numerous Maastricht Science Programme undergrad students is greatly appreciated. 


\section{Chapter 4 References}

[1] Wulff, G. Enzyme-like Catalysis by molecularly imprinted polymers. Chem. Rev. 2002, $102,1-28$.

[2] Sellergen, B.; Allender, C. J. Molecularly imprinted polymers: a bridge to advanced drug delivery. Adv. Drug Del. Rev. 2005, 57, 1733-1741.

[3] Whitcombe, M. J.; Kirsch, N.; Nicholls, I. A. Molecular imprinting science and technology: a survey of the literature for the years 2004-2011. J. Mol. Recogn. 2014, 27, 297401.

[4] Chianella, I.; Guerreiro, A.; Moczko, E.; Gaygill, J. S.; Piletska, E. V.; Perez De Vargas Sansalvador, I. M.; Whitcombe, M. J.; Piletsky, S. A. Direct replacement of antibodies with molecularly imprinted polymer nanoparticles in ELISA - development of a novel assay for vancomycin. Anal. Chem. 2013, 85, 8462-846.

[5] Yano, K.; Karube, I. Molecularly Imprinted Polymers for Biosensor Applications. Trends Anal. Chem. 1999, 18, 199-204.

[6] Haupt, K.; Mosbach, K. Molecularly imprinted polymers and their use in biomimetic sensors. Chem. Rev. 2000, 100, 2495-2504.

[7] Ye, L.; Haupt, K. Molecularly imprinted polymers as antibody and receptor mimics for assays, sensors and drug discovery. Anal. Bioanal. Chem. 2004, 378, 1887-1897.

[8] Wackers, G.; Vandenryt, T.; Cornelis, P.; Kellens, E.; Theolen, R.; De Ceuninck, W.; Losada-perez, P.; van Grinsven, B.; Peeters, M.; Wagner, P. Array formatting of the heattransfer method (HTM) for the detection of small organic molecules by molecularly imprinted polymers. Sensors. 2014, 14, 11016-11030.

[9] King, H. A.; El-Sharif, H. F.; Matia-Gonzalez, A. M.; Ladevaia, V.; Fowotade, A.; Reddy, S. M.; Gerber, A. P. Generation of ribosome imprinted polymers for sensitive detection of translational responses. Sci. Rep. 2017, 7, 6542.

[10] Steen Redeker, E.; Eersels, K.; Akkermans, O.; Royakkers, J.; Dyson, S.; Nurekeyeva, K.; Ferrando, B.; Cornelis, P.; Peeters, M.; Wagner, P.; Dilien, H.; van Grinsven, B.; Cleij, T. J. Biomimetic bacterial identification platform based on thermal wave transport analysis (TWTA) through surface-imprinted polymers. ACS Inf. Dis. 2017, 3, 388-397.

[11] Selvoni, G.; Marrazza, G.; MIP-based sensors: promising new tools for cancer biomarker determination. Sensors. 2017, 17, 718.

[12] Vandenryt, T.; van Grinsven, B.; Eersels, K.; Cornelis, P.; Kholwadia, S.; Cleij, T. J.; Thoelen, R.; De Ceuninck, W.; Peeters, M.; Wagner, P. Single-shot detection of neurotransmitters in whole-blood samples by means of the heat-transfer method in combination with synthetic receptors. Sensors. 2017, 17, 2701.

[13] Fernandes, R.; Dinc, M.; Raimundo Jr, I.; Mizaikoff, B. Molecularly imprinted coreshell hybrid microspheres for the selective extraction of vanillin. Analytical Methods. 2017, 9, 2883-2889.

[14] Diliën, H.; Peeters, M.; Royakkers, J.; Harings, J.; Cornelis, P.; Wagner, P.; Redeker, E.; Banks, C. E.; Eersels, K.; van Grinsven, B.; Cleij, T. J. Label-free detection of small organic molecules by molecularly imprinted polymer functionalized thermocouples: toward in vivo applications. ACS Sens. 2017, 2, 583-589.

[15] Zhao, W.; Zhang, R.; Xu, S.; Cai, J.; Zhu, X.; Zhu, Y.; Wei, W.; Liu, X.; Luo, J. Molecularly imprinted polymeric nanoparticles decorated with Au NPs for highly sensitive and selective glucose detection. Biosens. Bioelectron. 2018, 100, 497-503. 
[16] Canfarotta, F.; Czulak, J.; Betlem, K.; Sachdeva, A.; Eersels, K.; van Grinsven, B.; Cleij, T. J.; Peeters, M. A novel thermal detection method based on molecularly imprinted nanoparticles as recognition elements. Nanoscale. 2018, 10, 2081-2089.

[17] Vlatakis, G.; Andersson, L. I.; Muller, R.; Mosbach, K. Drug assay using antibody mimics made by molecular imprinting. Nature. 1993, 361, 645-647.

[18] Haupt, K.; Dzgoev, A.; Mosbach, K. Assay system for the herbicide 2,4dichlorophenoxyacetic acid using a molecularly imprinted polymer as an artificial recognition element. Anal Chem. 1998, 70, 628-631.

[19] Urraca, J. L.; Moreno-Bondi, M. C.; Orellana, G.; Sellergren, B.; Hall, A. J. Molecularly imprinted polymers as antibody mimics in automated on-line fluorescent competitive assays. Anal Chem. 2017, 79, 4915-4923.

[20] Shi, C.; Liu, X. Y.; Song, L. Y.; Qiao, X. G.; Xu, Z. X. Biomimetic enzyme-linked immunosorbent assay using a hydrophilic molecularly imprinted membrane for recognition and fast determination of trichlorfon and acephate residues in vegetables. Food Anal Meth. 2015, 8, 2496-2503.

[21] Koh, K. N.; Araki, K.; Ikeda, A.; Otsuka, H.; Shinkai, S. Reinvestigation of CalixareneBased Artificial-Signaling Acetylcholine Receptors Useful in Neutral Aqueous (Water/Methanol) Solution. J. Am. Chem. Soc. 1996, 118,755-758.

[22] Tan, J.; Li, R.; Jiang, Z. T. Discrimination of fresh fruit juices by a fluorescent sensor array for carboxylic acids based on molecularly imprinted titania. Food Chem. 2014, 165, $35-41$.

[23] Greene, N. T.; Shimizu, K. D. Colorimetric molecularly imprinted polymer sensor array using dye displacement. J. Am. Chem. Soc. 2005, 127, 5695-5700.

[24] Lee, J. D.; Greene, N. T.; Rushton, G. T.; Shimizu, K. D.; Hong, J. I. Carbohydrate Recognition by Porphyrin-Based Molecularly Imprinted Polymers. Org. Lett. 2007, 7, 963966.

[25] Silverio, O. V.; So, R. C.; Elnar, K. J. S.; Malapit, C. A.; Cristine, M.; Nepomuceno, M. Development of dieldrin, endosulfan, and hexachlorobenzene-imprinted polymers for dye-displacement array sensing. J. Appl. Pol. Sci. 2016, 14, 44401-44411.

[26] Philip, M.; Fu, S. A review of chemical 'spot' tests: A presumptive illicit drug identification technique. Drug Test Anal. 2018, 10, 95-108.

[27] Buchanan, J. F.; Brown C. R. 'Designer drugs'. A problem in clinical toxicology. Med Toxicol Adverse Drug Exp. 1998, 3, 1-17.

[28] Bey, T.; Patel, A. Phencyclidine intoxication and adverse effects: a clinical and pharmacological review of an illicit drug. Cal. J. Emerg. Med. 2007, 8, 9-14.

[29] Castaneto, M. S.; Gorelick, D. A.; Desrosiers, N. A.; Hartman, R. L.; Pirard, S.; Huestis, M. A. Synthetic cannabinoids: epidemiology, pharmacodynamics, and clinical implications. Drug Alcohol Depend. 2014, 1, 12-41.

[30] Kunsman, G. W.; Levine, B.; Constantino, A.; Smith, M. L. Phencyclidine Blood Concentrations in DRE Cases. J Anal Toxicol. 1997, 21, 498-502.

[31] Helander, A.; Beck, O.; Bäckberg, M. Intoxications by the dissociative new psychoactive substances diphenidine and methoxphenidine. Clin. Toxicol. 2015, 53, 446453. 


\section{Supplementary Information}

Table S1. Composition of MIPs synthesized for the imprinting of 2-MXP, with the corresponding imprint factor (IF) against the reference NIP.

\begin{tabular}{|c|c|c|c|c|c|c|}
\hline MIP & $\begin{array}{c}\text { Template } \\
\text { mg (2- } \\
\text { MXP) }\end{array}$ & $\begin{array}{c}\text { MAA } \\
(\mathrm{mL})\end{array}$ & $\begin{array}{c}\text { Crosslinker } \\
(\mathrm{mL})\end{array}$ & Initiator & $\begin{array}{c}\text { DMSO } \\
(\mathrm{mL})\end{array}$ & $\begin{array}{l}\text { IMPRINT } \\
\text { FACTOR }\end{array}$ \\
\hline 20 & 50 & 0.09 & $\begin{array}{c}\text { (EGDMA) } \\
0.35\end{array}$ & $\begin{array}{c}\text { ACVA } \\
50 \mathrm{mg}\end{array}$ & 3 & 1.65 \\
\hline 21 & 50 & 0.09 & $\begin{array}{c}\text { (EGDMA) } \\
0.35\end{array}$ & $\begin{array}{l}\text { ACVA } \\
50 \mathrm{mg}\end{array}$ & 2 & 1.02 \\
\hline 22 & 50 & 0.09 & $\begin{array}{c}\text { (EGDMA) } \\
0.35\end{array}$ & $\begin{array}{l}\text { ACVA } \\
50 \mathrm{mg}\end{array}$ & 4 & 1.32 \\
\hline 23 & 50 & 0.09 & (TRIM) 0.60 & $\begin{array}{l}\text { ACVA } \\
50 \mathrm{mg}\end{array}$ & 3 & 1.01 \\
\hline 24 & 50 & 0.09 & $\begin{array}{c}\text { (EGDMA) } \\
0.35\end{array}$ & $\begin{array}{l}\text { AIBN } \\
50 \mathrm{mg}\end{array}$ & 3 & 2.18 \\
\hline 25 & 50 & 0.09 & (TRIM) 0.60 & $\begin{array}{l}\text { AIBN } \\
50 \mathrm{mg}\end{array}$ & 3 & 1.07 \\
\hline 33 & 50 & 0.07 & $\begin{array}{c}\text { (EGDMA) } \\
0.35\end{array}$ & $\begin{array}{l}\text { AIBN } \\
50 \mathrm{mg}\end{array}$ & 3 & 1.12 \\
\hline 34 & 50 & 0.13 & $\begin{array}{c}\text { (EGDMA) } \\
0.35\end{array}$ & $\begin{array}{l}\text { AIBN } \\
50 \mathrm{mg}\end{array}$ & 3 & 0.9 \\
\hline
\end{tabular}




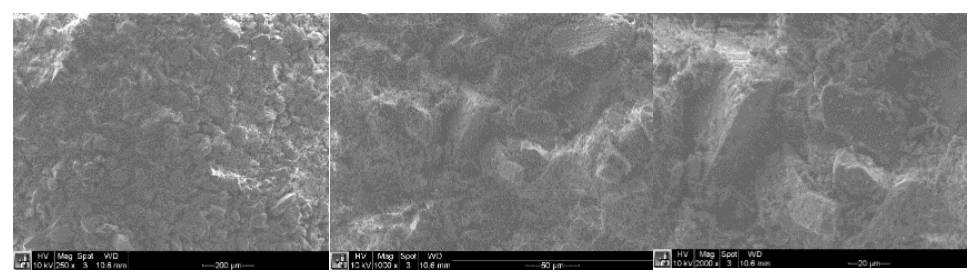

Figure. S2. SEM analysis of MIP particles at 250 (left), 1000 (middle) and 2000X (right) magnification. The typical heterogenous structure of the MIP particles is typical for bulk polymerized MIPs.<smiles></smiles>

(a)<smiles>CN(C)c1ccc(C(=C2C=CC(=[N+](C)C)C=C2)c2ccc(N(C)C)cc2)cc1</smiles>

(d)<smiles>O=C1C=CC(=C(c2ccc(O)cc2)c2ccccc2O)C=C1</smiles>

(b)<smiles>NC1=CCC(=C(c2ccc(N)cc2)c2ccc(N)cc2)C=C1</smiles>

(c)<smiles>CN=C1C=CC(=C(c2ccccc2)c2ccc(N(C)C)cc2)C=C1</smiles>

(e)<smiles>CN(C)c1ccc(N=Nc2ccc(S(=O)(=O)O)cc2)cc1</smiles>

(f)<smiles>COc1ccccc1C(Cc1ccccc1)N1CCCCC1</smiles>

Methoxphenidine hydrochloride salt

Figure S3. Chemical structures of dyes utilized for both rebinding and Substrate Displacement Colorimetry (SDC) in comparison to the structure of 2-methoxphenidine. (a) Basic Blue, (b) Phenol Red, (c) Pararosaline, (d) Malachite green, (e) Crystal Violet, (f) Methyl Orange 


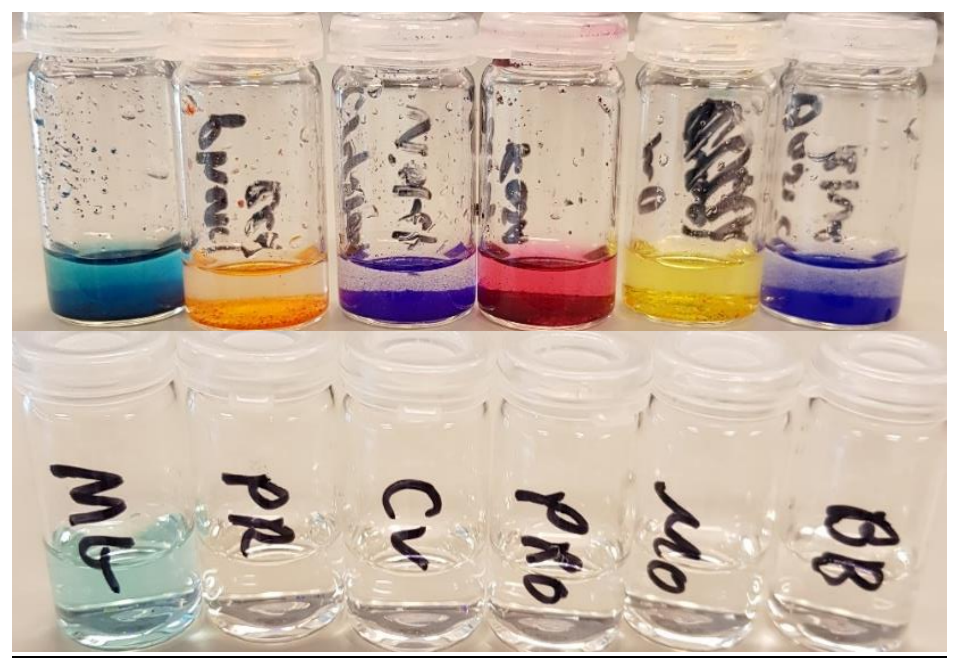

Figure. S4. SDC concept. MIP particles loaded with malachite green, phenol red, crystal violet, pararosaniline, methyl orange and basic blue (left to right) were incubated with 5 $\mathrm{ml}$ 2-MXP (1 mM) for 5 minutes (top). The particles were then filtered off and the resulting filtrates colour observed (bottom).

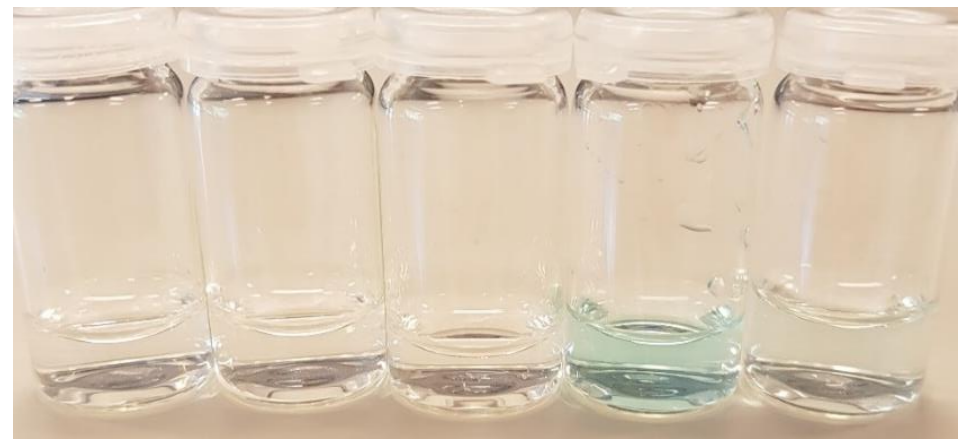

Figure. S5. SDC-concept. MIP-21, MIP-22, MIP-23, MIP-24 and MIP-25 (left to right) were preloaded with malachite green and incubated with $5 \mathrm{ml} 2-\mathrm{MXP}(1 \mathrm{mM})$, filtered and the colour of the filtrate observed. 


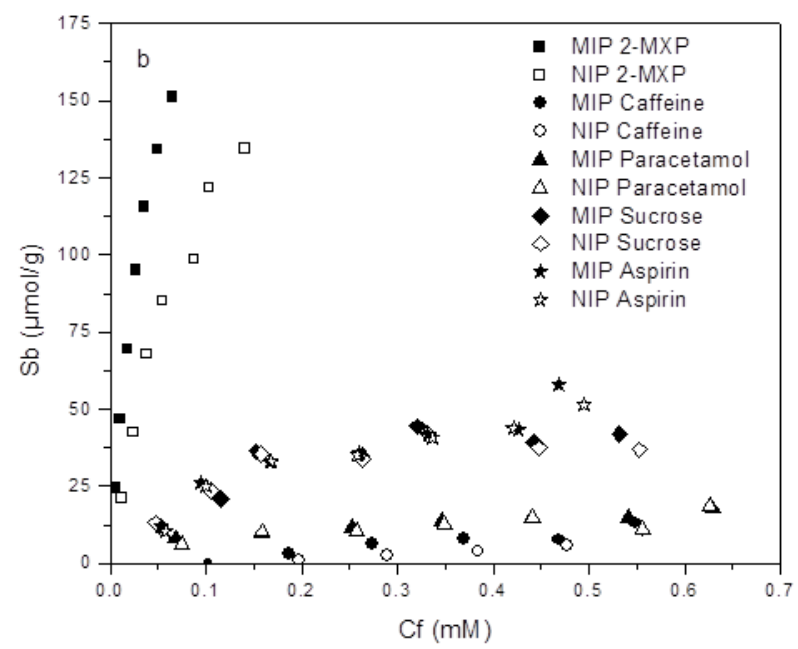

Figure. S6. Selectivity experiment indicating the binding between MIP-24, two common adulterants, and two other pharmaceutically active compounds<smiles>COc1ccccc1C(Cc1ccccc1)N1CCCCC1</smiles>

(a)<smiles>CC(=O)Nc1ccc(O)cc1</smiles>

(b)<smiles>Cn1c(=O)c2c(ncn2C)n(C)c1=O</smiles>

(c)<smiles>CC1(OC2OC(CO)CC(O)C2O)OC(CO)C(O)C1O</smiles>

(d)<smiles>CC(=O)Oc1ccccc1C(=O)O</smiles>

(e)

Figure. S7. Chemical structure of substrates under study: 2-MXP (a), paracetamol (b), caffeine (b), sucrose (d) and aspirin (e). 


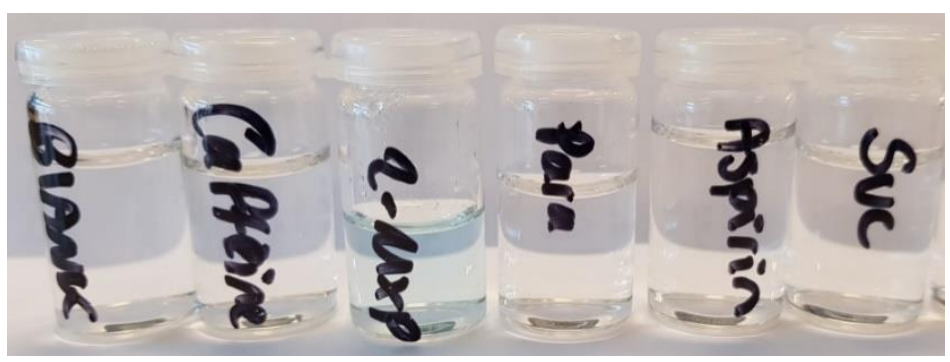

Fig. S8. SDC concept. Malachite-green loaded 2-MXP MIPs were exposed to a range of targets. The filtrate will only color when the MIP is incubated with its target as the dye gets displaced from the MIP particles.<smiles>c1ccc(CC(c2ccccc2)N2CCCCC2)cc1</smiles>

(a)<smiles>CCNC(Cc1ccccc1)c1ccccc1</smiles>

(e)<smiles>COc1ccccc1C(Cc1ccccc1)N1CCCCC1</smiles>

(b)<smiles>CCNC(Cc1ccccc1)c1ccccc1OC</smiles>

(f)<smiles>COc1cccc(C(Cc2ccccc2)N2CCCCC2)c1</smiles>

(c)<smiles>CCNC(Cc1ccccc1)c1cccc(OC)c1</smiles>

(g)<smiles>COc1ccc(C(Cc2ccccc2)N2CCCCC2)cc1</smiles>

(d)<smiles>CCNC(Cc1ccccc1)c1ccc(OC)cc1</smiles>

(h)

Figure. S9. Chemical structure of substrates under study: diphenidine (a), 2-MXP (b), 3MXP (c), 4-MXP (d), ephenidine (e), 2-MEPE (f), 3-MEPE (g) and 4-MEPE (h).

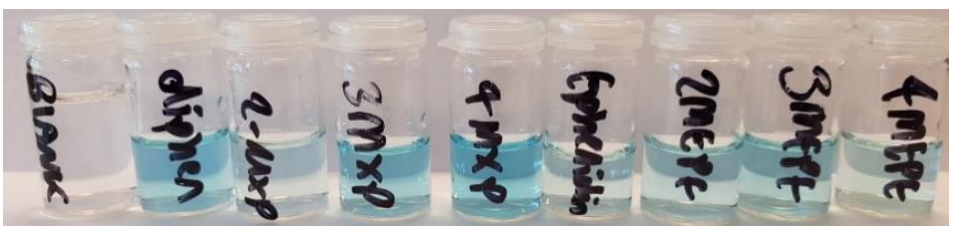

Figure. S10. Malachite-green loaded 2-MXP MIPs were exposed to a range of diarlyethylamines. There is no observable trend in the amount of dye displaced into the filtrate. 


\section{Chapter 5}




\section{MIP based dye displacement assay for amphetamine preface:}

In the previous chapter, it was highlighted how a MIP-based dye displacement assay can be produced by simply pre-loading a dye onto an extracted MIP. The assay developed proved to be easy to use and rapid in response, with an incubation period with the template molecule of only a minute. Some of the other factors that govern the displacement process were investigated, correlating the imprint factor of the MIP with the affinity of the dye being displaced. Thus, enabling a working model to be incorporated to develop such an assay.

The research prior focused on the development of an assay towards the detection of 2-methoxphenidine, being a strong proof-of-principle molecule due to its recent emergence on the illicit substance market and at the time little being known about the compound. The use of this compound in this context demonstrated that new psychoactive substances (NPS) could be easily detected with the novel sensing platform, and leaned into the possibility towards extending the assay format to other compounds.

The research in the next chapter does exactly this, though rather than extending the concept towards other compounds in the NPS field but instead focuses on amphetamine. Thus opening the research line towards the more classical drugs of abuse, expanding the scope and demonstrating how the assay can be tailored to a different chemical architecture. 


\section{Research paper: A Molecularly Imprinted Polymer Based Dye Displacement Assay for the Rapid Visual Detection of Amphetamine in Urine}

Joseph W. Lowdon ${ }^{1}$, Kasper Eersels ${ }^{1}$, Rocio Arreguin-Campos ${ }^{1}$, Manlio Caldara ${ }^{1}$, Benjamin Heidt ${ }^{1}$, Renato Rogosic ${ }^{1}$, Kathia Jimenez Monroy $^{1}$, Thomas J. Cleij ${ }^{1}$, Hanne Diliën ${ }^{1}$, Bart van Grinsven ${ }^{1}$

${ }^{1}$ Sensor Engineering Group, Faculty of Science and Engineering, Maastricht University, PO Box 616, 6200 MD, Maastricht, the Netherlands 


\begin{abstract}
:
Traditionally, the rapid sensing of drug compounds relies upon antibodies, enzymes and electrochemical reactions. However, these technologies can frequently produce false positives/negatives and require specific conditions to operate. A kin to antibodies, molecularly imprinted polymers (MIPs) are a more robust synthetic alternative that has the ability to bind a target molecule with an affinity comparable to that of its natural counterparts. With this in mind, the research presented in this article introduces a facile MIP-based dye displacement assay for the detection of $( \pm)$ amphetamine in urine. The selective nature of MIPs coupled with a displaceable dye enables the resulting low-cost assay to rapidly produce a clear visual confirmation of a targets presence, offering huge commercial potential. The following manuscript characterizes the proposed assay, drawing attention to various facets of the sensor design and thus optimization. To this end, synthesis of a MIP tailored towards amphetamine is described, scrutinizing the composition and selectivity (ibuprofen, naproxen, 2-methoxphenidine, quetiapine) of the reported synthetic receptor. Dye selection for the development of the displacement assay follows, proceeded by optimization of the displacement process by investigating the time taken and the amount of MIP powder required for optimum displacement. An optimized dose-response curve is then presented, introducing $( \pm)$ amphetamine hydrochloride $(0.01-1 \mathrm{mg} \mathrm{mL}-1)$ to the engineered sensor and determining the limit of detection (LoD). The research culminates in the assay being used for the analysis of spiked urine samples (amphetamine, ibuprofen, naproxen, 2-methoxphenidine, quetiapine, bupropion, pheniramine, bromopheniramine) and evaluating its potential as a low-cost, rapid and selective method of analysis.
\end{abstract}

Keywords: Molecularly Imprinted Polymers, Colorimetry, Displacement assay, amphetamine 


\section{Introduction}

Discovered in 1887, Amphetamine has been notoriously a drug of abuse over the decades with its uses varying from a cognitive enhancer, athletics enhancer, treatment for nasal congestion, anti-depressant, euphoriant and even as an aphrodisiac [1-4]. The name Amphetamine commonly refers to both enantiomers being (D) dextroamphetamine and (L) levoamphetamine, with Damphetamine exhibiting a more pronounced effect on the central nervous system (CNS) [5]. This activity and the list of potential uses poses an issue from both a medical and analytical standpoint, giving rise to various challenges that must be overcome. Confirmation of the presence of amphetamine in a hosts urine is an analytical minefield, with current rapid screening methods failing (e.g. immunoassays, ELISA) to discriminate between amphetamine and related compounds such as tricyclic compounds, anti-depressants (quetiapine, bupropion), anti-inflammatory drugs (ibuprofen, naproxen) certain antihistamines (pheniramine, bromopheniramine), nasal inhalers, and even some cold/flu medications [6]. Therefore, developing a test that can easily discriminate between this wide array of compounds is essential. The most prevalent methods of amphetamine detection are conducted on blood and/or urine [7]. Urine analysis has the advantage of being non-invasive and posing no risk to the patient. This also removes the complexities and associated costs that are commonly associated with plasma analysis, and therefore the possibility of detection of amphetamine in urine using molecularly imprinted polymers seems the most interesting route from a commercial point-of-view.

Molecularly Imprinted Polymers (MIPs) have been a rapidly developing field over the last couple of decades, with MIPs demonstrating selective analyte binding affinities on par with that of enzymes [8-12]. In addition to being a valuable asset in separation sciences, catalysis and sensor applications [13-29], MIPs have been incorporated into colorimetric assays in over the years. The conversion of a MIP into a colorimetric tool has many approaches, with various molecular mechanisms being employed to achieve the desired colorimetric verification (quantum dots, dye displacement assays, enzymatic reactions) [3036]. The most straightforward of which was introduce by McNiven et al, synthesizing a dye labelled analogue of chloramphenicol that could be bound to the MIP and displaced in the presence of the parent molecule [37]. It was then demonstrated by Greene et al. that the displaced dye molecule does not have to share a resemblance to the target molecule, opening up the possibility of using a wide range of other dye molecules [38]. Benzofurazan dyes were 
used as an examples, generating a competitive binding assay to detect the presence of six imprinted amine compounds. The concept was then taken further by Lowdon et al. presenting the use of commercially available dyes (malachite green) in a MIP-based dye displacement assay that was coined substrate displacement colorimetry (SDC) [39].

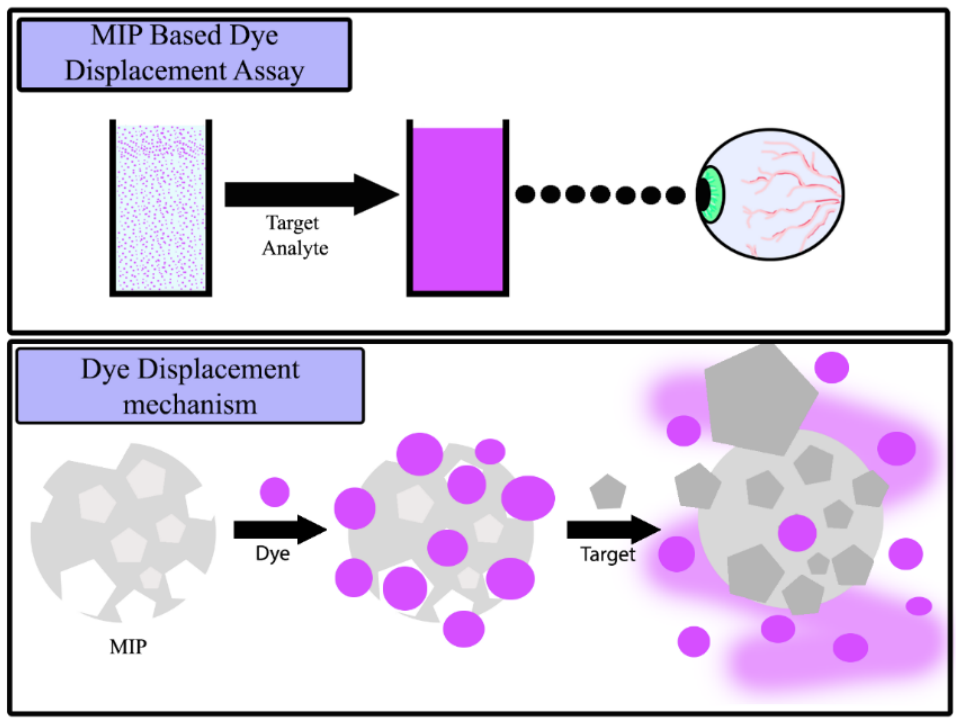

Figure 1. Artistic interpretation of observable colour change when the MIP based dye displacement assay is exposed to a solution containing a target analyte, and an interpretation of the driving force behind the molecular mechanism.

In essence, SDC is a method of converting MIPs into a visual analytical tool by pre-binding a dye molecule into the nanocavities available in the MIP. Upon the addition of the template molecule, the pre-bound dye molecule is released due to the higher affinity of the template molecule towards the complimentary MIP. This process is therefore described as a displacement reaction, as one molecule is replaced on the surface of the MIP by a higher affinity molecule. Compared to other implementations of MIPs into sensing arrays, this MIPbased dye displacement assay offers a fast and straightforward method of gaining rapid visual confirmation of a target analyte (Figure 1). This previous research focuses the technology towards the detection of New Psychoactive 
Substances (NPS), though the methodology would also be useful for the detection of more classical illicit substances such as amphetamine.

In this article, we investigate the possibility of applying this technique towards the detection of amphetamine, generating a rapid colorimetric test for its presence in urine. To this end, a MIP is developed towards the binding of amphetamine and its selectivity is demonstrated in the presence of common molecular interferences. A full study on the binding of various dye molecules is conducted, selecting the optimum dye for the displacement assay. The results collected demonstrate the rapid nature of the generated assay, specifying the optimized time span and amount of polymer powder required for analysis. A dose-response curve illustrates the limit of detection (LoD) of the methodology, building towards the analysis of spiked urine samples for amphetamine.

The research endeavours to highlight how a simple MIP-based assay can be developed towards the detection of an applicable real world target. Thus stressing the benefits of the assay over the more conventional means of analysis, while offering a low-cost alternative in the process. The commercial implications of this technology could be monumental, with the potential of the methodology being developed towards the analysis of a wide range of targets in many sectors. 


\section{Materials and Methods}

\section{Chemicals and reagents}

Prior to polymerization, stabilizers were removed from the functional and crosslinking monomers by passing the reagents over a column packed with alumina. All chemicals and solvents were obtained from Sigma Aldrich (Zwijndrecht, the Netherlands). All tested analyte solutions were prepared with deionized water of resistivity of $18.2 \mathrm{M} \Omega \mathrm{cm}-1$ or with phosphate buffered saline (PBS) solutions unless stated otherwise. License reference 506207WCO, regarding the handling and storage of class 1 compounds within The Netherlands.

\section{Synthesis of molecularly imprinted polymers}

The procedure for the synthesis of the optimized MIP followed the method previously reported [39]. In essence, a mixture of the functional monomer methacrylic acid (MAA, $0.27 \mathrm{ml}, 3.1 \mathrm{mmol}$ ), functional crosslinker ethylene glycol dimethacrylate (EGDMA, $1.73 \mathrm{ml}, 9.2 \mathrm{mmol}$ ), and thermal initiator azobisisobutyronitril (AIBN, $50 \mathrm{mg}, 0.30 \mathrm{mmol}$ ) in $3 \mathrm{ml}$ of dimethyl sulfoxide (DMSO) together with ( \pm ) amphetamine hydrochloride ( $50 \mathrm{mg}, 0.29 \mathrm{mmol}$ ) was prepared in a $15 \mathrm{ml}$ glass vial. The mixture was degassed with N2 before initiating the polymerization reaction. The vial containing the reaction mixture was then placed into an oil bath and heated at $65^{\circ} \mathrm{C}$ for $12 \mathrm{~h}$, allowing for the full completion of the polymerization reaction. The MIP proceeded to be milled several times using a Fritsch Planetary Micro Mill Pulverisette 7 premium line (700 rpm, 3 minutes, $10 \mathrm{~mm}$ balls). After milling, the particles were sieved at $1.0 \mathrm{~mm}$ amplitude using a Fritsch Analysette 3 for 2 hours or until sufficient amount of polymer was on the collection plate to achieve microparticles with sizes smaller than $100 \mu \mathrm{m}$. Finally, the template molecule was removed from the MIP powders by continuous Soxhlet extraction with a 1:10 mixture of acetic acid and ethanol for $12 \mathrm{~h}$, followed by further extraction with pure ethanol for a further $12 \mathrm{~h}$ and drying of the particles at $65^{\circ} \mathrm{C}$ overnight. A reference nonimprinted polymer (NIP) was also prepared in parallel. All MIP compositions test can be found in Supplementary Information Table S1, alongside their corresponding IF values. 


\section{Optical binding experiments}

Optical batch rebinding experiments were evaluated with a Shimadzu UV3600 spectrophotometer. A dilution series $(0-0.7 \mathrm{mM})$ was prepared by varying aliquots of a $1 \mathrm{mM}$ stock solution of amphetamine hydrochloride being added to PBS solution. Subsequently, $20 \mathrm{mg}$ of MIP/NIP powder was added to $5 \mathrm{~mL}$ of each concentration and the resulting suspensions placed on an orbital shaker $(125 \mathrm{rpm})$ for $1 \mathrm{~h}$ at room temperature. After filtration, the free concentration of substrate in the filtrate was determined by UV-vis spectroscopy, allowing the construction of the corresponding binding isotherm. As a measure of specificity and in order to compare the different MIP compositions, the imprint factor (IF) was determined at $\mathrm{Cf}=0.025 \mathrm{mM}$. This method was repeated for each of the MIPs synthesized in this study, alongside a selectivity study where an array of related compounds (ibuprofen, 2methoxphenidine, naproxen, quetiapine, 2-methoxphenidine) binding towards the MIP was tested. Alongside the aforementioned analogues tested, a series of dye molecules (crystal violet, basic blue, pararosaniline, mordant orange and phenol red) had their affinities towards the MIP and NIP tested, identifying the most feasible dye for SDC applications.

\section{Preparation of substrate displacement colorimetry MIP particles}

To $50 \mathrm{ml}$ of aqueous crystal violet $(1 \mathrm{mM})$ was added $1 \mathrm{~g}$ of MIP powder, this was then left to stir $(150 \mathrm{rpm})$ at R.T for one hour allowing the dye molecule to bind to the MIP. The resulting suspension was then collected by means of filtration, and washed rigorously with DI water $(3 \mathrm{~L})$ until the filtrate ran colourless. To ensure there was no leeching of unwanted dye from the MIP, a Shimadzu UV-3600 spectrophotometer measured the UV absorbance of the filtrate collected. The washed dye loaded MIP particles proceeded to dried in an oven at $80^{\circ} \mathrm{C}$ overnight, yielding the dried SDC MIP powder ready for analysis. The same process was complete for the NIP, providing a reference material for comparison in future experiments.

\section{Time dependency of dye Displacement}

SDC MIP particles $(20 \mathrm{mg})$ were incubated with $5 \mathrm{~mL}$ of $( \pm)$ amphetamine hydrochloride $(0.5 \mathrm{mg} \mathrm{mL}-1)$ in PBS (1x), evaluating the amount of dye displaced by means of UV-spectroscopy $(\lambda \max =590 \mathrm{~nm})$ at defined time intervals $(1,2,3,4,5,10,20,30$ minutes). Each time interval was analyzed individually with the SDC MIP particles removed at the specified time interval 
stated. The filtrate was then analyzed with a Shimadzu-3600 UVspectrophotometer $(3.5 \mathrm{~mL}$ quartz cuvette, path length of $1 \mathrm{~cm}$ ), and the maximum absorbance $(\lambda \max =590 \mathrm{~nm})$ noted. Each time interval was repeated in triplicate, with the mean absorbance reported.

\section{Optimisation of the mass of SDC particles for analysis}

( \pm ) Amphetamine hydrochloride in PBS (1x) $(10 \mathrm{~mL}, 0.5 \mathrm{mg} \mathrm{mL}-1)$ was incubated with various masses of SDC MIP particles $(10,15,20,30 \& 40 \mathrm{mg})$ for 1 minute before the filtration of the sample. The remaining solution was then collected and the absorbance of the crystal violet $(\lambda \max =590 \mathrm{~nm})$ assessed by means of a Shimadzu-3600 UV-spectrophotometer (3.5 mL quartz cuvette, path length of $1 \mathrm{~cm}$ ). Each mass of SDC particles in the hydrogel mesh was repeated in triplet, with the average absorbance and standard deviation reported.

\section{SDC Dose-response}

A dose-response study was constructed by incubating the synthesized SDC MIP (30 mg) with varying concentrations of $( \pm$ ) amphetamine hydrochloride (0.01 - $1 \mathrm{mg} \mathrm{mL}-1)$ in PBS (1x) for 1 minute before the flirtation of the sample. The filtrate proceeded to be analyzed by a Shimadzu-3600 UVspectrophotometer $(3.5 \mathrm{~mL}$ quartz cuvette, path length of $1 \mathrm{~cm}$ ) collecting the spectrum for each sample $(350-800 \mathrm{~nm})$. The maximum absorbance of each concentration $(\lambda \max =590 \mathrm{~nm})$ was then plotted against the concentration of $( \pm)$ amphetamine hydrochloride added, allowing the dose response to graphed. The process was then repeated for the NIP, providing a reference for the displacement reaction to be compared against.

\section{Selectivity of the dye displacement}

To study the selectivity of the displacement reaction the SDC MIP was exposed to $5 \mathrm{~mL}$ of various targets $(0.1 \mathrm{mg} \mathrm{mL}-1)$ (ibuprofen, 2-methoxphenidine, naproxen, quetiapine, 2-methoxphenidine) in PBS (1x) before visually assessing the filtrate and collecting the absorbance spectrum.. The process was then repeated for the analysis of each of the compounds in urine (collected from one of the authors, and spiked with each of the specified compounds), enabling a comparison of the assay in a simulated real world sample. Each analysis was repeated in triplet, reporting the average values and standard deviation. 


\section{Results}

\section{Analysis of amphetamine MIPs}

To analyze the binding capabilities of the MIPS synthesized, batch rebinding experiments were undertaken. Known amounts of MIP/NIP powder $(20 \mathrm{mg}$ ) were incubated with increasing concentrations of $( \pm)$ amphetamine hydrochloride $(0.1-0.7 \mathrm{mM})$ for 90 minutes, before measuring the remaining free concentration of target in solution. This was achieved by means of generating a calibration graph for amphetamine and extrapolating the $\lambda$ max (258 nm) absorbance values for the MIP/NIP solutions. Once the free concentration $(\mathrm{Cf}, \mathrm{mM})$ remaining in solution had been confirmed, the amount of target bound to the MIP/NIP per gram ( $\mathrm{Sb}$, $\mu$ mol g-1) was calculated, allowing the construction of the corresponding binding isotherms. This was done for each of the MIP/NIP compositions, with the binding isotherm for the best performing (highest binding capacity, specificity and imprint factor) MIP/NIP being shown below (Figure 2a).
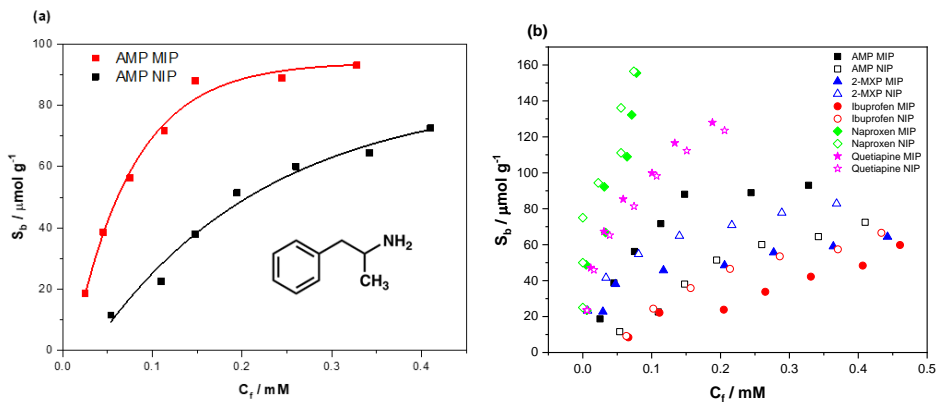

Figure 2. (a) The binding isotherm for MIP-32 (red) and NIP (black) in response to increasing concentrations of $( \pm)$ amphetamine $(0.1-0.7 \mathrm{mM})$ in DI water, and $(\mathrm{b})$ the binding isotherm for MIP-32 in response to compounds that stimulate false positives on current POC drug tests (ibuprofen, 2-MXP, Pheniramine, Bromopheniramine, Naproxen and quetiapine fumarate).

The data was fit using OriginPro8 (OriginLabs Corporation, Northampton, MA, United States) using an allometric $(\mathrm{y}=\mathrm{axb})$ fit, modelling the binding isotherm of both the MIP (red line, R2 = 0.98419) and NIP (black line, R2 = 0.97139). MIP-32 had a maximum binding capacity of $93.13 \mu \mathrm{mol}$ g-1, compared to $72.06 \mu \mathrm{mol} g-1$ of the NIP. 
To evaluate and directly compare each composition the imprint factors (IF) of each MIP/NIP was calculated; with the IF being defined as the amount of target bound by the MIP in comparison to the amount of target bound by the NIP at a given concentration. The concentration this calculation is performed at tends to be low $(\mathrm{Cf}=0.025 \mathrm{mM})$, giving an accurate representation of how the MIP/NIP initially performs under unsaturated binding conditions (See Supplementary Information Table S1). MIP-32, the best performing MIP, therefore had a calculated IF value of 4.4 (at $\mathrm{Cf}=0.025 \mathrm{mM})$.

The selectivity of the MIP towards ( \pm ) amphetamine was then scrutinized, incubating the MIP with compounds that cause current tests to generate false positives (Figure $2 b$ ). The compounds tested included ibuprofen (red circles), 2-MXP (blue triangles), naproxen (green diamonds), and quetiapine fumarate (pink stars). The data for the interaction of the compounds with both MIP and NIP is displayed, indicating whether the binding of each compound can be contributed towards specific or non-specific interactions. To facilitate a direct comparison of the binding the data was allometrically fit ( $y=a x b)$ and the substrate bound $(\mathrm{Sb})$ calculated for each compound for both corresponding $\mathrm{MIP} / \mathrm{NIP}$ at $\mathrm{Cf}=0.025 \mathrm{mM}$ (See Supplementary Information Figure S1). The binding factor $(\mathrm{BF})$ for each compound was then calculated in the same manner as the IF, thus generating a binding factor for each compound that could easily be compared (Table. 1). 
Table 1. The amount of each compound bound to both MIP and NIP $(\mathrm{Sb})$ at $\mathrm{Cf}=0.25 \mathrm{mM}$, and their corresponding binding factors (BF).

\begin{tabular}{|c|c|c|c|c|c|}
\hline Compound & $\begin{array}{c}\mathrm{Sb}\left(\mu \mathrm{mol} \mathrm{g}^{-1}\right) \\
(\mathrm{Cf}=0.025 \\
\mathrm{mM})\end{array}$ & $\begin{array}{c}\text { Binding } \\
\text { Factor } \\
\text { (BF) }\end{array}$ & Dye & $\begin{array}{c}\mathrm{Sb}\left(\mu \mathrm{mol} \mathrm{g} \mathrm{g}^{-1}\right) \\
(\mathrm{Cf}=0.025 \\
\mathrm{mM})\end{array}$ & $\begin{array}{c}\text { Binding } \\
\text { Factor (BF) }\end{array}$ \\
\hline $\begin{array}{c}( \pm) \\
\text { Amphetamine }\end{array}$ & $\begin{array}{l}\text { MIP: } 18.90 \\
\text { NIP: } 4.29\end{array}$ & 4.4 & $\begin{array}{c}\text { Crystal violet } \\
\text { (CV) }\end{array}$ & $\begin{array}{l}\text { MIP: } 48.43 \\
\text { NIP: } 29.29\end{array}$ & 1.7 \\
\hline 2-MXP & $\begin{array}{l}\text { MIP: } 27.67 \\
\text { NIP: } 38.30\end{array}$ & 0.71 & $\begin{array}{c}\text { Mordant } \\
\text { orange (MO) }\end{array}$ & $\begin{array}{l}\text { MIP: } 27.38 \\
\text { NIP: } 32.17\end{array}$ & 0.85 \\
\hline Ibuprofen & $\begin{array}{l}\text { MIP: } 5.77 \\
\text { NIP: } 13.91\end{array}$ & 0.41 & $\begin{array}{l}\text { Basic blue } \\
\text { (BB) }\end{array}$ & $\begin{array}{l}\text { MIP: } 61.82 \\
\text { NIP: } 37.86\end{array}$ & 1.6 \\
\hline Naproxen & $\begin{array}{l}\text { MIP: } 73.33 \\
\text { NIP: } 96.47\end{array}$ & 0.76 & $\begin{array}{l}\text { Phenol red } \\
\text { (PR) }\end{array}$ & $\begin{array}{l}\text { MIP: } 5.89 \\
\text { NIP: } 9.68\end{array}$ & 0.6 \\
\hline Quetiapine & $\begin{array}{l}\text { MIP: } 59.57 \\
\text { NIP: } 52.69\end{array}$ & 1.13 & $\begin{array}{l}\text { Pararosaniline } \\
\text { (PA) }\end{array}$ & $\begin{array}{l}\text { MIP: } 63.26 \\
\text { NIP: } 57.52\end{array}$ & 1.1 \\
\hline
\end{tabular}

The affinity of 2-MXP, ibuprofen and naproxen towards the MIP was less than that of the affinity towards the NIP. Thus suggesting that the NIP offers greater binding modes towards these compounds than the MIP.

\section{Preparation and evaluation of the dye displacement assay}

Evaluation of the binding affinities of dye molecules was conducted in a similar manner to that previously described for the analysis of amphetamine and the analogue compounds above. Though this time crystal violet, basic blue, pararosaniline, mordant orange, and phenol red had their binding capabilities assessed. The data was then fit, plotting the responsives of both the MIP and NIP, giving an idication of which compound had the greatest specific interaction towards the imprinted polymer (Figure 3). Of the dyes tested, crystal violet gave the greatest difference in binding between the MIP and NIP with basic blue being a close second. Basic blue, pararosaniline, crystal violet and mordant orange demonstrated binding capacities with values of $113 \mu \mathrm{mol}$ 
g-1, $102 \mu \mathrm{mol}$ g-1, $76 \mu \mathrm{mol}$ g-1, and $73 \mu \mathrm{mol}$ g-1, with phenol red having the weakest binding capacity of $37 \mu \mathrm{mol} g-1$.

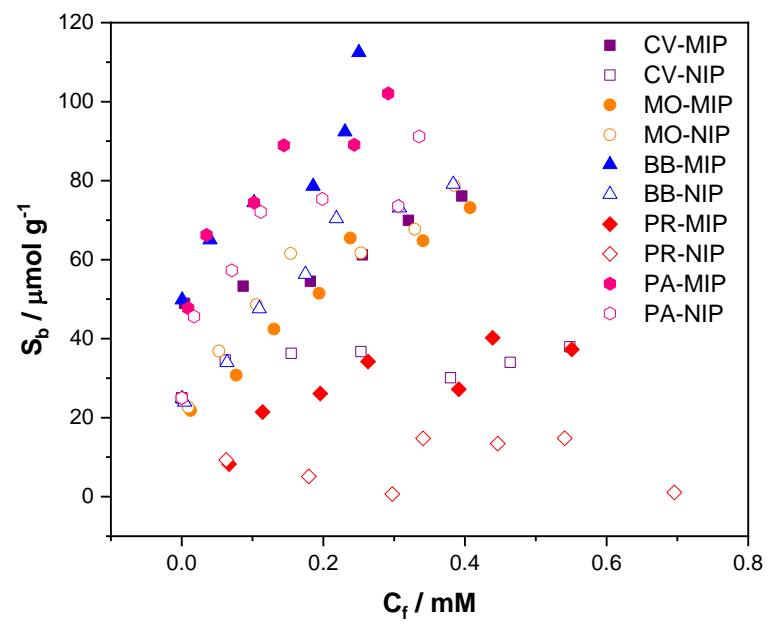

Figure 3. The binding isotherm for MIP-32 in response to varying concentrations of dye molcules (crystal violet - purple squares, mordant orange - orange circles, basic blue blue triangles, pararosaniline - pink hexagons, and phenol red - red diamonds).

To give a metric of how well each dye performed, the binding factor (BF) for each dye was calculated from the fitted data (Table 1). The BF was calculated at the same $\mathrm{Cf}(0.025 \mathrm{mM})$ as mentioned per previous, allowing for the direct comparison in binding. Crystal violet (1.7) provided the highest binding factor with basic blue (1.6) also having a strong specific interaction towards the MIP. The other compounds proved to have either a greater inection towards the NIP (phenol red, and mordant orange) or an interaction of par between the reference and the MIP (pararosanaline).

Of the dyes tested, crystal violet was selected for incubation with the synthesized MIP. Crystal violet demonstrated the highest binding factor towards the MIP/NIP compared to the other dyes tested, demonstrating a high binding capacity alongside the naturally high extinction coefficient of the dye. Before a dose-response curve was conducted, the time for dye displacement to 
occur and the amount of SDC MIP powder required to carry out the reaction was investigated. To this end $20 \mathrm{mg}$ of SDC MIP particles were incubated with aqueous ( \pm ) amphetamine hydrochloride $(0.5 \mathrm{mg} \mathrm{mL}-1)$ and a blank (DI water) for 30 minutes, taking absorbance readings at defined intervals (Figure 4a). There was little to no change in the displacement of the dye after the one minute mark, with the data showing no correlation with the increasing time frame. This was further confirmed visually with a clear consistency in the shade of the dye displaced. The blank demonstrated no leaching of the dye, with the absorbance values remaining around the zero mark.
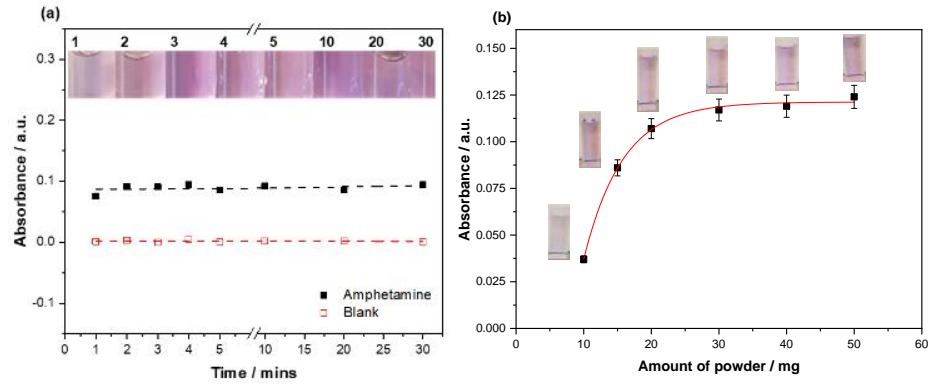

Figure 4. (a) The time depenancy of the dye displacement when $20 \mathrm{mg}$ of SDC MIP powder was in the pressence of $( \pm)$ amphetamine hydrochloride $(0.5 \mathrm{mg} \mathrm{mL}-1$, black squares $)$ and also incubatd with a blank (DI water, red squares), and (b) the effect mass (10 - 50 mg) of dye loaded MIP has on the amount of dye displaced when incubated with $( \pm)$ amphetamine hydrochloride $(0.5 \mathrm{mg} \mathrm{mL}-1)$. The absorbances plotted are that of crystal violet $(\lambda \max =590 \mathrm{~nm})$.

With the time for the displacement reaction to occur explored, scrutiny fell on the mass of SDC MIP and how it impacted the amount of dye displaced. This relationship was investigated by incubating varying masses of SDC MIP (5 $50 \mathrm{mg}$ ) with aqueous $( \pm)$ amphetamine hydrochloride $(0.5 \mathrm{mg} \mathrm{mL}-1)$ and monitoring the absorbance of the dye displaced (Figure $4 \mathrm{~b}$ ). A clear difference in the amount of dye displaced from $5 \mathrm{mg}$ to $30 \mathrm{mg}$ can be seen, though after this point there was little change in the absorbance. This is also visually aparent, with the intensity of the violet colour increasing up until $30 \mathrm{mg}$. the optimized mass was therefore carried forward to the dose-response, alongside the optimized time determined in the previous experiment. 


\section{Dye displacement assay dose response}
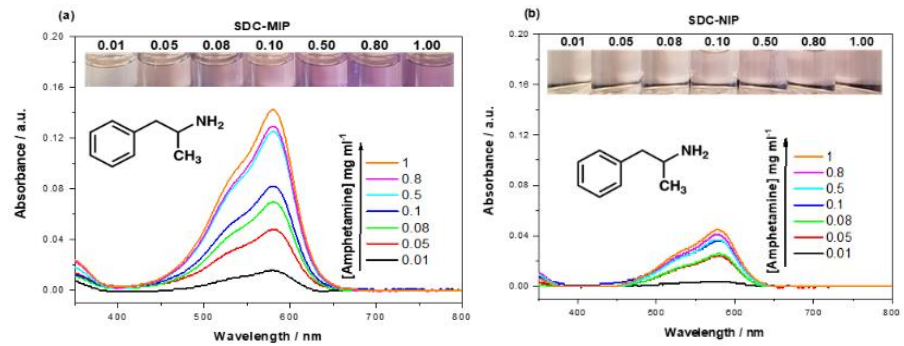

(c)

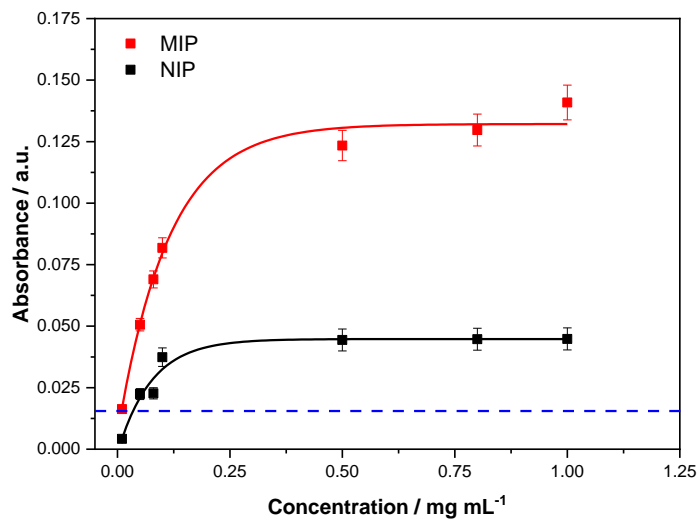

Figure 5. The UV-spectroscopy analysis of crystal violet $(\lambda \max =590 \mathrm{~nm})$ in the filtrates of (a) SDC MIPs and (b) SDC NIPs (30 mg) that were incubated with varying concentrations (0.01 - $1 \mathrm{mg} \mathrm{mL} \mathrm{-1)} \mathrm{of}( \pm)$ amphetamine hydrochloride in water. (c) The comparison of the absorbance $(\lambda \max =590 \mathrm{~nm})$ for the SDC MIP/NIP at the corresponding concentration of amphetamine hydrochloride. Error bars being one standard deviation from the mean reading, and the blue dashed line being the $\mathrm{LoD}(0.009 \mathrm{mg} \mathrm{mL}-1)$ as calculated by the $3 \sigma$ rule.

To ensure the displacement assay performed as expected, the crystal violet loaded SDC MIPs were exposed to varying concentrations of ( \pm ) amphetamine (0.01-1 mg mL-1) and the amount of dye displaced quantised by means of a UV-spectrophotometer (Shimadzu-3600). The absorbance spectra was measured between $350-800 \mathrm{~nm}$, capturing the $\lambda \max (590 \mathrm{~nm}$ ) of crystal violet (Figure 5a, Figure 5b). The absorbance data preceeded to be plotted and fit 
against the concentration of $( \pm)$ amphetamine used using OriginPro8 (OriginLabs Corporation, Northampton, MA, United States) using an allometric $(y=a x b)$ fit, modelling the displacement of dye from both the SDC MIP (red line, R2 = 0.98419) and SDC NIP (black line, R2 = 0.97139) (Figure 5c).

The dye loaded MIP demonstrates a greater displacement than that of the NIP, with the amount of dye displaced being to plateau after $0.5 \mathrm{mg} \mathrm{mL}-1$ amphetamine was introduced. The NIP plateaus a lot sooner with $0.1 \mathrm{mg} \mathrm{mL}$ 1 being the greatest concentration to elicit an inceased dye displacement. Therefore, the displacement from the MIP is observed to be quantitatively and qualitatively greater than that of the NIP, with the filtrate demonstrating a clear darker shade of purple.

\section{Dye displacement selectivity study}
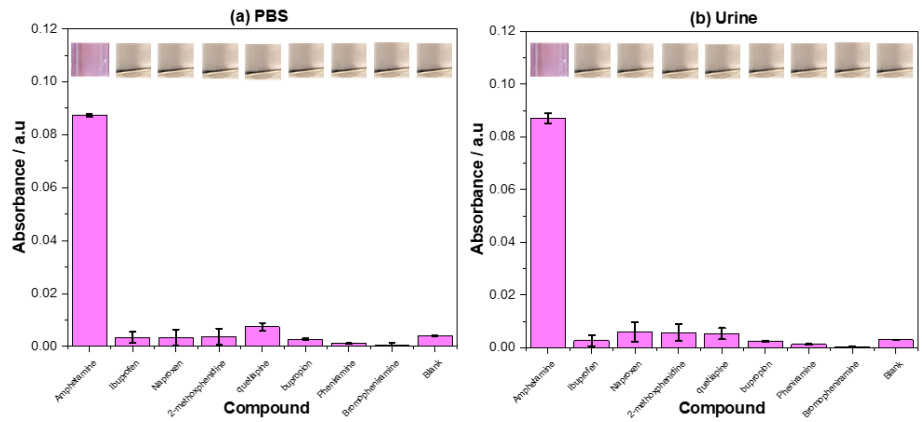

Figure 6. UV-spectrophotometer analysis of the filtrates collected after exposing the dye displacement assay to $( \pm)$ amphetamine, ibuprofen, naproxen, 2-methoxphenidine, quetiapine, bupropion, pheniramine, bromopheniramine $(0.1 \mathrm{mg} \mathrm{mL}-1)$ and a blank in both (a) PBS (1x) and (b) urine. Absorbances were recorded at $\lambda \max =590 \mathrm{~nm}$, reporting the absorbance of crystal violet displaced into the solution.

The selectivity of the assay was analyzed in both PBS and spiked urine, analysing ( \pm ) amphetamine and a host of compounds that stimulate false positives in current tests $( \pm)$ amphetamine, ibuprofen, naproxen, 2methoxphenidine, quetiapine, bupropion, pheniramine, bromopheniramine). The filtrate from the analysis was visually analyzed and the absorbance readings were used to graph the data (Figure 6). 
Of the compounds tested, amphetamine showed the greatest dye displacement when incubated with the assay, performing well in both PBS and in urine. The standard deviation of the dye displaced in PBS was lower than that observed in urine, with the mean value of the dye displaced being practically identical. The other compunds tested in PBS showed little to no dye displacement in comparison to the amphetamine and the blank, with quetiapine demonstrating the greatest dye displacement of the compounds. The same was observed for the compounds in urine, though naproxen and 2-methoxphenidine showed a slightly increased crystal violet concentration in their filtrates. Comparing the PBS based measurements directly to the urine based measurements, as a whole the standard deviation was higher in the urine based measurements with a small increase in the dye displaced by the competitive compounds. 


\section{Discussion}

\section{Evaluation of amphetamine MIP data}

Compared to the other compositions tested, MIP-32 demonstrated the highest binding capacity $(93.13 \mu \mathrm{mol} \mathrm{g}-1)$ in comparison to the corresponding NIP (72.06 $\mu \mathrm{mol} \mathrm{g}-1)$. This fact meant the MIP had the highest calculated IF value of the MIPs analyzed, with an IF value of 4.4. As the IF is a direct numerical representation of the imprinting effect, it indicates that MIP-32 provided the greatest amount of specific binding sites towards $( \pm)$ amphetamine in comparison to its corresponding reference NIP. This is not a surprising result as the other MIP utilized acrylamide and styrene as functional monomers providing little to no binding interactions with the amphetamine. The styrene had potential to form $\pi$-stacking interactions making it a plausible choice of monomer, though these interactions are much weaker than the ionic interactions seen between the methacrylic acid and the amine functionality present in the $( \pm)$ amphetamine molecules. Racemic amphetamine was chosen as a template as this draws parallels to the racemic amphetamine that is present in compounds such as Adderall. Therefore, the MIP is imprinted towards the detection of both enantiomers, though the selectivity towards each individually was not tested. In a real world scenario it is likely that both versions of the compound would be present, and is therefore of higher use to present a binding isotherm depicting the binding of the racemic mixture rather than the individual enantiomers.

Of the compounds determining the selectivity of the MIP, quetiapine was the only compound that demonstrating higher binding towards the MIP than the NIP. This was reflected in the IF values calculated for each of the compounds, with quetiapine having an IF value of 1.13 . The other compounds gave IF values below 1 , indicating the non-specific interactions towards the NIP were stronger than the specific interactions towards the MIP. This is a peculiar result as if you compare the structures of the compounds tested against that of amphetamine (Supplementary Information Figure S2) then quetiapine is one of the least similar structures. The explanation of this binding may lie in the amount of heteroatoms present in the molecule, offer multiple methods of binding to the nanocavities within the MIP. The "tail" of the molecule may have also aided binding, providing easy modes of rotation within the structure and a plausible method of binding to a nanocavity it may have not ordinarily been able to access. The other compounds lacked these characteristics, being 
much larger than amphetamine and having limited binding modes to the cavities expressed in the MIP.

Another explanation for the higher affinity of the compounds towards the NIP than MIP lies in the functionalized space available for the compound to bind to the surface of the polymer. The MIP contains nanocavities that are tailored towards the template molecule, covering the surface with complimentary amphetamine shaped cavities. These cavities may in fact oppose the binding of other molecules, inhibiting or weakening the binding forces that would normally be available. It therefore stands to reason that the NIP offers greater available space for potential binding as it is not littered with complimentary binding sites engineered towards amphetamine, and can therefore provide a larger amount of non-specific interactions. Baggiani et al. have demonstrated this in their work, where they demonstrate that a NIP has to provide a large amount of binding towards molecules for the MIP to also exhibit the same trait [40]. Furthermore, porosity of the MIP has shown to increase the binding of a target molecule, with an increased surface area and therefore amount of binding sites [41]. Thus relating back to the amount of binding sites, and potential areas where other molecules other than the template molecule cannot bind.

\section{Evaluation of dye binding}

Though the dyes tested primarily fell within a tricyclic structure (bar mordant orange), they all interacted differently towards the MIP (Supplementary information Figure S2). Crystal violet proved to be the dye that provided the most specific interaction towards the MIP, followed close by basic blue. This is unexpected as crystal violet contained multiple tertiary amines, whereas pararosaniline containing multiple primary amines and is there closer to the functionalities present in amphetamine. Basic blue also contained multiple tertiary amine functionalities, though has longer $\mathrm{N}$-chain lengths than the methyl groups present in crystal violet. The longer chain lengths would be a plausible reason for the diminished binding capabilities though pararosaniline would be expected to provide the highest binding, based on structure alone. The relative size of the molecules also has on effect on the perceived binding, though basic blue has the much larger structure, followed by crystal violet and final pararosaniline that are similar in size to that of amphetamine. Mordant orange and phenol red provide no specific interaction to the MIP, though this can be attributed to their lack of amine functionality. This speculation is 
strengthened by the research conducted by Dorko et al. characterizing the interactions between different amines architectures and a MIP [42]. Concluding that many factors (including structure and size) affect the binding of a similar molecule to the MIP, but most importantly different factors affect the binding of a compound in different ways. Crystal violet therefore is the dye that was brought forward for the dye displacement assay, demonstrating the highest $\mathrm{BF}$ and a reasonable binding capacity towards the MIP.

\section{Effects on incubation time and mass of SDC MIP}

The amount of the dye displaced by amphetamine was not seen to increase past the period of one minute; this could be due to the difference in binding factor between the dye molecule and the amphetamine. It was shown in previous literature (Lowdon et al. [39]) that the difference in the binding affinities of the molecules was one of the driving forces behind the displacement reaction. Previously, it has been report that the interaction was not as fast as it has been observed here, however the difference in the binding factors in this scenario are much greater than previously reported. The rapidness of the displacement can therefore be attributed to this large difference in binding affinities towards the MIP. This difference in binding affinities might be derived from the differences in size between crystal violet and amphetamine, with crystal violets affinity being dependent on the highly accessible amine functionalities on the edges of the molecule. As there is no perceivable advantage for longer incubation periods, therefore the lower incubation period was brought forward strengthening the argument for the displacement assay being a fast method of analysis and making the assay commercially interesting. The analysis of the blank demonstrated absorbance values of/or around zero, indicating that there was no visible or quantifiable leaching of the dye in solution over the time range analyzed. Again, this is good as it means that the sensor is robust over a longer analysis period.

Optimization of the amount of dye loaded MIP required to carry out analysis revealed that $30 \mathrm{mg}$ was the preferable amount, with the amount dye displaced above the amount only fractionally increased. Below this amount, the dye displaced was greatly reduced and consequently impeded the detection of low concentrations of amphetamine. As the concentration of amphetamine was relatively high $(0.5 \mathrm{mg} \mathrm{mL}-1)$ is made most sense to select a higher mass of the MIP powder, maximizing the possibility of low concentration displacing larger quantities of dye. A saturation effect is observed about the $30 \mathrm{mg}$ indicating 
that there is a point where the amount of the MIP becomes less of a limiting factor than the concentration of the amphetamine presented for incubation.

Considering these factors the overall preparation time of analysis was just a few minutes, including the weighing of the SDC powder, incubation of the sample and filtration of the mixture. This time would be decreased in a real world setting with the amount of SDC-MIP powder already weighed, and a solution could be engineered towards the rapid filtration of the mixture. When compared to other methods of analysis this is still very fast, with other rapid methods taking around 5 minutes (longer for more sensitive methods such as HPLC / mass spectroscopy) [43,44]. This is a distinct commercial benefit as it shows that the MIP based assay can compete in a field where the rapidness of analysis is essential.

\section{Dye displacement dose response evaluation}

The introduction of increasing concentrations of $( \pm)$ amphetamine hydrochloride (0.01 - $1 \mathrm{mg} \mathrm{mL}-1)$ to both dye loaded MIP and NIP yielded dye displacement. The displaced dye for the NIP can be related to the nonspecifically bound dye that is displaced by the amphetamine non-specifically interacting with the polymer. Whereas the displacement of dye from the MIP was much greater than the displacement from the NIP, implying that the dye occupied complimentary nanocavities within the structure. Thus strengthens the argument for the imprinting effect playing a role in the displacement of the dye as it shares some affinity towards the amphetamine specific binding sites. As previously discussed, this may be due to the easily accessible nature of the amine functionalities in the structure of the crystal violet dye being able to bind to the methacrylic acid functionalities inside the nanocavities.

The linear range of the dye displaced from the MIP lies between $0.01-0.20 \mathrm{mg}$ $\mathrm{mL}-1$, with concentrations higher than this showing a diminished capability of dye displacement. Though the linear range is not vast, it supports the analysis of samples at lower concentrations that are more applicable towards physiological concentrations. This said, the lowest observable dye displacement occurs at an amphetamine concentration of $57 \mu \mathrm{M}$, . The LoD was calculated using the $3 \sigma$ rule, being 3 times the maximum standard deviation within the measured samples $(0.009 \mathrm{mg} \mathrm{mL}-1)$. This rule does not take into consideration the dye displaced by the NIP however, which means the LoD falls within the realms of non-specific dye displacement. Typical urine 
concentrations are in the same order of magnitude within the first day upon intake but rapidly fall off in the next day to concentrations that are 100-1000 times lower than the operating regime of the sensor [45]. This indicates that the system still needs to be optimized for regulatory drug abuse testing but still has a lot of commercial potential in the routine screening of unknown solid powder samples. The assay could easily be used in a semi-quantitative capacity for this application, with the intensity of the colour change being a clear reflection of the amount of target present without the need for a UVspectrophotometer to confirm.

\section{Selectivity of the displacement assay}

Scrutiny drawn towards the selectivity of the assay highlighted the capability of the sensing method to selectivity bind amphetamine in both PBS and urine. The selectively was confirmed both visually (purple filtrate) and by means of a UV-spectrophotometer allowing a metric to be related to each of the compounds tested. As observed in the selectivity tests of the raw MIP, the displacement assay demonstrated a low level of dye displacement towards the presence of quetiapine. This can be attributed towards the structural feature previously discussed, enabling the quetiapine to provide a low level of dye displacement. This was seen to be accentuated in PBS, though the displacement was barely greater than that of the blank with it only being noticeable in the metric and not under visual observation. Of the other compounds tested, none demonstrated a displacement much greater (0.005 a.u) than that of the blank, and all paled in comparison to the dye displacement facilitated by the amphetamine. The minor displacement of the dye could prove problematic in the analysis of lower concentrations of amphetamine, though for a rapid on the stop measurement the benefits outweigh the cons. This does not reflect badly on the calculated limit of detection as the value quoted $(0.009 \mathrm{mg} \mathrm{mL}-1)$ falls above the displaced amount of dye. This is a promising result considering that these compounds are common false positives in current tests, and the assay seems to have overcome this issue. The more rigid nature of the polymeric receptors may be the causality of the higher degree of selectivity, hindering the binding of larger molecules. This has huge commercial potential for the assay and it demonstrates the selective nature of the MIPs and how this can be utilized in the displacement assay to produce a result that would normally be impossible in other test formats. 


\section{Conclusions}

Overall, the research highlights how a MIP can be converted into a dye displacement assay that offers huge potential for the rapid analysis of compounds. The assay was shown to work in a model environment (PBS) and in a biofluid (urine) demonstrating the assay's versatility. The selectivity of the assay is superior in comparison to other commercial detection platforms, but is not sensitive enough yet for routine drug testing. This issue can be overcome by employing a more sophisticated method of producing the MIPs in the future. At this point in the research, we wanted to illustrate the commercial potential of the assay and relied upon bulk polymerization as it proved cheap, fast and scalable. A more advanced polymerization protocol, creating MIPs with high binding affinities, in combination with tailor-made dyes with high extinction coefficients could enhance the sensitivity of the assay by multiple orders of magnitude. On the other hand, the current research exemplifies the use of this assay in a laboratory environment for the detection of amphetamine in e.g. unknown powder samples, seized by authorities. This powder would only have to contain a trace amount of amphetamine for the assay to give a positive signal. This finding, in combination with its fast processing time, easeof-use, superior selectivity and low cost-price, indicates that the assay in its current form already has an enormous commercial potential. In addition, with optimization of the sensitivity and widening the application area to the verification of other targets with limited selectivity, the commercial impact could become even bigger in coming years.

\section{Acknowledgments}

The authors are grateful for funding through the "Limburg meet" project funded by the province of Limburg, the Netherlands. Technical support by numerous Maastricht Science Programme undergrad students is greatly appreciated for assistance with analytical equipment and analysis. 


\section{Chapter 5 References}

[1] Colucci, P.; Mancini, C. F.; Santori, A.; Zwergel, C.; Mai, A.; Trezza, V.; Roozendaal, B.; Campolongo, P. Amphetamine and the smart drug 3,4-Methylenedioxypyrovalerone (MDPV) induce generalization of fear memory in rats. Frontiers in Molecular Neuroscience. 2019, 12, 292.

[2] Shin, Y.; Kim, J. Y.; Cheong, J. C.; Kim, J. H.; Kim, J. H.; Lee, H. S. Liquid chromatography-high resolution mass spectrometry for the determination of three cannabinoids, two(-)-trans- $\Delta^{9}$-tetrahydrocannabinol metabolites, and six amphetaminetype stimulants in human hair. J. Chromatography B. 2020, 1149, 122157.

[3] Rozenek, E. B.; Gorska, M.; Wilczynska, K.; Waszkiewicz, N. In search of optimal psychoactivation: stimulants as cognitive performance enhancers. Archives of Industrial Hygiene and Toxicology. 2019, 70(3), 150-159.

[4] Bade, R.; White, J. M.; Nguyen, L.; Tscharke, B. J.; Meuller, J. F.; O’Brien, J. W.; Thomas, K. V.; Gerber, C. Determining changes in new psychoactive substance use in Australia by wastewater analysis. Sci. Total Envi. 2020, 731, 139209.

[5] Phan, D. H.; Shin, E.; Sharma, N.; Hoang, Y.; Tran, P.; Dang, D.; Lee, Y. S.; Lee, Y. J.; Nah, S.; Cheong, J. H.; Jeong, J. H.; Kim, H. 5-HT2A receptor-mediated PKCठ phosphorylation is critical for serotonergic impairments induced by $p$ chloroamphetamine in mice. Food \& Chem Tox. 2020, 141, 111395.

[6] Marin, S. J.; Doyle, K.; Chang, A.; Concheiro-Guisan, M.; Huestis, M. A.; Johnson-Davis, K. L. One Hundred False-Positive Amphetamine Specimens Characterized by Liquid Chromatography Time-of-Flight Mass Spectrometry. J Anal Toxicol. 2016, 40(1), 37-42.

[7] He, Y.; Pohl, J.; Engel, R.; Rothman, L.; Thomas, M. Preparation of ionic liquid based solid-phase microexraction fiber and its application to forensic determination of methamphetamine and amphetamine in human urine. J Chroma A. 2009, 1216, 4824-4830.

[8] Naklua, W.; Suedee, R.; Lieberzeit, P. A. Dopaminergic receptor-ligand binding assay based on molecularly imprinted polymers on quartz crystal microbalance sensors. Biosensors \& Bioelectronics. 2016, 81, 117-124.

[9] Liu, M.; Tran, T. M.; Elhaj, A. A. A.; Torsetnes, S. B.; Jensen, O. N.; Sellergren, B.; Irgum, K. Molecularly Imprinted Porous Monolithic Materials From Melamine-Formaldehyde for Selective Trapping of Phosphopeptides. Anal. Chem. 2017, 89, 9491-9501.

[10] Lowdon, J. W.; Alkirkit, S. M. O.; Mewis, R. E.; Fulton, D.; Banks, C. E.; Sutcliffe, O. B.; Peeters, M. Engineering molecularly imprinted polymers (MIPs) for the selective extraction and quantification of the novel psychoactive substance (NPS) methoxphenidine and its regioisomers. Analyst. 2018, 143, 2081-2089.

[11] Dayal, H.; Ng, W, Y.; Lin, X, H.; Li, S. F. Y. Development of a hydrophilic molecularly imprinted polymer for the detection of hydrophilic targets using quartz crystal microbalance. Sens. Actuators B Chem. 2019, 300, 127044.

[12] Guha, A.; Ahmad, O, S.; Guerreiro, A.; Karim, K.; Sandstrom, N.; Ostanin, V. P.; Wijngaart, W.; Piletsky, S. A.; Ghosh, S. K. Direct detection of small molecules using nanomolecular imprinted polymer receptor and a quartz crystal resonator driven at a fixed frequency and amplitude. Biosensors \& Bioelectronics. 2020, 158, 112176.

[13] Qu, Y.; Qin, L.; Lui, X.; Yang, Y. Reasonable design and sifting of microporous carbon nanosphere-based surface molecularly imprinted polymer for selective removal of phenol from wastewater. Chemosphere. 2020, 251, 126376. 
[14] Pisarev, O. A.; Polyakova. I. V. Molecularly imprinted polymers based on methacrylic acid and ethyleneglycol dimethacrylate for L-lysine recognition. Reactive \& Functional Polymers. 2018, 130, 98-110.

[15] Cantarella, M.; Carroccio, S. C.; Dattilo, S.; Avolio, R.; Castaldo, R.; Puglisi, C.; Privitera, V. Molecularly imprinted polymer for the selective absorption of diclofenac from contaminated water. Chem Eng J. 2019, 367, 180-188.

[16] Vandenryt, T.; van Grinsven, B.; Eersels, K.; Cornelis, P.; Kholwadia, S.; Cleij, T.J.; Thoelen, R.; De Ceuninck, W.; Wagner, P. Single-shot detection of neurotransmitters in whole-blood samples by means of the heat-transfer method in combination with synthetic receptors. Sensors, 2017, 17, 2701.

[17] Eersels, K., Dilien, H., Lowdon, J. W., Redeker, E. S., Rogosic, R., Heidt, B., Peeters, M., Cornelis, P., Lux, P., Reutelingsperger, C. P., Schurgers, L. J., Cleij, T. J., \& Grinsven, B. A novel biomimetic tool for assessing vitamin $\mathrm{K}$ status based on molecularly imprinted polymers. Nutrients. 2018, 10, 751.

[18] Casadio, S.; Lowdon, J. W.; Betlem, K.; Ueta, J. T.; Foster, C. W.; Cleij, T. J.; Grinsven, B.; Sutcliffe, O. B.; Banks, C. E.; Peeters, M. Development of a novel flexible polymer-based biosensor for the thermal detection of noradrenaline in aqueous solutions. Chem Eng J. 2017, 315, 459-468.

[19] Lowdon, J. W.; Eersels, K.; Rogosic, R.; Boonen, T.; Heidt, B.; Diliën, H.; van Grinsven, B.; Cleij, T. J. Surface grafted molecularly imrpinted polymeric receptor layers for thermal detection of the New Psychoactive Substance 2-methoxphenidine. Sens. Actuators B Phys. 2019, 295, 586-595.

[20] Arak, H.; Torshizi, M. A. K.; Hedayati, M.; Rahimi, S. Comparative evaluation of aflatoxin and mineral binding activity of molecular imprinted polymer designed for dummy template using in vitro and in vivo models. Toxicon. 2019, 166. 66-75.

[21] Hammam, M. A.; Wagby, H. A.; El Nashar, R. M. Moxifloxacin hydrochloride electrochemical detection base don newly designed molecularly imprinted polymer. Sensors \& Actuators B: Chem. 2018, 275, 127-136.

[22] Wang, D.; Gao, D.; Huang, Y.; Xu, W.; Xia, Z. Preparation of restricted access molecularly imprinted polymers based fiber for selective solid-phase microextraction of hesperetin and its metabolites in vivo. Talanta. 2019, 202, 392-401.

[23] Haginaka, J.; Nishimura, K.; Kimachi, T.; Inamoto, K.; Takemoto, Y.; Kobayashi, Y. Retention and molecular-recognition mechanisms of molecularly imprinted polymers for promazine derivatives. Telanta. 2019, 205, 120149.

[24] Kumar, N.; Narayanan, N.; Gupta, S. Ultrasonication addicted extraction of chlorpyrifos from honey and brinjal using magnetic molecularly imprinted polymers followed by GLC-ECD analysis. Reactive E Functional Polymers. 2019, 135, 103-112.

[25] Yusof, N. N. M.; Tanioka, E.; Kobayashi, T. Molecularly imprinted polymer particles having coordination hydrogen bonding in covalent-imprinting for efficient recognition towards vanillin. Separation \& Purification Tech. 2014, 122, 341-349.

[26] Sobiech, M.; Maciejewska, D.; Lulinski, P. Synthesis and characterization of poly(methacrylic acid-co-trimethylolpropane trimethacrylate) imprinted sorbent for analysis of biogenic amines. Materials Today Comm. 2020, 22, 100739.

[27] Zhang, Y.; Qin, B.; Zhang, B.; Ma, J.; Hu, Y.; Han, L.; He, M. F.; Liu, C. Specific enrichment of caffeic acid from taraxacum mon-golicum Hand.-Mazz. By $\mathrm{pH}$ and magnetic dual-responsive molecularly imprinted polymers. Analytica Chimica Acta. 2020, 1096, 193-202. 
[28] Mohiuddin, I.; Grover, N.; Aulakh, J. S.; Lee, S.; Malik, A. K.; Kim, K. Porous molecularly-imprinted polymer for detecting diclofenac in aqueous pharmaceutical compounds. Chem Eng J. 2020, 381, 123002.

[29] Hijazi, H. Y.; Bottaro, C. S. Molecularly imprinted polymer thin-film as a microextraction absorbent for selective determination of trace concentrations of polycyclic aromatic sulfur heterocycles in seawater. J Chromatography A. 2020, 1617, 460824.

[30] Zhao, Y.; Ma, Y.; Li, H.; \& Wang, L. Composite QDs@MIP nanospheres for specific recognition and direct fluorescent quantification of pesticides in aqueous medium. Anal. Chem. 2012, 84, 386-395.

[31] Wang, J.; Dai, J.; Xu, Y.; Dai, X.; Zhang, Y.; Shi, W.; Sellergren, B.; \& Pan, C. Molecularly imprinted fluorescent test strip for direct, rapid, and visual dopamine detection in tiny amount of biofluid. Small. 2019, 15, 1803912.

[32] Haupt, K.; Mayes, A. G.; \& Mosbach, K. Herbicide assay using an imprinted polymerbased system analogous to competitive fluoroimmunoassays. Anal. Chem. 1998, 70, 39363939.

[33] Lowdon, J.W.; Diliën, H.; Singla, P.; Peeters, M.; Cleij, T.J.C.; van Grinsven, B.; Eersels, K. MIPs for commercial application in low-cost sensors and assays - An overview of the current status quo. Sens. Actuators B Chem. 2020, 325, 128973.

[34] Li, C.; Ngai, M. H.; Reddy, K. K.; Leong, S. C. Y.; Tong, Y. W.; \& Chai, C. L. L. A fluorescence-displacement assay using molecularly imprinted polymers for the visual, rapid, and sensitive detection of the algal metabolites, geosim and 2-methylisoborneol. Analytica Chimica Acta. 2019, 1066, 121-130.

[35] Silverio, O. V.; So, R. C.; Elnar, K. J. S.; Malapit, C. A.; \& Nepomuceno, M. C. M. Development of dieldrin, endosulfan, and hexachlorobenzene-imprinted polymers for dye-displacement array sensing. J. Appl. Polym. Sci. 2017, 134(2), 44401.

[36] Mattsson, L.; Xu, J.; Preininger, C.; Tse Sum Bui, B.; \& Haupt, K. Competitive fluorescent pseudo-immunoassay exploiting molecularly imprinted polymers for the detection of biogenic amines in fish matrix. Talanta. 2018, 181, 190-196.

[37] McNiven, S.; Kato, M.; Levi, R.; Yano, K.; \& Karube, I. Chloramphenicol sensor based on an in situ imprinted polymer. Analytica Chimica Acta. 1998, 365, 69-74.

[38] Greene, N. T.; \& Shimizu, K. D. Colorimetric molecularly imprinted polymer sensor array using dye displacement. J. Am. Chem. Soc. 2005, 127, 5695-5700.

[39] Lowdon, J. W.; Eersels, K.; Rogosic, R.; Heidt, B.; Diliën, H.; Redeker, E. S.; Peeters, M.; van Grinsiven, B.; Cleij, T. J. Substrate Displacement Colorimetry for the Detection of Diarylethylamines. Sens. Actuators B Chem. 2019, 282, 137-144.

[40] Baggiani, C.; Giovannoli, G.; Anfossi, L. ; Passini, C. ; Baravalle, P. ; \& Giraudi, G. A connection between the binding properties of imprinted and nonimprinted polymers : A change of perspective in molecular imprinting. J. Am. Chem. Soc. 2012, 134(3), 1513-1518.

[41] Krupadam, R.; Venkatesh, A.; \& Piletsky, S., A. Molecularly imprinted polymer receptors for nicotine recognition in biological systems. Molecular imprinting. 2013, 1, 2734 .

[42] Dorko, Z.; Nagy-Szakolczai, A.; Toth, B.; \& Horvai, G. The selectivity of polymers imprinted with amines. Molecules. 2018, 23, 1298.

[43] Shukla, V.; Hussin, F., A.; Hamid, N., H.; \& Ali, N., B., Z. Advances in testing technique for digital microfluidic biochips. Molecules. 2017, 17(8), 1719. 
[44] Stephanson, N., N.; Signell, P.; Helander, A.; \& Black, O. Use of LC-HRMS in full scanXIC mode for multi-analyte urine drug testing - a step towards a "black-box" solution? Mass Spec. 2017, 52(8), 497-506.

[45] Cody, J. T.; \& Valtier, S. Detection of Amphetamine Following Administration of Fenproporex. J. Anal. Tox. 1996, 20(6), 425-431. 


\section{Supplementary Information}

Supplementary Table S1: Chemical compositions of the MIPs tested with their associated imprinting factor (IF) towards amphetamine at $\mathrm{C}_{\mathrm{f}}=0.025 \mathrm{mM}$.

\begin{tabular}{|c|c|c|c|c|c|c|c|c|}
\hline MIP & $\begin{array}{l}\text { MAA } \\
(\mathrm{mM})\end{array}$ & $\begin{array}{c}\mathrm{AA} \\
(\mathrm{mM})\end{array}$ & $\begin{array}{l}\text { Styrene } \\
\text { (mM) }\end{array}$ & $\begin{array}{c}\text { EGDMA } \\
(\mathrm{mM})\end{array}$ & $\begin{array}{l}\text { DMSO } \\
\text { (mM) }\end{array}$ & $\begin{array}{l}\text { AIBN } \\
(\mathrm{mM})\end{array}$ & $\begin{array}{l}\text { Amphetamine } \\
\text { Hydrochloride } \\
\text { (mM) }\end{array}$ & $\begin{array}{c}\text { IF } \\
\left(C_{f}=\right. \\
0.025 \\
m M)\end{array}$ \\
\hline 31 & 3.1 & - & - & 9,2 & 3 & 0.3 & 0.29 & 0.99 \\
\hline 32 & - & 3.1 & - & 9.2 & 3 & 0.3 & 0.29 & 4.4 \\
\hline 33 & - & - & 3,1 & 9.2 & 3 & 0.3 & 0.29 & 0.74 \\
\hline
\end{tabular}

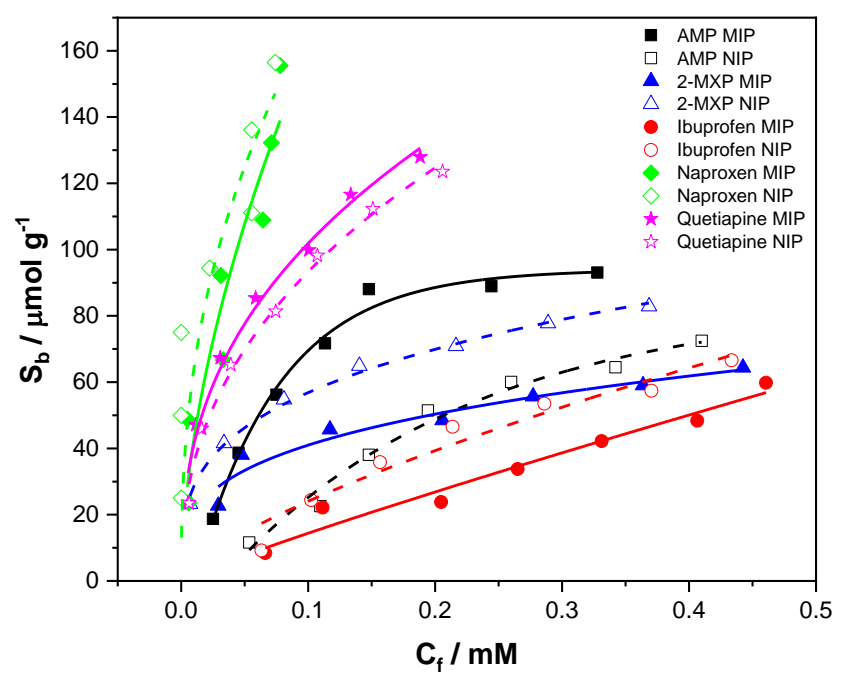

Supplementary Figure S1. The fitted selectivity binding isotherm for MIP-32, demonstrating the binding of amphetamine (black squares), 2-MXP (blue triangles), ibuprofen (red circles), naproxen (green diamonds), and quetiapine (pink stars). 

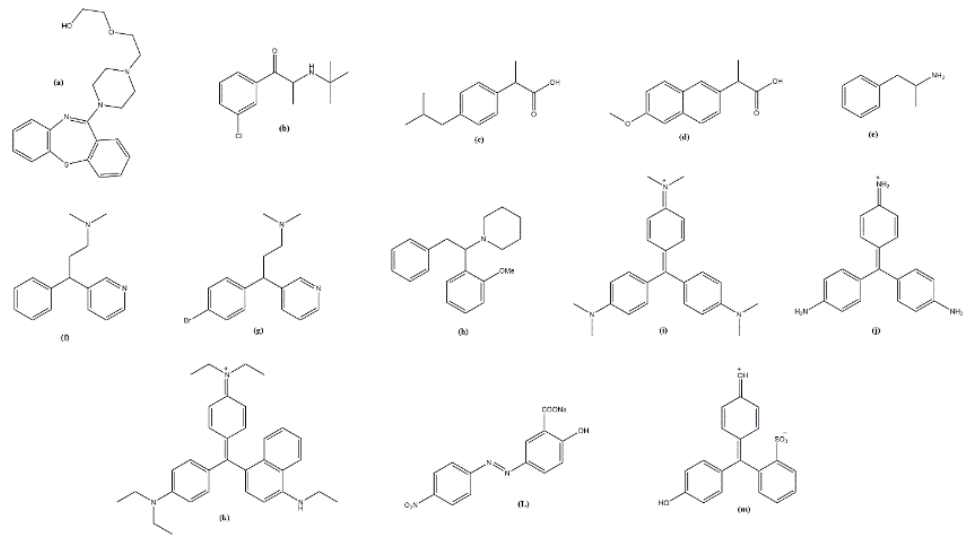

Supplementary Figure S2. The chemical structures of (a) quetiapine, (b) bupropion,(c) ibuprofen, (d) naproxen, (e) amphetamine, (f) pheniramine, (g) bromopheniramine, (h) 2methoxphenidine, (i) Crystal violet, (j) pararosaniline, (k) basic blue, (l) mordant orange, and $(\mathrm{m})$ phenol red. 


\section{Chapter 6}




\section{Creating a dye displacement assay for the detection of antibiotics preface:}

The rapid detection of illicit substances is not the only field that can benefit from the development of low-cost, rapid colorimetric assays. The field of analysing unknown samples is vast, and a large proportion of this sector focuses on the analysis of environmental samples. These samples tend to be screened for pollutants that have potential to harm the environment, or nutrient levels to sustain the environment and allow nature to flourish. It would therefore be beneficial to have an assay that can rapidly relay vital concentrations of a chosen compound/contaminant in real time to a user who is performing analysis of environmental samples. The overall purpose being a more rapid result and the removal of unnecessary lab analysis that is also costly.

One environmental pollutant that has increased over the late $20^{\text {th }}$ century and early $21^{\text {st }}$ century is the presence of antibiotics. These compounds, though helpful for fighting bacterial infections in human and animals alike, have caused detrimental damage to the environment and have led to the raise of "superbugs" (bacteria that are resistant to antibiotics). This causality makes them a strong candidate for the development of an assay specifically targeting their detection. Rapid sample analysis could prevent contaminated samples containing antibiotics reaching the environment, reducing the harm these compounds are causing and increasing the longevity of antibacterial compounds current in circulation.

This research presented in the next section focuses on the development of such an assay, applying the methods previously discussed in the prior chapters. Unlike in the previously described work, a different polymerization approach was adopted (emulsion) yielding more homogenous particles in both size and shape. This in combination with intuitive monomer and dye selection is proven to produce a colorimetric assay capable of detecting aminopenicillins. 


\section{Research paper: Colorimetric Sensing of Amoxicillin Facilitated by Molecularly Imprinted Polymers}

Joseph W. Lowdon 1, Hanne Diliën 1, Bart van Grinsven 1, Kasper Eersels ${ }^{1}$, and Thomas J. Cleij ${ }^{1}$

${ }^{1}$ Sensor Engineering Department, Faculty of Science and Engineering, Maastricht University, P.O. Box 616, 6200 MD Maastricht, The Netherlands

Polymers. 2021, 13(13), 2221. 


\begin{abstract}
:
The scope of the presented research orientates itself towards the development of a Molecularly Imprinted Polymer (MIP) based dye displacement assay for the colorimetric detection of the antibiotic amoxicillin in aqueous medium. With this in mind, the initial development of a MIP capable of such a task sets focus on bulk polymerization to assess monomer/crosslinker combinations that have potential towards the binding of amoxicillin. The best performing composition (based on specificity and binding capacity) is utilized in the synthesis of MIP particles by emulsion polymerization, yielding particles that prove to be more homogenous in size and morphology compared to that of the bulk synthesized MIP, which is essential when it comes to the accuracy of the resulting assay. The specificity and selectivity of the emulsion MIP proceeds to be highlighted, demonstrating a higher affinity towards amoxicillin compared to other compounds of the aminopenicillin class (ampicillin and cloxacillin). Conversion of the polymeric receptor is then undertaken, identifying a suitable dye for the displacement assay by means of binding experiments with malachite green, crystal violet and mordant orange. Once identified, the optimal dye is then loaded onto the synthetic receptor, and the displaceability of the dye deduced by means of a dose response experiment. Yielding a dye displacement assay that can be used (semi-)quantitatively.
\end{abstract}

Keywords: amoxicillin, emulsion polymerization, molecularly imprinted polymers, colorimetry 


\section{Introduction}

Since the discovery of modern-day antibiotics in the early 20th century, these miracle molecules have gone from wonder drug to one of the world's largest environmental contaminants [1]. Over the last couple of decades, the consumption and use of antibiotics has increased, not just by humans but also by cattle [2]. One of the biggest consumers of antibiotics is China, with a reported use of $162,000 \mathrm{t}$ of antibiotics in 2013, with more than half administered to animals [3]. It is common practice to supplement animal feed with antibiotics, ensuring the health and welfare of the creature by reducing the risk of illness or facilitating the rapid recovery from bacterial ailments [4]. Though this is beneficial for the animal in question, it generates an environmental concern. Incomplete metabolism of the antibiotics leads to 30$90 \%$ of the active compounds being excreted by the animal, and in the process introducing the antibacterial agents to their surrounding environment $[5,6]$. Thus, the concentrations of antibiotics in the environment is increasing, and the bacteria that are exposed consistently to these compounds become resistant to their destructive effects. This is just one concern related to the over-use of antibiotics in animals, another is the transfer of antibiotics in animal related byproducts e.g. milk [7]. This makes the detection of antibiotics in both animal waste and in by-products essential, as both the environment and animals (humans) are at risk due to the potential of stimulating and further progressing antibiotic-resistance.

Molecularly imprinted polymers (MIPs) are a developing technology that has shown promise in the field of sensing of low molecular weight compounds, and have demonstrated great potential in the analysis of samples contaminated with antibiotics [8, 9]. MIPs are synthetic polymeric receptors containing nanocavities that have been specifically tailored towards the binding of a chosen molecule, making them the artificial equivalent to antibodies [10-12]. The benefit over their biological counterparts lies in the stability of the receptors, demonstrating high resistance to harsh physical conditions such as $\mathrm{pH}$ and temperature while retaining sensitivity [13,]. This makes them ideal for the analysis of matrices that are outside the normal physiological conditions (wastewater, soil samples) that would normally render the biological receptors useless. To this end, MIPs have been developed in the analysis of antibiotics in the environment, coupling the synthetic receptors to various transducer (e.g. Quartz Crystal Microbalance (QCM), thermal methods, and fluorescent probes) translating binding events at the surface of 
the MIP into a tangible quantifiable signal [14-19]. Though these technologies for the analysis and sensing of antibiotics in complex samples are rapidly progressing, these readout techniques still require a level of expertise to conduct (though great efforts have been made to increase the portability of devices and to make them as user-friendly as possible). A more simplistic MIPbased variation is possible, developing the MIP into a dye displacement assay for the rapid visual verification of a target's presence [20].

The use of MIPs in conjunction with dyes was first conceived in 1998 by McNiven et al. They used a dye-conjugated analogue of chloramphenicol in a competitive capacity to detect the presence of the parent compound [21]. Introduction of the parent compound to the system would displace the dyeconjugate in a quantitative manner, yielding higher displacement of the conjugate in direct correlation to the amount of chloramphenicol present. This concept would take many other forms over the next two decades, modifying template molecules to include chromophores and fluorophores, introducing optical properties into the structures of the MIPs, and demonstrating that the structure of a pre-bound signaling molecule could share no relation to the desired target analyte to be displaced [22-25]. The premise was recently extended further by using commercially available dyes such as malachite green and crystal violet, developing MIP-based dye displacement assay for the detection of diarylethylamines and amphetamine [26, 27]. The research highlighted how low-cost visual sensors can be engineered from MIP technology, though for relatively simple molecules with few competing functionalities. Aminopenicillins however are not simple molecules.

Showing a diverse molecular structure, aminopenicillins encompass a broad range of structural derivatives of ampicillin (which itself is an amino benzylpenicillin) (Figure. 1) [28, 29]. The multiple functionalities within the molecules gives rise to the possibility of different binding opportunities, meaning the selection of functional monomers/crosslinkers is not as definitive as for simpler mono-functional molecules [30]. Therefore, a degree of rational design is required, analyzing the potential binding groups and selecting compositions that not only interact with one functional but with multiple [31]. The same principles can be applied to the selection of a dye for conversion of a MIP into a dye displacement assay. Enabling a comparison of the key functionalities between dye molecule and template molecule, thus optimizing the binding and displacement of the dye. However, optimized ratios and 
chemical interactions are only one part of the story, as further optimization maybe found the polymerization method employed to synthesize said MIPs.<smiles>CN1[C@@H](C(=O)O)[C@H](NC(=O)[C@@H](N)c2ccc(O)cc2)SC1(C)C</smiles>

Amoxicillin

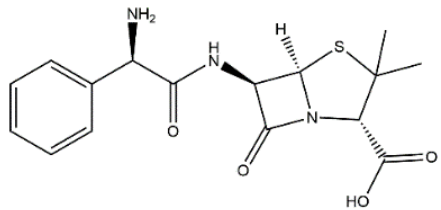

Ampicillin

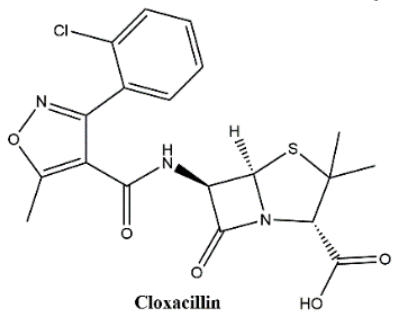

Figure 1. The chemical structures of amoxicillin, ampicillin and cloxacillin

The most common method of producing MIPs is monolithic bulk imprinting, where all components of a MIP are placed into a single container and polymerized [32]. The resulting monolith can be crushed and the template extracted, yielding a powder with a crude morphology [33]. This protocol is robust and can enable the rapid testing of polymer formulations when attempting to design a MIP [34]. Although the bulk imprinting approach is a tried-and-tested approach of successfully producing MIPs, the methodology has been surpassed by more elegant approaches that enable more homogenous morphologies and particles sizes [35-40]. One such methodology is emulsion polymerization, where two phases (aqueous and organic) are introduced in the presence of a surfactant that enables the formation of uniformly sized micelles [41]. These act as micro-reactors containing the polymerization mixture, which in turn enables the formation of more homogenous morphologies. Though more elegant, this kind of methodology lacks in simplicity when compared to its more crude predecessor.

With all this in mind, this research builds upon prior literature, taking optimized monomer/crosslinker ratios and expanding these ratios towards novel compositions for the binding of amoxicillin. Monolithic bulk imprinting is used to rapidly test these compositions, before synthesized MIPs with higher 
homogeneity by an emulsion based approach. The resulting MIP is compared to its monolithic bulk imprinted counterpart, comparing imprint factors at different free concentrations of target analyte. Scrutiny is drawn towards the selectivity of the emulsion made MIP, before determining the binding of a variety of dyes (malachite green, crystal violet, and mordant orange 1) that could potentially be used in the dye displacement assay format. The dye selection being based on the chemical functionalities they have in common with amoxicillin, but also how they interact with the functionalities present in the MIP. Finally, the best performing dye is loaded onto the emulsion MIP by means of incubation and the assay format analyzed. To this end dose response and selectivity tests are conducted, determining the sensitivity of the assay towards amoxicillin and seeing how the assay fairs in the presence of other antibiotics of the same class (cloxacillin and ampicillin). Overall, the research highlights how pragmatic compositional choices, alongside intelligent dye selection can be used to produce a MIP that can be easily developed into a semi-quantitative rapid colorimetric assay format.

\section{Materials and Methods}

\section{Chemicals and equipment}

Acrylamide (AA, 99.5\%, CAS: 79-06-1), Methacrylic acid (MAA, 99.5\%, CAS: 79-41-4), ethylene glycol dimethacrylate (EGDMA, 99.5\%, CAS: 97-90-5), trimethylolpropane trimethacrylate (TRIM, CAS: 3290-92-4), dimethyl sulfoxide (DMSO, reagent grade, CAS: 67-68-5), Malachite green oxylate salt (99.8\%, CAS: 2437-29-8), crystal violet (99.8\%, CAS: 548-62-9), mordant orange 1 (70\%, CAS: 2243-76-7), aluminum oxide (basic, CAS: 1344-28-1), amoxicillin (99.8\%, CAS: 26787-78-0), cloxacillin (99.8\%, CAS: 61-72-3), and ampicillin sodium salt (99.5\%, CAS: 69-52-3) were purchased from Sigma-Merck. Prior to polymerization, all stabilizers were removed from monomers and crosslinkers by passing the compounds over aluminum oxide (basic), thus removing any chemical source that could inhibit the polymerization process. Equipment utilized in the experimentation include a Shimadzu-3600 UVSpectrophotometer ( $3.5 \mathrm{~mL}$ quartz cuvette, path length of $1 \mathrm{~cm}$ ), BlueWave 200 UV light source, Leica Microsystem DM300, and a Shimadzu MIRacle10 FT-IR spectrometer equipped with a Zn/Se ATR crystal over an optical range of 4,000 $\mathrm{cm}^{-1}$ to $450 \mathrm{~cm}^{-1}$. 


\section{Generalised monolithic bulk imprinting method}

The component ratios used for the MIPs synthesized were based on previous literature regarding the monolithic polymerization of molecularly imprinted polymers [42]. In general, functional monomer $(1.4 \mathrm{mmol})$, template $(0.35$ $\mathrm{mmol})$, crosslinker $(2.8 \mathrm{mmol})$ and DMSO $(3.3 \mathrm{~mL})$ were placed in a glass vial and dissolved $\mathrm{O}_{2}$ purged from the mixture by bubbling $\mathrm{N}_{2}$ through the solution for 30 seconds. AIBN ( $36.1 \mathrm{mg}, 0.22 \mathrm{mmol}$ ) was then added to the resulting mixture, ensuring that the solution was thoroughly mixed before subjecting the solution to a UV lamp (BlueWave 200, wavelength: 300-450 nm), thus initiating polymerization under photochemical conditions. The polymeric mixture was exposed to the UV lamp for 30 minutes, before yielding a solid polymer block. The resulting monolith proceeded to be mechanically ground with a ball mill ( 3 cycles, $300 \mathrm{rpm}$ for 180 seconds, 60 second pause between each cycle), producing a fine powder with an average particle size less than 150 $\mu \mathrm{m}$ (confirmed by microscopy). After mechanical grinding, the powder was subject to continuous soxhlet extraction with ethanol/acetic acid (100:1) for 24 hours, before further extraction with ethanol for 12 hours and drying the powder. The same process was completed excluding the template molecule generating a non-imprinted polymer (NIP) that would act as a reference material. The composition of the MIPs/NIPs synthesized can be found in Table 1.

Table 1. MIP/NIP compositions

\begin{tabular}{l|l|l|l|l|l|l} 
MIP/NIP & Methodology & Monomer & Crosslinker & Initiator & Solvent & Template \\
\hline MIP 201 & Bulk & AA & TRIM & AIBN & DMSO & Amoxicillin \\
NIP 201 & Bulk & AA & TRIM & AIBN & DMSO & - \\
MIP 202 & Bulk & MAA & TRIM & AIBN & DMSO & Amoxicillin \\
NIP 202 & Bulk & MAA & TRIM & AIBN & DMSO & - \\
MIP 203 & Bulk & MAA & EGDMA & AIBN & DMSO & Amoxicillin \\
NIP 203 & Bulk & MAA & EGDMA & AIBN & DMSO & - \\
MIP 204 & Bulk & AA & EGDMA & AIBN & DMSO & Amoxicillin \\
NIP 204 & Bulk & AA & EGDMA & AIBN & DMSO & - \\
MIP 205 & Emulsion & MAA & EGDMA & AIBN & DMSO/H2O & Amoxicillin \\
NIP 205 & Emulsion & MAA & EGDMA & AIBN & DMSO/H2O & -
\end{tabular}




\section{Generalized MIP synthesis by emulsion polymerization}

An aqueous phase was prepared by mixing $300 \mathrm{mg}$ of sodium dodecyl sulphate (SDS) in $60 \mathrm{~mL}$ of DI (deionized) water and placing it into a $100 \mathrm{~mL}$ round bottom flask. An overhead stirrer was then placed into the flask, with the stirrer blade positioned so that it was below the surface of the solution. Proceeding this, the organic phase of the reaction was prepared by mixing monomer (MAA/AA), EGDMA, AIBN, amoxicillin and DMSO (compositions in Table 1) in a vial before purging both solutions with $\mathrm{N}_{2}$ for 30 seconds. Once purged, the organic phase was introduced dropwise to the aqueous phase while being vigorously stirred between $600-1400 \mathrm{rpm}$ for 1 minute and enabling an emulsion to form (see Figure 2). Stirring was ceased, and the emulsion exposed to UV light (BlueWave 200) for 1 hour. After the exposure, the solution was transferred to centrifuge tubes and spun at $4500 \mathrm{rpm}$ for 5 minutes. The resulting polymeric disk was left in the centrifuge tube while carefully removing the supernatant and introducing ethanol to the tube. The tube was shaken well suspending the polymeric powder and the centrifugation process repeated for five washes with ethanol. Once fully washed the polymeric powder was transferred into a glass vial and oven-dried at $65^{\circ} \mathrm{C}$ for 12 hours. Thus yielding a fine polymeric powder with a defined particle size based on the rpm initially used to mix the reaction. A non-imprinted reference version of the polymeric composition was synthesized with the exact same methodology described but without the presence of the template molecule. Template extraction was monitored with FTIR comparing the non-extracted MIP, the extracted MIP, and the amoxicillin spectra. Particle sizes were analyzed by optical microscopy (Leica Microsystem DM300) while the $1 \mathrm{mg}$ $\mathrm{ml}^{-1}$ of polymer particles were suspended in water. 


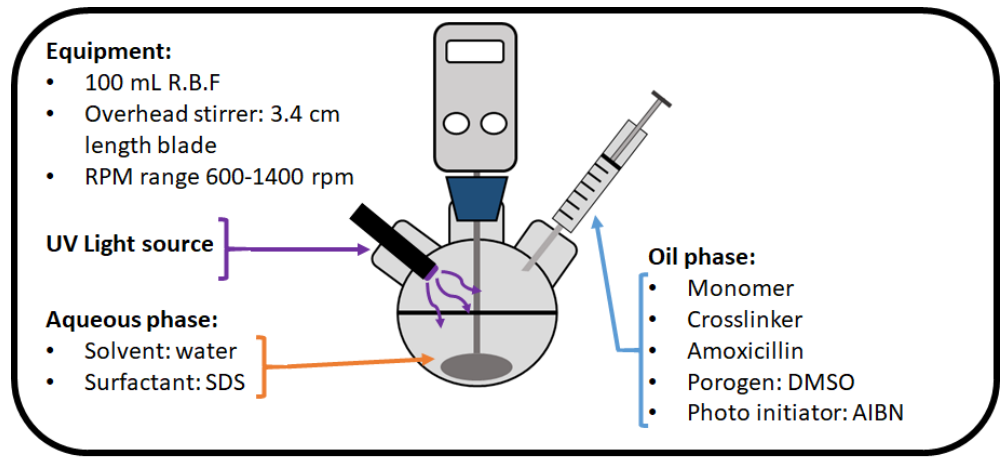

Figure 2. Apparatus schematic for the emulsion polymerization approach of synthesizing MIPs/NIPs with more uniform particle sizes.

\section{Generalized batch rebinding experiment}

To determine the binding affinities of the MIP/NIP, rebinding experiments were conducted as follows: to $20 \mathrm{mg}$ of MIP/NIP powder was added $5 \mathrm{~mL}$ aliquots of aqueous target/analogue ranging in concentration $(0.1-0.7 \mathrm{mM})$ and the resulting suspension agitated on a rocking table (125 rpm) for 90 minutes. After agitation, the filtrate of each sample was collected and analyzed using a UV-spectrophotometer, analyzing the $\lambda$ max of the remaining molecular species in solution $\left(\mathrm{C}_{\mathrm{f}}\right)$. The amount of molecular species bound to the MIP/NIP $(\mathrm{Sb})$ proceeded to be calculated from these observed values and the corresponding binding isotherm plotted. This process was repeated for each of the following species: Amoxicillin, ampicillin, cloxacillin, malachite green, crystal violet, and mordant orange 1.

Note: During the experimentation with malachite green, crystal violet and mordant orange all samples were diluted (dilution factor 100), ensuring absorbance values within the reliable range on the UV-spectrophotometer.

\section{Preloading of dye to MIPs}

$1 \mathrm{~g}$ of MIP 205 powder was added to $50 \mathrm{~mL}$ of aqueous mordant orange $(1 \mathrm{mM})$. The resulting suspension was well stirred for half an hour before transferring the suspension into a centrifuge tube. The solution was then centrifuged at $4500 \mathrm{rpm}$ for 5 minutes, enabling the safe removal of the supernatant while 
allowing the MIP pellet to remain at the bottom of the tube. Proceeding this, 45 $\mathrm{mL}$ of DI water was added to the tube, the vessel sealed, and the tube shaken thoroughly facilitating the dispersal of the MIP powder once more. The centrifugation process was then repeated and the water changed until the resulting supernatant appeared colourless and showed no signs of a residual dye absorbance band by means of UV-spectrophotometry.

\section{Dose response of dye displacement}

A dose-response study was constructed by incubating the synthesized dye loaded MIP 205 (40 mg) with $5 \mathrm{~mL}$ of aqueous amoxicillin at varying concentrations $\left(0.000-365.0 \mathrm{mg} \mathrm{L}^{-1}\right)$ for 1 minute before the filtration of the sample. The filtrate proceeded to be analyzed by a UV-spectrophotometer collecting the spectrum for each sample (200 - $500 \mathrm{~nm})$. The maximum absorbance of each concentration $\left(\lambda_{\max }=385 \mathrm{~nm}\right.$ ) was then plotted against the concentration of amoxicillin added, allowing the dose response to graphed. The same process was repeated for the assay in the presence of cloxacillin and ampicillin, testing the selectivity of the assay. 


\section{Results}

\section{Analysis of bulk MIPs}

Four initial MIP compositions were selected for the binding of amoxicillin after identifying the amine and carboxylic acid functionalities as ideal groups for possible ionic and hydrogen bonding interactions. The four compositions consisted of either methacrylic acid (being complimentary to the amine functionality present in amoxicillin), Acrylamide (complimentary to the carboxylic acid functionality present in amoxicillin), and either EGDMA or TRIM as crosslinker (EGDMA producing a more flexible structure, and TRIM a more rigid network). The binding properties of the MIPs were tested by means of batch rebinding experimentation, where the MIPs were exposed to increasing concentrations $(0.1-0.7 \mathrm{mM})$ of amoxicillin for a defined time (90 mins). After the incubation period the MIPs were removed from the solutions and the remaining concentration of amoxicillin in solution determined $\left(\mathrm{C}_{\mathrm{f}}\right)$, thus the amount of bound amoxicillin to the MIP could be realized $\left(\mathrm{S}_{\mathrm{b}}\right)$. These values were then plotted against each other, yielding the binding isotherm for each MIP composition (Figure 3).

The plotted data points were then fit using an allometric function ( $y=a x b$ ) factoring in the morphology of the polymeric structure and therefore surface area, simulating a saturation effect as higher concentrations of molecular species are introduced to the MIP/NIP. MIP $201-203$ clearly demonstrate this saturation effect towards the higher concentrations, whereas MIP 204 shows no correlation. Of the MIPs tested MIP 202 has the highest perceived binding

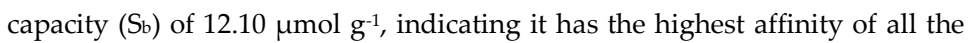
MIPs tested towards amoxicillin. MIP 201 and MIP 203 have slightly lower binding capacities of $10.72 \mu \mathrm{mol} \mathrm{g}-1$ and $9.47 \mu \mathrm{mol} \mathrm{g}-1$ respectively, whereas MIP 04 has an incalculable binding capacity and no feasible trend exists within the data points collected. 

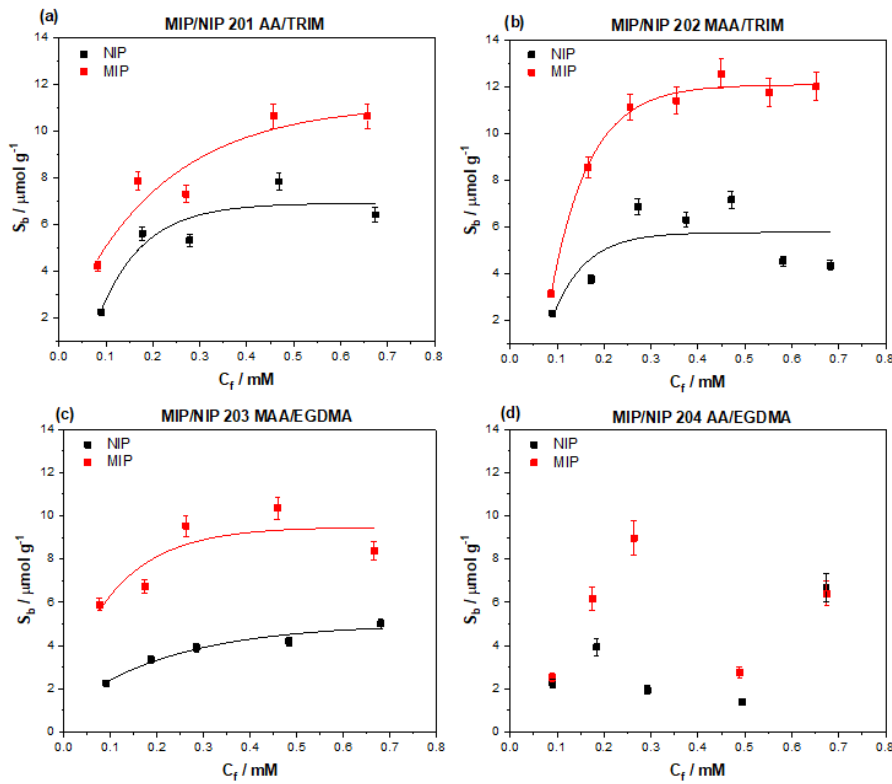

Figure 3. Binding isotherms for the varying MIP compositions (a) AA/TRIM, (b) MAA/TRIM, (c) MAA/EGDMA, and (d) AA/EGDMA synthesized by monolithic free radical polymerization.

A metric was placed on how specific the binding interactions are between the MIP and target molecule by comparing the $\mathrm{S}_{\mathrm{b}}$ of the MIP and NIP at a defined free concentration (in this case $C_{f}=0.1,0.2$, and $0.3 \mathrm{mM}$ ), yielding a value known as the imprint factor (IF) describing the specific interactions of the MIP with the target (Table 2). The MIP with the highest amount of specific interaction was MIP 203, demonstrating IF values of $2.55\left(C_{f}=0.1 \mathrm{mM}\right), 2.48\left(C_{f}\right.$ $=0.2 \mathrm{mM})$, and $2.33\left(\mathrm{C}_{\mathrm{f}}=0.3 \mathrm{mM}\right)$. The IF values calculated for MIP 201 and 202 across this range are diminished in comparison, with MIP 201 having the lowest specific interaction out of the two. The performance of MIP 202 is observed to increase over the calculated concentration range, though the generated IF values are still lower than those calculated for MIP 203. 
Table 2. Binding capacities ( $\mathrm{Sb}$ ) for MIP 201, 202, 203, and 204 at $\mathrm{C}_{\mathrm{f}}=0.1,0.2$ and $0.3 \mathrm{mM}$, and their corresponding imprint factors (IF).

\begin{tabular}{|c|c|c|c|c|c|c|}
\hline MIP/NIP & $\begin{array}{c}\mathrm{S}_{\mathrm{b}} / \mu \mathrm{mol} \\
\mathrm{g}^{-1}(\text { at Cf } \\
=0.1 \\
\mathrm{mM})\end{array}$ & $\begin{array}{l}\text { IF (at } C_{f} \\
=0.1 \\
\mathrm{mM})\end{array}$ & $\begin{array}{c}\mathrm{S}_{\mathrm{b}} / \\
\mu \mathrm{mol} \mathrm{g}^{-1} \\
\left(\text { at } \mathrm{C}_{\mathrm{f}}=\right. \\
0.2 \mathrm{mM})\end{array}$ & $\begin{array}{l}\text { IF (at } C_{f} \\
=0.2 \\
\mathrm{mM})\end{array}$ & $\begin{array}{c}\mathrm{S}_{\mathrm{b}} / \\
\mu \mathrm{mol} \mathrm{g}^{-1} \\
\left(\text { at } \mathrm{C}_{\mathrm{f}}=\right. \\
0.3 \mathrm{mM})\end{array}$ & $\begin{array}{c}\text { IF (at } C_{f} \\
=0.3 \\
\mathrm{mM})\end{array}$ \\
\hline 201 & $\begin{array}{l}\text { MIP } 5.07 \\
\text { NIP } 2.82\end{array}$ & 1.80 & $\begin{array}{l}\text { MIP } \\
7.29 \\
\text { NIP } \\
5.38\end{array}$ & 1.35 & $\begin{array}{c}\text { MIP } \\
8.80 \\
\text { NIP } 6.41\end{array}$ & 1.37 \\
\hline 202 & $\begin{array}{l}\text { MIP } 4.33 \\
\text { NIP } 2.65\end{array}$ & 1.63 & $\begin{array}{l}\text { MIP } \\
9.74 \\
\text { NIP } \\
5.00\end{array}$ & 1.95 & $\begin{array}{c}\text { MIP } \\
11.36 \\
\text { NIP } 5.62\end{array}$ & 2.02 \\
\hline 203 & $\begin{array}{l}\text { MIP } 6.36 \\
\text { NIP } 2.49\end{array}$ & 2.55 & $\begin{array}{l}\text { MIP } \\
8.11 \\
\text { NIP } \\
3.27\end{array}$ & 2.48 & $\begin{array}{c}\text { MIP } \\
8.93 \\
\text { NIP } 3.83\end{array}$ & 2.33 \\
\hline 204 & N/A & N/A & N/A & N/A & N/A & N/A \\
\hline
\end{tabular}

Following the binding analysis of the MIPs, the morphology of the particles was brought into question. Optical microscope images of the particles were taken allowing an assessment of the homogeneity of particles sizes and shapes to be conducted (Figure 4). The images reveal the morphology of the particles is inconsistent and shows a large degree of heterogeneity; therefore, each particle has differing surface area and different potential to interact with molecular species. 


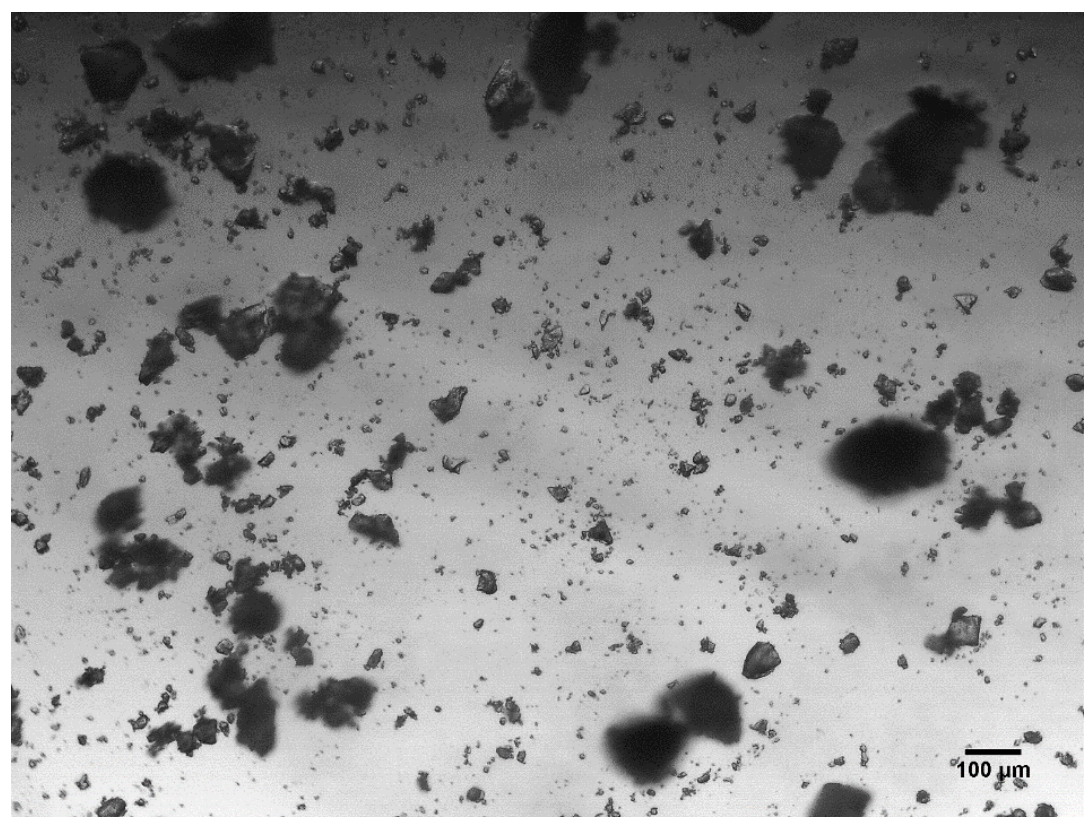

Figure 4. Optical microscope images of finely ground bulk monolith particles below 150 $\mu \mathrm{m}$ in size.

\section{Emulsion Particle Size Analysis}

In an attempt to generate more homogenous MIP particle sizes, an emulsion polymerization approach was adopted. To this end, the most promising monomer and crosslinker combination from the bulk experiments was brought forward (MIP 203), incorporating the same stoichiometric ratios in the emulsion polymerization process. The methodology entailed the addition of an organic phase (monomer, template, crosslinker, porogen and initiator) to an aqueous phase (water and surfactant), thoroughly mixing the two phases to produce an emulsion that could yield finer more homogenous particle sizes (further details in discussion). The degree to which the two phases were mixed was investigated, relating particle size to the speed of the stirring invoked (Figure 5a). Plotting the average particle size produced against the speed (rpm) at which the overhead stirrer was set revealed that the slower $(600 \mathrm{rpm})$ produced larger particles with a higher standard deviation in size $(50 \pm 19 \mu \mathrm{m})$ 
that at the higher speeds. As the stirring surpassed $1000 \mathrm{rpm}$ the particle sizes achieved began to plateau, reaching an average particle size of $8-10 \mu \mathrm{m}$ with a standard deviation range of $\pm 3-4 \mu \mathrm{m}$. The relationship between the lower standard deviation in relation to the speed of stirring is strengthened by the analysis of the particle size distribution (Figure 5b), which indicates the lower speeds produce a wider spread of particle sizes compared to higher speeds producing a narrower band of particle sizes.
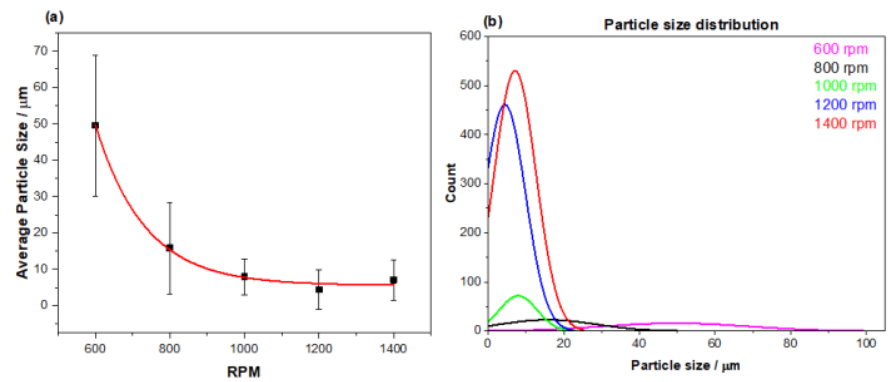

Figure 5. How the stirring speed effects the particle size generated during the emulsion polymerization process with (a) relating the average achieved particle sizes with the rpm used (error bars being the \pm 1 standard deviation in the size measurements), and (b) the distribution of the particle sizes at each speed.

Alongside the particle size distributions, the morphology of the particles at each stirring speed was also assessed (Figure 6). Though the size of the particles may differ with the varying speeds, the overall morphology of the particles remained consistent throughout. Each emulsion produced spherical morphologies with very little deviation in structure apart from the size differences observed. 


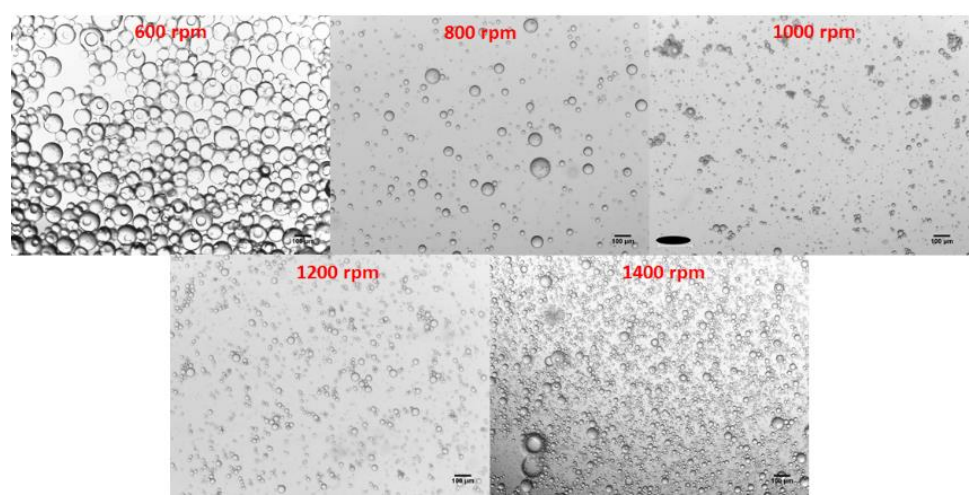

Figure 6. Optical microscope (Leica Microsystem DM300) images of particles sizes produced by varying the stirring speed during the emulsion polymerization process.

\section{Emulsion MIP binding analysis}

To ensure the washing procedure successfully removed the template amoxicillin, an FTIR comparison of the extracted MIP was compared to that of the non-extracted MIP and amoxicillin (Figure 7). The amoxicillin has very distinctive peaks between $2500-3500 \mathrm{~cm}^{-1}(\mathrm{OH}$ stretch) that are not present in the extracted MIP, but are present in the non-extracted MIP. Other key regions that relate to the structure of amoxicillin (e.g. sharp peaks between $1680 \mathrm{~cm}^{-1}$ ( $\mathrm{C}=\mathrm{O}$, amide), $1390-1310 \mathrm{~cm}^{-1}$ (O-H bending, phenol) and below $900 \mathrm{~cm}^{-1}$ ) are not present in the analysis of the MIP, therefore it can be said with confidence that amoxicillin is no longer present in the extracted imprinted polymer. Comparing non-extracted with extracted, the sulfoxide peak $(\mathrm{S}=\mathrm{O}, 1070-1030$ $\mathrm{cm}^{-1}$ ) is seen to disappear, suggesting the washing step is also successful in removing unwanted porogen from the polymer. 


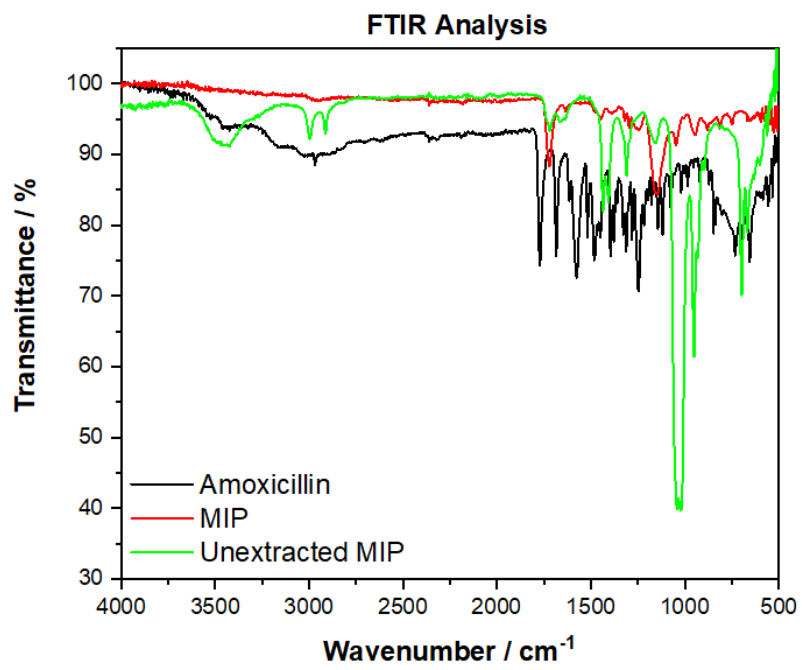

Figure 7. FTIR analysis of amoxicillin (black line), unextracted MIP (green line), and the extracted emulsion MIP (red line), demonstrating that amoxicillin was successfully removed from the imprinted material.

After ensuring the template molecule was fully extracted from the MIP, the same rebinding procedure was followed as with the bulk generated MIP particles. The MIP 205 particles were incubated with increasing concentrations of amoxicillin, analyzing the remaining amount of amoxicillin left in solution after the binding process. Thus, the amount of template bound to the MIP ( $\mathrm{Sb}$ ) was plotted against the remaining free concentration $\left(\mathrm{C}_{\mathrm{f}}\right)$ of amoxicillin in solution (Figure 8a). The data was fit using OriginPro8 (OriginLabs Corporation, Northampton, MA, United States) using an allometric $\left(y=a x^{b}\right)$ fit, modelling the binding isotherm of both the MIP (red line, $\mathrm{R}^{2}=0.84058$ ) and NIP (black line, $\mathrm{R}^{2}=0.9173$ ). 

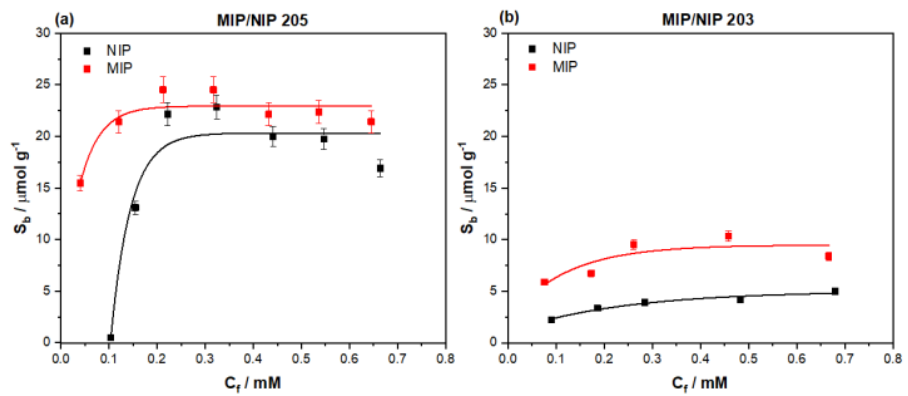

Figure 8. Binding isotherms for (a) MIP 205 prepared by emulsion polymerization and (b) a rescaled MIP 203 prepared by bulk polymerization strategies. Where both MIP (red squares) and NIP (black squares) were plotted and fit.

This data was directly compared to the binding isotherm of the monolithic bulk polymerized MIP 203, rescaling the binding isotherm's y-axis ( $\mathrm{Sb}$ ) to enable the direct comparison of the features of the isotherms (Figure $8 \mathrm{~b}$ ). The emulsion MIP demonstrated a higher overall binding capacity of $22.53 \mu \mathrm{mol} \mathrm{g}-1$ once saturation (plateauing) is observed, comparatively, the monolithic MIP only demonstrated a binding capacity of $9.89 \mu \mathrm{mol} \mathrm{g}{ }^{-1}$. At lower $\mathrm{C}_{\mathrm{f}}$ values $(0.05-0.1$ $\mathrm{mM}$ ) the emulsion prepared MIP 205 particles exhibit a high affinity to the amoxicillin with little to no interaction with the corresponding NIP, unlike the monolithic bulk NIP where binding at the lower concentrations is apparent. MIP 203 consequently performed consistently throughout the concentration range, showing a steady trend of binding the amoxicillin in a similar manner at both high and low concentrations in comparison with the NIP.

For a more direct comparison of the specific nature of the MIPs the imprint factor (IF) was calculated for MIP 205 and compared to that of MIP 203 at multiple free concentrations (Table 3). The comparison of this metric shows that MIP 205 interacts vastly more specifically at this low concentration compared to MIP 203. MIP 205 has a calculated IF $\left(C_{f}=0.1 \mathrm{mM}\right)$ of 45.46 compared to that of MIP 203 that has an IF $=2.55\left(C_{f}=0.1 \mathrm{mM}\right)$. However, the performance of MIP 205 in comparison to the NIP rapidly decreases towards higher free concentrations, with the IF falling to 1.24 and 1.14 at $C_{f}=0.2 \mathrm{mM}$ and $C_{f}=0.3 \mathrm{mM}$ respectively. The performance of MIP 203 on the other hand remains relatively constant (if not a little diminished) throughout the 
concentration range, yielding a calculated IF of $2.48\left(\mathrm{C}_{\mathrm{f}}=0.2 \mathrm{mM}\right)$ and $2.33\left(\mathrm{C}_{\mathrm{f}}\right.$ $=0.3 \mathrm{mM})$.

Table 3. Binding capacities ( $\mathrm{S}_{\mathrm{b}}$ ) for MIP 203 (amoxicillin, ampicillin, and cloxacillin) and MIP 205 at $C_{f}=0.1,0.2$, and $0.3 \mathrm{mM}$, and their corresponding imprint factors (IF).

\begin{tabular}{|c|c|c|c|c|c|c|c|}
\hline MIP & Compound & $\begin{array}{l}\mathrm{Sb} / \\
\mu \mathrm{mol} g \\
{ }^{1} \text { (at Cf } \\
=0.1 \\
\mathrm{mM})\end{array}$ & $\begin{array}{l}\text { IF (at } \\
C_{f}=0.1 \\
m M)\end{array}$ & $\begin{array}{l}\mathrm{S}_{\mathrm{b}} / \\
\mu \mathrm{mol} \\
\mathrm{g}^{-1}(\mathrm{at} \\
\mathrm{C}_{\mathrm{f}}= \\
0.2 \\
\mathrm{mM})\end{array}$ & $\begin{array}{l}\text { IF (at } \\
C_{f}= \\
0.2 \\
m M)\end{array}$ & $\begin{array}{l}\mathrm{S}_{\mathrm{b}} / \\
\mu \mathrm{mol} \\
\mathrm{g}^{-1}(\mathrm{at} \\
\mathrm{C}_{\mathrm{f}}= \\
0.3 \\
\mathrm{mM})\end{array}$ & $\begin{array}{l}\text { IF (at } \\
C_{f}= \\
0.3 \\
m M)\end{array}$ \\
\hline 203 & Amoxicillin & $\begin{array}{l}\text { MIP } 6.36 \\
\text { NIP } 2.49\end{array}$ & 2.55 & $\begin{array}{l}\text { MIP } \\
8.11 \\
\\
\text { NIP } \\
3.27\end{array}$ & 2.48 & $\begin{array}{l}\text { MIP } \\
8.93 \\
\\
\text { NIP } \\
3.83\end{array}$ & 2.33 \\
\hline 205 & Amoxicillin & $\begin{array}{c}\text { MIP } \\
21.45 \\
\text { NIP } 0.47\end{array}$ & 45.64 & $\begin{array}{c}\text { MIP } \\
22.75 \\
\\
\text { NIP } \\
18.34\end{array}$ & 1.24 & $\begin{array}{c}\text { MIP } \\
22.86 \\
\\
\text { NIP } \\
20.10\end{array}$ & 1.14 \\
\hline 205 & Ampicillin & $\begin{array}{l}\text { MIP } 0.39 \\
\text { NIP } 0.28\end{array}$ & 1.39 & $\begin{array}{l}\text { MIP } \\
2.28 \\
\\
\text { NIP } \\
0.21\end{array}$ & 10.86 & $\begin{array}{l}\text { MIP } \\
2.28 \\
\\
\text { NIP } \\
0.48\end{array}$ & 4.75 \\
\hline 205 & Cloxacillin & $\begin{array}{l}\text { MIP } 8.58 \\
\text { NIP } 7.80\end{array}$ & 1.1 & $\begin{array}{c}\text { MIP } \\
10.97 \\
\\
\text { NIP } \\
7.79\end{array}$ & 1.41 & $\begin{array}{c}\text { MIP } \\
12.90 \\
\\
\text { NIP } \\
7.80\end{array}$ & 1.65 \\
\hline
\end{tabular}

The specificity of a MIP is not the only facet of value when determining a MIPs performance, the selectivity also plays a major role in determining a MIPs potential. Therefore, the selectivity of the MIP 205 was scrutinized by performing binding experiments with analogues of amoxicillin. For this, the aminopenicillins ampicillin and cloxacillin were selected, being similar in chemical structure and in the same class of compounds to that of amoxicillin. The binding experiment was conducted in the same manner as previously outlined, ensuring the concentrations of the aminopenicillin analogues was in 
the same range $(0.1-0.7 \mathrm{mM})$, the mass of $\mathrm{MIP} / \mathrm{NIP}$ utilized $(20 \mathrm{mg}$ ) was constant and the incubation period remained the same. The corresponding binding isotherms were then plotted, and allometrically fitted $\left(y=a x^{b}\right)$ for comparison (Figure 9).

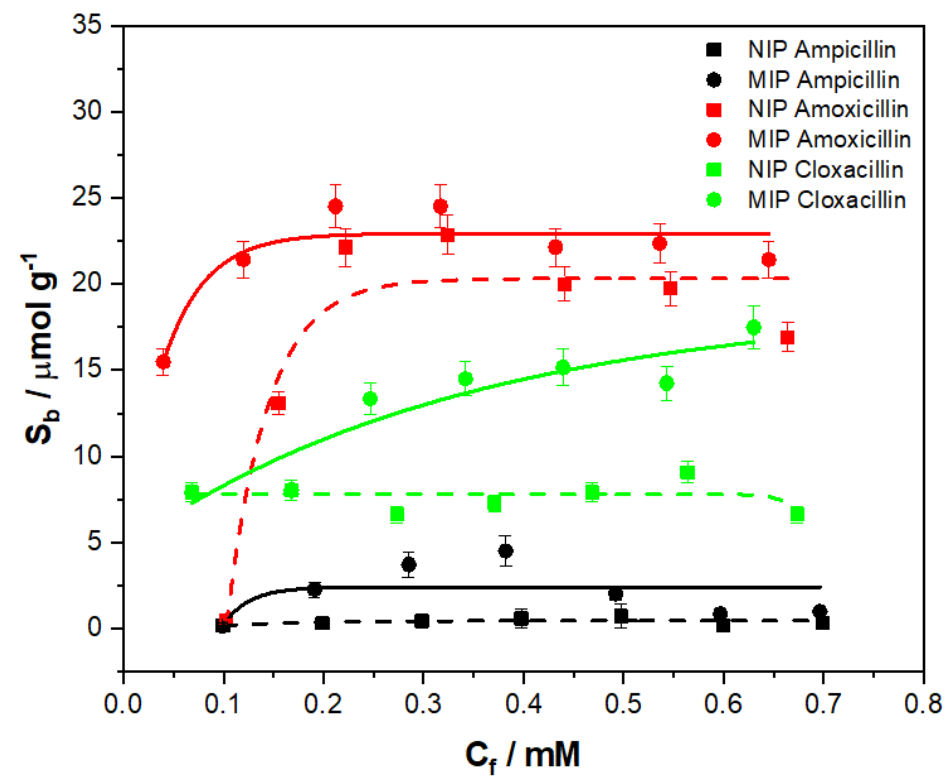

Figure 9. Binding isotherm for the interaction of ampicillin (black squares/circles), cloxacillin (green squares/circles), and amoxicillin (red squares/circles) with MIP 205 where the interactions of both MIP (circles) and NIP (squares) are displayed.

Of the analogues tested cloxacillin proved to have the higher level of interaction in terms of binding capacity with the MIP/NIP 205 out of the two derivative compounds. The interaction with the MIP (green circles) gradually increased with the $C_{f}$, with the allometric fit (green line, $R^{2}=0.81599$ ) describing the data relatively well and yielding a maximum binding capacity (Sb) of $16.43 \mu \mathrm{mol} \mathrm{g}^{-1}$. The interaction of cloxacillin with the NIP (green squares) was also consistent at purveying a binding average $\mathrm{Sb}$ of $7.61 \mu \mathrm{mol} \mathrm{g}{ }^{-1}$. In comparison, the binding for the ampicillin (Figure 9a) was much lower with the MIP (black line, $\mathrm{R}^{2}=0.28487$ ) yielding a maximum $\mathrm{S}_{\mathrm{b}}$ of $4.71 \mu \mathrm{mol} \mathrm{\textrm {g } ^ { - 1 }}$ and the NIP (black dashed line, $R^{2}=0.29573$ ) a maximum of $0.53 \mu \mathrm{mol} \mathrm{g}^{-1}$. When 
comparing the maximum binding capacities of the compounds against that of amoxicillin, it is clear that they are lower. However, further analysis of the selectivity of the MIP can be conducted by calculating IF values for each of the derivatives for a direct comparison of the specific interactions.

As with the monolithic bulk polymerization MIPs the IF was compared at multiple $C_{f}$ values $\left(C_{f}=0.1,0.2\right.$, and $\left.0.3 \mathrm{mM}\right)$, enabling a comparison of the specific binding across a concentration range (Table 3). At the $C_{f}=0.1 \mathrm{mM}$., amoxicillin demonstrates the highest specific interaction with the MIP with ampicillin having the higher level of specific interaction out of the two analogues. This trend however ends abruptly as of $C_{f}=0.2 \mathrm{mM}$ where the amoxicillin specific interaction drops off and becomes similar to that of cloxacillin, while the interaction towards ampicillin increases. As previously stated this is not a huge issue for the sensor as samples tend to contain sub 0.1 $\mathrm{mM}$ concentrations and therefore the interactions at the higher concentrations are less relevant when contextualized.

\section{Selection of dye}

To develop the MIP into a displacement assay capable of producing a visual confirmation for the presence of amoxicillin in solution, different dye molecules must be considered for the application in the assay. Considering the chemical composition of the MIP being used to detect the amoxicillin (methacrylic acid/ethylene glycol dimethacrylate co-polymer) dye molecules that can form ionic and hydrogen bonding interactions with the acidic functionalities present were selected. Malachite green, crystal violet and mordant orange have either amine functionalities (malachite green/ crystal violet) or acidic functionalities (mordant orange) capable of forming these interactions (see Appendix A A1 for chemical structures). The binding interactions of these species with MIP 205 was therefore investigated by means of binding experiments identical to ones discussed prior (Figure 10). The dyes performance was assessed on the maximum binding capacity of the dye compound towards the MIP and the specific interaction (difference between MIP/NIP) exhibited. 

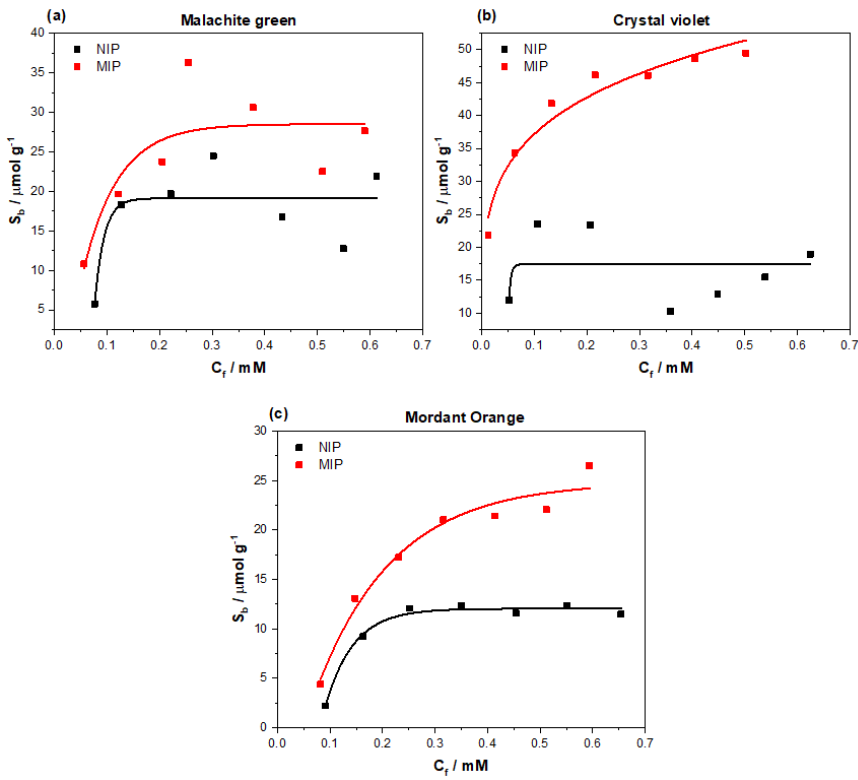

Figure 10. The binding isotherms outlining the interactions of (a) malachite green, (b) crystal violet, and (c) mordant orange with MIP/NIP 205. The plotted values for each of the binding isotherms were extrapolated from the absorbance peaks for each compound at their $\lambda_{\max }$ values $\left(616,590\right.$, and $385 \mathrm{~nm}$ respectively), enabling $C_{f}$ values to be calculated and subsequently $S_{b}$ values.

Of the dyes tested against the MIP, crystal violet was found to hold the highest affinity towards the polymer. The MIP demonstrated a maximum $S_{b}$ of 49.73 $\mu \mathrm{mol} \mathrm{g}{ }^{-1}$ towards crystal violet, being higher than that of affinity towards the amoxicillin template. The same was true for malachite green, with the MIP showing a maximum binding capacity of $28.33 \mu \mathrm{mol} \mathrm{g}^{-1}$, which is once again higher than the observed values for amoxicillin. Mordant orange however was found to have a maximum binding capacity of $24.18 \mu \mathrm{mol} \mathrm{g}^{-1}$ that is closer to that of the template molecule. The specific nature of the interactions to the MIPs was once again calculated by dividing the $\mathrm{Sb}$ of the MIP by that of the NIP at a defined $C_{f}$ (in this case $C_{f}=0.1,0.2$ and $0.3 \mathrm{mM}$ for direct comparison with other values calculated), and the values can be found in Table 4 . 
Table 4. Binding capacities $\left(\mathrm{S}_{\mathrm{b}}\right)$ for malachite green, crystal violet, and mordant orange at $\mathrm{C}_{\mathrm{f}}=0.1 \mathrm{mM}$, and their corresponding imprint factors (IF).

\begin{tabular}{|c|c|c|c|c|c|c|}
\hline Compound & $\begin{array}{c}\mathrm{S}_{\mathrm{b}} / \mu \mathrm{mol} \\
\mathrm{g}^{-1}(\text { at } \mathrm{Cf} \\
=0.1 \\
\mathrm{mM})\end{array}$ & $\begin{array}{l}\text { IF (at } C_{f} \\
=0.1 \\
\mathrm{mM} \text { ) }\end{array}$ & $\begin{array}{c}\mathrm{S}_{\mathrm{b}} / \\
\mu \mathrm{mol} g \\
{ }^{1}\left(\mathrm{at} \mathrm{C}_{\mathrm{f}}=\right. \\
0.2 \\
\mathrm{mM})\end{array}$ & $\begin{array}{c}\text { IF (at } C_{f} \\
=0.2 \\
\mathrm{mM})\end{array}$ & $\begin{array}{c}\mathrm{S}_{\mathrm{b}} / \\
\mu \mathrm{mol} \mathrm{g-} \\
{ }^{1}\left(\text { at } \mathrm{C}_{\mathrm{f}}=\right. \\
0.3 \\
\mathrm{mM})\end{array}$ & $\begin{array}{l}\text { IF (at } C_{f} \\
=0.3 \\
\mathrm{mM})\end{array}$ \\
\hline $\begin{array}{c}\text { Malachite } \\
\text { green }\end{array}$ & $\begin{array}{c}\text { MIP } \\
19.09 \\
\text { NIP } \\
15.22\end{array}$ & 1.25 & $\begin{array}{c}\text { MIP } \\
26.35 \\
\text { NIP } \\
18.89\end{array}$ & 1.39 & $\begin{array}{c}\text { MIP } \\
28.00 \\
\text { NIP } \\
19.03\end{array}$ & 1.47 \\
\hline Crystal Violet & $\begin{array}{l}\text { MIP } \\
36.92 \\
\text { NIP - } \\
\text { MIP }\end{array}$ & - & $\begin{array}{l}\text { MIP } \\
42.69 \\
\text { NIP - } \\
\text { MIP }\end{array}$ & - & $\begin{array}{l}\text { MIP } \\
46.33 \\
\text { NIP - } \\
\text { MIP }\end{array}$ & - \\
\hline $\begin{array}{l}\text { Mordant } \\
\text { Orange }\end{array}$ & $\begin{array}{l}7.31 \\
\text { NIP } \\
3.77\end{array}$ & 1.94 & $\begin{array}{c}15.75 \\
\text { NIP } \\
10.78\end{array}$ & 1.46 & $\begin{array}{c}20.24 \\
\text { NIP } \\
11.85\end{array}$ & 1.71 \\
\hline
\end{tabular}

Due to the nature of the plotted data for crystal violet, there was no reasonable observed line of best fit for the NIP data. The lack of line of best fit therefore made any possible IF calculation for crystal violet invalid and non-comparable to the other dye molecules. Malachite green and mordant orange could however have their IF calculated across the selected range, with mordant orange out performing malachite green at every concentration. The IF value was highest for mordant orange at $C_{f}=0.1 \mathrm{mM}$ demonstrating a 1.94 times greater interaction towards the MIP than NIP. This trend was observed at $\mathrm{C}_{\mathrm{f}}=$ 0.2 and $0.3 \mathrm{mM}$, with the IF varying little from the calculated value at the lowest concentration. Malachite green was calculated to have an increasing IF value across the free concentrations, demonstrating a more specific interaction at higher concentrations compared to low.

When considering the overall binding capacity of each dye and the associated IF values it made most logical sense to select mordant orange to carry forward to the dye displacement assay. The binding capacity of mordant orange reflects a more similar value to that amoxicillin, whereas the overall binding capacity of malachite green is marginally higher so may perform less well in the developed assay (further explanation in discussion section). 


\section{Dye displacement}

The essence of the dye displacement assay relies on the loading of the reporting dye onto the MIP. This was conducted according to the experimental section in accordance to prior literature [26, 27], where a concentrated solution of dye $(1 \mathrm{mM})$ is incubated with the MIP powder $(1 \mathrm{~g})$ for a defined period (30 minutes). After the incubation period is over the now coloured MIP powder is filtered and washed with water until the filtrate has no observable colour and can be confirmed by UV-spectrophotometry. Based on the previous experimentation mordant orange was selected to be preloaded onto the MIP to generate the dye displacement assay.

Once the mordant orange dye was loaded onto the MIPs a dose response was conducted, correlating displaced dye with the concentration of amoxicillin present (Figure 11a). This was achieved by incubating mordant orange-loaded MIP 205 with varying concentrations of amoxicillin $\left(0.000-365.0 \mathrm{mg} \mathrm{L}^{-1}\right)$ ensuring that the volumes added $(5 \mathrm{~mL})$, masses $(40 \mathrm{mg})$ used, and incubation time (1 minute) remained constant throughout the experiment. The analysis was conducted inside glass vials for easy of use, with disposable syringes and filters (pore size $0.45 \mu \mathrm{m}$ ) used to limit potential contamination from external sources. The dose response was conducted between $0.000-365.0 \mathrm{mg} \mathrm{L}^{-1}$ of amoxicillin, in an attempt to determine the minimum concentration require to stimulate the displacement of dye that could be quantified. The spectra clearly shows as the amount of amoxicillin increases in the solution $(\lambda=270 \mathrm{~nm})$, so does the amount of dye displaced into the medium $(\lambda=385 \mathrm{~nm})$, and the lowest value plotted $\left(0.365 \mathrm{mg} \mathrm{L}^{-1}\right)$ still stimulates a quantifiable response. 

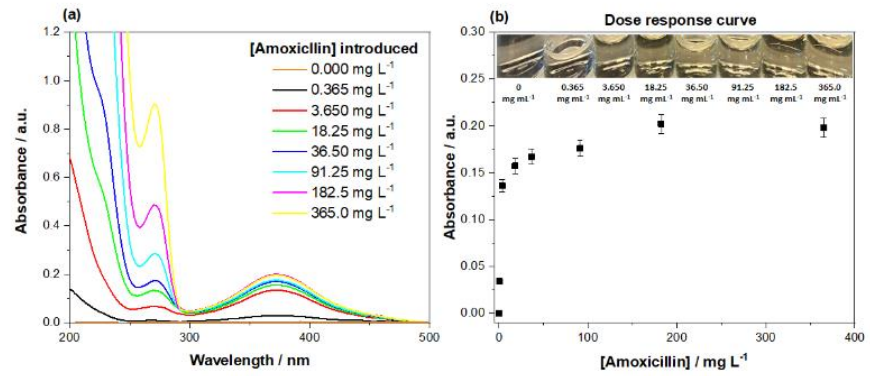

Figure 11. (a) The UV absorbance spectra of the filtrate collected from the incubation of differing concentrations of aqueous amoxicillin with the developed dye loaded MIP, accompanied by (b) the plotted dose response correlating the absorbance $(\lambda=385 \mathrm{~nm})$ of dye released and the concentration of amoxicillin present.

The absorbance of the mordant orange $(\lambda=385 \mathrm{~nm})$ proceeded to be plotted against the concentration of amoxicillin present in the samples (figure 11b), generating the final dose response graph. The data shows a clear trend, gradually plateauing as the concentration of amoxicillin increases. The initial response is quite sensitive, showing a sharp increasing in dye displaced between $0.365 \mathrm{mg} \mathrm{L}^{-1}$ and $3.650 \mathrm{mg} \mathrm{L}^{-1}$. After this the response rapidly diminished, with little to no difference in response to concentrations greater than $91.25 \mathrm{mg} \mathrm{L}^{-1}$. Alongside the collect spectra, photos were taken of the colorimetric response to each concentration and labelled (figure 11b). A clear observable increase in colour is apparent with the initial introduction of the lower concentration, however as the concentrations increase it proved harder to distinguish the concentrations present. This said, there was a clear orange colour to all the samples containing amoxicillin. 

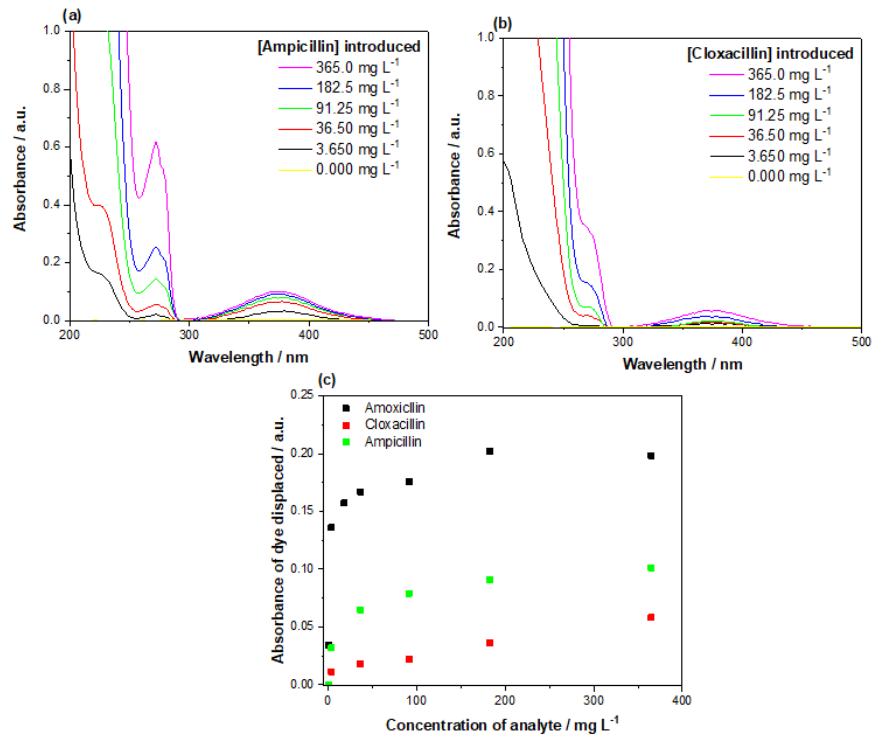

Figure 12. Dose response of the displacement assay towards (a) ampicillin and (b) cloxacillin, with the (c) maximum absorbance of the mordant orange dye $(\lambda=385 \mathrm{~nm})$ plotted against the concentration of analyte present for comparison.

Alongside the response of the assay to amoxicillin, the selectivity of the assay was assessed with increasing concentrations of ampicillin and cloxacillin (Figure 12). Of the two aminopenicillins, ampicillin exhibited a stronger response when incubated with the assay, though the absorbance recorded overall was half that of the amoxicillin. Cloxacillin demonstrated a weaker interaction with the assay; though dye was still displaced, it was at a much lower concentration than what was observed for the other aminopenicillins tested. Overall the reaction of the assay to the amoxicillin is much greater, though the selectivity of the assay clearly extends in a lesser extent towards of compounds of the same class. 


\section{Discussion}

\section{Bulk polymerization MIP analysis}

Of the compositions tested, the methacrylic acid/ ethylene glycol dimethacrylate co-polymer proved to be the most efficient at binding the imprinted amoxicillin. The methacrylic acid has potential to interact with the amine, carboxylic acid, and hydroxyl functionalities within the molecule, offering multiple binding modes between the template and polymer. The acrylamide based MIP 204 did not share the same affinity as its MAA based counterpart, with the trend of the MIP data being poor across the concentration range (hence the data was not fit). The reasoning for this could lie in the fact the carboxylic acid group is too easy to access on the edge of the molecule and therefore the imprints formed do not capture the full chemical architecture. Thus, the difference between MIP and NIP is diminished as the non-specific acrylamide at the surface of the NIP is just as likely to interact as the shallow imprints of the MIP making a trend harder to correlate. MIP 202 replaced the EGDMA with TRIM, yielding a more crosslinked polymeric structure stemming from the increased number of double bonds (3) that TRIM contains compared to that of EGDMA (2). The increased crosslinking provides extract mechanical rigidity to the polymer produced, though this had little impact on performing the overall performance of the MIP. The binding capacity was increased $\left(12.10 \mu \mathrm{mol} \mathrm{g}^{-1}\right)$, but the binding of amoxicillin at low concentrations $\left(\mathrm{C}_{\mathrm{f}}=0.1 \mathrm{mM}\right)$ remained poor compared to that of the NIP. In contrast, MIP 203 had a slightly diminished binding capacity $\left(9.47 \mu \mathrm{mol} \mathrm{g} \mathrm{g}^{-1}\right)$ but performed well at low concentrations when compared to the NIP. This is reflected in the IF values calculated for the MIPs, where it is clear that MIP 203 has the superior specificity towards the amoxicillin over the other MIPs. When analyzing the data at higher $\mathrm{C}_{\mathrm{f}}$ it is apparent that MIP 202 has increase in performance when compared to the NIP, whereas MIP 203 performances begins to slowly diminish. If the IF values are extrapolated and calculated at $\mathrm{C}_{\mathrm{f}}=0.6 \mathrm{mM}$ MIP 202 and 203 have values of 2.08 (NIP: $5.79 \mu \mathrm{mol} \mathrm{g}{ }^{-1}$, MIP: $12.07 \mu \mathrm{mol} \mathrm{g}^{-1}$ ) and 2.01 (NIP: $4.70 \mu \mathrm{mol} \mathrm{g}^{-1}$, MIP: $9.44 \mu \mathrm{mol} \mathrm{g}^{-1}$ ) respectively. The convergence of the IF values indicates that the MIPs actually perform comparably at the higher concentrations and their performance is harder to differentiate. As antibiotics tend to be present at lower concentrations it is logical to select the MIP that performs greater at lower concentrations, and therefore MIP 203 is the composition of choice. 
These results are however surprising when comparing the data to literature, as research found on the development of MIPs towards amoxicillin suggests that acrylamide provides the better binding solution [42]. However, the two acrylamide based MIPs tested in the research outline performed extremely poor when compared to that of the MAA based MIPs. This difference in binding maybe derived from the acidity/basicity of the solution used during the rebinding of the molecule. Amoxicillin is observed to be slightly acidic, offering potential to protonate the amine functionality and provide strong ionic interactions with the MAA in the developed polymers [43]. By altering the solution to be more basic nature the acidic functionalities in the molecule could be stripped of their protons, facilitating ionic bond formation between the carboxylic functionalities and acrylamide in a MIP.

The general particle morphology for the bulk imprinted MIPs proved to be very heterogeneous, though this is to be expected as bulk imprinting involves the generation of polymeric monolith that is then ground into a fine powder [44]. The large discrepancy in particle shape and size yields poor performing MIPs, reducing MIP reproducibility in intra and inter sample analysis as particles sizes differ so vastly.

\section{Emulsion MIP structure}

Emulsion polymerization was utilized in an attempt to increase the homogeneity in observed particle sizes and morphologies. As emulsion polymerization combines two phases to form an emulsion between an organic and aqueous medium, it is an ideal tool to produce more uniform particles [45]. In theory, there is little difference between monolithic bulk polymerization and emulsion polymerization, bar the use of water and a surfactant to generate small microspheres in comparison to a monolithic polymer [46]. The incorporation of a surfactant (SDS) helps stabilize the formed emulsion, producing micelles containing the lipophilic components (monomer, crosslinker, initiator) of the reaction and therefore acts as a small reactor that enables localized polymerization without it propagating throughout the whole solution [45]. Literature does however show that the surfactant and presence of water can have negative effects on the binding capabilities of the MIP [47], though there is opposing literature that suggests the presence of water may in fact help the formation and function of the MIP [48]. It is to be noted that different interactions occur during each process, and the purpose of the research is to see how generating more homogenous smaller particles by 
emulsion polymerization compare to a crushed monolith of the same composition. This said the size of the micelles formed still depends on how well mixed the organic and aqueous phases are, therefore an investigation into how the stirring affected the achieved particle sizes was conducted. Another consideration lies in the fact that the monolithic bulk composition was directly transferred into an emulsion type setting, which means that DMSO was still used in the organic phase as a porogen. DMSO is miscible with water that could prove problematic for the formation of micelles, though the other components present are a less soluble and still enable micelle formation. There has been research conducted on systems that used DMSO as an alternative solvent for micelle formation when aqueous conditions would not be feasible [49]. It has been shown that micelles still form due to the high polarity of DMSO and a surfactant can still function to form micelles at the phase boundaries between polar and non-polar interfaces [50]. In the case of the generation of MIPs in this research, it could be speculated that DMSO would distribute between the water and less soluble components of the mixture, making it present inside and outside the micelle formation. However, a further study would have to be conducted to thoroughly investigate the true mechanism in this circumstance.

An overhead stirrer was chosen for the reaction process as it provided greater control over the speed the mixture was subjected to, and ensured that the whole mixture received the same level of stirring compared to that of a magnetic stirrer. Ensuring different rpm's could successfully be applied across the solution a range of $600-1400 \mathrm{rpm}$ was selected. Agitation at the lower speeds yielded larger particles $(50-100 \mu \mathrm{m})$ with higher standard deviations, whereas higher speeds produced small particles $(5-30 \mu \mathrm{m})$ with a smaller deviation. When plotting the particle size against rpm a clear relation is viewed, with the particle sizes achieved plateauing above $1000 \mathrm{rpm}$. Though the particle sizes differs with rpm, the particle morphology does not. Optical microscope images reveal that the spherical morphologies of the particles are consistent across the speeds tested. Thus, the emulsion polymerization demonstrates a visible increase in the homogeneity of particle sizes and structure when compared to that of the bulk polymerization method. 


\section{Binding of Emulsion MIP}

When analyzing the emulsion MIP 205 data it is clear that it possess a higher binding capacity $\left(22.53 \mu \mathrm{mol} \mathrm{\textrm {g } ^ { - 1 }}\right)$ towards amoxicillin when compared to the monolithic bulk generated particles $\left(9.89 \mu \mathrm{mol} \mathrm{g}^{-1}\right)$. The increased homogeneity of the particles in MIP 205 are the clear source of this increased capacity, providing smaller, more uniform particle sizes that facilitate greater mass transfer and surface area when compared to the roughly ground particles [51]. MIP 205 performs greatly at lower concentrations (below $C_{f}=0.15 \mathrm{mM}$ ) and quickly saturates, whereas MIP 203 performs across the full concentration range. The IF values compared in table 3 relates the performance of the MIPs at different free concentrations $\left(\mathrm{C}_{\mathrm{f}}=0.1,0.2\right.$, and $\left.0.3 \mathrm{mM}\right)$, analyzing the specific interaction of the MIPs more clearly. These IF values compliment figure 8, giving a metric of the decreased performance of MIP 205 past $C_{f}=0.15 \mathrm{mM}$. It can be speculated that the loss in performance at higher concentrations is a direct result of simply transferring monolithic bulk optimized components to the emulsion methodology, or as previously mentioned the presence of water and surfactant during polymerization reducing the binding affinities at the higher concentrations. However, this drop in performance at higher concentrations is inconsequential as the concentration range for antibiotics within wastewater, effluent, and/or milk are much lower (286.7 - $\left.909 \mathrm{ng} \mathrm{L}^{-1}\right)$ in concentration $[52,53]$.

Exposure of the MIP 205 to other antibiotic analogues (ampicillin and cloxacillin) reveal that the MIP performances well at binding cloxacillin, whereas it poorly binds ampicillin. Between the two analogues tested ampicillin holds the closer resemblance to amoxicillin, though the structure lacks the presence of the hydroxyl functionality of the terminal aromatic system. The absence of this group seems to critically affect the binding of ampicillin, with a diminished maximum binding capacity of $4.71 \mu \mathrm{mol} \mathrm{g} \mathrm{g}^{-1}$ observed. Loss of the strong hydrogen bond acceptor/donor functionality seems the rational for this, being in an easily accessible sterically unhindered position [54]. Cloxacillin does not display the same reduced binding capacity $\left(16.43 \mu \mathrm{mol} \mathrm{g}{ }^{-1}\right)$, despite its much larger molecular weight and bulk chemical composition. The structure does however retain the presence of the oxygen heteroatom alongside an additional nitrogen, which could be the argument for this. The addition of the nitrogen and subsequent lone pair of electrons would facilitate extra binding via a hydrogen donor interaction, and even an ionic 
interaction as the functionality becomes positively charged by accepting a proton [54].

The specific interaction (IF) of each analogue was once again evaluated at $\mathrm{C}_{\mathrm{f}}=$ $0.1,0.2$ and $0.3 \mathrm{mM}$ to enable a direct comparison of each of the compounds (Table 3). When analyzing these values at $\mathrm{C}_{\mathrm{f}}=0.1 \mathrm{mM}$ it is clear that amoxicillin has the highest IF value by quite some way, though now it is observed that ampicillin actually has the higher specific interaction when compared against cloxacillin. This maybe down the aforementioned reasons with ampicillin being closer to the structure of amoxicillin so therefore can bind more specifically, whereas cloxacillin has more groups that can provide non-specific interactions. As the IF is calculated at higher concentrations $\left(C_{f}=0.2\right.$ and 0.3 $\mathrm{mM}$ ) ampicillin interacts more specifically with the MIP, whereas cloxacillin and amoxicillin interact with the MIP in a more comparable and similar manner. Though the intended sensor is developed towards amoxicillin, there is an argument to be made that a broader sensor for aminopenicillins as a whole is more preferable as it would facilitate broad spectrum testing of samples. Therefore, the indicated affinity of the sensor towards cloxacillin could be of benefit depending on the setting the sensor is deployed in.

\section{Selection of a dye for displacement}

Malachite green and crystal violet were selected for testing as potential dyes to be displaced based on their previous use in other research [26, 27]. Their chemical structures contained terminal amine functionalities that offer ionic bonding to the methacrylic acidic functionalities in the MIP. The observed binding capacity of malachite green and crystal violet reflect this, with binding capacity surpassing that of amoxicillin $\left(28.33 \mu \mathrm{mol} \mathrm{g}^{-1}\right.$ and $\left.49.73 \mu \mathrm{mol} \mathrm{g}-1\right)$. The increased interactions between the dyes and MIP can stem from the increased number of easily accessible amine functionalities present, and/or the relative size of the molecules when compared to that of amoxicillin [55]. Mordant orange on the other hand contains an azo functionality placed between two aromatic systems, making it more hindered and harder to interact with. This fact is reflected in the maximum binding capacity observed $(24.18 \mu \mathrm{mol} \mathrm{g}-1)$, though mordant orange possess a nitro and carboxylic acid functional that can provide additional interactions with the MIP.

Previous research has demonstrated that there is a relationship between the imprint factor of the dye being displaced and the imprint factor of the target 
molecule displacing said dye [26, 27]. For dye displacement to be successful the IF value of the dye must be lower than that of the template molecule at the defined free concentration. Both malachite green and mordant orange have a lower IF value than amoxicillin at the lower free concentrations, making them both potentially suitable as dyes in the displacement assay. However, the prior research also suggests that the dye molecule must have a somewhat similar binding capacity to the MIP as the template molecule; otherwise, the dye would be less easily displaced. Out of these two dyes, mordant orange has the binding capacity more similar to that of amoxicillin, whereas malachite green is observed to have a higher maximum binding capacity that would not bode well for the displacement mechanism. Therefore, of the two molecules, mordant orange is the clear choice for the displacement assay.

\section{Dye displacement assay}

The dose response reviled that an observable displacement of dye was apart when the assay was incubated with $0.365 \mathrm{mg} \mathrm{L}^{-1}$, being clear on the UV-spectra and by analysis with the naked eye. After this point the inspection of the colour of the filtrate proved more difficult as the colour gradient between samples is not always obviously. This said, a clear distinction between absorbance is seen in the UV-spectra with the amount of dye displaced correlating to the amount of amoxicillin present. Normally other organic pollutants are expected to present that could mask/interfere (absorb at a same or similar wavelength) with the amoxicillin absorbance peak; therefore, it is beneficial having the higher wavelength dye present that is less likely to experience interferences/masking [56]. Past $91.25 \mathrm{mg} \mathrm{L}^{-1}$ however there is little change in the amount of dye displaced, meaning higher concentrations of amoxicillin could not be correlated with a specific absorbance of dye. These results lend into the assay being used in a semi-quantitative manner, enabling the user to visually differentiate between low and high concentrations. The linear range unfortunately is lacking, which would impede the sensor in an environment where quantitation of the presence of amoxicillin is necessary.

Selectivity tests on the assay revealed that the assay is also sensitivity towards the presence of ampicillin and cloxacillin. Figure 12a shows that ampicillin elicits a higher amount of dye displacement when compared to cloxacillin, though this amount is still only half the amount that the amoxicillin achieves. In the context of waste analysis for antibiotics it would be fruitful to have an assay that is capable of detecting a wider range of antibiotics compared to just 
a singular structure. The selectivity of the assay towards other aminopenicillins can therefore be seen as a positive, opening the sensor to the possibility of broad range analysis across the class of compounds. As previously discussed, it is logical that other compounds of the same class interact with the assay as they did with the MIP. If the assay was to be developed further towards the sole purpose of detecting amoxicillin, then the selectivity of the MIP must therefore be improved to remove the cross selectivity observed in the assay. This may however prove troublesome, aminopenicillins share very similar chemical architectures, making it more sensible to expand the capabilities of the MIP towards class determination rather than a compound specific analysis. 


\section{Conclusions}

Overall, the research demonstrates how a colorimetric assay for the detection of amoxicillin can be easily generated in just a few steps. The work utilizes simple bulk MIPs to discover polymeric compositions that interact with amoxicillin and builds these findings into a more refined MIP morphology that improves sensitivity and homogeneity by means of emulsion polymerization. During the synthesis of these more refined particles, a relationship between the speed of agitation and particles sizes produced was observed. This relationship could be exploited to make more reliable MIP sizes on a larger scale that holds both academic and industrial value. These more homogeneous particles demonstrated high specificity towards amoxicillin at low concentrations $\left(\mathrm{C}_{\mathrm{f}}=\right.$ $0.1 \mathrm{mM}$ ), while proving selective and showing minimal interaction with compounds of the same class (ampicillin and cloxacillin). Alongside aminopenicillin analogues, various dyes (malachite green, crystal violet, and mordant orange) were tested for their specific binding towards the MIP and their interactions studied. Malachite green and crystal violet have been previously used in literature to produce dye displacement assays, though mordant orange has never been tested for such a possibility. This research therefore pushes the boundaries that are currently in place for the selection of dyes for these kind of MIP-based dye displacement assays. Once the optimum dye was identified, the developed MIP was easily converted into a visual sensor by the simple loading of mordant orange that could easily be displaced in the presence of the template molecule - amoxicillin.

Overall, the novelty of the research lies in the fact that this is the first time a MIP-based dye displacement assay has been generated for amoxicillin using this format with the combination of mordant orange. The findings from this research has potential to be used in both a quantitative and semi-quantitative manner depending on if analytical apparatus is on hand. The key technical advantage being shorter analysis time, no sample pre-treatment and portability. In short, the methods outlined in this paper could be utilized in the rapid analysis of aqueous samples for the unwanted presence of the antibiotic amoxicillin. 


\section{Appendix A}

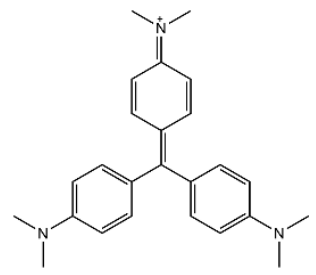

(a) Crystal Viloet

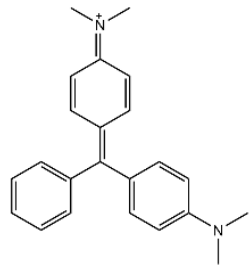

(b) Malachite green<smiles>O=[N+]([O-])c1ccc(N=Nc2ccc(O)c(O)c2)cc1</smiles>

(c) Mordant orange

Figure A1. The chemical structure of (a) Crystal violet, (b) Malachite green, and (c) Mordant orange. 


\section{Chapter 6 References}

[1] Kummerer, K. Significance of antibiotics in the environment. J Antimicrobial Chemo. 2003, 52, 5-7.

[2] Li, Y.; Liu, B.; Zhang, X.; Wang, J.; Gao, S. The distribution of veterinary antibiotics in the river system in a livestock-producing region and interactions between different phases. Environ Sci Pollut Res. 2016, 23, 16542-16551.

[3] Zhi, S.; Shen, S.; Zhou, J.; Ding, G.; Zhang, K. Systematic analysis of occurence, density and ecological risks of 45 veterinary antibiotics: Focused on family livestock farms in Erthai Lake basin, Yunnan, China. Environ Pollut. 2020, 267, 115539.

[4] Wang, L.; Yang, H.; Zhang, C.; Mo, Y.; Lu, X. Determination of oxytetracycline, tetracycline and chloramphenicol antibiotics in animal feeds using subcritical water extraction and high performance liquid chromatography. Anal Chimi Acta. 2008, 619(1), 54-58.

[5] Daghrir, R.; Drogui, P. Tetracycline antibiotics in the environment: a review. Environ Chem Letters. 2013, 11, 209-227.

[6] Michael, I.; Rizzo, L.; McArdell, C. S.; Manaia, C. M.; Merlin, C.; Schwartz, T.; Dagot, C.; Fatta-Kassinos, D. Urban wastewater treatment plants as hotspots for the release of antibiotics in the environment: a review. Water Research. 2013, 47(3), 957-995.

[7] Kantiani, L.; Farre, M.; Barcelo, D.; Barcelo, D. Analytical methodologies for the detection of $\beta$-lactam antibiotics in milk and feed samples. TrAC. 2009, 28(6), 729-744.

[8] Jing, T.; Wang, Y.; Dai, Q.; Xia, H.; Niu, J.; Hao, Q.; Mei, S.; Zhou, Y. Preperation of mixed-templates molecularly imprinted polymers and investigation of the recognition ability for tetracycline antibiotics. Biosens \& Bioelec. 2010, 25(10), 2218-2224.

[9] Luo, Q.; Yu, N.; Shi, C.; Wang, X.; Wu, J. Surface plasmon resonance sensor for antibiotics detection based on photo-initiated polymerization molecularly imprinted array. Talanta. 2016, 161, 797-803.

[10] Naklua, W.; Suedee, R.; Lieberzeit, P. A. Dopaminergic receptor-ligand binding assay based on molecularly imprinted polymers on quartz crystal microbalance sensors. Biosensors \& Bioelectronics. 2016, 81, 117-124.

[11] Liu, M.; Tran, T. M.; Elhaj, A. A. A.; Torsetnes, S. B.; Jensen, O. N.; Sellergren, B.; Irgum, K. Molecularly Imprinted Porous Monolithic Materials From MelamineFormaldehyde for Selective Trapping of Phosphopeptides. Anal. Chem. 2017, 89, 94919501.

[12] Lowdon, J. W.; Alkirkit, S. M. O.; Mewis, R. E.; Fulton, D.; Banks, C. E.; Sutcliffe, O. B.; Peeters, M. Engineering molecularly imprinted polymers (MIPs) for the selective extraction and quantification of the novel psychoactive substance (NPS) methoxphenidine and its regioisomers. Analyst. 2018, 143, 2081-2089.

[13] Tian, J.; Bai, J.; Peng, Y.; Qie, Z. ; Zhao, Y. ; Ning, B. ; Xiao, D. ; Gao, Z. A core-shellstructured molecularly imprinted polymer on upconverting nanoparticles for selective and sensitive fluorescence sensing sulfamethazine. Analyst. 2015, 140, 5301-5307.

[14] Qu, Y.; Qin, L.; Lui, X.; Yang, Y. Reasonable design and sifting of microporous carbon nanosphere-based surface molecularly imprinted polymer for selective removal of phenol from wastewater. Chemosphere. 2020, 251, 126376. 
[15] Pisarev, O. A.; Polyakova. I. V. Molecularly imprinted polymers based on methacrylic acid and ethyleneglycol dimethacrylate for L-lysine recognition. Reactive $\mathcal{E}$ Functional Polymers. 2018, 130, 98-110.

[16] Cantarella, M.; Carroccio, S. C.; Dattilo, S.; Avolio, R.; Castaldo, R.; Puglisi, C.; Privitera, V. Molecularly imprinted polymer for the selective absorption of diclofenac from contaminated water. Chem Eng J. 2019, 367, 180-188.

[17] Vandenryt, T.; van Grinsven, B.; Eersels, K.; Cornelis, P.; Kholwadia, S.; Cleij, T.J.; Thoelen, R.; De Ceuninck, W.; Wagner, P. Single-shot detection of neurotransmitters in whole-blood samples by means of the heat-transfer method in combination with synthetic receptors. Sensors, 2017, 17, 2701.

[18] Eersels, K.; Dilien, H.; Lowdon, J. W.; Redeker, E. S.; Rogosic, R.; Heidt, B.; Peeters, M.; Cornelis, P.; Lux, P.; Reutelingsperger, C. P.; Schurgers, L. J.; Cleij, T. J.; \& Grinsven, B. A novel biomimetic tool for assessing vitamin $\mathrm{K}$ status based on molecularly imprinted polymers. Nutrients. 2018, 10, 751.

[19] Casadio, S.; Lowdon, J. W.; Betlem, K.; Ueta, J. T.; Foster, C. W.; Cleij, T. J.; Grinsven, B.; Sutcliffe, O. B.; Banks, C. E.; Peeters, M. Development of a novel flexible polymer-based biosensor for the thermal detection of noradrenaline in aqueous solutions. Chem Eng J. 2017, 315, 459-468.

[20] Lowdon, J. W.; Dilien, H.; Singla, P.; Peeters, M.; Cleij, T. J.; van Grinsven, B.; Eersels, K. MIPs for commercial application in low-cost sensors and assays - An overview of the current status quo. Sensors \& Actuators B: Chem. 2020, 325, 128973.

[21] McNiven, S.; Kato, M.; Levi, R.; Yano, K.; \& Karube, I. Chloramphenicol sensor based on an in situ imprinted polymer. Analytica Chimica Acta. 1998, 365, 69-74.

[22] Greene, N. T.; \& Shimizu, K. D. Colorimetric molecularly imprinted polymer sensor array using dye displacement. J. Am. Chem. Soc. 2005, 127, 5695-5700.

[23] Mattsson, L.; Xu, J.; Preininger, C.; Tse Sum Bui, B.; \& Haupt, K. Competitive fluorescent pseudo-immunoassay exploiting molecularly imprinted polymers for the detection of biogenic amines in fish matrix. Talanta. 2018, 181, 190-196.

[24] Li, C.; Ngai, M. H.; Reddy, K. K.; Leong, S. C. Y.; Tong, Y. W.; \& Chai, C. L. L. A fluorescence-displacement assay using molecularly imprinted polymers for the visual, rapid, and sensitive detection of the algal metabolites, geosim and 2-methylisoborneol. Analytica Chimica Acta. 2019, 1066, 121-130.

[25] Silverio, O. V.; So, R. C.; Elnar, K. J. S.; Malapit, C. A.; \& Nepomuceno, M. C. M. Development of dieldrin, endosulfan, and hexachlorobenzene-imprinted polymers for dye-displacement array sensing. J. Appl. Polym. Sci. 2017, 134(2), 44401.

[26] Lowdon, J. W.; Eersels, K.; Rogosic, R.; Heidt, B.; Diliën, H.; Redeker, E. S.; Peeters, M.; van Grinsiven, B.; Cleij, T. J. Substrate Displacement Colorimetry for the Detection of Diarylethylamines. Sens. Actuators B Chem. 2019, 282, 137-144.

[27] Lowdon, J. W.; Eersels, K.; Arreguin-Campos, R.; Caldara, M.; Heidt, B.; Rogosic, R.; Jimenez-Monroy, K. L.; Cleij, T. J.; Diliën, H.; van Grinsven, B. A molecularly imprinted polymer-based dye displacement assay for the rapid visual detection of amphetamine in urine. Molecules. 2020, 25, 5222. 
[28] Romano, A.; Valluzzi, R. L.; Caruso, C.; Zaffiro, A.; Quaratino, D.; Gaeta, F. Tolerability of cefazolin and ceftibuten in patients with IgE-mediated aminopenicillin allergy. J Allergy Clinical Immu: In Practice. 2020, 8(6), 1989-1993.

[29] Mollmann, U.; Heinisch, L.; Bauernfeind, A.; Kohler, T.; Ankel-Fuchs, D. Siderophores as drug delivery agents: application of the "Trojan Horse" strategy. BioMetals. 2009, 22, 615-624.

[30] Kushwaha, A.; Srivastava, J.; Singh, A. K.; Anand, R.; Raghuwanshi, R.; Rai, T.; Singh, M. Epitope imprinting of mycobacterium leprae bacteria via molecularly imprinted nanoparticles using multiple monomers approach. Biosens \& Bioelec. 2019. 145, 111698.

[31] Duan, F.; Chen, C.; Zhao, X.; Yang, Y.; Liu, X.; Qin, Y. Water-compatible surface molecularly imprinted polymers with synergy of bi-functional monomers for enhanced selective adsorption of bisphenol A from aqueous solution. Environ. Sci.: Nano. 2016, 3, 213-222.

[32] Wloch, M.; Datta, J. Chapter Two - Synthesis and polymerization techniques of molecularly imprinted polymers. Compre. Anal. Chem. 2019, 86, 17-40.

[33] Erturk, G.; Mattiasson, B. Molecular imprinting techniques used for the preparation of biosensors. Sensors. 2017, 17(2), 288.

[34] Villa, C. C.; Sanchez, L.; Valencia, G. A. ; Ahmed, S. ; Gutierrez, T. J. Molecularly imprinted polymers for food applications: A review. Trends in food Sci Tech. 2021, 111, 642669.

[35] Ou, H.; Chen, Q.; Pan, J.; Zhang, Y.; Huang, Y.; Qi, X. Selective removal of erythromycin by magnetic imprinted polymers synthesized from chitosan-stabilized pickering emulsion. J Hazardous Mat. 2015, 289, 28-37.

[36] Pan, J.; Li, L.; Hang, H.; Wu, R.; Dai, X.; Shi, W.; Yan, Y. Fabrication and evaluation of magnetic/hollow double-shelled imprinted sorbents formed by pickering emulsion polymerization. Langmuir. 2013, 29(25), 8170-8178.

[37] Lim, K. F.; Holdsworth, C. I. Effect of formulation on the binding efficiency and selectivity of precipitation molecularly imprinted polymers. Molecules. 2018, 23(11), 2996.

[38] Pardeshi, S.; Singh, S. K. Precipitation polymerization: a versitile tool for preparing molecularly imprinted polymer beads for chromatography applications. RSC Adv. 2016, 6, 23525-23536.

[39] Canfarotta, F.; Poma, A.; Guerreiro, A.; Piletsky, S. Solid-phase synthesis of molecularly imprinted nanoparticles. Nature Proto. 2016, 11, 443-455.

[40] Refaat, D.; Aggour, M. G.; Farghali, A. A. ; Mahajan, R. ; Wiklander, J. G.; Nicholls, I. A.; Piletsky, S. A. Strategies for molecular imprinting and the evolution of MIP nanoparticles as plastic antibodies - synthesis and applications. Int. J. Mol. Sci. 2019, 20(24), 6304.

[41] Orowitz, T. E.; Sombo, P. P. A. A. A.; Rahayu, D.; Hasanah, A. N. Microsphere polymers in molecular imprinting: Current and future perspectives. Molecules. 2020, 25(14), 3256.

[42] Jamieson, O.; Soares, T. C. C.; Faria, B. A. Hudson, A.; Mecozzi, F.; Rowley-Neale, S. .J; Banks, C. E.; Gruber, J.; Novabovic, K.; Peeters, M.; Crapnell, R. D. Screen printed electrode based detection systems for the antibiotic amoxicillin in aqueous samples utilizing molecularly imprinted polymers as synthetic receptors. Chemosensors. 2020, 8(5). 
[43] Rolinson, G. N. Laboratory evaluation of amoxicillin. J Intect Diseases. 1974, 129, S139S145.

[44] Katz, A.; Davis, M. E. Molecular imprinting of bulk, microporous silica. Nature. 2000, 403, 286-289.

[45] Alabdullah, S. S. M.; Ismail, H. K.; Ryder, K. S.; Abbott, A. P. Evidence supporting an emulsion polymerization mechanism for the formation of polyaniline. Electochimica Acta. 2020, 354, 136737.

[46] Wang, D.; Zhu, J.; Yao, Q.; Wilkie, C. A. A comparison of various methods for the preparation of polystyrene and poly(methyl methacrylate) clay nanocomposites. Chem. Mater. 2002, 14(9), 3837-3843.

[47] Silva, R. C. S.; Santos, M. N.; Pires, B. C.; Dinali, L. A. F.; Suquila, F. A. C.; Tarley, C. R. T.; Borges, K. B. Assessment of surfactants on performance of molecularly imprinted polymer toward absorption of pharmaceutical. J. Envi. Chem. Eng. 2019, 7(2). 103037.

[48] Chen, L.; Wang, X.; Lu, W.; Wu, X.; Li, J. Molecular imprinting: perspectives and applications. Chem. Soc. Rev. 2016, 45, 2137-2211.

[49] Crespy, D.; Landfester, K. Synthesis of polyvinylpyrrolidone silver nanoparticles hybrid latex in non-aqueous miniemulsion at high temperature. Polymer. 2009, 50, 16161620.

[50] Crespy, D.; Landfester, K. Making dry fertile: A practical tour of non-aqueous emulsions and miniemulsions, their preparation and some applications. Soft Matter. 2011, 7, 11054-11064.

[51] Kim, H.; Kaczmarski, K.; Guiochon, G. Mass transfer kinetics on the heterogeneous binding sites of molecularly imprinted polymers. Chem Eng Sci. 2005, 60(20), 5425-5444.

[52] Coutu, S.; Wyrsch, V.; Wynn, H. K.; Rossi, L.; Barry, D. A. Temporal dynamics of antibiotics in wastewater treatment plant influent. Sci Total Environ. 2013, 458-460, 20-26.

[53] Li, J.; Cheng, W.; Xu, L. ; Strong, P. J. ; Chen, H. Antibiotic-resistant genes and antibiotic-resistant bacteria in the effluent of urban residential areas, hospitals, and municipal wastewater treatment plant system. Environ Sci Pollu Res. 2015, 22, 4587-4596.

[54] Zhang, T.; Lui, F.; Chen, W.; Wang, J.; Li, K. Influence of intramolecular hydrogen bond of templates on molecular recognition of molecularly imprinted polymers. Anal Chimica Acta. 2001, 450, 53-61.

[55] Andersson, L. I. Molecular imprinting: developments and application in the analytical chemistry field. J Chrom B: Biomed Sci Appl. 2000, 745, 3-13.

[56] Soovali, L.; Room, E.; Kutt, A.; Kaljurand, I.; Leito, I. Uncertainty sources in UV-vis spectrophotometric measurement. Accreditation and quality assurance. 2006, 11, $246-255$. 


\section{Chapter 7}

General Discussion

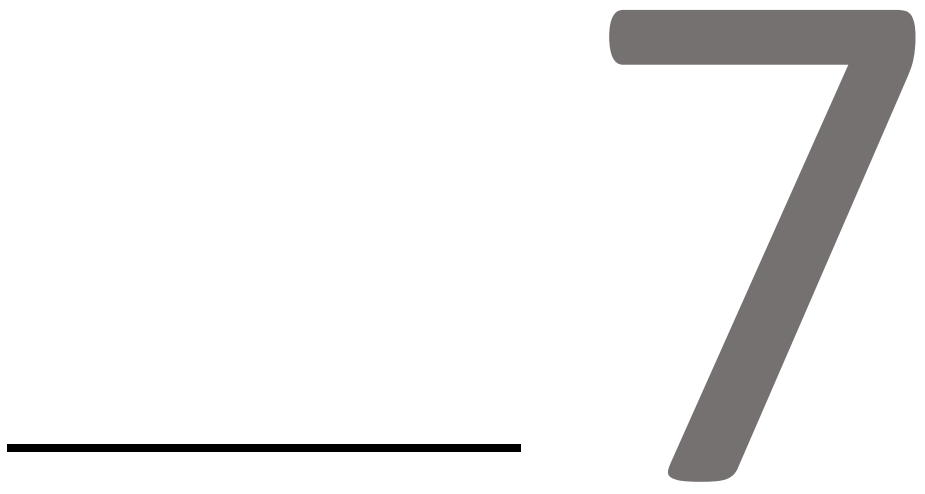




\section{General Discussion}

The research given in this thesis has highlighted how MIPs are a powerful tool in the field of chemical sensing. It has been demonstrated how MIPs can be grown and grafted directly to the surface of substrates (Chapter 3), used as receptor layer in a classical biomimetic sensor concept or transformed into a dye displacement assay for visual verification of new psychoactive substances (Chapter 4), amphetamine (Chapter 5), and aminopenicillins (Chapter 6).

\section{Surface grafting of polymeric receptor layers}

The direct grafting of the polymeric receptors onto the surface of aluminium substrates overcame issues related to the deposition of MIP particles for the use as receptor layers. The reported data showed an increased sensitivity versus the more traditionally deposited polymeric receptors, increasing the LoD of the setup by an order of magnitude. Introducing a methodology that could easily achieve a $15 \mathrm{nM}$ LoD in comparison to $150 \mathrm{nM}$, making the analytical technique more applicable with potential real world analyte concentrations [1]. The thickness of the polymeric layers produced was found to be proportional to the incubation time with the polymeric solution, with an optimum layer thickness of $110 \mu \mathrm{m}$ based on the constant A, representing the fitting of each thickness dose response curve. Compared to current coating film sizes the layers grafted are extremely thick, though the data demonstrated that this had some benefit (e.g. the optimum value for sensitivity being higher than the lower thickness values, and a thick deposited layer lends itself to being more robust).

The grafted polymeric receptors were tailored towards the detection of 2methoxphenidine, targeting a class of compounds that falls under the branch of New Psychoactive Substances (NPS). Compared to more common drugs of abuse, NPS have few tailor made methods for their detection, though it is becoming more common [2]. Current forms of analysis use GC-MS, LC-MS and even MS-MS for their detection, though for the identification of these compounds the operator has to have some rough indication of the type of compound being handled so the correct methodology associated with these techniques can be selected [3]. This means there is currently no scenario where one method encompasses wide spectrum testing to offer analysis across the NPS field. This means that NPS compounds pass by undetected, until they are isolated and a methodology developed for their detection [4]. 
The technology presented in this segment could be offered as an alternative to the current methods. In the current form the receptor maybe lacking the ability to detect multiple analytes, though a generic receptor for different classes of NPS could be generated allowing the wide-spectrum analysis. In terms of LoD and sample analysis, a large proportion of drugs analysis is done on seized samples where sample size (mass) is not an issue [5]. This means an ultralow limit of detection is not essential for analysis, and the ability of the platform to confirm the presence of a target compound is the main goal. The methodology associated with sample analysis remains constant in the thermal method proposed, with the receptors for each analysis being the only element that differs. Therefore, it can be seen with further development of MIPs for NPS, a potential generic method of confirming their presence could be developed and hold great value against the current methods.

There are no specific drawbacks to the outlined technology, though things can always be improved. The development of the receptor layers could be tackled from a different viewpoint, functionalizing the surface of the substrate and chemically binding nanoMIPs to the surface opposed to the thick grafted layers reported [6]. This could potentially improve the LoD of the setup further, though it would be seen if this has any impact on the selectivity of the approach. Improvements to the flow cell design and the thermal couples used in the analysis of the thermal resistance may yield more reliable and reproducible readings, and could be used to further the sensitivity of the method [7]. The culmination of these proposed changes could drastically improve a research line that arguable already has a lot of potential impact.

\section{Substrate Displacement Colorimetry (SDC): Dye displacement assays}

The use of rapid colorimetric assays for the detection of biological and chemical markers has been an expanding field for the last half a century. Their popularity lies in their ease of use and low price point, with the time efficient manner which they convey results adding desirability [8]. MIPs commonly are associated with electrical readout technologies, with few assay format MIP systems presented as of to date. The few assays that currently exist are still classified as emerging technologies, with researchers and companies experimenting with what can be achieved [9]. 
In chapter 4, 5, \& 6 the idea of a simple MIP-based dye displacement assay was brought forward, investigating the relationship between MIP-dye binding and determining what factors affect the displaceability of the dye when a target analyte is introduced to the system. The idea of assigning a binding factor that in essence is a reflection of the commonly used imprinting factor was introduced, enabling a metric to be correlated with how well a molecule interacts with the MIP in comparison to a NIP of the same composition. The metric was correlated with the imprint factor (IF) of various MIP compositions towards the target analyte, predicting where the dye would be displaced in the presence of the chosen analyte. A freehold value was discovered, meaning the IF of the MIP the template molecule had to be greater than the binding factor of the prebound dye for displacement to occur. Thus, a basic concept of a mechanism behind dye displaced was formulated, and built upon for the development of the dye displacement assays.

The initial paper (chapter 4) analyzed the candidacy of multiple dye molecules, assessing their capabilities to bind to the developed MIP. Their affinity was quantisied as a binding factor that was directly compared to the native imprinting factor of the MIP. Thus ensuring the selected molecule had a greater enough difference between these two values to elicit a displacement reaction. Other factors concerning the extinction coefficient of each dye was taken into consideration when finally selecting malachite green for displacement assay. Malachite green exhibited the greatest difference between its own binding factor and the imprinting factor of the MIP, while having a high extinction coefficient. The high extinction coefficient is beneficial in this form of assay as in enables lower concentrations of dye to be detected by means of UV-vis due to intense chromophore present within the molecule, yielding an intense quantifiable band in the absorbance spectra [10]. Malachite green is just one of hundreds of dyes that have this property, screening them all would be impractical (time consuming) but it would be logical that there is a more suitable dye to be identified for future development of the assay. Another consideration could be the use of dye-analyte conjugates, providing a labelled version of the target that could be displaced [11]. The possible downsides to this are primarily the more complex nature of synthesizing said conjugates, but also the conjugates might not displace in the same manner due to a higher affinity and therefore would ead to a decrease in assay sensitivity.

The use of malachite green enabled the displacement assay to achieve a LoD within the $\mathrm{mM}$ range, which is ideal for solid sample analysis. Improvements 
to the sensitivity could be achieved by developing smaller particle sized MIPs, and therefore increasing the mass transfer between analyte and receptor. The increased interaction would yield higher sensitivity particles that would allow a lower LoD to be secured [12]. Having a lower LoD would facilitate the analysis of other samples and would expand the technology towards other compounds of interest that require more sensitive approaches [13]. Methods to be considered for the development of smaller more homogenous MIPs are the solid phase synthesis of the receptors, though this would require the chemistry behind the coupling of the template to a solid phase support to be investigated [14]. As a proof-of-concept the research highlights that it is possible to generate an easy low cost analytical assay for easily available commercial components, and therefore has merit.

Extending proof-of-concepts towards other analytical targets can be problematic, and concepts may only extend as far as the model compound in some situations. Therefore, the proof-of-concept was extended in chapter 5 tailoring the displacement assay towards the detection of a classical drug of abuse. The processes conducted reflected that of the first research article based on this technology, though this time with amphetamine hydrochloride as a template molecule. The concept was successfully implemented, demonstrating a good LoD (0.009 $\left.\mathrm{mg} \mathrm{mL}^{-1}\right)$ with the use of bulk imprinted MIPs and crystal violet dye as a reporting agent. The concept was taken one-step further, proving that the assay functioned when introduced to spiked urine samples and the resulting coloured filtrate could be easily observed due to the unnatural purple colour of the filtrate. The results reported show the same reproducibility in both urine and PBS, though for further biological sample analysis other biomolecules and ions must be taken into consideration. The assay must be tested against common biomolecules, ionic species, and medications that maybe found in biological samples before the assay can be truly announced a success in the analysis of a bio-relevant matrix [15].

In the previous two chapters illicit substances were selected as targets for the developed dye displacement assays, Chapter 6 therefore intended to demonstrate how the technology could be developed towards an environmental pollutant. Antibiotics were selected as such a pollutant, being a major risk to not only the environment but also the wellbeing of the human species as a whole [16]. Since their discovery in the $20^{\text {th }}$ century, antibiotics have seen a meteoric increase in use over the last decades, meaning their presence in the environment has seen the same increase. This makes them the 
perfect target for a MIP-based colorimetric sensor that would facilitate the rapid analysis of contaminated environmental and food samples.

As in Chapters 4 \& 5, the same methodology of developing a colorimetric assay was implemented, though this time a more elegant method of producing more homogenous MIP particles was selected. The use of an emulsion polymerization strategy was chosen, combining an aqueous phase and organic phase in the presence of a surfactant that facilitated the formation of a stable emulsion. The research draws attention to the amount of agitation (stirring) the components require to achieve defined particle sizes, and analyzes the distribution of said sizes in each sample. It was found as the amount of agitation increased the particle size decreased, though this began to plateau after $1000 \mathrm{rpm}$ with a particle size of $8-10 \mu \mathrm{m}$. If smaller particle sizes wanted to be achieved considerations towards the use of an ultrasonic probe could be made, with some examples in literature using said device to produce nanosized MIPs [17]. The downside to producing sub-micron particles would however mean that the methods used to collect and isolate said particles would become more difficult. Particles of a low micron size prove easier to handle and process, making them more "useful" in the currently developed dye displacement assay format.

The optimum MIP composition was found to be a methacrylic acid based TRIM co-polymer, with the acid functionalized MIPs out performing the basic functionalised MIPs. Analysis of the structure of the aminopenicillin used (amoxicillin) reveales the presence of both acidic and basic functionalities, though the basicic functionality is arguably less easily accessible. A composite material of both the acidic and basic monomer could theoretically yield improved results, though this may be unlikely as the opposing monomers are just as likely to interact with each other than with the templated amoxillicin during the MIP synthesis [18]. A promising if not surprising result is however the higher sensitivity of the emulsion MIPs in comparison to the monolithic bulk MIPs. The greater homogeneity in size and morphology is highlighted, offering an increased possibility of large scale production that could lead to easier implimention of MIPs into sensory platforms in both a research and commercial setting.

The developed assay format stemming from the development of the aforementioned MIP demonstrates once more that all MIPs have the potential to be easily converted into a colorimetric assay. As with the previous two 
assays, multiple dye molecules were tested for being displaced. Mordant orange was selected as the dye of choice, though unlike with the assays prior the dye molecules utilized this time were smaller and lower in molecular weight than the imprinted amoxicillin. The colorimetric assay was tested against amoxicillin, ampicillin, and cloxacillin and the assays response analyzed. The response towards amoxicillin was the strongest, with a visually apparent amount of dye being displaced and quantified with a UV-vis spectrophotometer. Cloxacillin and ampicillin showed a diminished response with the assay, though still a weak response was observed. Selectvity towards amoxicillin was favoured, though arguably it would be more benifical from a sensory and commercial standpoint if the assay was selective towards all aminopenicillins. Having a colorimetric sensor that could detect molecules across the class is more useful than solely targeting one antibiotic as many different molecules tends to be prescribed depending on the attached ailment [19]. It would therefore stand to reason if the MIP was redeveloped to be less selective towards the one aminopenicillin, as in this situation the ability to detect beyond the single compound while doing in-the-field analysis would be highy beneficial compared to single molecule analysis.

Overall the presented research across Chapters 4, 5, \& 6 draws clear attention to how MIPs can be easily implemented into colorimetric sesnors. In the future further research and development should target the development of more sensitive and homogenous MIPs, increasing the analytical capabilities of the sensors towards trace amount analysis and bringing the sensitivity closer to that of bio-based assays. Other considerations should be made towards the use of more sustainable dyes, with traditional dye molecules being generally toxic and harmful towards the environment [20]. Identification of bio-friendly alternatives would reduce the environmental impact, alongside enabling the potential for the development into more biocompatible scenarios e.g. wearable sensors [21]. 


\section{Chapter 7 References}

[1] Graziano, S.; Anzillotti, L.; Mannocchi, G.; Pichini, S.; Busardo, F. P. Screening methods for rapid determination of new psychoactive substances (NPS) in conventional and nonconventional biological matrices. J Pharm \& Biomed Anal. 2019, 163, 170-179.

[2] Smith, J. P.; Sutcliffe, O. B.; Banks, C. E. An overview of recent developments in the analytical detection of new psychoactive substances (NPS). Analyst. 2015, 140, 4932-4948.

[3] Al-Saffar, Y.; Stephanson, N. N.; Beck, O. Multicomponent LC-MS/MS screening method for detection of new psychoactive drugs, legal highs, in urine - experience from the sweidsh population. J Chroma B. 2013, 930, 112-120.

[4] Castaing-Cordier, T.; Ladroue, V.; Besacier, F.; Bulete, A.; Jacquemin, D.; Giraudeau, P.; Farjon, J. High-field and benchtop NMR spectroscopy for the characterization of new psychoactive substances. Forensic Sci Int. 2021, 321, 110718.

[5] Bade, r.; White, J. M.; Chen, J.; Baz-Lomba, J. A.; Been, F.; Bijlsma, L.; Burgard, D. A.; Castiglioni, S.; Salguerio-Gonzalez, N.; Celma, A.; Chappell, A.; Emke, E.; Steen, R.; Wang, D.; Zuccato, E.; Gerber, C. International snapshot of new psychoactive substance use: case study of eight countries over the 2019/2020 new year period. Water research. 2021, 193, 116891.

[6] Srivastava, J.; Kushwaha, A.; Srivastava, M.; Srivastava, A.; Singh, M. Glycoprotein imprinted RGO-strach nanocomposite modified EQCM sensor for sensitive and specific detection of transferrin. J Electro Anal Chem. 2019, 835, 167-177.

[7] Crapnell, R. D.; Jesadabundit, W.; Ferrari, A. G. M.; Dempsey-Hibbert, N. C.; Peeters, M.; Tridente, A.; Chailapakul, O.; Banks, C. E. Toward the rapid diagnosis of sepsis: detecting interleukin- 6 in blood plasma using functionalization screen-printed electrodes with thermal detection methodology. Anal. Chem. 2021, 93(14), 5931-5938.

[8] Habibzadeh, P.; Mofatteh, M.; Silawi, M.; Ghavami, S.; Faghihi, M. A. Molecular diagnostic assays for COVID-19: an overview. Critical reviews in clinical lab sci. 2020, 20, 1040-8363.

[9] Li, W.; Zhang, X.; Li, T.; Ji, Y.; Li, R. Molecularly imprinted polymer-enhanced biomimetic paper-based analytical devices: a review. Anal Chimi Acta. 2021, 1148, 238196. [10] Soovali, L.; Room, E.; Kutt, A.; Kaljurand, I.; Leito, I. Uncertainty sources in UV-vis spectrophotometric measurement. Accreditation and quality assurance. 2006, 11, 246-255.

[11] McNiven, S., Kato, M., Levi, R., Yano, K., \& Karube, I. Chloramphenicol sensor based on an in situ imprinted polymer. Analytica Chimica Acta. 365, 69-74 (1998).

[12] Wackerlig, J.; Schirhagl, R. Applications of molecularly imprinted polymer nanoparticles and their advances toward industrial use: a review. Anal Chem. 2016, 88(1), 250-261.

[13] Sharma, P. S.; Iskierko, Z.; Pietrzyk-Le, A.; D'Souza, F.; Kutner, W. Bioinspired intelligent molecularly imprinted polymers for chemosensing: a mini review. Electrochem Сотти. 2015, 50, 81-87.

[14] Poma, A.; Guerreiro, A.; Whitcombe, M. J.; Piletska, E. V.; Turner, A. P .F.; Piletsky, S. A. Solid phase synthesis of molecularly imprinted polymer nanoparticles with a reusable template - "plastic antibodies". Adv Func Mater. 2013, 23(22), 2821-2827. 
[15] Li, W.; Zhang, J.; Tse, F. L. S. Strategies in quantitative LC-MS/MS analysis of unstable small molecules in biological matrices. Biomed Chroma. 2011, 25, 258-277.

[16] Ding, C.; He, J. Effect of antibiotics in the environment on microbial populations. Appl MicroBio \& Bio Tech. 2010, 87, 925-941.

[17] Canselier, C. S.; Delmas, H.; Wilhelm, A. M.; Abismail, B. Ultrasound emulsification - An overview. J Dispersion Sci \& Tech. 2001, 23, 333-349.

[18] Zhang, Y.; Miyake, G. M.; John, M. G.; Falivene, L.; Caporaso, L.; Cavallo, L.; Chen, E. Y. X. Lewis pair polymerization by classical and frustrated lewis pairs: acid, based and monomer scope and polymerization mechanism. Dalton Trans. 2012, 41, 9119-9134.

[19] Zhang, X.; Guo, W.; Ngo, H. H.; Wen, H.; Li, N.; Wu, W. Performance evaluation of powdered activated carbon for removing 28 types of antibiotics from water. J Environ Manag. 2016, 172, 193-200.

[20] Khattab, T. A.; Abdelrahman, M. S.; Rehan, M. Textile dyeing industry: environmental impacts and remediation. Environ Sci Pollu Res. 2020, 27, 3803-3818.

[21] Jiang, X.; Chen, R.; Zhu, H. Recent progress in wearbale tactile sensors combined with algorithms based on machine learning and signal processing. APL Mater. 2021, 9(3), 030906. 
Appendices

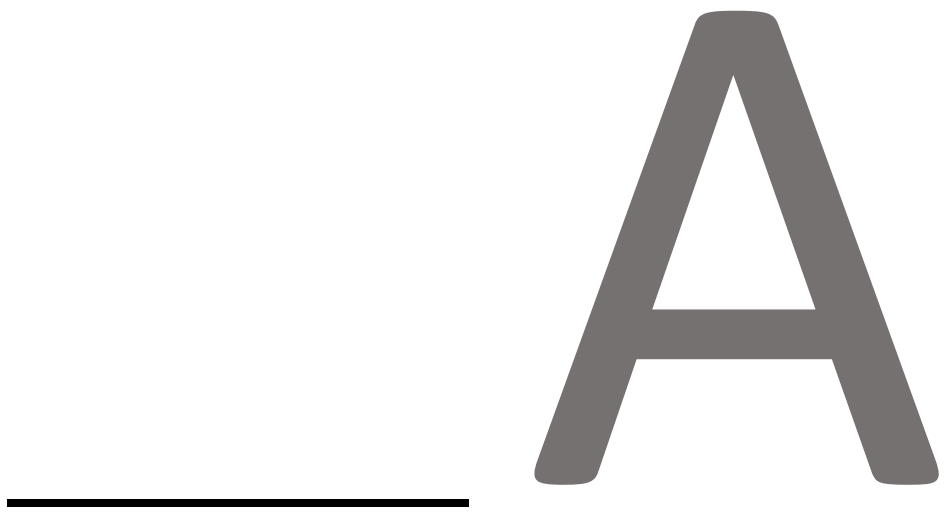




\section{Summary}

The presented thesis investigates the uses of MIPs in various sensing applications, drawing close attention to the possibility of using MIPs in lowcost sensors and assay formats. More specifically, sensing arrays were investigated that would prove beneficial to the in-the-field-testing of illicit or controlled substances.

Chapter 1 introduces the idea of molecular sensing and the different methods developed for the analysis of molecular species. This builds the bigger sensing picture, directing the reader towards the use of molecularly imprinted polymers in sensing platforms.

Chapter 2 conducts a deep dive into the current status quo of molecularly imprinted polymers, drawing specific attention towards low-cost sensing platforms and assay formats. The review paper shows how MIPs have been applied to the sensing to a whole range of molecular species on a research level, though struggle to be applied in a commercial setting. One of the reasons discussed hinges on the reliability and reproducibility of MIPs, which in turn also questions the associated sensing platforms that utilize these synthetic receptors.

Chapter 3 seeks to offer a solution to the reliability and reproducibility questions were raised in the previous chapter. To this end, a method of directly grafted molecularly imprinted polymeric receptor layers is highlighted. The methodology discussed enables the synthesis of receptor layers across the surface of an aluminium substrate, thus allowing the measurement of the targets presence via thermal impedance. The selected target for the experiments was the New Psychoactive Substance (NPS) 2-methoxphenidine, demonstrating that MIPs could be used to detect newly found substances of concern for law enforcement. The presented results demonstrate a superior limit of detection (LoD) of the sensor compared to traditionally generated receptor layers. Correlating the thickness of the newly grafted layers to the LoD achieved.

Chapter 4 developed the idea of sensing controlled substances further, by designing a sensing method that is not reliant on electrical transducers. The proposed dye displacement assay demonstrated how a MIP could be modified by preloading a dye that can be displaced to the surrounding medium in the presence of the target molecule. The resulting assay demonstrated a selectivity 
towards the NPS target, and showed a vibrant colour transformation that allowed for naked-eye detection of the chosen compound. The research builds a relationship between the imprint factor (IF) of a MIP and the binding capabilities (BF) of a dye molecule, aiming to generate a mathematical probability of the dye being displaced by the target substrate. Various other facets of the sensor are investigated, optimising the incubation time, and amount of MIP required to generate a colorimetric response.

Chapter 5 builds further on the concept of dye displacement assays, replicating the process for a more traditional drug of abuse (amphetamine). This work highlights how the concept can be adapted to other compounds and not just the model compound introduced in the previous paper, thus lending to its potential for commercial viability. Various dye molecules (crystal violet, basic blue, pararosaniline, methyl orange and phenol red) are tested for suitability as a displaceable dye indicator, with crystal violet proving the most suitable. The sensor is shows to displace dye in a quantitative manner, relating the amount of amphetamine present to the amount of dye displaced. The research culminates in the use of the sensor in spiked urine, providing similar amount of dye displaced in both urine and PBS.

Chapter 6 expands on the knowledge base previously built in the prior two chapters, and extends the use of dye displacement assays towards the detection of antibiotics. In this chapter, the rational design of a MIP towards the detection of amoxicillin is outlined, proposing various monomer: crosslinker pairings for the binding of the antibiotic in aqueous medium. These compositions were implemented in monolithic bulk imprinted polymers, with the best composition (MAA/EGDMA) being carried forwards to the development of MIP particles by an emulsion polymerization approach. The more elegantly synthetized particles showed greater homogeneity in both terms of size and morphology over their monolithic counterparts, leading to their use in the ensuing assay. Mordant orange was selected as the dye to be pre-loaded onto the MIP for the assay after a study with itself, malachite green and crystal violet. The produced sensor was then subjugated to amoxicillin, cloxacillin, and ampicillin, enabling the sensitivity and selectivity of the assay to be evaluated.

Finally, the results are critically analyzed in Chapter 7. In this chapter, the research findings are debated, drawing attention to the windfalls and pitfalls of each of the developed sensors. 


\section{Valorisation}

The transfer of knowledge from a research environment to the commercial stage is a critical process that is becoming more relevant than ever before. The development of new technologies that can benefit society is a continuous process, with hundreds of breakthroughs in many areas of science each year. Though this is true, the vast amount of these breakthroughs will not see the shelves in a store, as the translation from laboratory research to commercial application is expensive, time-consuming and sometimes goes completely unnoticed. It is therefore imperative for researchers to be aware of the true impact of their research, and to recognise when a technology is capable of making the leap to the commercial sector. Potential impact of research is therefore always at the forefront of a researchers mind, with institutions incentivising the transition of research into novel products and/or services.

The work in this thesis described the application of MIPs in multiple sensory platforms, with the research orientated towards investigating new ways of utilizing MIPs and sensing molecules.

The current landscape of MIPs integrated into sensory platforms was discussed in chapter $\mathbf{2}$, concluding that the current synthetic procedures and methods incorporating MIPs need to become more reliable/reproducible to yield products that are suitable in a commercial setting. This said, MIPs still have excellent potential for revolutionising the field of sensing, and offering affordable products for the analysis of a whole host of molecules.

MIPs' unique selling point lies in the simplicity of the technology, solely comprising of a polymeric matrix that has complimentary nanocavities tailored towards the binding of a target molecule. The ways in which this nanocavity is synthesized may vary, yet the premise remains the same across the field. This simplicity lends itself to the cheap production of generic MIPs, with the constituent elements of a MIP comprising from a functional monomer, functional crosslinker, porogen, and initiator molecule (though component ratios would still need tweaking based on the selected target). All of which are commercially available in large amounts at low cost, primarily due to the advancements made in the chemical industry and the developments in polymer science over the last decades.

The main restricting factor holding MIPs back from a commercial environment is the scaling of mass produced MIPs, where batch-to-batch variance is low and 
reproducibility is high. Steps have been made towards resolving this matter with companies such as MIP diagnostics Ltd. [1] pathing the way with their solid phase synthesis of nanoMIPs. Their development allows the synthesis of highly reproducible nanoparticle MIPs by generating polymeric receptors on a column loaded with template functionalized glass beads. A polymer solution is added down the column, followed by exposure to UV light, and finally the elution of unreacted components/low affinity MIPs. The high affinity MIPs are then washed off the column with warm water, resulting in highly dispersed nanoMIPs in solution [2]. These nanoMIPs have therefore been applied in many applications, including lateral flow assays, low-throughput ELISA, highthroughput ELISA and other sensory devices [3]. The solid phase synthesis of these nanoMIPs has been proven to be a game changer, however the limitations still exist with the current synthesis of these artisan MIPs being carried out on a small scale.

The future commercial success of MIP-based sensing technology does not only rest in the hands of solid phase synthesis alone, other improvements to the way MIPs are being implemented into sensor platforms may hold just as much value, if not more. In chapter 3, the use of MIPs in conjunction with the "Heat Transfer Method" (HTM) is presented, highlighting how the sensitivity of the platform could be improved by applying a new MIP deposition method. This technology is of high value, using a simple thermal resistor, a thermal couple, flow cell and power unit to measure the binding affinities of MIPs. The simplistic nature of the methodology is similar to other innovations in the field such as electrical impedance and quartz crystal microbalance (QCM), though easier and cheaper to create such a device in comparison. In theory, this is a technology that could be easily used in "the field", being easily transportable and compact in comparison to other current analytical methods that require large equipment for analysis. This reasoning is why the technology was patented by Maastricht Univeristy and other participating universities, seeing the huge research and commercial potential of the platform. Currently this technology still remains in the developmental stages of a company coined "Sensip"(Maastricht university based spin-off company), calibrating the platform towards the detection of bacteria using surface imprinted polymer opposed to molecularly imprinted polymers [4]. The hurdle remains the same with this platform, the production of the receptor layers.

A lot of products are commercial successes due to their ease of use and handsized portability, a facet MIP based readout methods has not leaned into as of 
yet. Chapters 4, 5, \& 6 investigated producing MIP-based dye displacement assays removing the need for a readout platform all together. This approach took simple bulk imprinted MIPs, commercially available dyes and combined the two to create the dye displacement assay. The straightforward manner in which this displayed would make for the easy production of colorimetric assays from any MIP. A proposal was therefore made to investigate the feasibility of taking the technology and developing a product from it. Funding was applied for from NWO, outlining a strategy for the production of MIPbased dye displacement assays for the rapid analysis of drug samples. The concept itself was highly promising, though again the reliability and reproducibility of MIPs was brought into question leading to funding be declined.

Overall, the field of MIPs is not ready to be brought to the commercial sensing sector. Key obstacles must be overcome to even consider transitioning a product from a research environment, though a lot of MIP based technologies are on the cusp of achieving this. As with more traditional biosensors before them, it is only a matter of time before MIP based technologies see the market. 


\section{References}

[1] MIP diagnostics, https://www.mip-dx.com/, (March $5^{\text {th }}$ 2021)

[2] Poma, A.; Guerreiro, A.; Whitcombe, M. J.; Piletska, E.; Turner, A. P. F. ; Piletsky, S. A. Solid-phase synthesis of molecularly imprinted polymer nanoparticles with reusable template - "plastic antibodies". Adv Funct Mater. 2013, 23(22), 2821-2827.

[3] Smolinksa-Kempisty, K.; Guerreiro, A.; Canfarotta, F.; Caceres, C.; Whitcombe, M. J.; Piletsky, S. A comparison of the performance of molecularly imprinted polymer nanoparticles for small molecule targets and antibodies in the ELISA format. Sci Reports. 2016, 6, 37638 .

[4] Sensip, https://www.sensipdx.com/, (March $5^{\text {th }}$ 2021) 


\section{Curriculum Vitae}

Joseph W. Lowdon MChem (Hons) obtained an integrated master's degree in chemistry from Manchester Metropolitan University in 2017. Over the course of his studies, he participated in multiple internships in the fields of synthesis and characterization of New Psychoactive Substances, synthesis of fluorescent monomers, and intelligent design of molecularly imprinted polymers. Since November 2017, he has begun to obtain his $\mathrm{PhD}$ in chemical and biological biosensing within the Sensor Engineering Group of the Faculty of Science and Engineering at Maastricht University. His research interests include synthesis of biomimetic polymers for bio- and chemical sensing, biocompatible materials for drug delivery and the implementation of new technologies for healthcare management. 


\section{Publications list}

Casadio, S.; Lowdon, J. W.; Betlem, K.; Ueta, J. T.; Foster, C. W.; Cleij, T. J.; van Grinsven, B.; Sutcliffe, O. B.; Banks, C. E.; Peeters, M. Development of a novel flexible polymer-based biosensor platform for the thermal detection of noradrenaline in aqueous solution, Chem Eng J. 2017, 315, 459-468.

Lowdon, J. W.; Alkirkit, S. M. O.; Mewis, R. E.; Fulton, D.; Banks, C. E.; Sutcliffe, O. B.; Peeters, M. Engineering molecularly imprinted polymers (MIPs) for the selective extraction and quantification of the novel psychoactive substance (NPS) methoxphenidine and its regioisomers. Analyst. 2018, 143, 2002-2007.

Eersels, K.; Diliën, H.; Lowdon, J. W.; Steen Redeker, E.; Rogosic, R.; Heidt, B.; Peeters, M.; Cornelis, P.; Lux, P.; Reutelingsperger, C. P.; Schurgers, L. J.; Cleij, T. J.; van Grinsven, B. A novel biomimetic tool for assessing vitamin K status based on molecularly imprinted polymers. Nutrients. 2018, 10, 751.

Lowdon, J. W.; Eersels, K.; Rogosic, R.; Boonen, T.; Heidt, B.; Dilien, H.; van Grinsven, B.; Cleij, T. J. Surface grafted molecularly imprinted polymeric receptor layers for thermal detection of the New Psychoactive Substance 2methoxphenidine. Sensors \& Actuators A: Physical. 2019, 295, 586-595.

Lowdon, J. W.; Eersels, K.; Rogosic, R.; Heidt, B.; Diliën, H.; Steen Redeker, E.; Peeters, M.; van Grinsven, B.; Cleij, T. J. Substrate displacement colorimetry for the detection of diarylethylamines. Sensors \& Actuators B: Chem. 2019, 282, 137144.

Rogosic, R.; Lowdon, J. W.; Heidt, B.; Diliën, H.; Eersels, K.; van Grinsven, B.; Cleij, T. J. Studying the effect of adhesive layer composition on MIP-based thermal biosensing. Physica Status Solidi (A). 2019, 216, 1800941.

Heidt, B.; Rogosic, R.; Lowdon, J. W.; Desmond-Kennedy, M.; Jurgaityte, K.; Orri, J. F.; Kronhorst, Y.; Mendez, S.; Polyakova, E.; Rice, H. T.; Crijns, F.; Dilien, H.; Steen Redeker, E.; Eersels, K.; van Grinsven, B.; Cleij, T. J. Biomimetic bacterial identification platform based on thermal transport analysis through surface imprinted polymers: From prrof of principle to proof of application. Physica Status Solidi (A). 2019, 216, 1800688.

Heidt, B.; Rogosic, R.; Bonni, S.; Passariello-Jansen, J.; Dimech, D.; Lowdon, J. W. ; Arreguin-Campos, R.; Steen Redeker, E.; Eersels, K.; Diliën, H.; van 
Grinsven, B.; Cleij, T. J. The Liberalization of microfluidics: Form 2 benchtop $3 \mathrm{D}$ printing as an affordable alternative to established manufacturing methods. Physica Status Solidi (A). 2020, 217, 1900935.

Lowdon, J. W.; Ishikura, H.; Radchenko, A.; Arreguin-Campos, R.; Rogosic, R.; Heidt, B.; Jimenez Monroy, K.; Peeters, M.; Diliën, H.; Eersels, K.; Cleij, T. J.; van Grinsven, B. Rapid colorimetric screening of elevated phosphate in urine: A charge transfer interaction. ACS Omega. 2020, 33(5), 21054-21066.

Lowdon, J. W.; Eersels, K.; Arreguin-Campos, R.; Caldara, M.; Heidt, B.; Rogosic, R.; Jimenez Monroy, K.; Cleij, T. J.; Diliën, H.; van Grinsven, B. A molecularly imprinted polymer-based dye displacement assay for the rapid visual detection of amphetamine in urine. Molecules. 2020, 25(22), 5222.

Lowdon, J. W.; Diliën, H.; Singla, P.; Peeters, M.; Cleij, T. J.; van Grinsven, B.; Eersels, K. MIPs for commercial application in low-cost sensors and assays An overview of the current status quo. Sensors $\mathcal{E}$ Actuators B: Chemical. 2020, $325,128973$.

Rogosic, R; Heidt, B.; Passariello-Jansen, J.; Bjornor, S.; Bonni, S.; Dimech, D.; Arreguin-Campos, R.; Lowdon, J. W.; Jimenez-Monroy, K. L.; Caldara, M.; Eersels, K.; van Grinsven, B.; Cleij, T. J.; Diliën, H. Modular science kit as a support platform for STEM learning in primary and secondary school. J. Chem. Education. 2021, 98(2), 439-444.

Lowdon, J. W.; Diliën, H.; van Grinsven, B.; Eersels, K.; Cleij, T. J. Colorimetric sensing of amoxicillin facilitated by molecularly imprinted polymers. Polymers. 2021, 13(13), 2221.

Arreguin-Campos, R.; Eersels, K.; Lowdon, J. W.; Rogosic, R.; Heidt, B.; Caldara, M.; Jimenez-Monroy, K. L.; Diliën, H.; Cleij, T. J.; van Grinsven, B. Biomimetic sensing of Escherichia Coli at the solid-liquid interface: From surface-imrpinted polymer synthesis towards real sample sensing in food safety. MicroChemical. J. 2021, 169, 106554.

Lowdon, J. W.; Ishikaru, H.; Kvernenes, M. K.; Cleij, T. J.; van Grinsven, B.; Eersels, K.; Diliën, H. Idenitfying potential machine learning algorithms for the simulation of binding affinities to molecularly imprinted polymers. Computation. 2021, 9(20), 103. 


\section{Acknowledgements}

Finally, I would like to acknowledge the people who have helped me throughout the process of conducting research, writing papers and writing my thesis. There has been numerous people who have helped me along the way; however, I wish to draw special attention to the people list below. Without their help, these last 4 years would have been a massive struggle. So this segment is dedicated to all those people who have listened to me moan, who have been there for the highlights, the lowlights, but most of all, who have supported me throughout my PhD journey.

Dr. Bart van Grinsven - Bart's passions lie in the collection of data, and then the subsequent publishing of papers from said data. This drive to conduct good science and push forward is something that makes Bart a great supervisor, making you strive to do research and publish. Since I have known Bart, he has built a sizable research group, growing year upon year. This charisma to build a team and to grow highlights what can be done with a little hard work. Working alongside Bart has therefore taught me many lessons about academia and what I have to do to succeed. As a supervisor, he has successfully navigated me through my time as a $\mathrm{PhD}$ and has prepared me for the next steps. Without Bart, I can safely say that none of this would have been possible, and for that, I would like to thank him.

Dr. Kasper Eersels - One of the most important aspects as a scientist is being and to see the scope and relevance of research. Dr. Eersels is a man who has no issue in seeing the bigger picture, easily pointing out the relevance of research and seeing how and where it fits in. This amazing capability comes from his wide spread knowledge across the scientific fields, being able to identify promising science and how it fits into the puzzle as a whole. Kasper therefore offers great advice and guidance, which has been essential for my success so far. He always has time to discuss ideas and orientate you in the correct direct, which is refreshing in the fast-paced world of research.

Prof. Dr. Thomas Cleij - If you ask anyone within the group what they think of when you mention Prof. Dr. Thomas Cleij, they would probably say Coca Cola. His fondness of the soft beverage has been the source of many hours of entertainment for the team, even leading to us wondering if he is actually sponsored by them. In all honesty, Prof. Dr. Cleij is a truly inspirational figure, being kind, supportive and a true academic. He is someone you rarely see, though still feel the presence of through his actions. I would therefore like to thank him for overseeing my $\mathrm{PhD}$, giving advice when required, and being the calmest person I may have ever met. 
Dr. Hanne Diliën - Being the chemist of the group, Dr. Diliën has always provided excellent advice regarding chemical processes and synthesis. Her knowledge is second to none, with her capacity to develop novel routes and molecules being extremely impressive. Her charismatic nature and passion for chemistry is inspirational and has enabled me to become a better chemist just through knowing her. Therefore, I would like to thank Hanne for not only making me a better chemist but for also giving me guidance throughout my research.

Debby Hewitt - Being organised is not something that comes naturally to me, luckily the team and I have Debby to always rely on. In truth, Debby is probably the sole reason that I find myself in the right place at the right time. Without her impressive organisational skills, willingness to communicate with others, and caring personality, the last four years would have been a lot tougher. She ensures that all the wheels keep turning behind the scenes, being a vital part of the team that we all admire and respect. I would therefore like to thank Debby for being a truly wonderful person, and for providing me with the help I needed.

Dr. Marloes Peeters - Prior to me coming to The Netherlands, Dr. Peeters supervised me in my undergraduate studies. Without her passion for MIPs (and sensors), I would have not found the field that I currently operate in. Due to her, I met my supervisors at a conference she was hosting and was offered my $\mathrm{PhD}$ position. Therefore I am extremely grateful for the opportunities that have been given to me as a direct result of us working together. Even now, we continue to have input on each other's research, and I hope this working relationship extends into the future.

Renato Rogosic - Renato was my very first colleague during my PhD experience, and over the last four years, we have become good friends. Since the very start, he has always been cool, reserved and level-headed, bringing a sense of calm to the lab. He is a brilliant engineer who has helped me both inside and outside of work. I would like to think his calm nature has rubbed off on me, but I fear my sarcastic British nature has instead eroded his Italian charm.

Ben Heidt - The passion Ben has for science and teaching astounds me every day. Ben lives and breathes science, being astonished by developments in all areas and constantly discusses bleeding edge topics. This enthusiasm is what drives Ben and has led to him produce some inspirational work. Alongside his keen scientific mind, Ben is also an extremely caring person who would go out of his way to help anyone. His point of view and support have therefore helped 
me immensely throughout my $\mathrm{PhD}$, offering unique insights and emotional support.

Rocio Arreguin-Campos - Working with Ro is a true pleasure, she is a kind and warm-hearted person who cares deeply for others. Researching topics with Ro and working alongside her is a great experience, with her being extremely pragmatic and attentive to detail. You can always rely on her for her honest opinion and trust her with any issues you may be having. She brightens up the lab and makes everyone feel welcome, being a key member of the team I think we would all agree we couldn't do without.

Manlio Caldara - Manlio has the great characteristic of being extremely optimistic and loves everything (everyone). These wonderful features of his personality make him a pleasure to work with and be around. The happiness he exudes are a catalyst for good, making everyone's day a little brighter than it would have been otherwise. I would therefore like to thank Manlio for making the lab and office a little more light hearted, and bringing a smile to everyone's faces.

Ramiro Marroquin-Garcia - Since joining the group, Ramiro has helped me inside and outside of the lab. He is an exceptional chemist with a fierce intellect that has in turn helped me develop as one. Alongside his vast polymer knowledge, Ramiro is a genuinely nice guy who would go out of his way to help anyone.

Kathia Jimenez-Monroy - I mainly worked with Kathia in a teaching capacity, learning to instruct students in chemical synthesis. Kathia is a kind and patient person who wants the best for the students under her care. Working with her taught me a lot, but mainly to trust in myself and be confident in a teaching scenario.

Martien Würdemann - Having an outsiders perspective on research and analysis is sometimes critical in order to progress. Martien is a person with great depth and knowledge of organic synthesis and analysis. His aid in using equipment and devising new synthetic pathways has been of great help throughout my time at Maastricht University. His immense knowledge and confidence has made me strive to be a better chemist, and I would like to thank him for pushing me in the right direction when sometimes I was a little off pace.

Technical staff at Maastricht Science Programme - As always the technical staff keeping the machines running and the lights on behind the scenes have 
my thanks. Without these people running the labs and ensuring everything is going smoothly, it would be impossible to carry out any form of research.

Ana Over - Out of everyone mentioned Ana is the person who has listened to me moan the most. Every day she has heard me moan about something related to my work, and for that, I am extremely grateful. Without her, I would have found the experience a hell of a lot harder than it would have been. She is my emotional rock, the person who believes in me the most, and is irreplaceable to me.

Anthony \& Kathrine Lowdon - My parent's unwavering support has been critical in the last few years. The emotional support they have provided throughout this process has kept me calm and pushing forward. I would therefore like to thank them not only for being my parents, but also for being a strong driving force behind me completing this process.

Yvo Over \& Sonja Arler - Alongside my own parents and my girlfriend, Yvo and Sonja are the people who have cared for me over the course of my PhD. Taking a keen interest in what I do and ensuring I remember to take time off, they have helped me understand Dutch culture both through language and food. 